\title{
RELATIVES' EXPERIENCES OF CRITICAL CARE
}

\author{
By \\ Felicity Hughes \\ Submitted for the degree of $\mathrm{PhD}$
}

European Institute of Health and Medical Sciences

University of Surrey

June 2005

(C) Felicity Hughes 2005 
ProQuest Number: 27605295

All rights reserved

INFORMATION TO ALL USERS

The quality of this reproduction is dependent upon the quality of the copy submitted.

In the unlikely event that the author did not send a complete manuscript and there are missing pages, these will be noted. Also, if material had to be removed, a note will indicate the deletion.

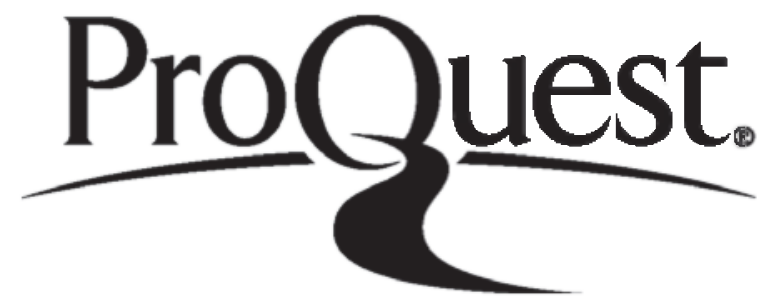

ProQuest 27605295

Published by ProQuest LLC (2019). Copyright of the Dissertation is held by the Author.

All rights reserved.

This work is protected against unauthorized copying under Title 17, United States Code Microform Edition (C) ProQuest LLC.

ProQuest LLC.

789 East Eisenhower Parkway

P.O. Box 1346

Ann Arbor, Ml 48106 - 1346 


\begin{abstract}
Aim

The aim of this research was to investigate relatives' experiences of critical care and staff perceptions of those experiences.

\section{Methods}

The study examined whether relatives found their experiences of critical care psychologically distressing, and explored the nature of communication processes between relatives and staff. It used a combination of qualitative (Phases I and III) and quantitative (Phase II) methods. All phases were conducted in the UK involving relatives of patients admitted to critical care units and staff working on those units. Phase I used semi structured interviews that were analysed according to grounded theory methodology. Phase II was a nationwide survey of information available to relatives within critical care and policies available to staff concerning care of relatives. Phase III used semi structured interviews and focus groups, which were analysed in accordance with grounded theory.

\section{Results and Conclusions}

The qualitative findings revealed that, overall, relatives were satisfied with their experiences and staff accurately perceived many aspects of these experiences. Relatives wanted to be kept informed of all stages of their patient's condition and received a mixture of verbal, factual information and reassurance which they found helpful. It is important that these aspects are not lost amid further improvements and developments. Many relatives suffer from a variety of stress symptoms; the enduring nature of these symptoms is unclear.

The process of communication and information giving between staff and relatives 
appears inconsistent and this was further illustrated by the quantitative findings in Phase II that demonstrated extensive variation of written information available to relatives and a lack of guidelines available to staff. Relatives report irregular contact with doctors and clear guidelines need to be established as to how often relatives should expect this contact. This would aim to allay expectations, largely created by the media, about the desirability of the presence of doctors and would reduce nurses' feelings of responsibility for the actions of doctors. In addition, relatives reported that they were content to receive the recommended written information examined in Phase II in a verbal format. Cost effective methods of relaying general information to relatives should be considered, for example, notice boards in waiting areas as opposed to information leaflets.

Nurses identified a gap in their training. Further training in communication, conveying difficult information and dealing with the responsibilities of relatives' complex emotional processes could be offered, targeted specifically towards nursing in critical care. There may be a need for employing an additional member of staff, for example, a Health Care Assistant with specific training to deal with advice and welfare issues related to critical care. This may help to address the skills overlap that occurs in critical care due to the nature of the tasks that nurses undertake.

Involving relatives in basic patient care, where appropriate, could have beneficial effects for patients, relatives and nurses, particularly in longer stay patients. It could aid relatives coping abilities by making them feel they have a purpose and may also free up nurses time to perform more complex tasks.

This research adds a qualitative perspective to existing quantitative literature. It updates previous qualitative studies that have become dated in the fast changing NHS and explores national information provision to relatives, something not attempted previously. 


\section{TABLE OF CONTENTS}

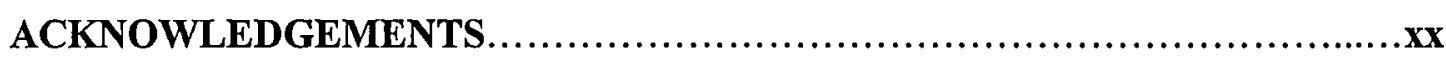

Chapter One: INTRODUCTION

1.1 The Development of Critical Care.............................................2

1.2 Definition of Critical Care.........................................................

1.3 Background Statistics......................................................

1.4 Main Government influences in recent years.................................8

1.4.1 Critical to Success: The place of efficient and effective critical care services within the acute hospital. Audit Commission (1999) 8

1.4.2 Comprehensive Critical Care: $A$ review of adult critical care services.

Department of Health (2000) 9

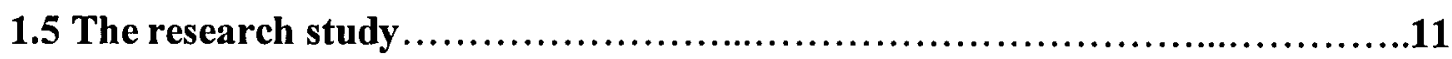

$\begin{array}{ll}\text { 1.5.1 Rationale } & 11\end{array}$

$\begin{array}{ll}\text { 1.5.2 Research questions } & 13\end{array}$

1.5.3 Definition of Relative 14

1.5.4 The Research Process 14

1.5.5 Implications of the study 
Chapter Two: REVIEW OF THE LITERATURE

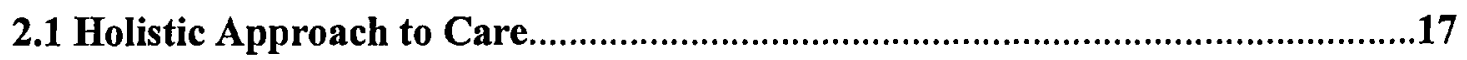

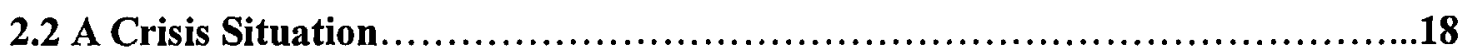

2.3 Intensive Care Unit Syndrome and Post Traumatic Stress....................21

2.4 Models of Coping .........................................................24

2.4.1 Direct Effect model vs. Buffering model 26

2.4.2 Emotion Focused- vs. Problem Focused Coping 30

2.4.3 Coping and Critical Care 33

2.4.4 The Concept of Hope 34

2.5 Environmental Factors.......................................................

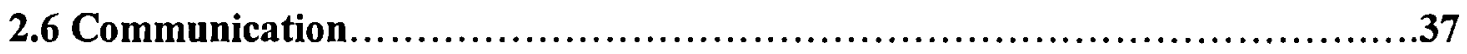

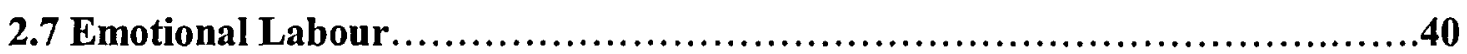

2.8 Relatives' Needs and Experiences.........................................43

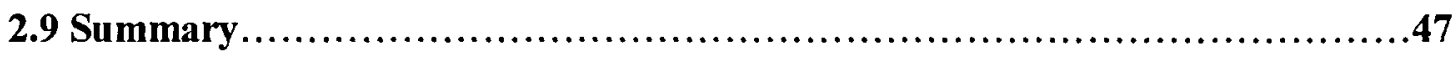


Chapter Three: RESEARCH PARADIGMS, METHODOLOGIES AND METHODS

3.1 Introduction. .49

3.2 Research Paradigms .49

3.3 Qualitative Research. .52

3.4 Evaluation of Qualitative Research. . .54

3.5 Qualitative Methodologies. .56

3.5.1 Grounded theory

3.5.2 Data Analysis

60

3.6 Qualitative Methods .61

3.6.1 Research Interviews 61

3.6.2 Focus groups 64

3.7 Quantitative Research. .65

3.8 Evaluation of Quantitative Research. .66

3.9 Quantitative Methods. .67 3.9.1 Surveys 67

3.10 Ethical Consideration. .69 
3.11 Summary . .73

Chapter Four: PHASE I: RELATIVES' EXPERIENCES AND STAFF

PERCEPTION OF RELATIVES' EXPERIENCES OF CRITICAL CARE

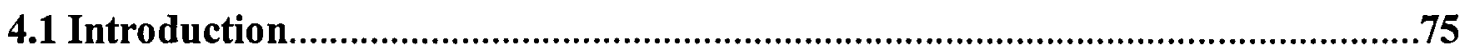

4.2 Objectives of Phase I........................................................

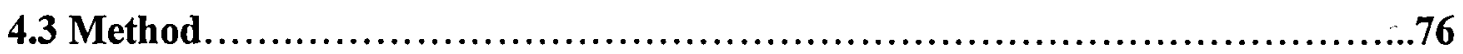

4.4 Recruitment, data collection and analysis.................................77

$\begin{array}{ll}\text { 4.4.1 Relatives recruitment } & 77\end{array}$

$\begin{array}{ll}\text { 4.4.2 Staff recruitment } & 78\end{array}$

$\begin{array}{ll}\text { 4.4.3 Data collection } & 78\end{array}$

4.4.4 Data Analysis

4.5 Ethical Considerations and Gate-Keeping Issues..............................79

4.6 Part A: Results and Discussion from Relatives Interviews...................81

4.6.1 Description of sample...................................................

$\begin{array}{ll}\text { 4.6.2 Themes } & 82\end{array}$

$\begin{array}{ll}\text { Relatives } & 84\end{array}$

Knowledge and Experience $\quad 84$

Emotional Reactions $\quad 86$

$\begin{array}{ll}\text { Learning } & 88\end{array}$ 
Torn Priorities 91

Patient Care $\quad 92$

Involvement in Patient Care 93

Care perceived as Inappropriate 93

Changes in patient behaviour $\quad 95$

$\begin{array}{ll}\text { Recovery } & 97\end{array}$

$\begin{array}{ll}\text { Information } & 97\end{array}$

Type of information needed 98

Type of Information given $\quad 99$

$\begin{array}{ll}\text { Unit Procedures } & 102\end{array}$

$\begin{array}{ll}\text { Established Procedures } & 102\end{array}$

Improvements 103

4.7 Part B: Results and Discussion from Staff Interviews......................105

$\begin{array}{ll}\text { 4.7.1 Description of sample } & 105\end{array}$

$\begin{array}{ll}\text { 4.7.2 Themes } & 105\end{array}$

$\begin{array}{ll}\text { Relatives } & 108\end{array}$

Knowledge and Experience 108

Emotional Reactions 109

$\begin{array}{ll}\text { Learning } & 110\end{array}$

Coping and Support $\quad 111$ 
Looking after the Relatives

Facilitating their Role

Perceptions

Information

Type of Information Needed

Type of Information Given

Importance of information

\section{Unit Procedures}

Established Procedures

System Problems

Improvements

Patient Care

Involvement in Patient Care 
Chapter Five: PHASE II: AN ANALYSIS OF INFORMATION AVAILABLE TO RELATIVES AND POLICIES CONCERNING RELATIVES IN CRITICAL

CARE

5.1 Introduction

5.2 Objectives of Phase II .134

5.3 Methods. .135

5.4 Results. .136

5.4.1 Description of sample. .136

5.4.2 Data Analysis

5.4.3 Information leaflets

5.4.4 Readability of Information leaflets

5.4.5 Content of policy documents

5.5 Discussion and Conclusion. .144 
Chapter Six: PHASE III: RELATIVES INFORMATION NEEDS IN CRITICAL

CARE

6.1 Introduction. .149

6.2 Objectives of Phase III.

6.3 Methodology and methods .150

6.4 Recruitment, data collection and data analysis. 150

6.4.1 Relative recruitment 150

6.4.2 Staff Recruitment 153

6.4.3 Data Collection 155

6.4.4 Data Analysis 156

6.5 Part A: Results and Discussion from Relatives' Interviews 156 6.5.1 Description of sample 156 6.5.2 Themes 156

Information Giving 160

Type of information 160

Modes of delivery 161

Quality of information 162

Quantity of information 163 
Information need

Frequency

Comprehension

Location for receiving information

Information givers

Reactions to the patient

Touching their patient

Seeing their patient

Talking to their patient

Feeling their patient is cared for

Wanting to be with their patient

Making own judgements about their patient

Perceptions of staff

Positive Perceptions of nursing staff

Negative perceptions of nursing staff

Staff concern for well being of relatives

Individuals

Good visitor role

Amount of contact with doctors

Questioning staff 
Machines

Atmosphere

Different to what expected

Walking through the unit

Reactions to the situation

Emotions

Coping

Effect on other family members

Perceived social support

Stress symptoms

Benefit of experience

Practicalities and procedures

Internal practicalities

Familiar faces

6.6 Part B: Results and Discussion from Staff Focus Groups 
Information giving

Information leaflets 202

Practical information 203

Specific information 204

Nursing Staff 205

Staff tasks 205

Difficulties 206

Looking after relatives 207

Putting the patient first 210

Interpreting relatives reactions 212

Time given to relatives 213

Emotional support for relatives 215

Doctors

Nurses perception of doctors ' responsibility

Doctor-relative contact

Feeling responsible for the actions of doctors

Relatives

Relatives' rights

Respect for staff 
Qualities of relatives

Involvement in patient care

The environment

The critical care unit

Relatives perceptions of the NHS

Visiting

Potential Changes

Communication

Training

Member of staff for relatives

The Staff Team

Continuity of care 240

Staff cohesion

Job satisfaction

Consistency within staff

6.7 Mismatches between relatives' and staff perceptions. 


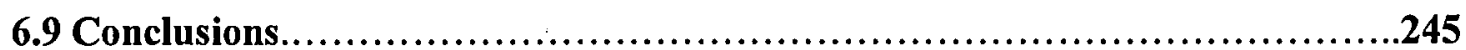

Chapter Seven: CROSS STUDY DISCUSSION AND CONCLUSION

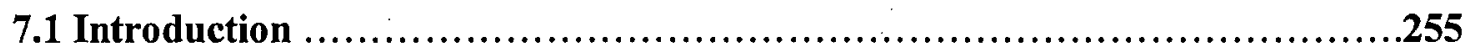

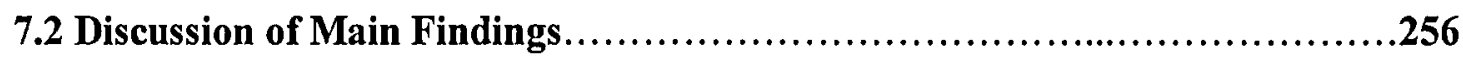

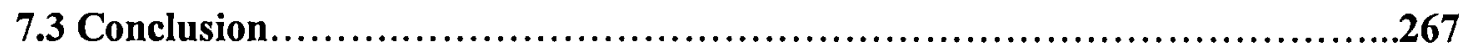

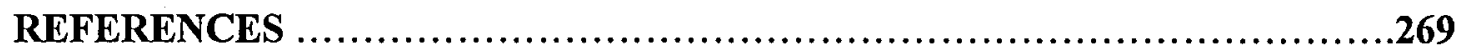

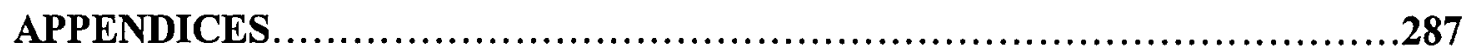




\section{TABLES}

\section{Chapter 1: Introduction}

Table 1 Guide to Comprehensive Critical Care...................................6

Chapter 3: Research Paradigms, methodologies and methods

Table 2 Summary of different processes of triangulation. .55

Chapter 4: Phase I Relatives' experiences and staff perceptions of relatives' experiences of critical care

Table 3 Range of emotional reactions relatives experience in critical care .86

Chapter 5: Phase II An analysis of information available to relatives and policies concerning relatives in critical care

Table $4 \quad$ Summary of the type of information contained in the leaflets for relatives 138

Table 5 Summary of the type of information included in policy document........140

Table 6. Summary of additional information available for relatives 143

Chapter 6: Phase III Relatives information needs in critical care

Table 7 Participant data for relatives

Table $8 \quad$ Participant data for staff from the neurological unit

Table 9 Participant data for staff from the general unit

Table 10 The range of relatives' emotions while a family member is in critical care 


\section{FIGURES}

\section{Chapter 1: Introduction}

Figure 1 Timeline of events that led to the development of critical care units.........3

Figure 2 Source of patients admitted to the average general critical care unit.....7

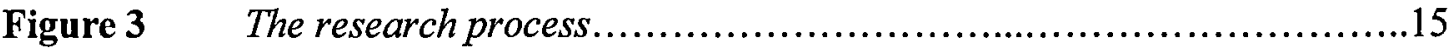

Chapter 2: Review of the literature

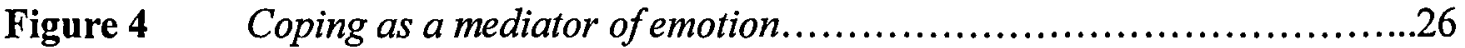

Figure $5 \quad$ Possible stress buffering mechanisms of social support.................29

Figure 6 Graphs of direct effect model of social support on symptomatology and two variations of the buffering model of social support on symptomatology

Chapter 3: Research Paradigms, methodologies and methods

Figure $7 \quad$ The research plan....................................................

Chapter 4: Phase I Relatives' experiences and staff perceptions of relatives' experiences of critical care

Figure 8 Themes that developed from relatives interviews.

Figure 9 Themes that developed from staff interviews

Chapter 5: Phase II An analysis of information available to relatives and policies concerning relatives in critical care 
Figure 10 Geographical location of responses.

\section{Chapter 6: Phase III Relatives information needs in critical care}

Figure 11 Themes that developed from interviews with relatives with a family member in critical care...........................................158

Figure 12 The process that relatives experience in critical care...................159

Figure 13 Themes that developed from focus groups with nursing staff...........200

Figure 14 Relatives interactions within critical care............................247

Figure 15 The process of giving information to relatives in critical care...........248

Figure 16 Factors influencing relatives coping methods while in a critical care

environment.....................................................252 


\section{APPENDICES}

Appendix A Letters of Ethical Approval..........................................289

Appendix B An example of an application to an Ethics Committee................297

Appendix C Phase I information sheet and consent form for relatives.................333

Appendix D Phase I information sheet and consent form for staff.................337

Appendix E Initial interview schedules for Phase I.................................

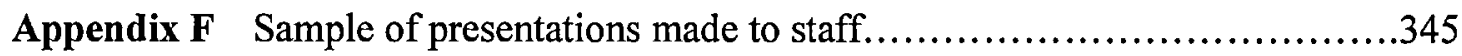

Appendix G Letter sent to the critical care units in Phase II...........................354

Appendix H Phase III information sheet and consent form for relatives...............357

Appendix I Phase III information sheet and consent form for staff..................361

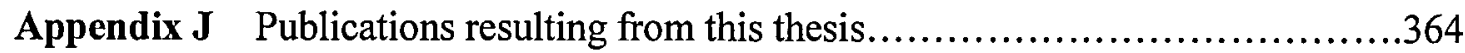




\section{Acknowledgements}

I would like to acknowledge and thank my supervisors Professor Karen Bryan and Professor Ian Robbins for their guidance and faith in my abilities throughout this $\mathrm{PhD}$. I would also like to thank the University of Surrey for giving me this opportunity by funding this $\mathrm{PhD}$.

I wish to thank all the hospitals that took part in every stage of this study, in particular, St George's Hospital, London. The staff at the critical care units there do an incredible job and gave me their full support to make this project work. This study could not have happened without the participation from relatives of critically ill patients; my sincere appreciation to all those who gave up their time to talk to me under such distressing circumstances.

My gratitude also to Mrs Enid Tubbs for answering my many questions with such patience and Dr Michele Peters for maintaining my sanity.

On a more personal note, I would like to thank Mr Ieuan Weir, for always believing in me. Finally, I would like to thank my mother, Mrs Deirdre Hughes, for your unfailing love, support and encouragement. Your strength is an inspiration to me.

This thesis is dedicated to my father, Mr Harvey Hughes (1948-1999), who told me as a little girl that I can do anything I put my mind to. 
Chapter One

\section{INTRODUCTION}




\subsection{The Development of Critical Care}

Critical care medicine is concerned with the management of patients who have acute, life threatening conditions within a specialised environment (Hinds \& Watson 1996). Specialized critical care units, in their most primitive form, did not exist until the 1950s. There were many different factors that contributed to this development. The major ones will be discussed below. Figure 1 illustrates some of the events that have led to the development of critical care units.

War has been a significant factor throughout history that has led to many kinds of medical advancements. Examples of which are transplant surgery during both World Wars (1914-1918 and 1939-1945 respectively) and, even earlier, Florence Nightingale's control of infection during the Crimean War (1854-1856). Nightingale reduced the death rate among wounded or sick soldiers from $40 \%$ to $2 \%$ (Porter 1997), mainly due to improved hygiene. She also created a high profile for nursing as respected profession, one which is crucial to today's health care system.

The National Health Service (NHS) was launched on the $5^{\text {th }}$ July 1948 . Its aim was to provide healthcare for all citizens, based on need rather than the ability to pay. The NHS brought a range of existing services under one organisation for the first time. Despite initial teething problems, mainly linked to administration and cost, by the 1960 s treatment was improving as better drugs were introduced.

Critical care units have become possible since the advent of artificial ventilation, used for the treatment of patients with respiratory failure, during the poliomyelitis epidemic in Copenhagen in the early 1950s. During this epidemic there was a shortage of 


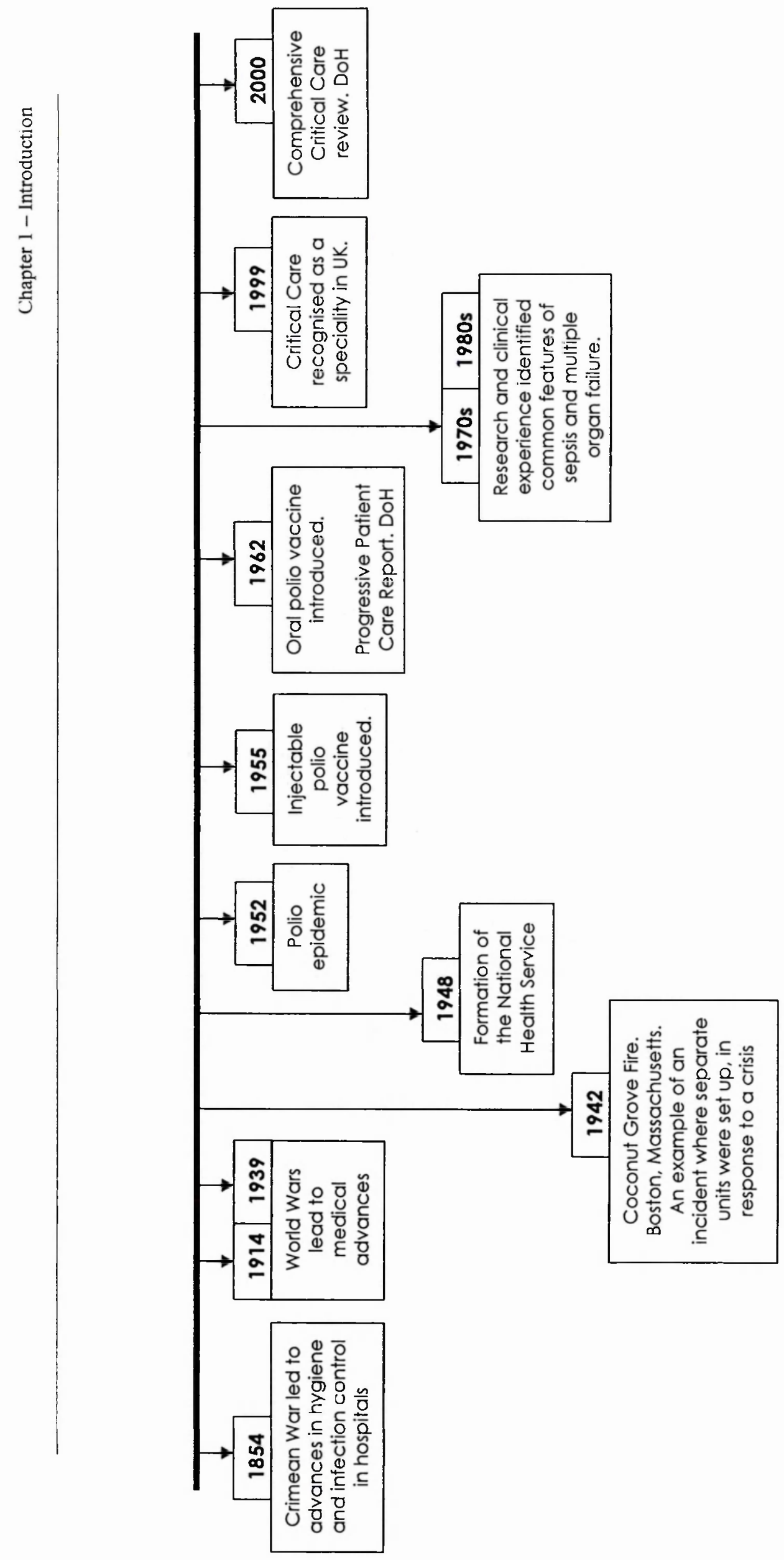

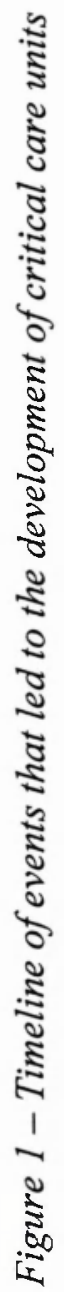


'iron lungs'. Doctors transferred a technique, only used in operating theatres, to pump air directly into the lungs. This technique was the foundation of today's ventilators and it almost halved the death rate at that time. During this time, vaccinations were being developed and in 1962 the oral polio vaccination was launched.

Since then, extensive advances in critical care medicine have been made and the field is not limited to artificial respiration. During the 1960 s continual monitoring of the major body systems, such as, renal, cardiovascular and respiratory systems, had become commonplace in the new critical care units. The computer became a vital tool in this procedure. Data was produced from the monitors at a rapid speed and it was only by automating tracking of this data that enabled it to be used in detecting crises, measuring therapeutic progress and watching trends to aid prognosis (Reiser 1993).

By 1970 s critical care units were widely available and drugs continued to advance. Increasingly complex interventions have been made possible since the ability to take over the role of a failing organ, allowing it time to recover. The 'Progressive Patient Care' report of 1962 (cited in Intensive Care Society 2003) outlined the "systematic grouping of patients according to their illness and dependence on the nurse, rather than by classification of disease or sex." The purpose built units mean staff can tend more readily to the needs of the critically ill and detect and respond to complications more quickly if, and when, they arise. Ultimately, the centralised unit improves patient outcome and reduces overall hospital costs (Hinds \& Watson 1996). In 1970 the Intensive Care Society was formed to bring together clinicians whose main interest was caring for critically ill patients. Critical care was only recognised as a speciality and awarded this status in the UK in 1999 (Intensive Care Society 2003). The main role of the society is education and 
research and it promotes a multidisciplinary approach to critical care to try to improve the profile of critical care with patients, other hospital services and the NHS in general (www.ics.ac.uk/home_menu/ics_about.htm).

\subsection{Definition of Critical Care}

The Intensive Care Society defines critical care, in the UK, as 'a service for patients with potentially recoverable conditions who can benefit from more detailed observation and treatment than is generally available in the standard wards and departments. Intensive care or intensive therapy should be differentiated from high dependency care although there is some overlap' (Intensive Care Society 1990). The Critical Care Unit, Intensive Care unit (ICU) or Intensive Therapy unit (ITU) is a specially staffed and equipped hospital ward committed to the care of patients with life threatening illnesses, injuries or complications (Oh 1997). Patients admitted to a critical care unit are classed as 'critically ill'. The unit provides the highest concentration of care in a hospital.

In 2001, new guidelines were introduced by the Department of Health classifying all patients into one of four groups based on need rather than illness, outlined in Table 1. Patients requiring critical care, in most cases, will have failure of the respiratory system, or have two other organ failures, if not related to the respiratory system. 
Table I-Guide to Comprehensive Critical Care (Department of Health 2000a).

\begin{tabular}{|ll|}
\hline Level of Care & Description of Condition \\
\hline 0 & $\begin{array}{l}\text { Treated on the general ward. } \\
\text { Require slightly more care than that received on the general } \\
\text { ward. } \\
\text { One organ failure, other than the respiratory system; classified } \\
\text { as high dependency } \\
\text { Either one organ failure, if it is part of the respiratory system, or } \\
\text { more than one organ failure if the organs are unrelated to the } \\
\text { respiratory system; classified as intensive care. }\end{array}$ \\
\hline
\end{tabular}

Throughout the literature 'intensive care' and 'critical care' have been referred to interchangeably. 'Critical Care' is the most recent terminology and is what will be used throughout this thesis, except if quotations state otherwise.

\subsection{Background Statistics}

A critical care unit has the highest staffing volume in a hospital with a 1:1 nurse:patient ratio, in contrast to a 1:12-15 nurse:patient ratio on a general ward and a 1:2 nurse:patient ratio on a High dependency unit (HDU). In order for patients to be moved out of a critical care unit, they have to be stable and capable of signalling for help.

Admissions to critical care come from a number of different sources, such as transfer from other hospitals or theatre. This is illustrated in Figure 2. Statistics for admissions to a general critical care unit from theatre range from $10-72 \%$ depending on whether major surgery is carried out at the hospital. Similarly, admissions from Accident and Emergency (A\&E) range from 3-44\% depending on the location of the hospital. The 
death rate on an average critical care unit is 1 in 5. Death rates are lower in combined ICU/HDU, but this still exceeds most other units in the hospital.

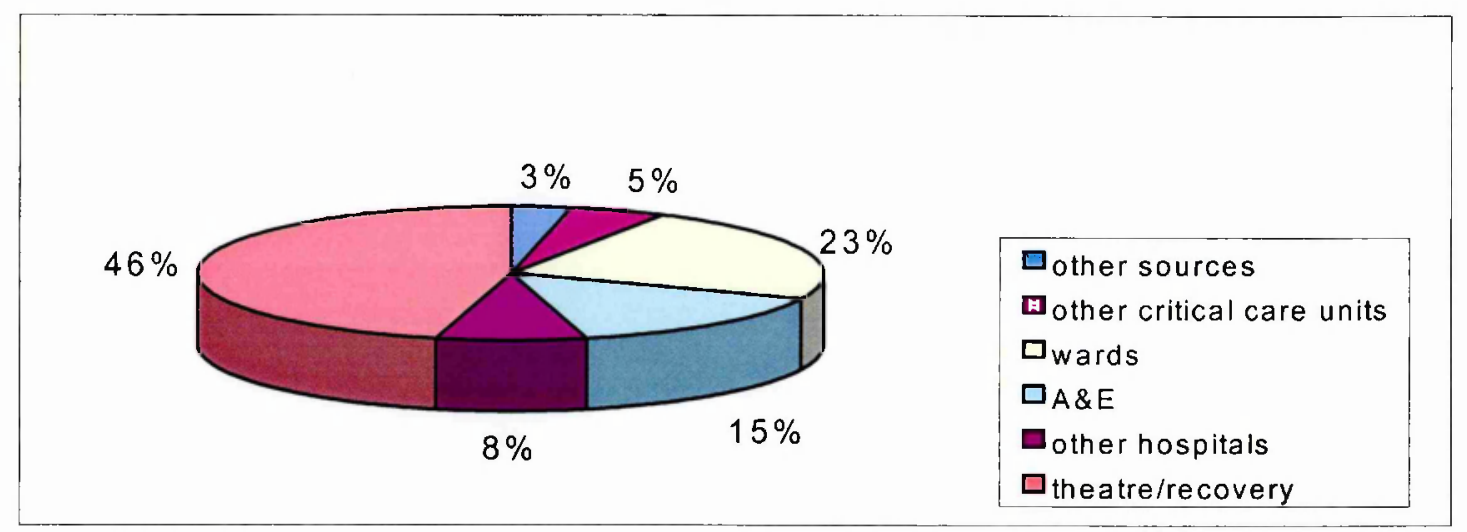

Figure 2 - Source of patients admitted to the average general critical care unit (Audit Commission 1999)

Due to the high staffing ratio and the intensity of highly technological equipment, critical care units are approximately 3-4 times more expensive to run that a general ward (Hinds \& Watson 1996) and absorb 15-20\% of hospital budgets (Wild \& Narath 2004). Compared with the rest of Western Europe the UK allocates a low proportion of beds to critical care. The number of acute beds allocated to general and specialist critical care in the UK is $2.7 \%$ ( 8.6 beds per 100000 population). Demark allocates the highest proportion in Europe with 4.6\%, followed by Austria with 3.8\%. In Europe as a whole, $18 \%$ of units have fewer than 6 beds. In the UK this figure is $48 \%$ (Department of Health 2000a). The only country in Europe with a lower proportion of critical care beds is Italy with $1.2 \%$, which still equates to 9.4 beds per 100,000 population. In comparison the USA allocates $6.3 \%$ of hospital beds to critical care services (Wild \& Narath 2004). In addition, patients in countries with a high density of critical care beds, for example Austria, demonstrate lower illness severity scores on standardised scales (e.g. APACHE, 
SAPS), than patients in countries with a lower density of beds, for example UK (Wild \& Narath 2004). Demand for critical care services continues to increase and complaints about lack of critical care beds continue to arise.

\subsection{Main Government influences in recent years}

In recent years many aspects of the NHS have undergone significant reform in order to provide for the changing health needs of the population throughout their lives. Two government documents that highlight potential change to critical care services are discussed below. These documents are referred to repeatedly throughout this thesis because of their specific implications for relatives and friends of critically ill patients.

\subsubsection{Critical to Success: The place of efficient and effective critical care services within the acute hospital. Audit Commission (1999)}

This report was undertaken because the Audit Commission deemed that critical care services (including critical care units, HDUs and specialist areas such as renal or coronary care units) are fragmented, expensive and under pressure. There are increasing costs of demand for beds continually exceeding supply and there is an acknowledgement that critical care services vary greatly between trusts. The report stated that there was an absence of useful management information about critical care resources, the treatments given and their effectiveness. It also makes explicit the link between organisation, efficiency and quality. The aim of this report was to try to help individual trusts improve their services. 
Recommendations made by this report were ranked as high, medium or basic priorities. One high priority recommendation was to find out relatives' views about their experiences in the unit via systematic satisfaction surveys. Further basic recommendations included reinforcing verbal information with plain language booklets for patients and relatives that explain:

$>$ The way the unit operates, location of facilities and contact names; what to expect on arrival in the unit (e.g. for major elective surgery patients)

What to expect after discharge to the ward and from hospital and

The help available to bereaved relatives.

A review of the accommodation available to relatives was also suggested. It was recommended that minimum requirements should include a waiting room, a separate room where bad news can be given (aside from a sister's or doctor's office) and overnight accommodation on or adjacent to the unit.

The report also recommended a review of staff communication systems. Following on from the Audit Commission's recommendations the Department of Health published Comprehensive Critical Care as part of a series of reports of potential improvement across many aspects of the NHS.

\subsubsection{Comprehensive Critical Care: $A$ review of adult critical care services. Department}

\section{of Health (2000a)}

This report was intended as a proposal for a modernisation programme for critical care services. Its aim was to develop a framework for future organisation and delivery of 
critical care. It proposed that patients' needs should be determined by the level of care their condition requires, not location of their bed and that responsibility for services should lie with Health Authorities and NHS Trusts. These services should be consistent, comprehensive, planned and delivered systematically across the whole NHS. The characteristics of this service are listed below:

1. An intergrated hospital wide approach extending beyond physical boundaries of the units, making best use of available services.

2. A service provided across all trusts, working to common standards and protocols.

3. A planned approach to workforce development including recriutment, retention and training to gain a balanced skill mix.

4. Ensuring practice is evidence based.

The report acknowledges the distressing nature of the environment for relatives and conscious patients. It recommends that the Trust-wide Critical Care Delivery Group should review the requirements for display material within critical care units. This material should describe the service and explain the purpose and operation of equipment. The Group should also take responsibility for ensuring a means of providing written information on topics such as; facilities available, descriptions of the staff likely to be involved in care, general information about the critical care service, important telephone numbers, relevant local and national organisations and chaplaincy services. It also recommended that reference be made to existing good examples whilst reviewing material. The report suggests that its recommendations be implemented within three to 
five years, i.e. by 2005 . It is currently unknown as to whether these recommendations concerning information provision have occurred. By acknowledging the importance of providing relatives with information these guidelines are attempting to encourage the treatment of the patient as a family unit. Addressing the needs and experiences of relatives has come to the forefront of many recent NHS reforms.

\subsection{The research study}

This study investigates relatives experiences of critical care from data gathered through three phases of qualitative and quantitative investigation. It compares the views and perspectives of relatives and qualified nursing staff and examines national provisions and policies for relatives within Department of Health guidelines.

\subsubsection{Rationale}

The literature in this area is sparse and a review revealed that the most prominent and influential study in this area to date is still Molter (1979) even though this is over two decades old. Published studies have concentrated on relatives needs and most have used the critical care family needs inventory (CCFNI) as an assessment tool, merely replicating previous findings and not advancing the research field (Dyer 1997). The CCFNI is a quantifiable survey measure that was predetermined by researchers without the consultation of relatives. Although a valuable tool, exact replication does not take in to account its limitations and thus the same flaws will appear in the subsequent research. There are few studies using qualitative methods and those that have still focus on relatives' needs rather than overall experience. Zainal and Scholes (1997) conducted one 
of the most recent qualitative studies using data collected in 1994, ten years ago. Their study looked at relatives experiences in relation to the introduction of primary nursing in the UK. This study aims to try and update the level of research in this area and address some of the gaps in the literature.

Furthermore, the majority of studies about relatives and critical care have been carried out in the United States. This may bias the findings and affect generalisability, due to different health care systems between countries. In the USA, the greater availability of beds and larger physician:patient ratio, means that more patients may be admitted to critical care (Ridley et al. 1996), resulting in a broader spectrum in illness severity in critical care patients, particularly with planned admissions. Consequently, needs of relatives in USA and UK may differ. Hence, such differences mean that results from one country cannot be directly applied to critical care practices of another. Also, the different healthcare system may influence care delivery, so it is important to carry out similar studies in other countries to distinguish between evidence that is related to critical care and evidence that is related to the health care system.

The Department of Health $(\mathrm{DoH})$ has developed a lot of recent initiatives concerning the NHS in general, such as The NHS Plan (Department of Health 2000b). It has also concentrated on specific areas of the health services one of which has been critical care (Department of Health 2000a; Department of Health 2001). It has not been investigated as to whether these initiatives are being implemented. In particular, recommendations have been made concerning information that relatives should be receiving while they are exposed to the critical care environment. These recommendations were made in 2000 and 
a time span of 3 to 5 years was given for the implementation. There is no evidence that indicates whether this has been followed up. This thesis aims to address that issue.

Communication and information are crucial within this environment. A lot of work has been put into examining communication between staff and patients however, little has been done with regard to communication between staff and relatives. Holistic care of patients has become more prominent in recent years, this includes looking at the patient as part of a family unit. In order to improve the experience for relatives and as a consequence enhance patient recovery, it is important to allow both relatives and staff to give their opinions on what their perceptions are.

\subsubsection{Research questions}

The overall aim of this project is to gain insight into relatives' experiences of critical care and how well staff perceive these experiences. The following research questions were formulated:

- What are relatives' experiences of critical care? How do staff perceive these experiences?

- Is there any evidence to suggest that relatives find these experiences psychologically traumatic?

- What information do critical care units provide for relatives nationally? Do units have policies concerning relatives' care? 
- Are Department of Health guidelines laid out in Comprehensive Critical Care: A Review of Adult Critical Care Services (Department of Health 2000a) relating to information provision for relatives being adhered to?

- Do differences exist between relatives' experiences in general critical care units and specialist neurological critical care units?

- What are relatives' specific views of the type, amount and nature of information they receive?

\subsubsection{Definition of Relative}

Many previous studies use the true definition of 'relative' and have only involved participants who are actual family members. Throughout this thesis 'relative' is defined as someone who is considered significant to the patient. This definition is not limited to blood relatives since others visitors could have similar experiences to relatives. It was also meant to reflect the increasing diversity of family structures within modern society. However, in reality, the participants in this study happened to all be actual relatives, as they were the people who visited the patient most frequently.

\subsubsection{The Research Process}

The research process underwent many stages. These are outlined in Figure 3. Each stage will be discussed in more detail throughout this thesis in the relevant chapters. 


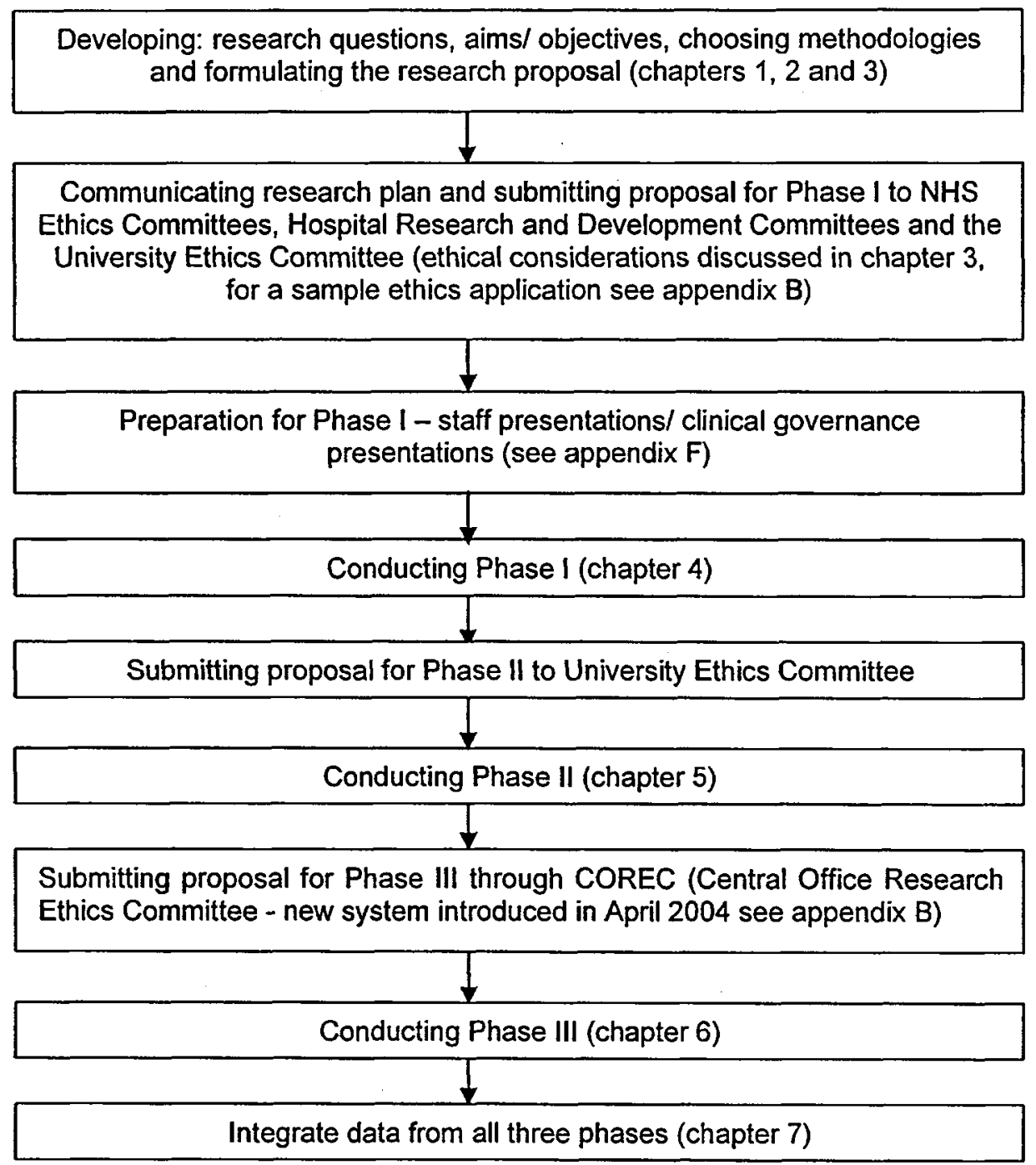

Figure 3- The research process

\subsubsection{Implications of the study}

Investigating relatives' experiences of critical care will contribute to knowledge in several ways. It will help to understand what relatives endure and what can be done to ease the transition from normality to crisis for those families. It will also help to identify staff involvement in the care of relatives and the extent to which this involvement fulfils the existing needs of relatives. 
Chapter Two

REVIEW OF THE LITERATURE 


\subsection{Holistic Approach to Care}

Critical care nursing is not simply about curing the critically ill patient. Increasingly, nurses are required to adopt a holistic approach, which involves 'caring for the whole person' (Castledine 2001), encompassing psychological well-being as well as patients' physical needs. For optimal care patients cannot be viewed as an individual entity. Generally, a patient is part of a family unit and, if true holistic care is to be achieved, the nurses and medical staff in a critical care unit must care equally for the relatives, and the critically ill patient. The concept of holistic care has existed for centuries and is particularly prevalent within healing traditions of the Far East, for example, Chinese medicine. Amongst western medical approaches it has never achieved the status it deserves (Castledine, 2001). More recently, holistic medicine has come to the forefront due to our inability to eliminate suffering and pain caused by modern illnesses such as cancer and AIDS, therefore are increasingly look for ways of alleviating, not eliminating, the problem. The NHS plan (Department of Health 2000b) increases the rights of patients and their families and states that "the NHS will shape its needs and services around the needs and preferences of individual patients, their families and carers" (Department of Health 2000b). This is reinforced in the Comprehensive Critical Care (Department of Health 2000a) report. However, despite this and despite nurses realising the importance of the family in holistic care of the patient, they are often reluctant to integrate the family into the critical care environment (Pryzby 2005).

Relatives of critically ill patients are under immense stress. Stress is the condition that results when interactions between a person and their environment lead the individual to perceive a discrepancy - whether real or not - between the demands of a situation 
and the resources of the person's biological, psychological and social systems (Cox 1988). Stress can exacerbate many situations, both physically and psychologically, for relatives and patients. A single stressful event may not place excess strain on an individual's coping abilities, but persistent, multiple stressors lead to coping abilities becoming overstretched and critical care is an environment that produces such multiple stressors. Dyer (1991) advocated that reducing stress levels of visitors, predominantly relatives, could lead to improvements in patient outcome. If visitors were less stressed they would visit more frequently and feel more comfortable with the situation when they did visit. This would be beneficial because visitors are more likely to provide staff with information about the patient which could help to improve individualised care. Visitors would also be in a stronger psychological state to provide emotional support for the patient, aiding patient recovery. In addition to the beneficial effects on patients, visitors who are less stressed and more at ease reduce the stress levels of medical staff, and in particular nursing staff, who interact with visitors most frequently. When carrying out research into relatives' experiences, to gain a balanced view of health service provision, the views of staff also need to be considered (Jennings 2001).

\subsection{A Crisis Situation}

There are two categories of patients admitted to a critical care unit; planned admissions and unplanned admissions. For the average general critical care unit $1 / 4$ of patients are planned admissions and $3 / 4$ are unexpected emergencies (Audit Commission 1999). Planned admissions arise when patients have elective surgery. In these situations patients and relatives are able to visit the critical care unit beforehand and are generally told what to expect after surgery, for example, the condition of the 
patient and the environment in which they will be nursed. Many cardiac centres in the USA offer such tours. A preoperative tour of the critical care unit is intended to lessen the anxiety of patients and families and improve their preparedness. However, evidence indicates (Lynn-McHale et al. 1997) that the preoperative tours of the critical care unit had no statistically significant benefit over the standard preoperative procedures, such as educational teaching and leaflets, in reducing anxiety. Regardless of the statistical findings, patients themselves reported that they perceived the tour was a beneficial experience. Lynn-McHale et al. (1997) observed that on the day preceding the cardiac surgery, relatives' anxiety levels were higher than those of the patient. It was also noted that relatives are often excluded from preoperative teaching. The unknown will only serve to increase anxiety and distress of the relatives.

Patients in critical care are often heavily sedated, either to ease pain, or to make it easier for staff to carry out certain procedures, resulting in the perception that patients are either in a worse condition than in reality, or giving the impression that patients are deteriorating. This perception can increase the distress of relatives' who are unused to seeing their family member in this condition.

On the other hand unplanned admissions can induce a crisis situation within the family who have not had time to psychologically prepare for the event. This creates a situational crisis that presents an overwhelming threat to the individual and their family. Twibell (1998) found that suddenness of admission was not related to coping effectiveness. The results were correlational, therefore causation cannot be inferred.

A crisis can either be viewed as a danger, whereby the family may be dramatically influenced by its negative effects on mental health, or an opportunity, because it means that the family are more receptive to therapeutic intervention (Woolley 1990). 
Caplan (1971) defines a crisis situation as 'when a person faces an obstacle...that is, for a time, insurmountable through the utilisation of customary methods of problem solving'. Succeeding this, there is a period of confusion and distress, resulting in many varied, fruitless efforts to devise a solution to the crisis. Woolley (1990) observes that the main avenues of social support in a crisis situation are immediate family members. When the cause of the crisis is hospitalisation of the family member this primary support has been removed. Thus, a relative may turn to nursing staff to restore their emotional equilibrium. In a crisis people do not have the spare emotional capacity to seek support, the support must come to them (Wilkinson 1995). Bowman (2000) advocates that in a crisis situation, relatives may have severe difficulty processing and retaining information because, as a person's level of arousal increases, the ability to process complex information decreases (Rosenzweig, Leiman, \& Breedlove 1996). This would be true of both relatives and patients, thus, the same information would need to be repeated in lay terms on a regular basis. Mirr (1991) found that despite the fact that family members could repeat the information given to them by medical staff, when asked the meaning of the information, it became obvious that it was not fully understood. Bowman (2000) suggested that a relative's ability to respond to the affected family member depends on the extent of their recognition of the crisis, their capacity to withstand stress and their capacity to use effective coping strategies. In addition, family conflicts can be highlighted by poor health and may be enlarged by critical illness (Lynch, Appelboam, \& McQuillan 2003). The ability to use effective coping strategies influences how people respond to situations and therefore may also influence susceptibility to psychological distress in adverse circumstances. 


\subsection{Intensive Care Unit Syndrome and Post Traumatic Stress}

Intensive care unit (ICU) syndrome was first observed at the advent of the critical care unit during the 1950's, when there were reports of 'acute mental abnormalities' in patients with poliomyelitis or patients who had undergone heart surgery (Granberg, Engberg, \& Lundberg 1996). The term was coined in 1966 and it is now widely acknowledged that patients who spend an extended period of time in critical care can suffer from ICU syndrome or ICU psychosis. The syndrome is caused by patients' psychological distress as a result of admission and symptoms consist of fluctuating levels of consciousness, poor orientation, delusions and hallucinations, behavioural abnormalities including aggression, passivity and non-compliance and memory disturbances (Bennun 2001). The incidence of the syndrome is indeterminate, ranging from 7 to $72 \%$ (Granberg, Engberg, \& Lundberg 1996). Factors affecting the onset of the syndrome may include the use of certain drugs, such as opioid analgesics, cortiosteriods and inotropics, together with the use of highly technological equipment and the age of the patient. Certain drugs can also cause psychiatric reactions and the brain of the elderly is more sensitive to the effects of certain types of drugs (Granberg, Engberg, \& Lundberg 1996). However, there is no single, specific, fundamental factor that causes ICU syndrome.

There is also evidence of other psychological problems such as post traumatic stress disorder (PTSD), which is one reaction to traumatic stressors. Symptoms include reliving the event via intrusive thoughts or nightmares, avoiding reminders and/or feeling emotionally numb and persistent feelings of anxiety. McMillan (2001) has conducted extensive research on the occurrence of PTSD following hospitalisation, specifically due to traumatic brain injury. It has been reported that $15 \%$ of patients 
admitted to critical care for periods of longer than four days suffered from PTSD nearly a year after discharge (Skirrow et al. 2001). The figure is large when compared to $1 \%$ of the general population, $19 \%$ in disabled war veterans (Gregurek 2001) and $3.5 \%$ in victims of assault (Jones, Griffiths, \& Humphris 1998). The percentage of psychological problems linked to critical care units vary, depending on the type of unit. The longer the admission, the higher the potential incidence of psychological problems. One of the most distressing factors of critical care units for patients is not being able to synchronise one's own breathing and this can lead to states of panic and insecurity (Bennun 2001).

A study on women with metastatic breast cancer concluded that women experienced clinically significant levels of intrusion and avoidance symptoms related to their cancer (Butler et al. 1999) which illustrates the impact that health and disease can have on psychological well-being. It was also observed that women who had suffered a greater number of stressful life events and high current levels of aversive emotional support, were more likely to suffer from clinical symptoms. Cancer is a stressful life event both for the patient and also for those close to the patient. It is possible that similar clinical symptoms could arise for relatives in critical care units, but it is an area that has not been investigated.

Using the criteria outlined in the Diagnostic and Statistical Manual of Mental Disorders (4 $4^{\text {th }}$ Edition) (American Psychiatric Association 1994), having a family member admitted to a critical care unit qualifies as a traumatic stressor. It is an event involving 'actual or threatened death or serious injury, or threat of physical integrity of self or others'. Therefore, the more the hospitalisation involves life threat, actual death, displacement from home or community, little or no warning, and exposure to 
bodily disfigurement or mutilation, the more severe a traumatic stressor it is (PeeblesKleiger 2000). Critical Care is possibly the most threatening unit in a hospital.

One explanation for PTSD is a deficit in cognitive-emotional processing (Rachman 2001). For effective emotional processing to take place emotional disturbances are absorbed to the point where behaviour and other experiences are not disrupted. If emotional processing is not effective, emotional activity becomes intrusive in the form of PTSD-like symptoms. Negative cognitions, such as those experienced whilst a loved one is in critical care, are thought to influence emotional processing. Delayed onset PTSD is problematic for this explanation.

Recently, memory problems have also been linked to emotional processing. Memory functioning plays a crucial role in psychological trauma. During a traumatic event the occurrence of intense arousal interferes with the information processing of the event (Turnbull 1998). As mentioned previously, a crisis results in difficulty in processing and retaining information. Traumatic memories are processed in a different way to non-traumatic memories, leaving them more easily accessible. The crisis caused by a family member being admitted to a critical care unit, may be a traumatic enough event to affect memory processes. This type of psychological trauma persists if the memory is classed as a current threat since, due to the method in which it is processed, it has no time limit (Ehlers \& Clark 2000). Autobiographical memories are processed verbally, in the past; they form a complete 'story' within themselves. Sensory memory traces have no time span and very little verbal input, hence the feeling of current threat.

Some relatives, with predetermined vulnerability factors, may be susceptible to developing a form of psychological trauma from the critical care experience. Jones et 
al. (2004), using quantitative methods, found a high incidence of psychological distress in patients $(n=104)$ and relatives $(n=104)$, which was not diminished by access to written information. To date, this is the only published study examining traumatic stress like symptoms in relatives as a result of critical care. Studies have been carried out examining anxiety and depressive symptoms (Pochard et al. 2001) which found that $69.1 \%$ of relatives suffered from anxiety and $35.4 \%$ from depression. However, this study also used quantitative methods.

The emotional maelstrom with which family members have to cope when visiting a patient in critical care make them particularly sensitive to suggestion. However, depression has been shown to be associated with viewing problems in dichotomous terms (Pochard et al. 2001). It is very likely that many family members suffer from temporary symptoms relating to the stresses of the event of the illness.

\subsection{Models of Coping}

Lazarus and Folkman (1984b) define coping as 'constantly changing cognitive and behavioural efforts to manage specific external and/or internal demands that are appraised as taxing or exceeding the resources of the person'. This definition limits coping to a person-orientated phenomenon, rather than the traditional trait-orientated views (Lazarus \& Folkman, 1984a). It also restricts coping to situations involving psychological stress, by referring to exceeding resources and does not suggest that coping can result in gaining total control of a situation; it merely implies that coping can lead to the ability to manage the situation. This management can involve minimizing the seriousness of a situation, avoiding or accepting the situation and attempting to control the physical environment. 
The traditional view of coping and emotion argues that the methods by which people choose to cope with a situation affects their emotional state. Research illustrates that emotion, predominantly anxiety, impedes cognitive function and, therefore, coping. Folkman and Lazarus (1988) advocated that emotion affects the coping strategy used, because people often experience more than one conflicting emotion at one time. The relationship between emotion and coping is bi-directional; each has an influence on the other. The appraisal process generates the emotion and it is this that affects the coping strategy. New encounters are reappraised leading to changes in the quality and intensity of the emotion. Figure 4 illustrates this process and indicates how coping acts as a mediator to emotion. Coping is generated during the encounter. 


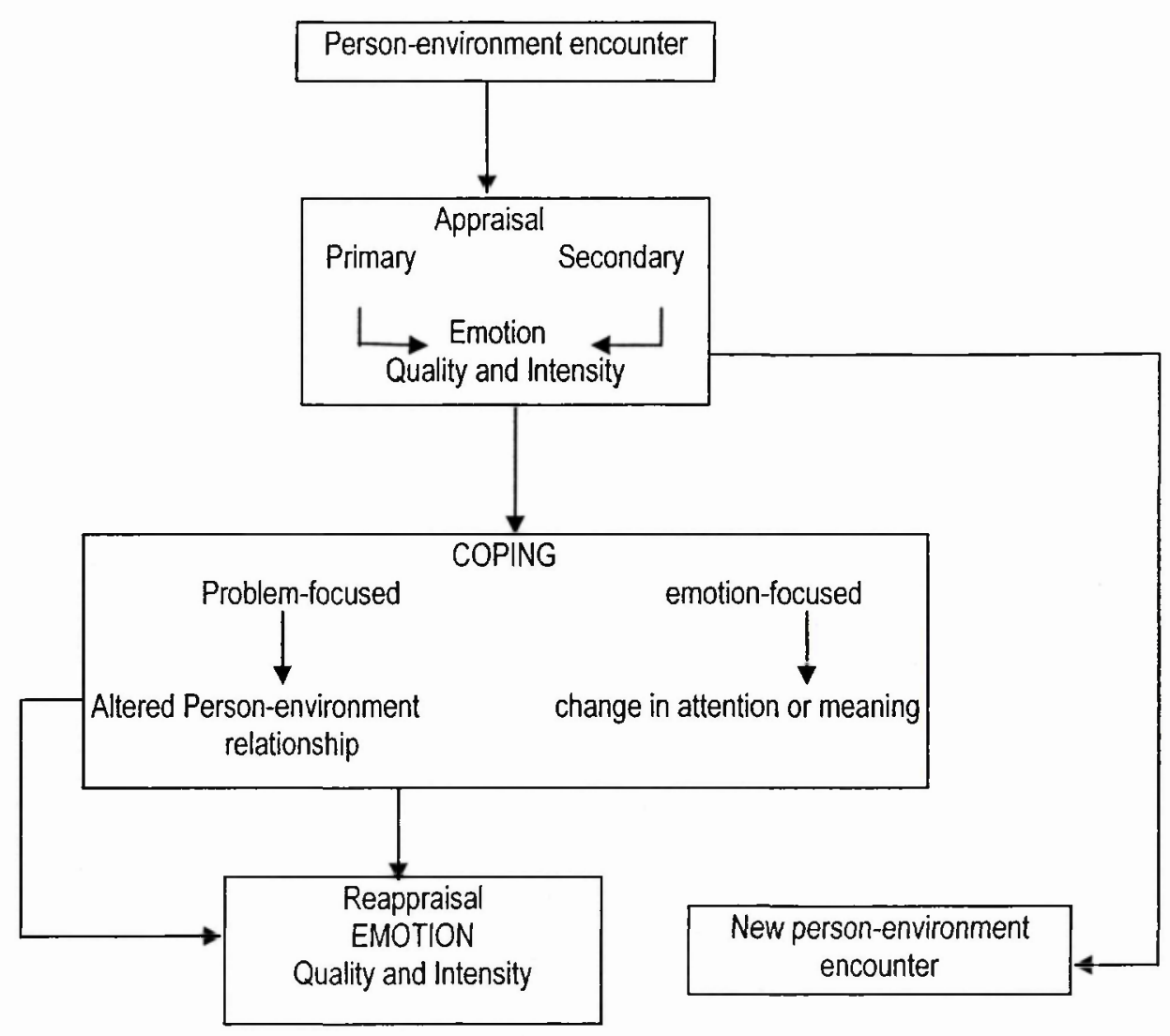

Figure 4-Coping as a mediator of emotion (Folkman \& Lazarus 1988)

\subsubsection{Direct Effect model vs. Buffering model}

There are two models that attempt to explain the impact of psychosocial coping resources. The 'direct effect model' presupposes that coping resources have a beneficial effect on psychological health, regardless of whether a stressor is present. The 'buffering model' advocates that the negative influences of stressors on health are alleviated by coping resources (Pennix et al. 1998). It is apparent that coping mechanisms are important in times of stress, however, information concerning for whom, from whom and under what circumstances these coping mechanisms exist appears to be lacking. Ineffective coping mechanisms are evident through various somatic anxiety symptoms, such as disturbed sleep patterns, weight loss, appetite loss, depression and lapses in concentration (King \& Gregor 1985). Breu and Dracup 
(1978) were the first to acknowledge a series of problems experienced by families who suffered an unexpected hospitalisation. These include:

- deprivation of primary social contact and major source of gratification and self-esteem;

- imposed autonomy;

- altered daily living patterns including sleep and meal times;

- role reversal;

- disjuncture of social contacts;

- financial instability through loss of income;

- interruption of interpersonal reward systems;

- relocation to an unfamiliar environment for most (possibly all) of the day .

The above factors can be applied to the critical care environment. If a patient is admitted to a critical care unit, communication with other family members will be disrupted. Therefore, a major avenue of support, and possibly companionship, has been lost, especially if that patient was pivotal to the functioning of that family, e.g. mother or father. This could also lead to role reversals, e.g. if a parent is in critical care, the child may have to assume many parental duties.

The environmental aspects of the critical care unit contributes to feelings of imposed autonomy, altered daily living patterns and relocation to an unfamiliar environment, 
entailing adjustment to visiting policies, spending extended periods of time at the unit and having to follow the procedures and policies of the unit.

Aspects such as financial instability become apparent at a later date, especially during rehabilitation periods when loss of earnings of the patient and/or the carer could become more pressing. This may increase stress and anxiety, which could in turn have the effect of hindering the rehabilitation process.

Social support is a major factor linked to coping but the process by which social support affects psychological and physical health has yet to be resolved. A possible hypothesis is that lack of positive social support induces negative psychological states, most commonly anxiety and depression. Psychological states in turn affect physical health, either directly through increased susceptibility to disease or indirectly through altered behavioural patterns that increase the risk of disease and mortality (Cohen \& Wills 1985).

The direct effect model postulates that social support is so influential because large social networks provide people with regular positive experiences. It provides an individual with a socially rewarded 'role' in the community, which promotes positive affect, stability and recognition of self worth. Being part of a social group helps to counteract negative experiences.

The buffering model claims that social support is one of the influential factors in easing the impact of a stressor. Figure 5 illustrates the two points in the causal chain between stress and illness where social support may play a part. Social support may intervene by preventing an event being appraised as stressful. Support at this stage may alter the perception of an event, or may boost one's perceived ability to cope. 
Alternatively, social support may intervene to prevent a pathological outcome, by reducing the stress reaction, or may even affect the physiological system directly.

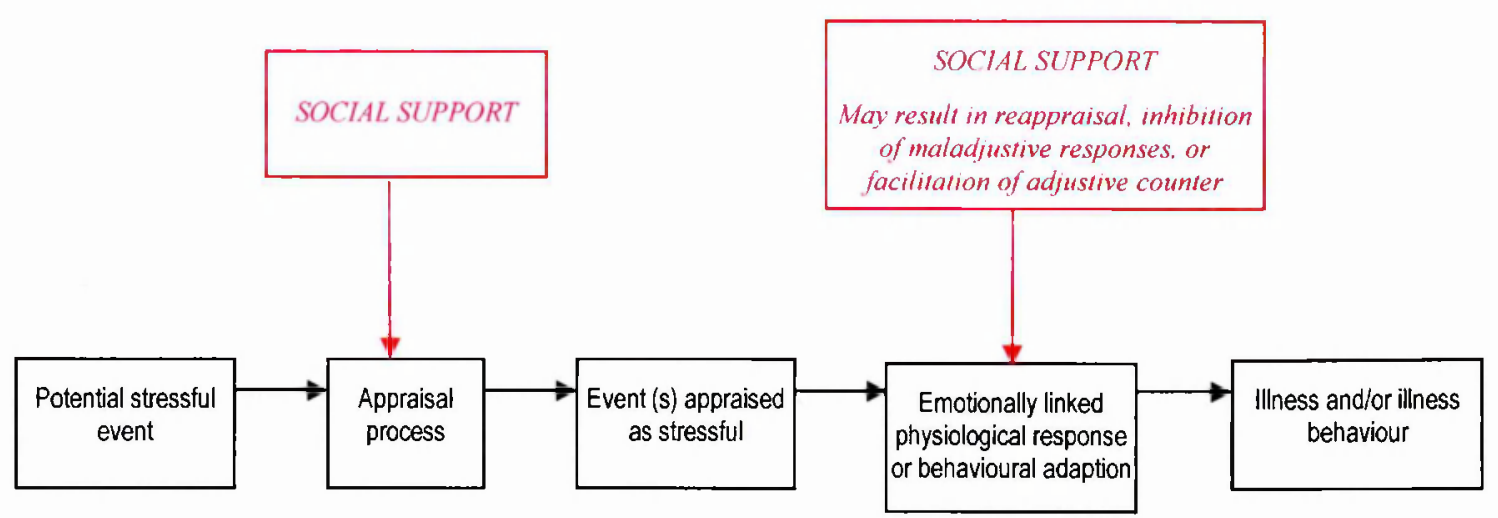

Figure 5- Possible stress buffering mechanisms of social support (Cohen \& Wills 1985)

Figure 6 demonstrates the different impact that social support has depending on the type of coping model used. Graph A illustrates the impact that social support has on the direct effect model. With increased social support there is a constant and consistent reduction in the number of symptoms, regardless of a specific stressor. Graph B shows how social support could in part reduce the effect of stress on symptomatology. Whereas graph $\mathrm{C}$ shows how social support can completely eradicate the effects of stress. However, support is not a factor in the buffering model until a stressor has actually occurred. For graphs B and C the stressor occurs at the interaction. 


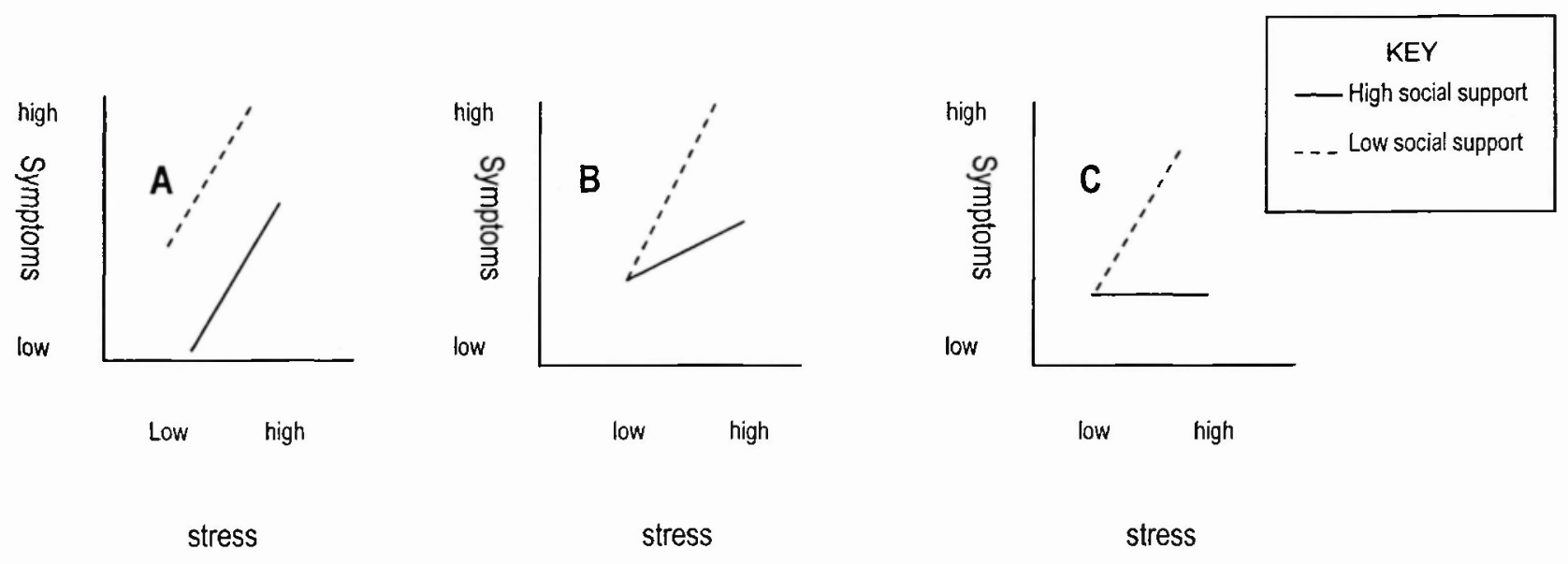

Figure 6-Graphs of direct effect model of social support on symptomatology and two variations of the buffering model of social support on symptomatology (Cohen \& Wills 1985)

There is considerable support for both types of models; however, the two do not appear to interact, despite evidence that they are not mutually exclusive concepts. Studies that discovered a significant buffering interaction and which gathered sufficient data to estimate whether there was a link between symptomology under low stress levels and social support, consistently produced a pure buffering effect. This finding indicates that certain support resources function only at increased stress levels. This hypothesis corresponds with the theory that specific support functions are responsive to stressful effects (buffering model), whereas social network integration operates to maintain feelings of stability and well-being, irrespective of stress levels (direct effect model) (Cohen \& Wills 1985).

\subsubsection{Emotion Focused-vs. Problem Focused Coping}

The buffering model and the direct effect model attempt to explain ways in which coping strategies in general influence psychological adjustment; most prominent of these strategies are emotion-focused coping and problem-focused coping. These 
strategies are not mutually exclusive. Coping is very much a process which is under constant re-evaluation, therefore, at the onset, an individual may adopt an emotionfocused approach and, after reappraisal of the situation, might at a later stage adopt a more problem-focused approach.

One of the main methods of emotion-focused coping involves using cognitive processes which lessen emotional distress, such as avoidance, minimisation, distancing, selective attention, positive comparisons and attempting to extract positive value from negative events. Another less common type of emotion-focused coping is used to increase emotional distress, reflecting an aspect of self-blame and punishment. An individual may need to make themselves feel worse before they can start to feel better. A similar, and similarly less common, cognitive strategy involves increasing emotional distress so that an individual is able to act, akin to the need to 'psyche oneself up' in order to carry out an act. Emotion-focused coping is also used in reappraisal, whereby people reduce emotional distress by changing the meaning of a situation, without the situation itself actually changing. This often reduces the threatening nature of a situation, for example in critical care reappraisal may occur in the form of 'at least he/she is still alive, it could be worse'.

A characteristic of coping processes is self-deception. Emotion-focused coping is used to retain hope and optimism and to deny worst-case scenarios; these strategies can distort reality and consequently deceive. However, for the strategy to work successfully the individual has to be unaware of its occurrence, since awareness causes self-deception to be ineffective (Lazarus \& Folkman 1984b).

Problem-focused coping uses cognitive strategies similar to those used in normal problem solving involving defining the problem, looking for solutions, weighing up 
the costs and benefits of the options, choosing an option and acting upon it. These strategies are more complex and less objective than straightforward problem solving and can also be directed inwardly. Two major types of problem-focused coping are those directed at the environment and those directed at the self. Strategies directed at the environment include altering, or attempting to alter, environmental pressures, barriers, resources and procedures. Strategies directed at the self include motivational and cognitive adjustments such as shifting goals, reducing personal involvement, finding alternative sources of pleasure, developing new standards of behaviour or learning new skills or procedures, all of which assist the person to manage or solve the problem.

Problem- and emotion-focused coping often occur concurrently and can both help and hinder each other. For example, if a person experiencing intense anxiety is able to regulate that anxiety they may be able to engage in the task at hand, thus further reducing the anxiety. Therefore, the problem-focused coping helps to reduce the ineffective emotion-focused coping. However, in some situations the reverse can occur and problem-focused methods only serve to increase emotional distress, perhaps because the method chosen has lead to more confusion or simply failed.

Despite the negative effects of stress and crisis situations, there is evidence that a person can experience positive psychological states during episodes of extreme stress (Folkman 1997). In a study on caregiver partners of men with AIDS, Folkman (1997) illustrates that many underwent positive reappraisals, despite the death or deterioration of their partner. These cognitive strategies reorganise a situation to present it in a positive manner. This may become apparent with relatives of a patient in critical care, but at present this has not been researched. Religion or a strong faith 
can ease negative reappraisals of chronic or severely stressful situations, helping to balance positive psychological states (Folkman 1997). The search for meaning is a common method of reappraising difficult situations in order to cope.

\subsubsection{Coping and Critical Care}

Lazarus (1966) was the first to identify three specific areas of critical care that caused psychological stress for the relatives of the patient. 'Disruption of community life' occurs through necessary visits to the patient and the communication problems encountered with a severely ill patient e.g. unresponsiveness of an unconscious patient. Breu and Dracup (1978) may have developed the previous list of problems arising from unexpected hospitalisation from Lazarus's theory. The possibility that the patient could die or suffer permanent disability was another factor. Relatives who fear the death of a family member may start the process of anticipatory grieving before the death occurs (Fulton, Madden, \& Minichiello 1996). This would be particularly acute in a critical care setting. Lazarus' (1966) final factor was uncertainty caused by the inability to control one's environment due to the unfamiliar technology that is intrinsic to the critical care setting. Woolley (1990) also notes that the dilemma of whether to leave children at home or the patient alone in hospital, can contribute to unnecessarily increased anxiety. There appears to be an inverse relationship between age and anxiety in family members (Twibell 1998), perhaps because older family members have had more time to develop and perfect coping mechanisms.

The critical care environment is highly stressful for all concerned. Studies have shown that it is equally stressful both for staff (White \& Tonkin, 1991) and for 
patients (Cornock 1998). It may therefore follow that it is also a stressful environment for onlookers, such as relatives. Staff are in this environment through choice, whereas relatives are not. Goodfellow et al (1997) claim that as critical care staff have chosen their profession they are able to deal with the accompanying stress and cope with it differently but more effectively than the normal population. There is some evidence that it is a certain personality type who chooses to work in critical care (Hudson 1993). Having a partner or children acts a buffer against the occupational stressors. Again this illustrates that social support reduces stress levels in another context. It would therefore follow that, if relatives have an adequate social support system, then their stress levels would be reduced, as demonstrated by either the buffering model or direct effect model.

Hope, despite potentially being a method of self-deception (see p 31), may also be another means by which relatives can reduce their stress levels and promote positive psychological states in order to cope more effectively with the highly charged critical care environment.

\subsubsection{The Concept of Hope}

Although there is no universal meaning of hope, one definition of hope is as a state of being, characterised by anticipation for a continued good state, or release from perceived entrapment (Miller 1991). It is an important contributory factor in effective coping strategies. Family members who talk as though there is little or no hope left seem to cope less successfully (King \& Gregor 1985) and are often more anxious, agitated and aggressive. Nurses touching and talking to an unconscious patient can indicate hope for relatives (King \& Gregor 1985). Families and patients are 
interdependent; if relatives lack hope, this can in turn impact on their patient's level of hope (Miller 1991) and as a consequence may affect the patients recovery. Hope ultimately protects people against the state of despair (Miller 1989).

Miller (1991) identifies two categories of hope; generalised and particularised. The former releases families, patients and nurses from expectations of specific goals or outcomes that are present for the latter. Patel (1996) explored the hope inspiring strategies used by spouses of critically ill adults $(n=20)$. Five sources of hope were identified: religion/ faith, significant others e.g. friends and family, personal character, health care professionals and devotion to the patient. The need 'to feel there was hope' has also been identified in studies carried out on relatives' needs (e.g. Coulter 1989; Molter 1979; Wilkinson 1995).

\subsection{Environmental Factors}

The alien surroundings of critical care do little to comfort a distressed patient. In spite of studies illustrating the demand for a more healing environment, little has been altered in the design of critical care units. The physical environment may also add to stress experienced by relatives making prolonged visits to the unit. The ultimate purpose of a critical care unit is to monitor patients and intervene quickly where necessary. They have minimal family input and the design is focused on medical intervention, to the extent that they exclude other important components of care (Jastremski 2000).

It is recommended that staff explain the uses of all machines to patients so that they feel reassured rather than frightened (Ashworth 1984). Relatives may experience the same, if not greater, fear of the physical aspects of the unit. An unconscious patient 
may not be immediately aware of their surroundings, whereas relatives who visit patients face a threatening environment from the outset. Therefore, the uses of machinery and the meanings of the alarms should be explained to them. Critical care staff are in the difficult position of having to balance the need for provision of the necessary care for the patients in a crowded hostile environment with the wish of the patient and family to spend more time together. In most critical care units, the bedside environment does not accommodate the constant presence of the family largely due to lack of space. Incorporating the family into the environment may reduce their anxiety and make the environment appear less hostile.

As a patient becomes more critically ill, the environment becomes increasingly hostile because additional machinery is required. Patients may be seen by new consultants and the family may visit more often, causing the patient's perceptual references to alter. The resulting erroneous perceptions of reality trigger misinterpretations leading to fear, apprehension, paranoia and confusion. These are some of the signs of ICU psychosis (Jastremski 2000). The heightened arousal means that the patient has difficulty processing information and, therefore, is less likely to understand what they are told and what is going on, leading to increased feelings of fear and confusion and thus creating a vicious circle (Rosenzweig, Leiman, \& Breedlove 1996).

\subsection{Communication}

Communication deficiencies have been highlighted as a source of increasing patient dissatisfaction with health care in the UK (Audit commission, 1993) The simplest definition of 'communication' is 'an act or action performed to impart or transmit information' (Ruesch \& Bateson, 1968). This definition holds no therapeutic value. 
Crawford et al. (1998) believe that the fundamental aim of nursing is to 'communicate care'. Bowman (2000) acknowledges that the most important task in critical care units is to focus on consistency, balanced communication and negotiation. Research suggests that nurses fail to communicate adequately with critical care patients (Llenore \& Ogle 1999) and there is little empirical evidence to suggest whether nurses communicate more successfully with relatives. However, this would be expected because a primary difficulty in communicating with critical care patients is their unresponsiveness. Peel (2003) advocates that it is unsafe to presuppose that the message converged by the sender will be interpreted in the same way when decoded by the receiver. Communication between nurses and patients and families have four aims (Potter \& Perry 2001):

- to establish a positive relationship;

- to give factual information;

- to determine needs;

- to optimise the use of resources.

Currently, the difficulties of communication have simply been documented and reasons for this failure remain unexplained. At present, theories as to why nurses fail to communicate adequately with critical care patients are from the viewpoint of researchers, not from the perspective of the nurses. If poor communication is explained from a behavioural perspective, with nurses' adapting in order to cope with stress, then the issue is likely to be more prominent in critical care units than in other wards. Research suggests that nurses suffering from high stress levels would not communicate as effectively as those with lower stress levels. (Llenore \& Ogle 1999). 
An second hypothesis as to why critical care nurses do not communicate adequately with their patients, is that poor communication is a character trait of the type of nurses attracted to 'high pace and high tension' work in an critical care setting (Hudson 1993). Llenore and Ogle (1999) observed that when communication did occur between nurses and patients, on the nurses' part it was mainly instructions, information and questions.

Active listening is a crucial element of all communication. It is usually the doctor who informs relatives of major changes in a patient's condition. However, because of extended contact with the patient and family, nurses may have more complete knowledge of the patient and are therefore in a better position than doctors to give relatives overall information and because of this contact they may feel more compelled to be realistic. In addition, many of the doctors treating a patient in critical care will be specialists in a particular area. If a cardiologist examines a patient's heart and diagnoses that it is healthy, he may tell a relative that the patient is improving, whereas, if a renal specialist examines the patient's kidney he may say that the situation is deteriorating, therefore there is potential for relatives to receive confusing information. Nurses attempt to provide a smooth path for relatives. If they are honest about the prognosis and the patient recovers, no one will feel cause for complaint, however, if they are over optimistic and the patient deteriorates the relatives may consider that a treatment overlooked, or was not carried out correctly.

Communicating with relatives would be facilitated if the family's perception of the patient's illness was explored and recognised, possibly disclosing deep beliefs that are embedded in a family's history, relationships, religion or culture and which could be resistant to medical definitions and realities (Bowman 2000). Turnock (1991) 
advocates that nurses fail to communicate with patients because initiating conversations may leave them open to awkward questions about the patients condition. Maguire (1992) noted that staff expressed a common fear that probing for information on how patients are feeling may cause them psychological distress. Fielding and Llewelyn (1987) claimed that administrative pressures prevented nurses from spending more time communicating with patients and may also be reflected in the time nurses spend communicating with relatives. They also believed that lower status nurses spent more time communicating with patients. It is not clear from their paper which hospital wards were used in their research. This may or may not be reflected in critical care units.

Hospital staff are well respected by society which may create an imbalance with regard to communication with relatives. A critical care environment is not conducive to balanced communication (Bowman 2000). Relatives may fall into the 'good visitor' role, whereby they feel unable to ask questions or contradict staff who are perceived as more knowledgeable, thus they do not 'rock the boat' (Hupcey 1999).

Green (1996) conducted a study concerned with patients' memories of critical care units and concluded that many patients remember more than staff realise and many reported hearing conversations that they felt were inappropriate. However this was a retrospective study with accompanying limitations, such as memory bias, memory gaps and retrospective rationalisations, rather than valid reports. Nonetheless, the results were consistent with other similar studies (e.g. Holland, 1997). Ashworth (1984) believes that good nurse-patient communication will help to overcome some of the problems created by the critical care environment. The nature of this communication involves a certain amount of emotional investment from the nurses 
and how this emotional investment is managed may affect the standard of that communication.

\subsection{Emotional Labour}

Emotional labour, first introduced by Hochschild (1983a) with respect to flight attendants, is a concept that has strong relevance to nursing (Smith 1992). It is defined as the induction or suppression "of feeling in order to sustain the outward countenance that produced the proper state of mind in others" (Hochschild 1983b). Jobs that involve emotional labour share three main characteristics:

(a) face to face or voice to voice contact with people

(b) a requirement of the worker to produce an emotional state in another person.

(c) a degree of control exercised by the employer over the employees, through training and supervision.

Emotional labour is a multidimensional construct (Zapf 2002) that involves managing emotions as part of a job. It is a particularly pertinent topic with regard to the care of patients' relatives. In all social interactions people play roles to attempt to create particular impressions. This is achieved by following certain societal rules (display rules) (Goffman 1959). People manage their emotions using a number of strategies, principally surface acting and deep acting.

Surface acting manages the visible aspects of emotion, whilst inner feelings remain unchanged, which can create emotional dissonance. Emotional dissonance occurs when, due to external demand, emotions are expressed in a particular situation which are not genuinely felt, creating internal conflict between the person and the role. 
Surface acting is not always satisfactory as often more than superficial emotions is expected. Conformity to social norms and display rules is not sufficient, particularly in a profession such as nursing, where patients and families want to feel as though the nurses genuinely care.

Deep acting is where individuals try to influence what they feel in order to become the role they are playing. Its origins are based in Stanislavski's concept of method acting (Stanislavski 1965) where "the actor's preparation is to cross the threshold of the subconcious...beforehand we have 'true-seeming feeling' afterwards 'sincerity of emotion'..." (p267). Expressive behaviour and inner feelings are both regulated.

There are both positive and negative aspects associated with carrying out a job with high emotional labour demands. Jobs involving emotional labour appear to provide greater job satisfaction and feelings of personal accomplishment. However, if appropriate support is not available for staff it can also lead to emotional exhaustion, depersonalisation and a variety of psychosomatic complaints and may lead to staff burnout. Contrarily, emotional labour can reduce potential negative effects at work, induce positive emotions and fulfil needs and expectations.

The 1:1 nurse:patient ratio found in critical care units necessitate nurses using more emotional labour than in general wards with a larger staff-patient ratio. Closer involvement with patients and their families and the life threatening nature of the environment mean nursing staff contend with increased raw emotion.

The Government promotion of patient-centred care (Department of Health 2000b) and holistic care, increases emotional labour because nurses are no longer shielded by task-orientated care methods. If poor communication is explained as an adaptation 
process by nurses, this would decrease emotional labour but increase emotional dissonance.

Bratt et al (2000) concluded that family issues and dynamics were the most frequently cited stressors among nursing staff in paediatric critical care units. Nursing staff stated that it was difficult dealing with parents who were strained, awkward and demanding, situations requiring large inputs of emotional labour and emotional dissonance. Paediatric critical care units have a different atmosphere because society percieves that it is less fair for children to be faced with critical illness. However, when dealing with certain relatives similar situations will still occur in adult critical care units.

Critical care nurses cited a range of stressors including high acuity levels, short staffing and interpersonal relationships (Bratt et al 2000). These factors could indirectly impact on the relatives' experience of critical care because they could be reflected in the nurses' behaviour towards patients and relatives.

It has been observed that problems experienced by nurses with management and doctors may be reflected in interactions with patients (Nievaard 1987). These problems could include issues stemming from the complexities of hospital organisation, e.g. allocation of resources and the distribution of power or problems arising from the differences in roles of doctors and nurses, particularly in critical care, which is a very nurse led unit that ultimately has to respond to the doctors in charge of each patient. This raises the further issue of whether what nurses' perceive as important for relatives is recognised and reflected by the management. Nievaard (1987) found a direct correlation between nurses' perceptions of their relationship with doctors and management and their attitude towards patients and patients' 
perception of the quality of care received. This may also reflect in their attitudes towards relatives and may in turn influence the experience that relatives have of critical care.

\subsection{Relatives' Needs and Experiences}

There is little information about relatives' reactions to critical care. Molter (1979) carried out the first published study into relatives needs. Relatives were given a list of 'need' statements, however, certain needs may have been omitted because relatives were not consulted in the compilation of this list, the Critical Care Family Needs Inventory (CCFNI). The highest ranked need was 'to feel there was hope', this also emerged as a prominent theme in a qualitative study carried out by Coulter (1989). Zainal \& Scholes (1997) also used qualitative methods to explore relatives experiences $(n=15)$. Their data in 1994, against the backdrop of the introduction of primary nursing in the UK, revealed the main themes as shock and emotional distress, the impact of the therapeutic setting, adapting through knowing and continuity and the concept of balancing hope and realistic expectations. In contrast Price et al (1991) found preservation of hope rated as a low priority.

Mendonca and Warren (1998) advanced Molter's work by attempting to establish whether the needs of the relatives were being met. Relatives $(n=52)$ were asked to fill out a CCFNI 24 hours after admission of their patient to critical care and a Needs Met Inventory (NMI) 36 to 48 hours after admission of their patient to critical care; only four of relatives ten most important needs were met. Greenwood (1998) noted the lack of duplication for critical care units in the UK. A key area of research in the UK is by Dyer (1991) who focused on whether staff could assess whether the needs of 
relatives were being met and not what those needs were. O'Neill-Norris and Grove (1986) suggested that families' perceptions of their needs differed from nurses' perceptions of family needs, e.g. relatives placed less emphasis on their physiological needs, such as eating and sleeping, than did nurses.

The studies to date have focused on the 'needs' of the relatives. None considered whether the reason for the patient being in critical care may affect how relatives react and cope with the situation. For example, it would be expected that the critical care unit would have less of an impact on a relative of a planned admission than on the relative of an unplanned admission. Similarly, different diagnoses may also impact differently on the relatives. A patient suffering from a neurological dysfunction may cause immense distress to the relatives, because the patient may act or respond with inappropriate or uncharacteristic behaviour. If patients develop brain damage they may not be perceived as the person that they were previously, which can be immensely difficult for the relatives to accept (Mathis 1984).

One study in America considered the differences in needs of relatives of patients with and without acute brain injury (Mathis 1984). Mathis found significant differences between her replication of Molter's (1979) study and Molter's original findings. Significant differences were found between the needs of relatives of patients with and without acute brain injury, however it was acknowledged that the method of data analysis could have produced a Type I error, thus, the results do not appear to be reliable, nor have they been established to be generalisable. In addition, this has limitations of cross-cultural generalisability. Since 1984, when Mathis carried out this study, which highlighted the problem of family separation and severely restricted visiting hours the critical care system has altered considerably. Many critical care 
units in the UK now have open visiting policies and provision for relatives to stay overnight although limitations on the number of visitors allowed at one time may be enforced to ensure that the patient receives sufficient rest. Despite official hospital policies of open visiting hours, Clarke (2000) observed that many nurses still restrict visits of family members, possibly to assert their control and protect themselves from the emotional demands generated by allowing the constant presence of relatives, particularly children.

The majority of research carried out on the needs of relatives in a critical care environment has been conducted in the USA, where the greater availability of beds and larger physician:patient ratio, means that more patients may be admitted to critical care (Ridley et al. 1996), resulting in a broader spectrum in illness severity in critical care patients, particularly with planned admissions. Consequently, needs of relatives in USA and UK may differ, hence, such differences mean that results from one country cannot be directly applied to critical care practices in the other. The needs of relatives have been examined in considerable detail and the results are largely supportive of each other. Many studies have used the CCFNI; none have concentrated on the specific 'experience' of critical care for relatives and the impact on their psychological well being. Subsequent research into relatives needs has used quantitative methods (Dyer 1991; Heyland et al. 2002) which focus on what happened not why things happen. In addition, much of this quantitative research has merely replicated Molters study using the same, or a very similar, version of the CCFNI (Dyer 1997). This does not necessarily expand the area of knowledge. As the first published study in its area, it seems improbable that Molter would have ascertained every need that relatives have, particularly as they were not involved in the 
compilation of the questionnaire. There is also the question of whether a need that is ranked as unimportant is really a need (Dyer 1997).

Most studies failed to mention nursing practices or unit characteristics that, potentially, could affect the needs and experiences of families e.g. overall unit philosophy regarding families, the role families played in the unit, visiting policy or location and conditions of the families' waiting area (Hickey 1990). The majority of critical care units have an open visiting policy, with the occasional exception of certain cardiac care units, where patients are considered to require increased levels of rest. It would be useful to establish the ease with which relatives are allowed to remain in the unit. Regardless of the visiting policy, relatives may feel uncomfortable remaining on the unit if they are made to feel in the way of the medical staff. Certainly, unlimited visiting hours places greater stress on staff (Bratt et al. 2000).

Following on from the Government Comprehensive Critical Care review, the Health and Social Care Act 2001 introduces the idea of 'discovery interviews.' This initiative was aimed to be launched by The Department of Health (2003) within critical care during 2004. The extent to which units have taken up the scheme is unknown. It was first piloted on coronary care patients and involves a clinician interviewing patients and/or relatives during the rehabilitation phase, to track the patient's history from the onset of the illness through treatment and follow up. The results of these interviews will be communicated to staff on the unit to develop small scale changes to practice. It is an individual, team by team, approach, so whether it can, or will, have national implications remains to be seen. 


\subsection{Summary}

It is apparent that the critical care environment impacts on general psychological well-being for patients, staff and relatives. It is the extent and significance of this impact that remains unclear and which requires further investigation. Difficulties associated with gaining appropriate access to critical care unit environments within NHS hospitals and ethical issues arising from contacting relatives in such stressful circumstances are probably major contributing factors to the lack of research in this area.

Whether critical care is an environment that does or does not produce psychological trauma in relatives needs to be established. Following this, issues surrounding how best to communicate with relatives, implementation of strategies to ease family crises when a patient is admitted to the unit and factors affecting people's coping abilities need to be addressed. Nurses are central to the critical care environment; they are an integral part of the support and communication networks and are in the best position to implement changes and highlight any problems.

Bowman (2000) suggested that a relative's ability to respond to the affected family member depends on the extent of their recognition of the crisis, their ability to withstand stress and their capacity to use effective coping strategies. If one element of communication is optimal use of resources (Potter \& Perry 2001), the family should be used as a resource. 
Chapter Three

RESEARCH PARADIGMS, METHODOLOGIES AND METHODS 


\subsection{Introduction}

The methods by which we collect data have a key effect on the extent to which research will allow critical analysis on the phenomenon under investigation (Habermas 1987). The most common research tool used in investigating relatives' needs is the Critical Care Family Needs Inventory (CCFNI). Because the area of relatives' experiences has not been well researched such questionnaires were considered inappropriate for this study. It was therefore decided that exploratory studies would be better suited to the subject area. Before a discussion of the qualitative study (Phases I and III), it seems expedient to first critique qualitative research within the context of the paradigm of naturalistic inquiry, from which it derives. It is anticipated that the ensuing discussion will demonstrate its appropriateness in the investigation of relatives' experiences. Phase II of this study used quantitative methods which will also be discussed.

\subsection{Research Paradigms}

A paradigm represents anembodiment of what we think about the world, but can not prove (Lincoln \& Guba 1985). Kuhn (1970) defined a paradigm as a world view about the phenomena under investigation, referring to the shared beliefs of members of a specific scientific community thus establishing the development of scientific knowledge. It is a philosophical approach rather than a methodological underpinning to the research.

The two main competing paradigms are the quantitative, or positivist, paradigm and the qualitative, or naturalist, paradigm. The former emerged from an empiricist 
tradition established by influential people including Mill (1806-1873), Newton (16421727) and Locke (1632-1704). The latter emerged as a counter movement in the late nineteenth century by philosophers such as Weber (1864-1920) and Kant (1724-1894) (Creswell 1994). These paradigms make different assumptions based on ontological, epistemological, axiological and methodological approaches.

Ontology is the branch of metaphysics that deals with the nature of being or the nature of reality (Denzin \& Lincoln 2000). Quantitative researchers advocate that there is only one reality which is objective and independent of the researcher. Qualitative researchers hold that there are multiple constructed realities which are formed by those involved in the research i.e. the researcher, the participants and the audience. Naturalistic inquiry aims to understand the lived world or reality of the participant (Creswell 1994).

Epistemology is the branch of philosophy concerned with the origins, nature, methods and limits of human knowledge. The positivist approach supports the view that the researcher is independent from the researched. In quantitative research, researchers try to control bias, choosing systematic samples and remaining objective when assessing situations. Conversely, the naturalistic tradition believes that knowledge is constructed. Naturalism holds the view that the researcher and the researched are interdependent and therefore cannot be totally separated, the latter is subjective to the views of the former and values mediate and shape what is understood. These beliefs lend themselves to qualitative methodologies and methods. Within qualitative research the researcher acknowledges their values and bias, together with those contained in the information from the participants (Denzin \& Lincoln 2000). These viewpoints implicate the axiological assumption of the roles of values within the 
research study. Values are removed from quantitative research, so that it remains unbiased. In contrast, qualitative research is value laden and biased (Creswell 1994).

From the above distinctions different methodologies have emerged for the two paradigms. A research methodology is a theoretical analysis for defining a research problem and establishing how the research should proceed. Quantitative methodology uses a deductive form of logic to test theories and hypotheses to establish cause and effect. Concepts, variables and hypotheses are determined before the study commences and remain unchanged throughout. Qualitative research is a form of social inquiry that uses descriptive data, i.e. the participants' own written or spoken words or observable behaviour, to investigate the way people interpret, and make sense of, their experiences and their surroundings (Holloway 1997), the aim of which is to gather descriptions of the research topic from the participants' perspective about it. Qualitative research uses inductive logic; concepts, insights and understandings are developed from patterns that emerge from the data (Strauss \& Corbin 1998). It is not the case that one paradigm is superior to or more scientific than another, merely that they both contribute different qualities for differing purposes. This thesis adopts both quantitative and qualitative methodologies. Figure 7 outlines the research plan and illustrates the three phases of the study and the methods and paradigms adopted. 
PHASE

METHOD

PARADIGM

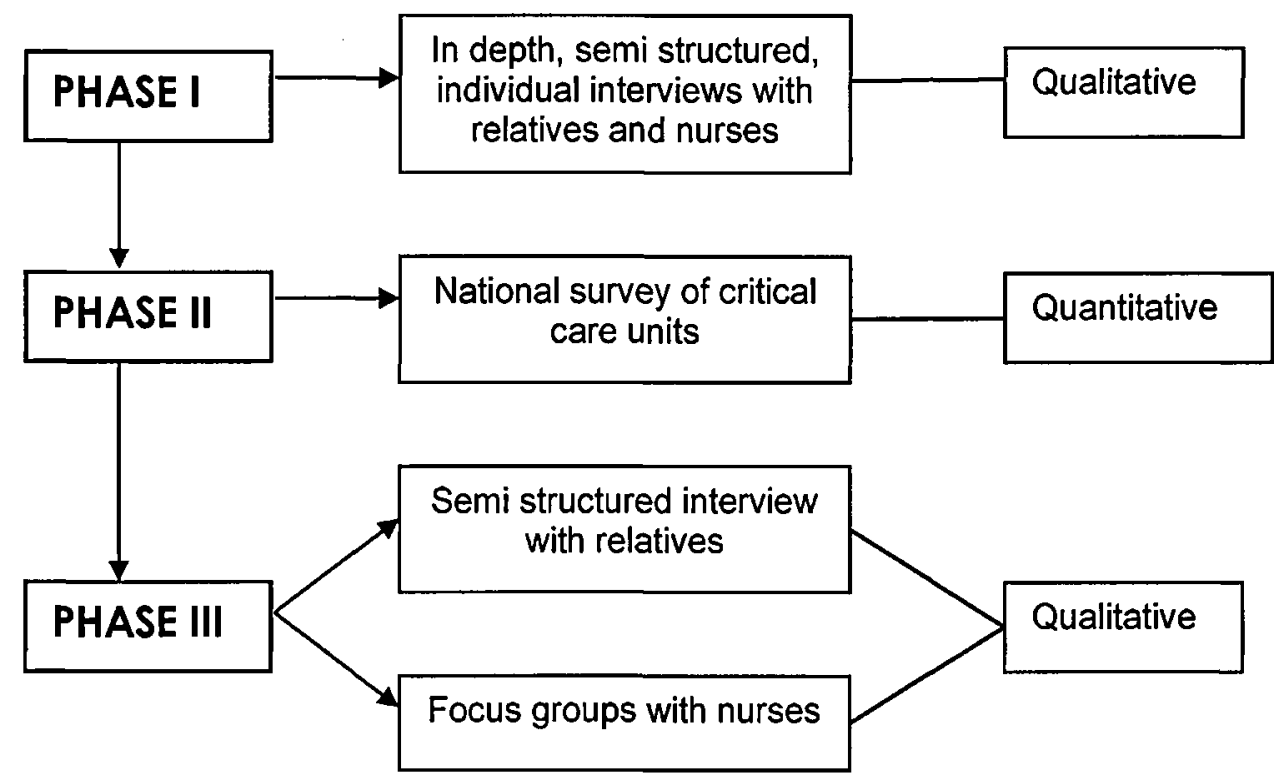

Figure 7 - The research plan

\subsection{Qualitative Research}

Qualitative research emphasises the importance of understanding the meaning of experience, events and actions, from the point of view of the participants as they naturally occur (Lincoln \& Guba 1985). It does not aim to test hypotheses (Pope \& Mays 1995), but humanises the research process by raising the role of the researched.

Qualitative research, concentrates on the 'how' not the 'why' and can be viewed as a holistic form of enquiry, allowing greater flexibility. A critical advantage of qualitative research is that it seeks to understand a phenomenon as a whole, not the sum of its parts and, as such, develops a deeper understanding of the respondent's world. The primary aims of qualitative research are to study reality from the inside and give a voice to the participants. It also aims to gain an insight into the perspectives of the participants; to develop a more realistic view of the world. It does 
not, however, aim to measure these perspectives. The ultimate objective is to explore and gain insights into new fields by producing descriptive data and developing concepts and theories. Its strengths are based on being naturalistic and inductive; it is grounded in a context and produces non-numerical results.

Qualitative research is valid and has the potential to discover new areas of investigation. It is able to identify patterns, reveal and explain complexities and enables the development, construction and testing of concepts and theories. Some perceived 'weaknesses' of this type of research relate to its very nature and reflect a traditional positivistic prejudice of assessment (Sarantakos 1998). Qualitative research is a unique type of academic activity which should be assessed in its own context.

Qualitative methods are believed to answer a number of reservations arising from uncritical use of quantification, by addressing the problem of inappropriately assigning meanings where these are variable and negotiable, in relation to their context of use. Using such methods can reduce the problem of overwriting internally structured subjectives with a priori systems of meaning (e.g. as occurs with standard survey instruments). Weaknesses of qualitative research include problems of reliability caused by extreme subjectivity and the risk of collecting meaningless and irrelevant information during the course of less structured interviews (Sarantakos 1998). The process of collecting and analysing qualitative data is extremely time consuming and findings of such research may produce problems with representativeness and generalisability. The research process may encounter difficulties in objectivity and attachment, and an awareness of ethical issues raised by entering the personal sphere of participants is essential. The flexibility of qualitative research makes it particularly well suited to exploratory or hypothesis generating enquiries. However, the smallness of the sample is sometimes deemed a disadvantage. 


\subsection{Evaluation of Qualitative Research}

A researcher must persuade their readership that their investigation is worth implementing and their results are worth consideration. For this to occur the reader needs to trust the research. In turn, this dependability limits the likelihood of an investigation being erroneous. Some researchers maintain that evaluation of qualitative research using the same canons or standards as quantitative research is inappropriate. The traditional criteria for measuring quantitative research were based on the four premises of internal validity, reliability, objectivity and generalisability. Lincoln and Guba (1985) established a more suitable set of criteria to evaluate qualitative research that are not the same as, but refer to, the above criteria. These are the canons of credibility, dependability, neutrality and transferability which are discussed below.

Credibility relates to truthfulness and internal validity. In order to achieve a truth value the researcher must execute the study in such a way that the probability that the findings will be found credible is maximized, which will largely depend on the researcher's skills during data collection and analysis. Methods of ensuring credibility include prolonged engagement, persistent observation, and triangulation (Lincoln and Guba, 1985). Prolonged engagement necessitates investing sufficient time to achieve certain purposes e.g. understanding and familiarization with the environment and trust building. Persistent observation adds depth to the process of prolonged engagement by identifying features and elements in the situation that are most relevant to the problem or issue being studied. Triangulation refers to the combination of two or more methods, theories, data sources or investigators in one study (Gilhooly et al. 2003), the purpose of which is to reduce researcher bias and increase validity. The 
researcher looks for patterns of convergence to develop an overall interpretation (Mays \& Pope 2000). The different methods of triangulation are outlined in table 2.

\section{Table 2 Summary of different processes of triangulation}

\begin{tabular}{|l|l|}
\hline Type of triangulation & Description \\
\hline Method & $\begin{array}{l}\text { A combination of research methods or } \\
\text { data collection techniques. For example } \\
\text { combining qualitative and quantitative } \\
\text { methods. } \\
\text { Different data sources with similar focal } \\
\text { point to obtain a range of views through } \\
\text { varied data about a topic e.g in this study } \\
\text { approaching both relatives and staff. }\end{array}$ \\
Tnvestigator & $\begin{array}{l}\text { Multiple investigators are used as part of } \\
\text { a team with adequate communication } \\
\text { between them to keep all members } \\
\text { moving together. } \\
\text { Different theoretical perspectives are } \\
\text { combined. A viewpoint not accepted as } \\
\text { viable in naturalistic enquiry. }\end{array}$ \\
\hline
\end{tabular}

Phases I and III of this thesis adopted data triangulation by seeking the views of both relatives and staff. Phase III also involved method triangulation by collecting data using both interviews and focus groups. In addition, credibility can be enhanced by the inclusion of negative or deviant cases, which challenge the researchers interpretations or contradict the evidence (Mays \& Pope 1995).

The second canon of evaluation is dependability, which denotes consistency or reliability. This centres upon the research process and ensures that, given the same theoretical perspective of the researcher, following the same general rules for data gathering and analysis and assuming a similar set of conditions and similar 
participants in a similar context, the findings would be repeated. This bestows credibility on original findings (Strauss \& Corbin 1998). The researcher has a responsibility to prove that the research process is consistent, logical, traceable and documented. Variance will occur within naturalistic inquiry, but that variance must be traceable. Ways to augment dependability include clarity of questions, collection of data across the full range of appropriate settings, sampling, use of appropriate recording equipment and keeping detailed notes.

The third criterion is neutrality which implies objectivity. This ensures that the findings of an investigation do not warp the reality they are meant to describe (Schwandt 1997) and confirms that the findings were grounded in the data (Hamberg et al. 2005). It enables the development of themes and concepts to be tracked to their sources, but does not imply that the results must have no researcher bias. This can be verified by an audit trail.

Finally, qualitative research is evaluated on transferability. This refers to the applicability of the results of a study and to what extent they can be applied to other contexts or with other participants (Lincoln \& Guba, 1985). In the case of qualitative research this depends on the similarity of the contexts being investigated and means that the researcher must provide the reader with sufficient information on the study to determine whether the findings are relevant and transferring the results is appropriate (Miles \& Huberman 1994).

\subsection{Qualitative Methodologies}

Many methodologies adopted by qualitative research share similar features. These include open research questions, rigorous but flexible designs, small participant 
numbers, purposive or theoretical sampling, no statistical analysis and the researcher being instrumental in collecting data analysis and interpretation. Methodologies include grounded theory, phenomenology, ethnography and hermeneutics. These methodologies are not the subject of this review and are described by Denzin and Lincoln (2000).

This study predominantly adopted qualitative methods and grounded theory methodology. Because of the lack of research in the chosen area, qualitative methods seemed the logical procedure to adopt and were selected because of the capability of identifying patterns and revealing new areas of research. In this instance, the primary advantage of qualitative methods is the ability to construct and test concepts and theories in order to generate a hypothesis. A more detailed discussion of specific qualitative methods can be found later in this chapter.

\subsubsection{Grounded theory}

Grounded theory is particularly suited to the study of local interactions and meanings of the social context in which they occur (Gilhooly et al. 2003). It uses the process of constant comparison and theoretical sampling to generate theory. The conceptual categories that are formed from the data generate the theory and, in turn, these data are used to illustrate the categories. Throughout this thesis quotations were chosen because they represent the themes most appropriately. Grounded theory can be applied to any qualitative methods but the most commonly used are interviews.

Constant comparison and theoretical sampling are more than data processing procedures; they build a conceptual and theoretical depth to the analysis. Throughout the span of the research study the data are constantly sorted and compared for 
similarities and differences at all levels e.g. basic data instances, cases, emergent categories and theoretical propositions, to generate conceptual categories.

Theoretical sampling is the active sampling of new data as the analysis proceeds. The analyst decides what data to collect next and where to find them, in order to develop the theory. Participants are chosen because they know most about the experiences being explored and will contribute most to the emerging theory (Strauss \& Corbin 1998). Additional data are collected to sharpen the definition of the concepts and to define their properties. Negative case analysis may also occur in theoretical sampling, whereby cases are explored that do not appear to fit, thus challenging and strengthening assumptions. The sample size in qualitative studies is small compared to quantitative studies; six-eight participants for homogenous samples and $12-20$ for maximum variation (Zyzanski et al. 1992). Data collection continues to the point of saturation i.e. whereby additional data add no new information.

Simultaneously collecting (interviews), coding (including compiling notes and memos about key concepts and their relationships) and analyzing the qualitative data is fundamental to generate the theory; definite separation of each would hinder the operation (Glaser \& Strauss 1967) thus, the traditional distinction would disintegrate. Data analysis should commence as soon as there is an adequate amount of material to analyse, and the subsequent analysis feeds back into sampling new data. Theories are formed by proposing plausible relationships between sets of concepts. These principles were selected because they are specific, highly developed and rigorous set of procedures for generating theory through comparative analysis and triangulation (Sarantakos 1998). 
Open coding is the first analytical step and is the start of the process by which concepts are identified and their properties and dimensions are discovered in the data (Strauss \& Corbin 1998). During open coding, data are broken down into discrete parts, examined and compared for similarities and differences. Parts that emerge as conceptually similar in nature and of related meaning are grouped under more abstract concepts termed categories. In subsequent analytical steps, such as axial and selective coding, data are reconstructed through statements about the nature of relationships. Open coding is performed line by line; a time consuming procedure but one which allows the analyst to generate categories quickly and to develop those categories through further sampling.

Axial coding is the second analytical step and refers to the process of relating categories to subcategories. This links categories through properties and dimensions and attempts to answer questions such as 'why'? 'where?' 'when?' 'how?' 'with what?' in order to relate structure with process. Structure and process are intrinsically linked and, unless the nature of their relationship is understood, it is difficult to comprehend what is happening. Axial coding is an important step to developing theory. Finally, selective coding is the process of integrating and refining theory. Categories are organized around a central explanatory concept; a process that happens over time. The theory is validated by comparing it to the raw data or presenting it to respondents for their reactions (Strauss \& Corbin 1998). This study used the former method. Theories are formed by suggesting plausible relationships between categories and a tentative hypothetical explanation is suggested. By studying the data, case by case, it is determined to what extent the hypothesis is accurate. As the concepts emerge the hypothesis is revised to fit the cases and is accordingly continually amended. By following this procedure through a number of cases the final theory 
should have greater explanatory power (Strauss \& Corbin 1998). A limitation of the methodology is the inability to study all relevant cases, with the resulting possibility of missing important data. This can be minimized by choosing a representative sample by theoretical sampling.

Collecting, coding and analysing the data was carried out simultaneously, in accordance with grounded theory. As the process of theoretical sampling was carried out the interview guides were revised to enhance data interpretation and to identify emerging themes and theoretical saturation. Results were fed back to those critical care units that participated in the research.

Criticisms have been raised concerning issues of reproducibility and generalisabilty. Whilst it is possible to accurately reproduce the methods, this may not lead to identical results and, therefore, the results cannot be generalised. These results are dependent on the sample from which they were drawn and from which a set of hypotheses can be developed that can then be tested on the population at large (Strauss \& Corbin 1998). Using grounded theory methodology makes it possible to minimize risks, as scientific rigour is ensured by the checks that are part of the data collection and the different phases of analysis (Strauss \& Corbin 1998).

\subsubsection{Data Analysis}

The transcripts were prepared for analysis and coded using QSR NUD*IST 6 (N6) (Qualitative Solutions and Research 2003), a qualitative software package. Nonnumerical Unstructured Data Indexing, Searching and Theorizing (NUD*IST) allows the user to manage, explore and search the texts imported into the programme, manage and explore ideas and link and construct theories about the data, test theories, and generate reports. It is an aid to analysis rather than a replacement for it. The 
interviews were imported as text files into N6 to be coded. In N6, data are coded in units of text: paragraphs, sentences or lines. In this study, data were coded line by line. N6 is used to code and re-arrange the data as themes emerge and build a hierarchical taxonomy, whilst simultaneously keeping memos with the raw data.

In order to become familiar with the data, the transcriptions were read and re-read. Interviews were summarized to identify patterns and themes that reflected the experiences of the participants. As a second step, the transcripts were coded line by line to give an initial coding scheme. Each line was given a label that described the main theme in that line. Each label was defined. Once every line was labelled, the labels (or codes) were examined for repetition i.e. labels that had the same meaning. If repeated, codes were merged to form a single label. These refined labels were the lower level themes, for example, fear, shock. Lower level themes and their definitions were compared and relationships between themes were identified. Lower level themes that were related were grouped into higher level themes, e.g. emotional reactions, and higher level themes were grouped into categories, e.g. relatives, to give a hierarchical taxonomy. A coding guide was developed to standardise the coding procedure. Analysis was an ongoing process i.e. transcripts were analysed before more data were collected.

\subsection{Qualitative Methods}

\subsubsection{Research Interviews}

Interviews are the most commonly used qualitative technique in the health care environment (Britten 1999) and were used in Phases I and III. There are three main types of research interview; structured, semi-structured and depth. Structured 
interviews primarily involve administering questionnaires and interviewers are trained to ask questions in a standardised fashion. Semi-structured interviews have a loose structure involving open-ended questions that define the area being investigated, together with probes and prompts and was the type of interview used in this thesis. Depth interviews are the least structured and may only cover one issue, but in greater depth. Research interviews enable information to be obtained and develop an understanding of issues relevant to the general aims and specific questions of a research project (Gillham 2000). The degree to which interviews are structured depends on the research topic and purpose, resources, methodological standards and preferences and type of information wanted, which in turn is determined by the research questions. Interviews are also defined by whether they are standardised or unstandardised. Standardised interviews use a set of response categories e.g. Lickert scales, whilst unstandardised interviews are characterised by open responses. Interviews can be both a quantitative and qualitative method. Quantitative interviews are largely structured and standardised.

Qualitative interviews allow access to the subjective world. They use open ended questions only and offer more flexibility to the interviewer in presenting the questions, changing the wording and order and adjusting the interview to meet the aims of the study (Sarantakos 1998). Qualitative interviews employ methods and a process of analysis that reflect the nature of the research object rather than the methodological conviction of the researcher resulting in the interview schedules being revised as more data became available.

Concepts and variables that emerge may be at variance with those predicted at the outset and the research needs to remain open to this possibility e.g. in phase I indications that relatives found their critical care experience psychological distressing 
did not emerge, whereas communication issues did. The interviewer must interact with the interviewee and be receptive to the language and concepts used. The interview process must be flexible and interviewers should confirm that they have understood the participants' meaning rather than using their own assumptions. This flexibility means that interviews can be adjusted to meet many diverse situations. Questions should be open ended, neutral, sensitive and clear (Britten 1999). Conducting qualitative interviews is demanding and time consuming and the interviewer must not lead the interviewee. However, interviews are perceived as collaborative tasks rather than the sole responsibility of the interviewee and, as such, tend to have a higher response rate and require less motivation on behalf of the interviewee than filling out a questionnaire (Sarantakos 1998). Interviews also allow the interviewer a certain amount of control over the environment in which they are conducted and allow the opportunity for the interviewer to correct or clarify any misunderstandings the interviewee may have and enables more complex questions to be attempted. Interviews allow spontaneous answers as respondents do not have time to reconsider their responses. Interviews are also a way of ensuring that the person to whom the questions are aimed is actually the person who responds which cannot be guaranteed in postal questionnaire measures.

Interviewer bias can be deemed a weakness of interviews as a method. In addition, people often act in different ways to how they say they will act. The trustworthiness of interview data should not be taken at face value but understood in the context in which it was obtained. 


\subsubsection{Focus groups}

Focus groups are essentially group interviews. They are a qualitative data gathering technique (Denzin \& Lincoln 2000) wherein the interviewer directs the interaction in either a structured or unstructured manner depending on the purpose of the focus group. Focus groups were carried out with critical care nursing staff with a predetermined agenda to gain specific information from the participants in Phase III of this study. Focus groups get closer to participants' understanding of and perspectives on certain issues, in this case communication between relatives and staff in critical care units.

The skills required by a group interviewer do not differ greatly from those required by an individual interviewer, however, certain additional skills are required. The interviewer must prevent one person, or a small proportion of the group, from dominating the session and encourage quieter members of the group to participate fully. Group dynamics need to be carefully managed and to get the most out of a focus group responses from the entire group need to be obtained.

There are advantages and disadvantages of using focus groups. Advantages are that it is inexpensive and an effective use of time for participants who are difficult to access. Focus groups were chosen for Phase III because it was considered to be the most effective use of time for critical care staff and enabled the views of a larger number of staff members to be assessed. It is data rich and flexible and stimulates the participants by encouraging them to 'bounce off each other.' People are believed to become more aware of their own opinions when faced with those who disagree (Breakwell, Hammond, \& Fife-Schaw 1995). Focus groups are cumulative and 
elaborative over and above individual responses. They build upon the data gained from previous individual interviews with nurses in Phase I.

Disadvantages of using focus groups include the possibility of 'group think' (Janis 1982), domination of the group by one person, difficulty in researching sensitive topics within a group and issues of confidentiality and anonymity which cannot be completely assured. In this study there is the danger that nurses may feel that by saying something it may undermine their own professional competence and to minimize this possibility the focus groups were kept small. A pilot focus group was carried out prior to the start of the main study to ensure that, in the circumstances, it was a workable method. Results from the Phase I interviews were fed into the focus group and the nurses in the group talked freely. It was deemed a successful method to use and therefore focus groups were carried out in Phase III.

\subsection{Quantitative Research}

Traditionally the steering values of sound research in western science are detachment, objectivity and rationality (Gilhooly et al. 2003). From this starting point, quantitative research can be associated with measuring, manipulating and specifying causal links between specific variables to test hypotheses. Qualitative research and quantitative research are based on an interpretative paradigm and a positivist paradigm respectively. Quantitative research is based on the premise that reality is objective, simple and positive and consists of sense impressions i.e. there is only one reality. Quantitative research is deductive and its results produce numeric, data and which is often generalisable, reliable and realistic (Sarantakos 1998). 
Human beings are subject to fixed patterns that govern their social world and these laws are empirically observable. Quantitative researchers believe that facts are separate from values and that it is not the researcher's job to make value judgments. All science, both natural and social, shares common logical and methodological foundations and, as such, should use the same methods. Explanation is derived exclusively from experience and is restricted to positive phenomenon. Quantitative research aims to establish law like causal statements about generalised behaviour. It is a closed approach that is strictly planned from the outset.

\subsection{Evaluation of Quantitative Research}

With the advancement of qualitative methodologies, quantitative research has received much criticism, particularly within the realm of social research. As previously mentioned, the cannons of evaluation for quantitative research are validity, objectivity, generalisability and reliability.

Validity, in general terms, refers to the property of being true, correct and conforming to reality (Reber 1995). There are many types of validity: Internal validity is an informal procedure for determining the validity of a test by assessing the degree to which it is fulfilling its intended role. It is concerned with whether the research design adequately controls extraneous variables and eliminates plausible rival explanations for the research findings. Internal validity is related to credibility in qualitative research. Construct validity or objectivity, asks whether there is evidence that the study succeeded in measuring the attributes or variables the researcher intended to measure. The meaning of objectivity derives from the notion that reality is observable and therefore publicly verifiable and not based on internal subjective experience. External validity is concerned with whether the research employed a sampling 
strategy that allows for the generalization of the results beyond the specific research participants, research setting and time period. If the results can be generalized it should be acknowledged to whom and in what setting. A major criticism of quantitative research is that reality is subjective and therefore not generalisable.

Reliability refers to whether there is evidence that the data collection instruments used provided accurate, consistent, repeatable and stable measures of the attributes or variables the researcher intended to measure. Reliability of measurement reduces influence of researcher bias.

\subsection{Quantitative Methods}

\subsubsection{Surveys}

Surveys and sampling techniques are not intrinsically linked to a particular philosophical standpoint. Appropriate sampling techniques are needed when whole populations cannot be approached in order to ensure that the results will be generalisable. Although structured surveys and questionnaires are most common, it is still possible to collect qualitative data using survey methods. Cross-sectional surveys are probably the simplest design, in that participants are approached only once, at a single time point. Although advantageous in terms of low cost and ease of data collection, they can suffer from time of measurement effects, where respondents can be overly influenced by recent events (Breakwell, Hammond, \& Fife-Schaw 1995). Time series surveys are a series of cross-sectional surveys, where new respondents are approached for each wave of data collection, separated by a suitable time frame, a valuable method for assessing the impact of time. However cohort effects, differences attributed to asking a different set of people, can become confounded with time of 
measurement effects and this design can also be expensive. Longitudinal designs measure the same sample at a number of different time points, useful for monitoring developmental changes and the impact of life events, a design which reduces the problems of cohort effects. However, sample attrition, the possibility of respondents dropping out, increases and measurement effects can be confounded by age related development (Sarantakos 1998). Longitudinal studies also remove the safeguard of anonymity and respondents may become conditioned over time as to what is required of them.

Disadvantages of survey methods include sacrificing the detailed insight into the complex and contradictory ways in which people, due to their individual characteristics, think about the relevant issues, in favour of collecting large quantities of data. There is a danger that by grouping the data it can lose value. Surveys distance the researcher from the researched in acccordance with the traditional criteria of validity, reliability, objectivity and generalisability.

There is extensive discussion regarding what constitutes a good response rate. There is no absolute answer since the response depends on the topic, design and nature of the survey. Response rates for postal surveys of the general public can vary from $80 \%$ for inoffensive topics, to $40 \%$ for topics of a more sensitive nature. Surveys conducted in the format of a structured interview get greater 10-15\% response rates (Breakwell, Hammond, \& Fife-Schaw 1995). In general longer questionnaires provide lower response rates than shorter ones.

The data generated in Phase II of this study, using survey methods, provide findings that $84 \%$ of critical care units have information leaflets for relatives and only $10 \%$ of units have official policies concerning relatives. These figures which are only 
descriptive are useful but are only a small section of the research. Used in conjunction with interviews and focus groups, where relatives and staff can discuss the issues in detail, they deepen the understanding of the area under investigation and provide a more complete picture.

The survey method used in Phase II of this study, did not require sampling methods, as every adult general critical care unit listed on the Directory of Emergency/ Critical Care Units was approached. The busy nature of critical care units was taken into consideration and the survey designed to ensure that minimal effort was required on the part of the respondent. A survey was chosen for Phase II because it is a simple and straightforward way of generating large amounts of data about a population. The survey in the present context is used as a means of obtaining national data about information provisions and to determine if these provisions are meeting government guidelines. Feedback of the results from Phase II was returned to the units in the form a published article. The information gathered was used to help design the interview protocol for Phase III of the study.

\subsection{Ethical Considerations}

Ethics is the branch of philosophy that deals with morality. The problems of ethics relate to obligation, rights, duty, right and wrong, conscience, justice, choice, intention and responsibility (Burns \& Grove 2001a). Approval to conduct all phases of this study was sought and granted by the relevant research ethics committees to ensure that the rights and dignity of all respondents were protected (see Appendix A for letters of approval). The process involved submitting an ethics application to each committee, in accordance with their individual requirements (see Appendix B for one of the applications). The proposal contained information concerning the aims of the 
study, methods to be used, the potential participants and the protection of those participants and the dissemination of the results. The researcher attended the committee meetings, was interviewed by the panel and answered any questions and concerns. Specific ethical considerations or problems that arose during each phase are discussed in the relevant chapters.

One of the units involved in Phase III was due to carry out their own research into relatives' satisfaction of their unit. However, once approached they agreed that it would be more beneficial for them to allow this research to be carried out instead. The results from each phase were fed back to the units involved.

Research into critical care is considered a sensitive research topic due to the highly emotionally charged environment that relatives are exposed to. Sensitive research has been defined as studies in which there are potential consequences or implications for the participants directly, or the group of individuals represented by the research (Sieber \& Stanley 1988). Studies on sensitive topics raise questions about the nature of research that is tolerated by society and the extent to which it impacts on people's lives (Lee 1993). However, it is these very topics that often address some of society's most pressing social issues and policy questions (Sieber \& Stanley 1988). In turn, studies involving sensitive areas may aid the generation of theories because they challenge existing views of the world that have been taken for granted (Lee 1993).

When applying for ethical approval, one concern that had to be addressed was that the study would not cause relatives any further distress under in the existing difficult circumstances. Units were assured that if such a situation arose interviews would be terminated and the relatives, if they wished, would be seen by a professional member of staff, either the nurse in charge of the unit or by a Clinical Psychologist, who co- 
supervised the project. At the completion of Phase I it was discovered that relatives appreciated being given the opportunity to tell their story and the experience, far from being distressing, had the potential to be beneficial for the participants. Relatives of patients who had died or were dying were not approached.

Safeguards imposed in place by Ethics Committees to ensure the physical and emotional well-being of participants included informed consent, a guarantee of confidentiality and anonymity and the right to withdraw at any stage without justification. These safety measures have developed as a direct result of the Nuremburg Tribunals, which publicized the unethical activities of Nazi medical experiments conducted between 1933 and 1945. From the Nuremburg Code, an ethical code of conduct, was developed (Burns \& Grove 2001b) which provided the basis for the Declaration of Helsinki, adopted by the World Medical Association in 1964, which has since been amended (Burns \& Grove 2001b).

The participants in this study received verbal and written information about the purpose of the study. There were given a minimum of 24 hours to decide whether they wished to take part and participation was completely voluntary. There is a debate in qualitative research as to whether, due to the evolving process of research methodology, the participants can ever be fully informed about the focus of the study. The initial area of enquiry may be outlined but subsidiary questions may emerge and shift the focus of the research (de Raeve 1994). For Phase I, all the interviews were tape-recorded and therefore consent was obtained both at the beginning and the end of the interview (dual consent) to ensure that the participant agreed that what had been recorded could be used as part of the study. This gave participant a second opportunity to withdraw if they had said something they later regretted. This also applied to the focus groups in Phase III. In Phase II, consent was assumed if the 
critical care units returned the requested information. Relatives in Phase III were asked to sign the proforma at the completion of the interview to signify that what had been written was an accurate representation of what had been said.

Data from Phase I and III were collected via both individual and group interviews. The purpose of a research interview is to gather data; however it can be argued that any interview acts as an intervention by making participants think about thoughts, feelings and experiences (Patton 2002). Indeed, relatives in this study acknowledged that they appreciated having someone to discuss their experiences with. The researcher however, did not make judgments or offer any advice and each relative was interviewed only once, so the extent to which the interview could be deemed an intervention was extremely limited.

All participants in Phase I and all the critical care units in Phase II were guaranteed anonymity and confidentiality of the information given to the researcher. This also applied to the relatives in Phase III, however, due to the nature of focus groups, this claim could not be made for the staff in Phase III. There is current shift in thought between anonymity and confidentiality and participant's ownership of their own data. Traditionally researchers have disguised research sites and used pseudonyms for participants as a way of protecting identities which is the case throughout this thesis. The assurance of confidentiality can enhance trust and legitimatize the research process (Kelman 1972). It is also assumed that if participants cannot be identified then they cannot be harmed by publication of the research (Lee 1993). However, there exists another school of thought that believes participants are entitled to 'own their own stories' (Patton 2002). The aim is to empower participants by using real identities, for example, some politically active groups may want their voices heard. This could also protect the participants against any falsehoods made on the part of the 
researcher, accidental or otherwise (Fielding 1982). However, this may hinder research into sensitive topics and make access to certain research sites difficult. Staff that participate in the focus groups in Phase III were assured confidentiality and anonymity on the part of the researcher and in any future publications but were made aware that the nature of this research method meant that others in the group would know their identity and the researcher could not guarantee anonymity and confidentially from other participants.

\subsection{Summary}

This chapter has discussed and evaluated both qualitative and quantitative paradigms, methodologies and methods in turn. It has justified the use of different research methodologies and methods in each of the phases of this thesis. Phase I adopts grounded theory methodology and interview methods. Phase II adopts a quantitative approach using survey methods and Phase III reverts to grounded theory methodology and uses interview and focus group methods. The sensitive nature of carrying out research in the critical care environment and the ethical issues that have to be taken into account when conducting this type of research are also discussed. The following chapter discusses Phase I in detail. 
Chapter Four

PHASE I: RELATIVES' EXPERIENCES AND STAFF PERCEPTION OF RELATIVES' EXPERIENCES OF CRITICAL CARE 


\subsection{Introduction}

A review of the literature draws attention to the multitude of factors that may precipitate stress for relatives in the critical care unit. However there is little research evidence to support such views. As concerns about complete patient care increase, more attention is being paid to families of patients, in the hope that they can improve overall patient outcome and assist with the recovery process. Concurrent with recognition of family involvement, the importance of psychological well being is becoming more prominent amongst the medical profession, as such, it is important to recognise the well being of relatives as well as patients. The NHS plan (Department of Health 2000a) strengthens the rights of patients and their families by stating that "the NHS will shape its needs and services around the needs and preferences of individual patients, their families and carers" (2000b, p4).

Currently, there is little information about relatives' reactions to critical care. The most prominent article still being Molter's (1979) more than two decades ago. There is also little information about whether relatives of patients with brain injury have similar or different experiences to other relatives (Mathis 1984).

This is not a new topic, but due to its sensitive nature and seeming lack of urgency, it is one that has been neglected. Wallace (1971) suggested that it would be advantageous to employ a nurse for the specific purpose of tending to relatives. More than three decades later, this still seems like the ideal solution, but research is still tentative and practice is still struggling.

Historically we have come full circle from care in small local hospitals and extended family care to technological advancements, larger hospitals and care by strangers 
amid medical machinery. The emphasis is once again returning to community and care for the complete family unit (Hoover 1979). As a consequence, the following study looks at the experiences of relatives who have a family member in critical care. The study is split into two parts. Part A explores the experiences of relatives and Part B explores staff perceptions of relatives' experiences.

\subsection{Objectives of Phase I}

This was an exploratory study whose results will be used to inform a larger scale study. The aims of this study were threefold.

1. To identify themes and concepts in order to try to understand relatives overall experiences of the critical care environment

2. To identify what, if any, elements of the critical care experience relatives found psychological traumatising

3. To identify themes and concepts in order to try to understand how staff perceived these experiences.

\subsection{Method}

This study used qualitative methods and grounded theory methodology. For a more detailed discussion of grounded theory see Chapter 3, p 57. In this instance, the major advantage of qualitative research is that it is concerned with interpreting individuals' experiences and uncovering their meaning. These methods were chosen because of the ability to identify patterns and discover new areas. The major advantage of qualitative methods, in this case, is the ability to construct and test concepts and theories in order to generate hypothesis. 


\subsection{Recruitment, data collection and analysis}

\subsubsection{Relatives recruitment}

An adult sample of eight relatives, aged 18-65, was recruited through a brain injury rehabilitation unit (BIRU) in London and a general critical care unit from a district hospital in Surrey.

A relative was someone who was considered significant to the patient. Relatives who could not speak English were excluded from the study because there were no interpreting services available. The patient had to have survived critical care and themselves be aged 18-65 at the time of admission. The patient had to have been in critical care for 48 hours or more. The interviews with relatives recruited from the BIRU were carried out up to a year after the patient's discharge from critical care. The relatives were approached and interviewed by a Clinical Psychologist at a rehabilitation unit. There were six interviews in total. Relatives were interviewed once only. All the relatives were female.

Relatives recruited from the general critical care unit were initially approached by a member of staff, at the point of the patient's discharge. They were given an information sheet with a tear-off slip for their contact details should they decide to participate (see Appendix $\mathrm{C}$ for information sheet and consent form). The details were then passed to the researcher who contacted the relative directly.

Of the patients admitted to the general critical care unit one was admitted due to a chest infection, the other due to stomach cancer and heart problems. In both cases, the patient was a parent and the relative was a child. There were six cases where relatives refused to participate in the study, three relatives felt they were under too much stress 
to participate and three relatives agreed to be interviewed but withdrew after the death of their patient. There were two interviews in total; one male and one female. Relatives were interviewed once only.

\subsubsection{Staff recruitment}

An adult sample of critical care staff, aged, 18-65, was recruited through the general critical care unit in Surrey. All the staff were qualified critical care nurses working on the unit. The staff were given a series of talks by the researcher over a period of a fortnight to cover all shift changes in order to inform them of the details of the study. After they had received the information, anyone who volunteered was interviewed (see Appendix D for information sheet and consent form). There were five interviews in total. Each staff member was interviewed once only. All the staff were female.

\subsubsection{Data collection}

The data, for both relatives and staff, was collected through tape-recorded, semistructured, interviews. Consent was sought both at the start of the interview and after the interview was terminated (dual consent). This allowed the participants the opportunity to withdraw if they said something during the interview that they decided they did not want recorded.

A flexible interview guide was developed, including broad, opening questions and more specific follow up clarifying questions and prompts (see Appendix E for initial interview schedules). Examples were sought wherever possible. The interviews were arranged for participant convenience. Ethical considerations specified that the interviews had to be held at the hospital. All interviews were held in a seminar room on the critical care unit. Each participant was interviewed once only. In order to draw 
conclusions and verify those conclusions the interview guide was further developed to include previously raised issues and emerging concepts.

A good relationship between the interviewer and participant is essential in qualitative research. Efforts were made by the researcher to pose clear questions, be engaged and attentive and maintain eye contact. This developed rapport to encourage participants to share their experiences. The interviews lasted between 30 minutes to 1 hour. They were transcribed verbatim, excluding names and identifying features. The number of staff interviews was determined by data saturation (no new information was being added by the data collected).

\subsubsection{Data Analysis}

The transcripts were prepared and analysed as detailed in Chapter 3, p 60. A coding guide was developed to standardise the coding procedure. The coding was triangulated by two independent researchers, both with experience in qualitative research methods and methodology, thus enhancing the reliability of the themes (Mays \& Pope 1995). One of the researchers had experience in critical care nursing, thereby improving the accuracy and validity of the themes. As the interviews continued additional data gained were used to verify schema and refine the taxonomy and concepts.

\subsection{Ethical Considerations and Gate-Keeping Issues}

In order to assure the integrity of the participants the study was designed within ethical guidelines. Ethical approval was gained from North West Surrey Local Research Ethics Committee, Mid Sussex Local Research Ethics Committee and the 
University of Surrey Ethics Committee. Research and Development approval was gained from St. Peter's Research and Development committee and Brighton and Hove University Hospitals Trust Research and Development Committee.

Once all the relevant approval was gained the researcher held a series of talks and presentations, over a period of 4 weeks, for Clinical Governance Committees and members of staff on the units (see Appendix $\mathrm{F}$ for an example presentation).

For this phase of the overall study relatives were not allowed to be approached until the patient had been told that they were going to be discharged from the unit. This meant that from the outset the sample was going to have a strong positive bias. As many transfers occur suddenly due to demand for beds, this sometimes made it difficult to "catch" relatives before they left the unit. In addition, in order for ethical approval to be given it was recommended that the relatives had to be approached directly by a member of staff and not the researcher. After the initial enthusiasm of the staff at the briefings, help with the study waned and the nurses did not give out the information leaflets. It added to the already large workload of the staff and was not their top priority, meaning that lots of relatives who were suitable were not approached.

In the original design of this study, two different critical care units were to be used; a general critical care unit and a specialist neurological critical care unit, in addition to the brain injury rehabilitation unit. However when the study was due to commence the specialist neurological critical care unit withdrew.

The study's use of both relatives undergoing rehabilitation and those still going through the critical care experience was justified because it was felt that relatives, undergoing rehabilitation up to a year after the event, could still encompass the 
limitations that arise with retrospective studies, such as selective memory. Using relatives who were in the midst of the situation as well would validate previous findings. However, the difficulty in actually recruiting relatives for whom the situation was current implies that it is too stressful a time to approach people. Further studies would need to take this into consideration.

\subsection{Part A: Results and Discussion from Relatives Interviews}

\subsubsection{Description of sample}

The sample consisted of eight participants, six of whom had had a family member in critical care, due to an initial illness or injury, who then developed consequent brain injury. All admissions were unplanned. All the participants were female and all patients were attending long term rehabilitation

The interviews took the form of a discovery interview. Relatives were able to tell their story from the day that their family member went to hospital to the current time. All the patients were admitted through accident and emergency. All patients were in a critical care unit for at least two weeks. At the outset of the interview it was made clear that the interviewer would be asking about experiences including their time in critical care. After that the relatives talked about critical care reasonably unprompted.

The remaining two participants were relatives who had patients in a general critical care unit. One was male, the other female. There were no differences in the themes that developed during the analysis and so it was treated as a single sample. 


\subsubsection{Themes}

Initially the data was analysed inductively in order to establish patterns, themes and categories. To do this, the data was coded line by line through open coding. The codes were refined through axial and selective coding. From this themes and categories were formed by comparing codes for similarities and differences according to their properties and dimensions and grouping them accordingly (Strauss \& Corbin 1998). During the process of coding the data, and as more data became available through continued interviews, the titles and descriptions of concepts were refined. The later generation of hypotheses about the relationships between the concepts involved deductive processes. For a detailed discussion of this see Chapter $3, p 60$. The themes that were developed fit into four main categories (see figure 8);

- Relatives

- Patient care

- Information

- Unit procedures

These categories are comprised of various related concepts e.g. the 'relatives' category is made up of concepts such as 'knowledge and learning' and 'emotional reactions'. Each category and its relating concepts are discussed in detail below. 


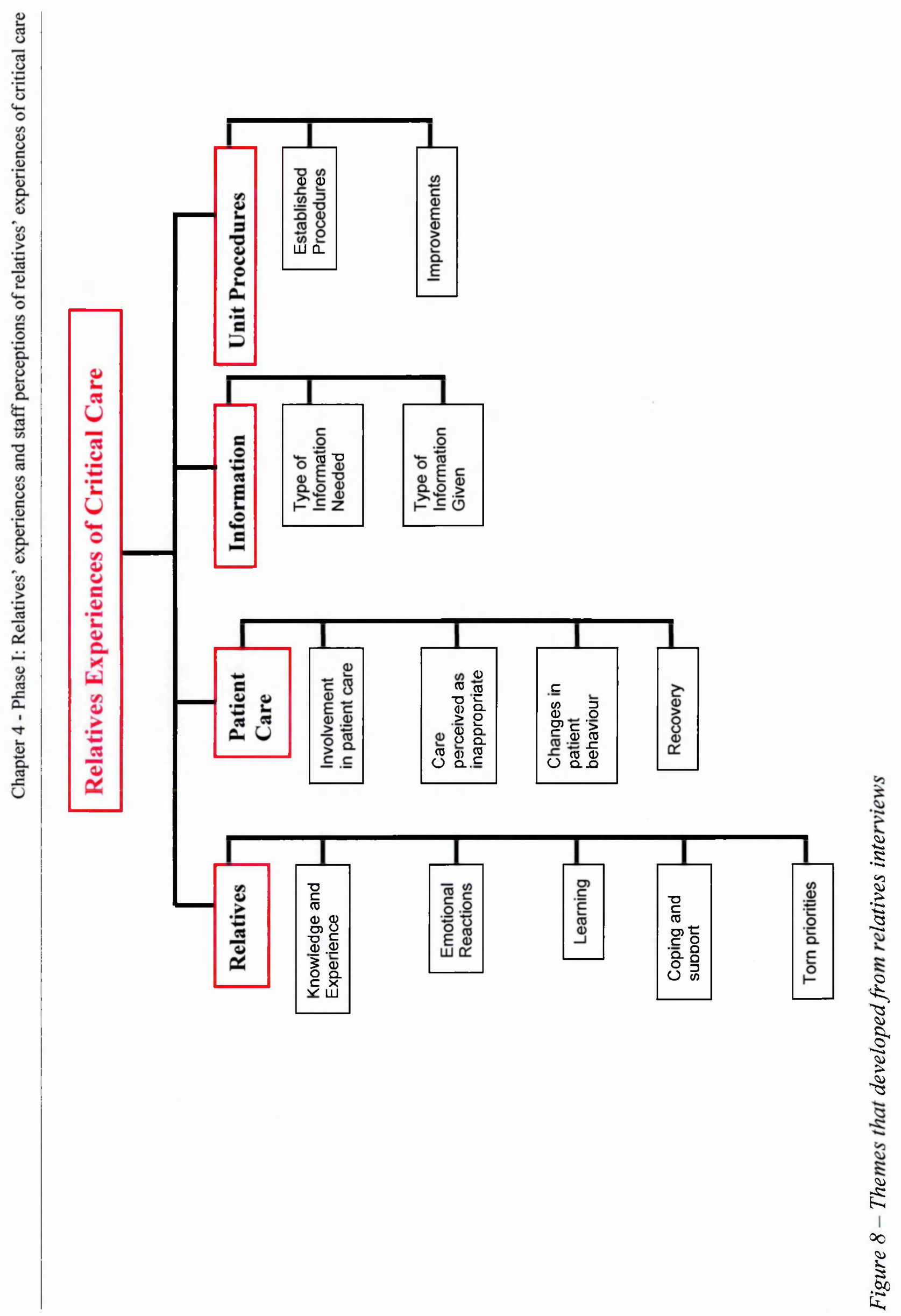




\section{Relatives}

The themes that emerged under the 'relatives' category fell into one of five concepts; knowledge and experience, emotional reactions, learning, coping and support and torn priorities.

\section{Knowledge and Experience}

Knowledge and experience relates to people's preconceived ideas about hospitals and critical care and the influence of any previous hospital experiences. The admissions in this sample were all unplanned. Therefore the relatives were not psychologically prepared for the event. However there is no empirical evidence to suggest that planned admissions do actually cope better. Many preconceived ideas are due to extensive media coverage. Relatives felt that television programmes prepared them for the experience,

“...I think that nowadays with television you see so much of it on the drama documentaries you get a picture of what you are going to see but the difference is it is your husband that is lying in the bed..." (relative 1, female, brain injured patient).

“...I think TV does, plays a big part, you know what to expect, I think these days..." (relative 8, female, general critical care unit).

However, some realise that television is not always an accurate source of information and the reality can be a shock,

“...people don't wake up like they wake up on telly. I was watching Phil Mitchell the other week when he was in a coma and when he wakes up he is absolutely normal. It doesn't happen like that but you 
think it does when you haven't had any experience..." (relative 5, female, brain injured patient).

It would seem that the media prepares people in an unrealistic way. Media portrayals seemed to affect people's views considerably, leading to the belief that critical care units are frenetic places and that there are more doctors on a critical care unit than in reality.

“...I have never been in intensive care before, but I think if you had asked me prior to this what was it like, I think I would have thought people running around like blue-arsed flies..." (relative 7 , male, general critical care unit))

Similar results were found in a study on cardiac patients (Wiles 1998), where people who suffered a heart attack thought that the attack itself would be much more dramatic as their perceptions of heart attacks from the media were people unconscious or clutching their chests. Although beneficial to a certain extent, the media portrayal of hospitals and critical care units may be detrimental to relatives coping abilities if the relative is unrealistically prepared.

Themes also emerged with regard to previous hospital experience and prior knowledge of medicine and the surroundings. One relative perceived that because her father had been in critical care previously, she knew the routine and did not need extensive explanations therefore the environment was less of a shock. Knowledge of the medical profession is obviously an advantage. One relative, who worked as a nurse at another hospital felt that she was treated better than an 'average relative' due to her profession, 
“...I think they probably did a little bit more because I was a nurse though it is horrible to say it..." (relative 2, brain injured patient, female)

She commented that she was allowed to do things that other relatives would not have been. For example, she was allowed to go in an ambulance when it would not have normally been permitted and visit during patient quiet times. People who already know the environment have a better understanding and therefore may employ more successful coping strategies.

\section{Emotional Reactions}

A vast array of emotional reactions emerged. These included emotions from fear and shock to frustration and guilt (see Table 3 for extensive range). Relatives from the general unit did not get the feelings of uncertainty because they knew that their patient was going to recover.

Table 3 - Range of emotional reactions relatives experience in critical care

\begin{tabular}{|l|c|}
\hline Range of emotions relatives experience & Incidence from group of 8 \\
\hline Fear & 3 \\
Shock & 4 \\
Confusion & 4 \\
Worry/Anxiety & 5 \\
Irritability/Anger & 1 \\
Guilt & 3 \\
Numbness & 6 \\
Depression & 2 \\
Uncertainty & 2 \\
Abandonment & 1 \\
Frustration & 1 \\
Denial & 2 \\
\hline
\end{tabular}


Initially, relatives seemed to experience reactions of complete shock and fear, which seemed to differ from later reactions of worry,

“...I was just in a daze, in shock and I was thinking I can't deal with

it..." (relative 2, female brain injury, patient)

“...my initial experience as complete shock...I've sort of been in a state of panic and being very stressed for the first two or three days, I have got to the stage now, I don't mean to sound callous, but I'm coping but I'm almost numb...I suppose it is a sort of depression or whatever when I keep coming in and there is no obvious improvement..." (relative ?, male, general critical care unit)

Relatives also suffered from transfer anxiety. There was a general fear of change, regardless of what the change was, for example, transfer to a different ward, or altering the drugs a patient was given. The relatives in from the BIRU were exposed to critical care for extended periods of time and most got to a stage where they felt that as long as the patient was in a coma he was alive, but if the medication was altered or ventilation removed he may die. Relatives had developed a routine and when that routine was altered they felt like they were starting again,

“...it was a new intensive care unit and a new relative's room to sit in and so that was a big ordeal because you feel comfortable with what you know..." (relative 1, female, brain injury patient)

There was an 'emotional rollercoaster' among the relatives of brain injured patients as the patients improved from the original condition and then regressed due to the brain injury. 
Relatives from the BIRU experienced feelings of uncertainty and abandonment that did not emerge in relatives from the general unit. This could be linked to the nature of the illness. Relatives from the general unit had not yet gone through a long and painful rehabilitation process. These feelings may have been associated with the relief that the patient was going to be okay and the worry that they may not be the same person they used to be.

\section{Learning}

This evoked themes around getting to know the critical care environment and learning about the patient's condition. It also encompassed understanding the equipment and learning to cope. This, as with many other aspects, is strongly associated with communication between the staff and the relatives. Relatives struggle with knowing how to behave in such a strange situation,

“...I didn't know what to do really. It was very strange. I held [his] hands and thought what do you do now. It was a very strange situation. I thought there was no point talking to him because he is out of it. I just stood there for a few minutes and took it all in all the surroundings and looked at the monitors. You are faced with all this television screens with all these bleeping lines so I was asking questions really on what they all meant and I didn't stay long at all....you don't feel productive..." (relative 1, female, brain injured patient)

Relatives tended to feel lost, useless and in the way when their patient was in critical care. The nurses in Part B seemed to perceive this very accurately. One nurse commented that it was almost like the relatives had to "relearn touch". Relatives felt that they did not know how to act and what do, 
“... I was expecting her to be very ill, I didn't expect her to be completely unresponsive and I found it quite awkward when I go to sit, sat with her particularly the first few days. I didn't know if I should be talking to her, I didn't feel comfortable talking to her, but perhaps that's just me..." (relative 7, male, general critical care unit)

Relatives admit that they clung onto what hope they could find. Hope influences survival and is essential for effective, healthy coping according to much of the literature (Miller 1991). It also plays a considerable part in physical and emotional well being (Patel 1996). In this relative's case, it was monitoring one aspect of the patient's condition,

“...I remember he had a pressure thing in his head that was yery, very high and that is what dominated the whole of his stay...in intensive care, looking at that pressure level. It had to be below a certain number. I can't remember what that number was, 5 or something and his number was thirty. I remember just looking at that all the time..." (relative 3, female, brain injured patient)

In Part B the staff perceive this very accurately. The staff discuss how relatives absorb very little information and cling onto one thing that they understood. This is also supported by the literature discussing the inability to absorb complex information in a crisis (Rosenzweig, Leiman, \& Breedlove 1996).

Getting to know the nurses was important for the relatives. It seemed that staff were perceived as being more caring and supportive if they took the time to get to know the relative a little bit and not just showing concern for the patient. 
“...we just started talking [the relative and a staff nurse], but you know, it wasn't just about mother, it was general and that was quite nice..." (relative 7, male, general critical care unit)

One relative commented about a particular staff member, after the tape had been stopped, that it was very comforting how she wasn't afraid to give you a hug and as a relative she had really appreciated that. A nurse's approach is a very individual thing. A relatives experience will be greatly affected for better or worse depending on the nurse they come into contact with.

\section{Coping and Support}

This concerned getting used to the patient's condition and learning to deal with it, getting to know the critical care unit and feeling more comfortable with it. Relatives appeared to get support from three main avenues; nursing staff, family and friends and/or other external organisations. Many felt that they would have like to have known about other organisations sooner and it would have taken the pressure off the family unit.

“...I would never have known that here was available. You have a patient with neurological problems who are sent home it is like there is a no hope situation. You know what I mean. Like they are going to be like that and there is nothing you can do about it and they will have to go home and be cared for by who ever is there. But you realise that there is more available. There are other organisations to help. It is not a dead end situation. I think that has really helped..." (relative 3, female, brain injured patient) 
This would correspond with the literature that suggests that the main area for social support during this type of crisis would be family and nursing staff (Woolley 1990). Relatives recruited from the rehabilitation unit placed importance on external organisations and appreciated the help and support gained from them. This did not emerge with the relatives recruited from the general unit. This may have been because those relatives had not thought that far ahead yet, and the main priority was getting their loved one out of critical care.

In the interviews that were conducted from the general critical care unit the relatives were referred by one particular nurse, who was experienced and obviously very good at her job. The people who agreed to be interviewed had coped well with the experience. It was the relatives who refused to take part who were having problems coping and the ones who had been most affected by the experience of having a relative in critical care.

\section{Torn Priorities}

Relatives often find that they have torn priorities when a family member is in critical care. This can lead to emotions such as guilt and can be a factor in increasing stress and anxiety levels.

“...I asked them to transfer him...because my daughter was getting too, it was taking too much out on her. She was getting very emotional. I think she thought I was never going to come home again and she needed me. So I needed him to be closer to home so that I could be there for her..." (relative 2, female, brain injury patient). 
People's lives have been severely disrupted and it is very difficult to regain that equilibrium.

"...The issue for me is that she is pretty out of it, does she know I'm there, well no she probably doesn't and although I feel a responsibility and want to be here I also have a family. I've also got a job. And I am really getting to that stage where I have to try and start to balance those...I feel guilty when I leave here to go home and I feel very guilty when I come back up because...I don't know..." (relative 7, male, general critical care unit) [The gentleman lived considerable distance from the hospital.]

\section{Patient Care}

The themes that emerged from the patient care category were divided into four concepts; involvement in patient care, inappropriate care, changes in patient behaviour and recovery. Relatives from the general critical care unit commented that they could not fault the care that their family member received and generally they were satisfied with their experience. The care their patient received during their stay has been praised and any grievances appear to be with another part of the 'illness story', e.g., the problems of being transferred to a general ward.

Relatives from the general critical care unit did not discuss the staffing issues and they had not yet been through the recovery process, so they did not have that experience to discuss. In addition, because their patients had more general complaints the hospital were able to deal with them adequately, so issues surrounding inappropriate care did not arise. A theme that both samples had, common under this category, was involvement in patient care. 


\section{Involvement in Patient Care}

Literature suggests that having relatives involved in patient care can assist patient recovery (Dyer 1991). Relatives indicated that they would like to be more involved in basic patient care and more informed of patient progress. The relative in this case was accidentally allowed to attend her patient's ward round because she was a nurse at another hospital. Commenting on relatives not being allowed to attend:

“...which I think is wrong. Because you know, nothing should be said that can't be said in front of the patient anyhow, just because the patient is in bed. And I've always thought that relatives should be able to attend ward rounds. Not necessarily to be a part but to ask questions at the end. I don't necessarily think they should be there but if they are there it shouldn't be a problem..." (relative 2, female, brain injury relative)

It seems that relatives want to be involved in patient care and staff agree that relatives could be an asset, yet they are still not given the opportunities,

“...both my sister and I helped to wash him or clean his teeth...we didn't ask, we just got on and did it. We just said to dad, do you want us to freshen you up and he said yes and so we did it ourselves..." (relative 8, female, general critical care unit)

\section{Care perceived as Inappropriate}

This seemed to be a recurring theme because brain injury was not the patient's initial complaint. The patients were admitted to general hospitals which were perceived by relatives not to be equipped to provide the specialist care required, 
“... I kept thinking about brain damage and injury. That kept coming up in my mind and thinking, is he going to have damage to the brain. And they said well we can't tell you that because the equipment that we need to tell you that we haven't got here..." (relative 1, female, brain injured patient)

An additional problem was that the patients were often placed on wards that were perceived as inappropriate by relatives. Many of these problems seemed to arise because brain injury is not picked up instantly and there were other more immediate complications that needed dealing with,

“...It was difficult because as it was a cardiac ward they focussed very much on his heart and obviously that was a worry but I did focus more neurologically...It was quite difficult because there were middle aged men there...I just thought it wasn't the right place for him..." (relative 2, female, brain injury patient)

“...Orthopaedic...But it was all old people. And that was quite difficult because I feel [he] needed to go somewhere for head injuries because he was very strong willed and very wandering around talking to people and trying to help with their medication. And helping the nurses with washing them. I think they found him very hard work..." (relative 3, female, , brain injured patient)

In addition to this some relatives had concerns about members of staff. This is another issue that is very individual to people and establishments,

“...there were little things like his management that I wasn't happy about. I don't know much about intensive care or medicine but there 
were little things like for example nursing issues. There was one time when he was sweating literally buckets... and the nurse who was looking after him said that I'm not going to bath him now, I'm going to do it in an hours time...Just silly little things like that and there was another time when he was being weaned off the ventilator and he was really, really struggling and everyone was looking at the machine the blood sats but nobody was looking at him. I was glad I was there to say something so that something could be done about it but I didn't think it was right that I had to be there to say something..." (relative 4, female, brain injured patient)

“...we had one very uninterested nurse who was just terrible really. I don't know if she was trained but she was young and there was no feeling of confidence so it all went..." (relative 6 , female, brain injured patient)

\section{Changes in patient behaviour}

Brain injury can cause a patient to act or respond in an inappropriate fashion. These changes in behaviour can be particularly distressing for relatives because their loved one appears unrecognisable. Injury to the brain can cause problems not only in behaviour, but in emotions and cognition.

“...one day he was quite, he understood what you were saying to him.

He could blink his eyes and that and my brother went in to see him that evening and my brother had made him a board with different symbols on that he could point to, which was brilliant because this was the first day in a week and a half - two weeks that we had had anything out of 
him...but the next day when I went in to see him he was, it was so weird because he had just regressed. Because they had had to put his mattress on the floor because he was just thrashing about and he didn't know who I was at that stage. He wasn't communicative. He was spiteful...the regression frightened me so very much that I asked to see the surgeon..." (relative 3, female, brain injured patient)

“...but of course he didn't have a clue what he was doing and he had thrown himself on top of another patient at which point they thought they had better move him into his own room and get him a one to one person. They got him a one to one person and he still managed to break his nose at that time but I don't think it was ever recorded. He was hurling himself around. And they did not want to sedate him because they wanted to see how he was. I was there quite a lot of the day and he slowly became more controllable but still quite badly behaved..." (relative 6, female, brain injured patient)

The fluctuation in condition and deterioration to an dependent state that occurred in the patients was the most distressing aspect for relatives. Many felt that their patient could have been better cared for during this time and that the dignity of the patient could have been preserved better. They attributed the deficits in care to patients being on inappropriate wards where staff were simply unable and unequipped to deal with the patient properly. 
Recovery

The recovery process takes a long time, but recovery from brain injury takes even longer. Although relatives experienced relief once they knew that they patient was going to be fine, it is a very slow process,

"...when I went in to see him the following day and they had woken him up and I remember walking into intensive care and looking across and him looking at me and our eyes connected and I just knew that he was fine..." (relative 1, female, brain injured patient)

Whereas, the majority of patients just have physically impairments to deal with while recovering, patients with brain injury often have mental impairments and this can put added strain on relatives,

“...but he is doing a lot more now. I still have to leave him jobs but he tells me what he's at...so he has some more insight now to how things are. He is pretty good actually. He is a little bit introverted I think...it is not a great worry for me though. His confidence is quite low, he doesn't see people so much now. But I'm sure it will improve a bit more. He doesn't watch so much TV because I leave him enough things to do. There are still a few problems but it seems to get a bit easier. When I'm tired I get annoyed with him for not remembering something but it is not his fault. He is very good..." (relative 2, female, brain injured patient)

\section{Information}

The themes that emerged from the 'information' category were divided into two concepts; type of information needed and type of information given. 


\section{Type of information needed}

This includes assessing and deciding which information is needed at what time. It includes what relatives feel they need and the individual needs of every relative, “...I mean I am naturally inquisitive as a person, thinking what's this doing, trying to work out where the leads are going and stuff. No they didn't [explain what the machines meant] but that doesn't, I think it is always difficult really. I'm just a bit of a worrier, and I think had, it's a fine line, because I think, had someone sat down and said this it this and this and what it really means, probably that would have alarmed me more..." (relative 7, male, general critical care unit)

“...possibly better communication. I do think the doctors weren't particularly helpful. The nurses were fantastic, very supportive. But I didn't see that much of the doctors and I do feel they do not give enough information really. But I was always under the impression that they were busy. I know they are but you know it was quite a serious thing and [he] was very young and so I just thought that they could have been a bit more supportive really. It didn't really bother me at the time really because the nurses were so helpful, but it wasn't a particularly good thing..." (relative 2, female, brain injured patient)

Relatives comment that they did not have much contact with doctors. It could be that they had the standard amount of contact with doctors, but as a nurse in Part B points out, television portrayals of hospitals give the impression that they are more hectic and that doctors are always present. One relative explained that in the course of 10 days he saw the doctor twice, 
“...I have had fairly limited contact with doctors. When she was first admitted I sat down with a consultant... who painted very realistic picture of her chances of getting out of it. I had limited contact with the doctor up until yesterday when I sat down and had a discussion about the operation..." (relative 7, male, general critical care unit)

This also coincides with the view that often relatives expect doctors to be around all the time due to TV portrayals as emerged in the knowledge and experience theme. Relatives need consistent information and this seems to be one of the hardest things to get,

"...But the one thing that I wish was that you saw maybe one doctor and you had one source of information rather than two or three people with conflicting views or looking at it from different points of views..." (relative 1, female, brain injured patient)

It is interesting that in a predominantly nurse-led environment, relatives still have a belief that a doctor should give them information. The staff acknowledged that there is a lack of continuity but also discuss how hard it is to achieve the continuity that is required.

\section{Type of Information given}

This is the practical information such as visiting hours and facilities available for the relatives. It also includes information about the patient and their condition. There appears to be a lack of communication between staff and relatives, as illustrated by the following relative have to ask if her husband was in a coma, 
"...and then I said to the nurse why won't he wake up. Is he being sedated? And she said no no, he is not being sedated and so I thought why won't he wake up then and then everyone just looked at me and then it just dawning on me are you telling me he is in a coma then. And that was well, yeah..." (relative 1, female, brain injured patient)

The literature suggests that in times of crisis people do not have the emotional capacity to seek information. The information should be brought to them (Bowman 2000). This is not an example of best practice. Information of this kind should have been volunteered. Another relative commented that he was not prepared for the sight of his mother and felt that it would have been easier if they had been told what to expect and why,

“...If I hadn't got the confidence I would probably be asking more questions...One thing that alarms, well, not alarms, worries me and worries other people that come in, her sister and her best friend, she was very, very puffy and I think we had to ask what caused it was the fluid balance and blood pressure and stuff. And that, had we not been told what had happened it is quite alarming seeing someone that changed in a way..." (relative 7, male, general critical care unit)

The way in which information is given is also important, the following relative noted the distinct lack of a compassionate approach of one doctor,

"...I mean one doctor had told me one thing and then another doctor took me into a room and gave me a really bleak outlook and literally wiped the floor with me. He literally shattered me with the things that he said..." (relative 1, female, brain injured patient) 
Later in the interview she commented that once she sat down and thought about what the doctor had said she realised that it was nothing that she did not already know, but the manner in which he delivered it made her feel that there was no hope.

Relatives also felt that they were not kept informed of when and where their relative will be transferred,

“...I was furious that no one had actually called me in to be there when he was moved or rung me to say that he is no longer in intensive care he is in ward six instead of me sort of turning up. So I was sort of cross about that. I wanted to make sure that his belongings had gone because half his belongings got left behind in the intensive care unit..." (relative 1, female, brain injured patient)

One relative commented that although they were always told that their patient was being moved, for information about their patient's condition they always had to ask. This again, does not correspond with the crisis literature previously mentioned. The consistency of information varied not just between doctors but also between wards,

"...I think, again when you are in the intensive care you tend to get more information because you have got this one to one care. You've got somebody that knows everything that is going on. Once we went to the ward the nurses were different all the time and you really didn't find out much from then and then it was just a question of when the team came round, getting the information from [him] what they had said or if I happen to be there when the doctors arrive then I could sit in on it. But I suppose the information tends to dry up when you are on a normal hospital ward..." (relative 1, female, brain injured patient) 
This is a retrospective view of someone who has been through critical care and is now in rehabilitation. Compared to relatives still in critical care they think the information they received was good, in contrast with what they have had to endure since. Relatives felt that the only reason they repeatedly asked different nurses different questions was because the information they received was inconsistent.

“..and some of it [the information], I have to say, you might be told one thing from one and something else from the other one. So if we weren't happy we just asked to see a doctor. We made an appointment and saw a doctor..." (relative 8, female, general critical care unit)

However, again it appears a very individual approach, as other relatives felt they were given all the information they needed and wanted,

“...they told me up front what was happening and they were really good. I thought they were really good the way they were..." (relative 2, , female, brain injured patient)

\section{Unit Procedures}

Themes that emerged were divided into two categories; established procedures and improvements.

\section{Established Procedures}

Established procedures are procedures already in place such as opening/visiting hours, information sheets to be handed out, discharge procedures, return visits for patients to aid recovery and the 24 hour phone line. Some relatives felt unhappy about the restrictions placed on visiting hours because they just wanted to be with their patient. 
A relative felt uncomfortable when he visited his mother during the patient quiet time because he hadn't realised that there were visiting hours.

“...I've not really been told, it's on the leaflet that the give you, patient quiet time 12-2.30. The first day that she was in, I came in at 12 and I wasn't told that I couldn't see her, I went to see her, but I did feel that, you know, I shouldn't really be there. But apart from that whenever I have come in I have been allowed to see her..." (relative 7, male, general critical care unit)

Relatives agreed that the restrictions were in place for a good reason and were happy about complying with them,

“...we tried very hard to just have 2 of us at the bed, like they insist on, which I think is excellent, because the patient needs all the rest and care he can get or she can get..." (relative 8, female, general critical care unit)

"...they had a doctors round between 9.00 and 11.00 so if the doctors were doing their rounds you couldn't go in and then they had a rest period from about 2.00 to 4.00 and you couldn't go in then either. That just use to annoy me because I didn't want to be thrown out. I wanted to sit with him all the time. But at the same time it was very draining..." (relative 2, female, brain injured patient)

\section{Improvements}

Relatives felt that the move from critical care to a ward was too great and advocate the use of intermediate stages, such as High Dependency Units (HDUs). Although these do exist for the relatives in this study they appear not to have been used. 
“...I suppose an intermediate stage. I know that when he was over in the...hospital they had high dependency beds within the intensive care unit so it wasn't one to one it was maybe one nurse to two or three so there was a transition period...I think that would have been a good stage to have gone through. You know you have one to one and everything is geared around you and the bed's all singing and dancing and does all the tricks and the next thing you are in a hospital bed...and the staff are obviously here there and everywhere doing things and you know he wasn't mobile at all and that really was a more difficult stage..." (relative 1, female, brain injured patient)

One relative felt that it would have been more reassuring had they been able to talk to a trained medical professional on the telephone, rather than a receptionist. Given resources available this is not really a viable option.

“...I understand why. They can't tell you but it would have been nice if they could have spoken to the doctor at the time and found out a little bit. They don't have to say much. We do have a policy not to talk to relatives on the phone but you have to say something don't you. You are put through to a receptionist who is a receptionist. But I would have liked to have talked to a doctor or nurse. I knew he had had a cardiac arrest but I wanted to know if he was stable really. I don't think that was done very well. But apart from that they were all very nice..." (relative 2, female, brain injured patient) 
The issues emerging are focused around consistency and the process by which relatives gain information, as opposed to whether or not they are getting any information at all.

\subsection{Part B: Results and Discussion from Staff Interviews}

\subsubsection{Description of sample}

The sample consisted of five participants who were qualified critical care nursing staff. All the staff were female.

\subsubsection{Themes}

Initially the data was analysed inductively in order to establish patterns, themes and categories. To do this, the data was coded line by line through open coding. The codes were refined through axial and selective coding. From this themes and categories were formed by comparing codes for similarities and differences according to their properties and dimensions and grouping them accordingly (Strauss \& Corbin 1998). During the process of coding the data, and as more data became available through continued interviews, the titles and descriptions of concepts were refined. The later generation of hypotheses about the relationships between the concepts involved deductive processes. For a detailed discussion of this see Chapter 3, p 60. The themes that were developed fit into five main categories (see figure 9);

- Relatives

- Staff

- Information 
- Unit procedures

- Patient care

These categories are comprised of various related concepts e.g. the 'relatives' category is made up of concepts such as 'knowledge and learning' and 'emotional reactions'. Each category and its relating concepts are discussed in detail below.

Most of the categories in Part B share similar characteristics with those in Part A. The main difference is the inclusion of a 'staff' category. Under 'information' the addition of an 'importance of information' theme has been added and under 'Unit procedures' the addition of a 'system problems' category has been added. 'Patient care' emerged as a much smaller category than previously. All these differences are discussed below. 


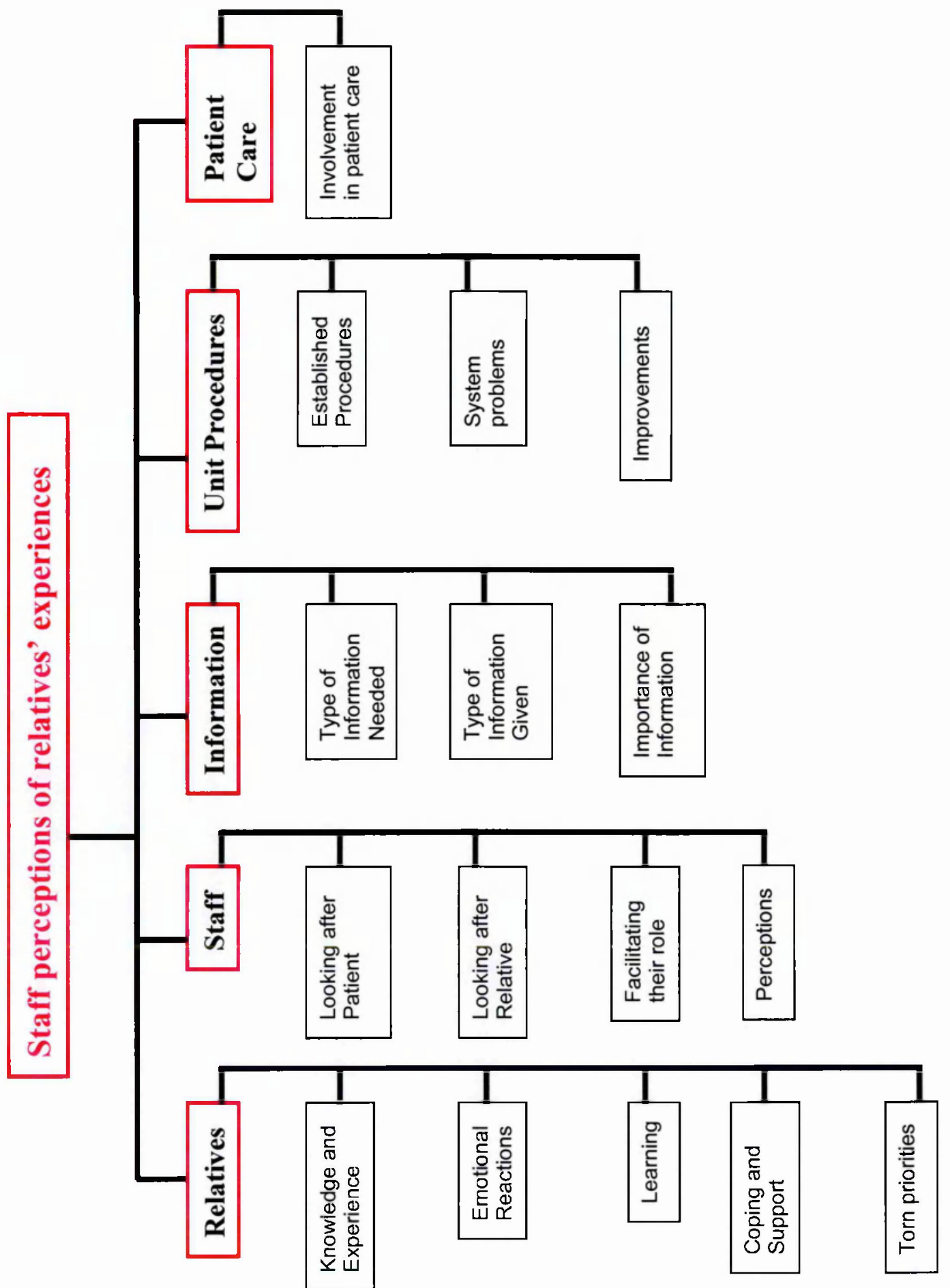




\section{Relatives}

The themes that emerged under the 'relatives' category fell into one of five concepts; knowledge and experience, emotional reactions, learning, coping and support and torn priorities.

\section{Knowledge and Experience}

This is the same concept as emerged in Part A. Relatives agreed that they felt the media prepared them for what they faced, although some acknowledged that this was not always accurate. Staff held the view that television gave relatives unrealistic expectations,

"...The interesting thing is that television has warped their views quite

a bit I think. It's not ER in our district hospitals. It will never be like

it...[the relatives think] where's all the doctors all the time..." (nurse 5)

Staff also observed that relatives of people who had been in hospital and relatives of long term illness patients cope better because it is almost expected that the patient will one day end up in critical care and they are more prepared. In addition, staff who had also been in the relative's position and likewise relatives who worked in the medical profession agreed that their profession helped them cope. They also thought that they were treated better than they would have been had they been an 'ordinary' relative. Relatives in Part A also recognised this.

"...they were very, very cool with us and they told us exactly what was going on because at the end of the day we worked, we were all colleagues and yeah, I think it made life much easier, perhaps we had a better understanding as well..." (nurse 3) 
Previous experience of having a patient in critical care seems to have dual implications. It can lead a relative to be more prepared and better able to cope. Staff also perceive that it can have exactly the opposite effect. There was no evidence to indicate that relatives agreed with this. However that could be linked to the small sample size,

"...I think if they have had the experience of having somebody sick, either long term, either in hospital or having been in critical care, the prospect of going back there must be horrific. So I wouldn't have thought that initially they would cope with that very well. Their first experience back into the critical care setting would be very traumatic..." (nurse 4)

\section{Emotional Reactions}

Staff perceive the range of emotions relatives experience quite accurately. The first 24-48 hours appeared to be the critical period during which relatives absorbed very little and emotions were at the forefront,

“...I think first there is initial fear. Then total uncontrol..." (nurse 2)

“...I think it is...an alien environment really...they walk in and are absolutely terrified, because they don't know what they are going to see despite you explaining what tubes and bits and bobs they have before they come in..." (nurse 3) 


\section{Learning}

This encompasses the same themes as the previous 'learning' category. Staff discussed how they aid relatives to learn about and settle in to their new environment, “...I do think that an explanation before they come in [enter the unit] is quite important. And I do think that if we don't explain to them what they are going to see then its just such a shock really..." (nurse 3)

However, although the benefits are acknowledged this explanation is not always forthcoming as illustrated in Part A, when relatives discussed that sometimes there were things that they would have liked to have had explained to them. Staff also recognised the overwhelming nature of the unit to outsiders. The physical environment takes a lot of getting used to,

“...It is an invasive environment. You've got procedures, you've got monitors...it looks like that body has been taken over...If you have got a patient with multi organ failure, they will have a ventilator, a haemofiltration machine, will have multiple drugs on the side and the other side, and you'll have alarms and it looks like it is dangerous to approach the patient. That you might do something wrong. The relative will say can I do this or can I do that and that's, I find that very upsetting. Of course they can it is their relative, their patient..." (nurse 5)

Again staff seemed to perceive this accurately,

"...I would say that the fact that they are frightened to touch their relative. That their relative doesn't look anything like they did before... the first thing they want to do is give them a big hug and they daren't 
even touch. And then you will often find that, from then on in it will be weeks before they dare touch them. I'll say 'it's ok you can touch them' but they can't they are frightened to touch them. And they end up through that first few hour's experience really frightened and have to almost relearn contact. I think that is where the men suffer much, much more. That the women are more tactile and will go in eventually, even if they have been put off initially, they will go in but men will find it very, very difficult..." (nurse 4)

\section{Coping and Support}

This category has the same meaning as in Part A. Coping is a very individual process, some people will cope well and others will not and this will often depend on the support they have available to them. Support can buffer negative effects or it can minimise those effects to aid relatives coping abilities. Staff discuss many avenues of support that they felt relatives used or needed to use,

“...I think if they [relatives] build up a rapport fairly early on with a nurse it does make it easier. I think we have a few problems at the moment with lack of communication and lack of rapport being built and I am sure it does help with relatives if they do feel comfortable with a particular nurse..." (nurse 1)

Strong support networks and strong beliefs (e.g. a strong faith) ease the process because the relative has somewhere to turn to. There is extensive literature that supports the view that support acts as a buffer against stress (Cohen \& Wills 1985). This is recognised by staff, 
“...Other family members. That they have a support network at home. I think helps them here. When they are in here, not that they lean on us but they ask us lots of questions..." (nurse 2)

“...They cry more [relatives with strong religious beliefs]. They have more hope. Their religion becomes their hope. They are depending on god to change something. Sounds silly but its true..." (nurse 5)

Staff also highlighted life experience, gender differences and education as factors that affect how someone copes,

"...I think it is very variable on their experience in life anyway. Some people will cope with it very well and some people wouldn't cope with it very well at all...I think the male species cope with it less well but you would be less likely to know... a woman would cry and be upset and want support and a man would be very stiff upper lip and quiet but inside would be really torn apart by what was happening within the critical care environment and the stress of having a sick relative. And sometimes the routine of having to come to hospital twice a day, I think that women are far more able to cope with a stressful routine than men..." (nurse 4)

"...level of education, sometimes, it comes down to that really. If they've experience with comprehending things..." (nurse 5)

Relatives discussed external support they received as a means for helping them cope with the situation for example other family members. In addition, staff also discussed other more internal factors for example, a strong faith, that affect how well they think a relative is able to cope. 


\section{Torn Priorities}

Staff recognised that relatives often have torn priorities when a family member is in critical care. They acknowledge how difficult it can be to balance existing responsibilities and commitments, to the new responsibilities relatives experience towards the critically ill family member. This can lead to feelings of guilt and staff perceive how difficult an emotion such as guilt can be to deal with,

“...they feel guilty when they are not here because they feel they should be, but when they are here they feel useless. And I perceive that the women are better at the sitting round in the waiting room, sitting round in the unit, just being there..."(nurse 4)

\section{Staff}

The themes that emerged under the 'staff' category fell into one of four concepts; looking after the patient, looking after the relatives, facilitating their role and perceptions.

\section{Looking after the Patient}

This concept illustrated carrying out nursing duties for the patient without letting relatives get in the way. Staff had very individual perceptions as to whether relatives were a hindrance or help in patient care.

"...There is a move towards trying to get them [relatives] to perhaps come later. The early shifts... are a busy shift you have got your doctors round, you've got your physios, you've got the fact that a lot happens on the morning shift and you got a relative that comes in, in the middle of 
it. It really does delay you. For example, what I had today was that I had some injections to give, where I don't particularly want to do it in front of the relatives. a) because you have got the risk of them feeling a bit sort of squeamish with you doing it, you are interrupting them, so you then delay it. Obviously when you have life threatening things that need doing they get done. I was giving vitamin injections, it wasn't desperately important that I did it there and then. But I think they do hinder you to some extent..." (nurse 1)

Staff have a constant dilemma between whether the patient or the relative is a priority. Ultimately the main task of critical care units is to care for the patient, but it is important not to make the relatives feel alienated.

\section{Looking after the Relatives}

This concept includes assessing relatives' needs such as, which information they need/do not need, want/do not want and giving that information. Looking after relatives also includes listening to relatives and facilitating relatives coping and building a rapport with those relatives.

"...their perception of illness and...their understanding, their willingness to understand as well as their non-willingness to understand. Some relatives don't want to know the whole story, they never want to know. Because that is their comfort zone. You have to find their comfort zone..." (nurse 5) 
The fact that all relatives are so individual makes it very difficult for staff to judge exactly what each one needs. This is probably something that can only develop with experience.

Previous research has illustrated that in a crisis people do not have the spare emotional capacity to seek support, the support must come to them (Wilkinson 1995). Bowman (2000) advocates that in a crisis situation, relatives may have severe difficulty processing and retaining information because as a person's level of arousal increases, the ability to process complex information decreases (Rosenzweig, Leiman, \& Breedlove 1996)

"... [The relatives are] looking for someone to say one thing has improved, they are looking for the positive and they hold on to the positive and they forget about the negative and it is usually an overwhelming negative and they forget about that and they cling on to the positive. Or they cling to one particular thing that they can understand and then focus on that and that is their main focus. So like it could be infection, and they understand infection, so they'll hold onto infection and keep asking about that...depending on how sick the patient is, sometimes they don't understand how sick they are. They just totally block out...You just tell them again, you have to keep telling them..." (nurse 2)

The fact that relatives cling so firmly to one aspect may be a hindrance to communication. Staff may end up feeling that if they have not got anything positive to say to the relative then it is better to say nothing, which is not the case. 
“...I don't know, perhaps it is just me, but I normally sort of when they come in I sort of sit down and have a 5 minute chat with them and say sort of how are you doing? Because sometimes you find that on the other side they are coming in and it's very stressful coming into ITU every day and spending most of the day in here, and they are not sleeping, not eating, and I would say to them, how are you? Have you eaten today? And are you sleeping ok? And if they are not sleeping maybe suggest they go to their GP, something like that. I don't know what other people do, that's just probably me, I don't know..." (nurse 3)

Staff did not discuss whether and how difficult it was to explain things to relatives.

\section{Facilitating their Role}

This concept involves things that facilitate the role of the nurses for example, organisation of patient care, such as shifts and relevant skills. It also includes practical aspects such as having a screen so that nurses are not on constant display, this would help relatives not become too dependent and thus assist in the recovery process. It also includes information handouts and making assumptions about how to deal with relatives. It is about working around relatives but also working with them.

"...someone who has come in as an emergency, to then have to go to a ward it is a bit of a wrench. It is quite hard. We make sure that we do start stepping back a bit. It not always easy...to go from here to the ward it's very difficult. And we do try, especially now with the outreach thing that is set up and trying to be developed, to follow up on patients so that they still have, so they are not sort of forgotten, or they don't have that feeling that they are forgotten about. And we always 
encourage patients to come back, as part of their psychological healing..." (nurse 1)

“...It is very difficult because you haven't got continuity of care with the nurse at the bed space, you haven't got continuity of care with your relatives or SHO's, and you haven't got continuity of care with the consultants, so it is virtually impossible [to be consistent]... if it is a weekend...the same people are on call and the same perhaps nursing staff and it makes life so much easier, and I think it makes the relatives life a bit easier as well because they come in and at least they know somebody and they have been speaking to them perhaps on Friday and its Sunday and at least they have got 3 consecutive days of it..." (nurse 3)

Again, there is the issue of consistency. There is awareness among the staff of what 'best practice' is but there is also an acknowledgement that for whatever reason best practice does not always happen. Staff are in agreement that they should be providing relatives with initial explanations and support, but the more experienced staff are aware that not everyone does.

“...in reality not always a lot [of time to give initial support]. So it depends on the level of experience of the staff as to whether or not they can give that support in the first place and if you have just got a patient back who is critically ill then taking 20 minutes out to go and sit with the family can seem like a very long time. And again, I think that is down to experience that you have to be able to be experienced enough to say to somebody else, you look after the patient, you do these tasks 
for me, I am going to go and sit down and chat to the family, which doesn't always seem like a priority..." (nurse 4)

\section{Perceptions}

This concept outlines nurses' appraisal of the unit and of others members of staff. It also covers nurses' perceptions on how to deal with patients' relatives.

“...It's bit difficult for us to assume what we didn't know what we know now about hospitals..." (nurse 1)

Staff are aware that relatives should have the consistent information that is important to them, as illustrated in Part A, but they are aware of what a difficult thing this is to achieve,

“...I think you need to be all coming from the same area, same point of view really and as long as we are all coming from the same point then I think it makes things different, they are getting told the same thing day in day out, so there is not somebody one day saying oh you know, things are looking very, very bleak, I don't think we are doing very well and someone on the next day saying well, we are going to try this antibiotic and that should work..." (nurse 3).

"...Or sometimes, there are new doctors and they are not aware that the nurse should be there [when a relative is being told information]. They don't know that the nurse is part of the process really. That is very important..." (nurse 5) 


\section{Information}

This is the same as the previous information category.

\section{Type of Information Needed}

Nurses perceived that some relatives want to acquire as much information as possible and ask numerous questions. Others appear to want to remain 'ignorant' and only know whether the patient is better or worse,

“...Some relatives really want to almost know too much and then other relatives are not particularly, whether it is the initial shock and don't want to know..." (nurse I)

"...once they have established that their relative is in whatever situation they are in whether they are dying or not dying or getting better and I think they are more open to the nitty gritty bits of information like, where the toilet is, where the coffee machine is, these are the hours that we do, this is what we do, this is what's going on..." (nurse 1)

\section{Type of Information Given}

Although the staff advocate what is 'best practice' with regard to how and when relatives are given information, it appears that this best practice is not always implemented, for whatever reason.

“...the initial 24 hours they should be spoken to by a doctor and then every couple of days they are spoken to or when they request it..." (nurse 2) 
Relative 7 in Part A discussed how he had only seen a doctor twice in ten days. How much information relatives are told is also very dependent on the nurse they talk to. Some volunteer information, others do not. The following nurses outline the best practice,

“...what we do, is obviously introduce them to the environment, ask them if they have been into an ITU or had an experience of ITU of any description..."(nurse 1)

“...Usually I just explain what is around the bed and not to be scared of it and ignore any alarms because we have just set to go off and not to worry. And if they see us panicking that's when to panic because nothing usually goes wrong...tell them that they [the relative] can touch them [the patient], they can talk to them and that kind of relaxes them a little bit..." (nurse 2)

Another nurse admits that she waits for relatives to ask questions,

“...I don't volunteer information. I wait for them to ask questions..." (nurse 5).

Staff felt that relatives could be manipulative playing one nurse off against another. Whereas in Part A, relatives felt they did this because they had not received a suitable answer from the initial person they asked,

"...the way they ask each nurse the same question, is quite a commonality. Relatives soon recognise how junior a nurse is and how experienced a nurse is and they can vary their questions to get information and how they ask that question. They check they get the 
same answer and they can start to play one nurse off against the other..." (nurse 5)

This illustrates a difference of opinion between staff and relatives. It could also be seen to contradict the evidence that relatives cling to one bit of information. However, if relatives do not like or do not agree with a piece of information they have been given, they go and seek another answer that they do agree with. Depending on how this is interpreted it could also be used to support the view that relatives need something to cling to. The latter explanation seems more likely.

Nurses, quite rightly, feel very strongly that they should be part of the information process and they acknowledge that they can help to support the relative,

"...this is how is should be done, it usually is, we make the aim to do it. They [relatives] are not told information by a novice, usually a more experienced sister or if you are senior staff that's at the bedside and has got a really good perception of the situation. They are not told, or they should not be told on their own by a doctor, although it often happens, if the doctor is rude enough, not to acknowledge that the nurse should be there. Which can happen, but obviously shouldn't happen...or sometimes, there are new doctors and they are not aware that the nurse should be there. They don't know that the nurse is part of the process really. That is very important...the doctor, the nurse and any family members who want to be present really..." (nurse, 5) 


\section{Importance of information}

This concept refers to the role that information plays in relative's coping. Information plays an important role in easing the fear of the environment. The majority of people, whether staff, relatives or researchers seem to agree that communication of information is fundamental to the coping process.

“...I think that giving information as much as they can and as much as they want as well. Because there really is this thing where some people want to know everything and other people don't want to know a thing at all...it is easier to give information and explain it. I find it odd, I think it must be awfully frightening to come in and not know what is going on..." (nurse 1)

Staff could discuss the importance of information in an objective way. This was not a concept that concerned relatives. Their primary concern for relatives was finding out what they wanted to know with regards to their patient. The meaning behind it was not an issue.

\section{Unit Procedures}

In addition to the previous themes that emerged in this category, the theme 'system problems' also developed. This theme emerged because staff discussed the problems they faced with procedures while working on the unit. 


\section{Established Procedures}

Literature suggests that relatives have difficulty absorbing information during a crisis situation, staff realise this and information sheets are in place to aid retention of general information,

"...I think that is why our information sheets, the little things that we have got, are a good idea because that's got the very basic information in and what's not taken in by the nurse talking to you. At least you can go home and when you have had a bit of time gather your thoughts a bit...there is a 24 hour phone they have a direct line and it is always made very important, clear to them that they can phone at anytime..." (nurse 1)

\section{System Problems}

System problems are issues that arise with the current system, for example there is no set way of dealing with relatives and it is a process that is very individual to the members of staff,

“...It can cause problems when they have children [only allowing 2 people to visit at a time] because the children will want to come in with their parents. But we work round that and let them in. They kind of swap round with each other, they are usually ok, they have never voiced an opinion that it's upset them..." (nurse 2)

“...No information is given over the telephone...it is one of my bug bears as a sister that the volunteered family member who is next of kin is only told the accurate information and then it is passed down so they 
don't ring up all at the same time...you get a family, and they ring up, ring up, ring up. We explain that it is important that the nurse is not taken away from the bedside that they are there to care..." (nurse 5)

“...It is a bit cold but it is necessary [buzzer entry system]...violence and aggression has grown considerably in health. So we have to have that system. So if you have got relatives who are howling and screaming and you've other patients that's not good for them either. So there has to be a system where they can't actually barge in the unit, which they could do before..." (nurse 5)

Staff are aware of the difficulties that are present, but many of them are necessary, even though they are not ideal, for example, a buzzer system protects staff and other patients from intruders or difficult visitors. It is the easiest way to deal with the situation, despite the fact that it seems impersonal.

\section{Improvements}

Suggestions or thoughts for improving the system include a larger number of smaller waiting rooms. Staff, not relatives, made these suggestions, and they relate to relatives' well being. The relatives were more concerned with the difference in level of care when the patient was transferred than with their own needs. This reinforces the conclusions that relatives and staff have different perceptions of their needs (ONNeill Norris \& Grove 1986).

“...I think if there was more money, possibly allow patients to stay over more. I sometimes think here, having that one big waiting room is not particularly nice. When you are sort of grieving and you are upset and 
you have got to share it with the world and his wife. And if you have got families who have a large amount of relatives, I feel very sorry for the, if you are then the one person who is sitting in the waiting room, you do feel, and quite often people loiter outside because they don't feel comfortable to go into the waiting room..." (nurse I)

Nurses think relatives should be updated regularly, "...maybe the doctors could speak to them a bit more and be a bit more down to their level. But most of them are pretty good actually at just speaking to them in bog standard language and usually one of us will stay behind and ask them did they understand everything? I know relatives would like to stay by that bed space all the time but sometimes it is just not appropriate for them to be there and it is not good for them to see things..." (nurse 2)

“...I think you need to have, I think you need to have, regarding the medical side of things, I think you need to have people who are willing to talk to relatives on a regular basis and up date them..." (nurse 3)

A suggestion about a screen to sometimes shield staff from relatives was also made. This was with the aim of easing transition from critical care to the ward, to try and make relatives less dependent on the staff,

“...we should have a screen, we are always there, you can't be anywhere else and patients see that you are always there and do get very demanding..." (nurse 1)

"...We talk about follow up clinics for patients, they should have a patient and relatives clinic together. And I think out of what they would 
want and what they see and the information we give them, was it jargonistic, too much, too little, what why should we deliver should come from the patient and the relative really. We are only measuring what we think..." (nurse 5)

These suggestions were made from the point of view of one unit. In terms of national units some already have similar provisions.

\section{Patient Care}

The only aspect of patient care that staff discussed was relatives' involvement in care. Involvement in Patient Care

Staff agree that relatives could be an asset to patient care, but also acknowledge that they are still not given the opportunities, "...some relatives really like to get involved, and it helps, they can come in and they can wash, help wash. I think we probably don't use, use is probably the wrong word, but utilise their abilities, perhaps they would like to be a bit more involved..."(nurse 1)

One nurse and a relative, who worked as a nurse, both agreed that it would be beneficial for relatives to be allowed to be involved in ward rounds and have access to more information.

“...then the question begs should they be part of the round. Because we should be should be working in participation with the patient and the family. I have no consequence with that. To me that would be quite glorious really..."(nurse 5) 
Although this idea would be beneficial for relatives because they would be more involved and more aware of their patient's condition, it would have implications for patient confidentiality for other patients on the unit.

\subsection{Limitations}

This was an exploratory study and the sample size was small. As expected, recruitment of relatives was difficult due to practical ethical restrictions because of the sensitive nature of the topic. One consequence of this was the relatives were predominantly female and therefore may not be totally representative of all the relatives who visit critical care units. All the staff were from the same unit, therefore the results should be treated with caution. Additional-information, for example, level of staff experience, was not recorded. This may have been a useful variable. These factors will be taken into account when further research is carried out.

\subsection{Conclusions}

Previous research investigated relatives' needs and staff's ability to assess those needs, primarily within the quantitative paradigm using survey research (e.g. (Heyland et al., 2002; Henneman et al., 1992; Forrester et al., 1990). This study illuminated the overall experience for both staff and patients using a qualitative approach.

Staff generally perceived, quite accurately, relatives experiences of the hospitalisation of a family member in a critical care unit and there was a high degree of congruence between staff and relatives' views. Contrary to what has been suggested in the literature relatives' psychological distress did not emerge as a salient issue. 
A sense that the media prepared these relatives for this situation, was common to all, but led to unrealistic expectations in some cases. The theme of torn priorities was also a widespread. Different emphasis was placed on avenues of support by relatives. Relatives of brain injured patients placed more importance on external organisations, possibly because there are not organisations available for every type of illness, whereas brain injury is a specific condition.

Relatives were keen to be involved in patient care. Staff acknowledged that it would be beneficial to have relatives involved in patient care, but barriers persist. If there is agreement that relatives involvement in patient care could be valuable it must be questioned why it does not occur.

Relatives of patients with brain injury faced problems due to brain injury being a secondary condition. It appears that care was centred on the primary reason for admission, often meaning that brain injury was initially overlooked. Due to the length of time that had elapsed between the relatives critical care experience and their interview problems with brain injury may have become more apparent, thus they have since developed the perception that that patients received inappropriate care often because they felt the hospital did not have the facilities to provide the appropriate care for the brain injury.

There was general dissatisfaction among both staff and relatives with the process of disseminating information. Relatives seemed to gain the information they required in a very inconsistent fashion, as illustrated when the relative had to ask if her husband was in a coma. Information should be given in small episodic events and then it should be checked that relatives' have understood (Zainal \& Scholes, 1997). The amount of information that relatives require is very individual and nurses are aware 
that some people want more information than others. The ability to assess this individual need is an ongoing nursing challenge. Millar (1994) emphasized the importance of 'working with' not 'working on' a family in congruence with the move towards holistic nursing. Staff in this study were aware of the importance of providing information, yet some acknowledged that they did not volunteer it. Reported reasons for this were resource and staffing issues. Reasons for this barrier to information giving may need further exploration.

Nurses are central to critical environments; they are an integral part of the support and communication networks and may be in the best position to implement changes and highlight any problems. Improving consistency of communication would reduce anxiety and facilitate healthier interactions between staff and relatives. As with most research this study raises significant areas for further investigation including: an analysis of information and communication systems available to relatives in the critical care environment (see Hughes et al, 2004). A communication audit could also be used to improve practices at a local level.

Exploring and recognising families' preconceptions about hospitals and their perception of illness may facilitate healthy interactions between staff and relatives. Communication and information giving is taught during training for most medical professionals. This study highlights the need for continued reflection and emphasis on this area throughout careers.

\subsection{Summary and Further Developments}

Relatives did not indicate that they found their critical care experiences for the most part psychologically traumatic. This is a positive reflection on the critical care units 
and the staff. One of the main recurring themes relates to the consistency of communication: giving and receiving information. This study illustrates that although staff are aware of how and what information should be given to relatives (i.e. frequency of information, by whom it is delivered, the detail necessary), the relatives themselves did not always report receiving it in that fashion. Throughout this study staff discussed what should be happening. It would be interesting to know whether this best practice is outlined in a policy document for the unit, or if staff know about what they should be doing from another source, for example, learning through experience. As highlighted in the literature, many studies fail to take account of nursing practices and unit characteristics, for example, overall unit philosophies on families (Hickey 1990); these are factors that could strongly influence relatives' experiences.

The literature is clear that relatives have difficulty seeking and absorbing information in a crisis (Bowman 2000) and as such, written information for relatives is recommended by staff in this study. Guidelines have been laid out by Department of Health (DoH) (Department of Health 2000b) in support of this. Phase II aims to address some of these issues by investigating the extent to which critical care units nationally provide information leaflets for relatives and have policies concerning the care of relatives. It will also examine the content of these leaflets and polices and the extent to which they meet DoH recommendations. 
Chapter Five

PHASE II: AN ANALYSIS OF INFORMATION AVAILABLE TO RELATIVES AND POLICIES CONCERNING RELATIVES IN CRITICAL CARE 


\subsection{Introduction}

As highlighted by the literature review, there are many gaps in research concerning relatives within a critical care environment. Much has changed since many of the studies were carried out and there are numerous trans-cultural differences between the UK and the US, where most of the research in this area has occurred.

It is well known that information reduces anxiety (Coulter 1989; Paul 2001). It has been found that the provision of pre-operative information (McGaughey \& Harrisson 1994a) and pre-operative teaching reduces anxiety of patients and relatives due to have planned surgery (Lynn-McHale et al. 1997). However, many admissions to critical care units are unplanned and admitted as an emergency. This creates a group of people who are not psychologically prepared for critical illness or the critical care environment. It is crucially important that suitable information is also provided to the families of this group.

Although satisfied with the care their patient received, relatives interviewed in Phase I were dissatisfied with the information they received and communication processes by which they received it. Relatives felt they received information in an inconsistent fashion and nurses perceived that the amount of information relatives required was dependent on individual differences, misjudging these differences may be a cause of that inconsistency. A possible solution could be to provide relatives with written information thus minimizing differences in the process of information giving. There has been an increase in written information for patients but evidence suggests that the quality can often be poor. The information rarely reflects the latest research evidence or acknowledges patients uncertainties or outlines their options (Carlisle 2003). The extent to which any of this information is directed at relatives is unknown. Relatives 
and patients needs may be very different. It is important that they are addressed adequately.

The most recent government policy relating to adult critical care services is the Comprehensive Critical Care document published in 2000. This review states that 'while no patient would wish to need critical care, those who experience the service, and their relatives [italics mine], should be confident that they have received the best possible care' (Department of Health 2000a). This demonstrates an acknowledgement that a patient is part of a family unit and critical illness has an extended impact.

The document sets out guidelines about information for patients, relatives and friends' (Department of Health 2000a). It acknowledges the distressing nature of the environment and the need for psychological and emotional support for both patients and relatives. It outlines that senior staff together with chaplains and professional and lay counsellors should provide this support.

It also recommends that information provided to relatives within critical care units should describe the service provided and explain the purpose and operation of common pieces of equipment. It should also provide information about the facilities available, descriptions of staff involved in care, important telephone numbers, relevant local and national organisations and chaplaincy services.

Bowman (2000) advocates that in a crisis situation, such as being in a critical care environment, relatives may have severe difficulty seeking, processing and retaining information because, as a person's level of arousal increases, the ability to process complex information decreases (Rosenzweig, Leiman, \& Breedlove 1996). Some staff in Phase I acknowledge that they do not always offer information voluntarily and furthermore, the same information would need to be repeated in lay terms on a regular 
basis. Mirr (1991) found that despite the fact that family members could repeat the information given to them by medical staff, when asked what the information meant it was obvious that they did not comprehend it. This illustrates the importance of having comprehensible written literature that people can take away and refer to at a later date. McGaughey and Harrisson carried out two studies (1994a; 1994b) with the aim of producing a booklet to meet the needs of patients and relatives in critical care, in Northern Ireland. They concentrated on preoperative information and undertook a small survey of 20 hospitals in the UK. They had 14 responses. The following study is of a much larger scale and concentrates on unplanned admissions.

The Comprehensive Critical Care review (Department of Health 2000a) suggests that the recommendations made should be completed within 3 to 5 years. The following study looks at whether any of these recommendations have been implemented.

\subsection{Objectives of Phase II}

The aim of this study was to gauge an overall view of the provisions available for relatives, at the current time, across general critical care units in England by conducting an analysis of

hospital policies concerning relatives and

information available to relatives.

A secondary aim is to see whether and to what extent these recommendations outlined in the Comprehensive Critical Care review have been implemented nationally, 3 years since the review was published. 


\subsection{Methods}

A letter was sent to 210 general critical care units across England. These were all of the general critical care units in England as listed on the Directory of Emergency/ Critical Care Units. The letter explained the purpose of the study and requested a copy of any policy documents the unit may have concerning relatives and a copy of any leaflets the unit may have for relatives. An attachment was sent with the letter for the unit to return if they did not have the required information (see Appendix G for letter and attachment). A self addressed, freepost envelope was also included to encourage a response.

The results were collated and analysed for basic descriptive statistics using the software package SPSS version 11.5.

When entered into the spreadsheet, the data was separated into subcategories. For each response the geographical location and the presence of a policy and/or a leaflet was recorded. The policy information was then split into topic areas e.g. admission procedures, discharge procedure. The same occurred for the information in the leaflet. Categories included visiting hours, explanations of equipment and information about support available. The main researcher determined the categories using the information received and the guidelines laid out in the Comprehensive Critical Care Review (Department of Health 2000a). Another researcher validated the categories.

The 'readability' of a randomly selected sample of $20 \%$ of the leaflets was measured using the Gunning's Fog Index (1952). This procedure was performed manually. The Fog index was calculated using the following formula:

Average words per sentence + percentage of long words 
Long words were words were defined as words with either 3 or more syllables when spoken. Long words that were excluded were words beginning with a capital letter, words made up of short words run together e.g. however and words that had -ed as a third syllable.

\subsection{Results}

\subsubsection{Description of sample}

The sample consisted of 210 general critical care units across England. Two units had merged since compilation of the database, therefore the total sample was reduced to 209. Of these, 119 hospitals responded. Although one hospital indicated that it had a leaflet it failed to include a copy and was therefore discounted. The sample analysed consisted of information from 118 hospitals; a 56\% response rate.

Figure 10 illustrates the geographical spread of the responses. The South East includes London. The differences are largely to do with the general location of hospitals. 


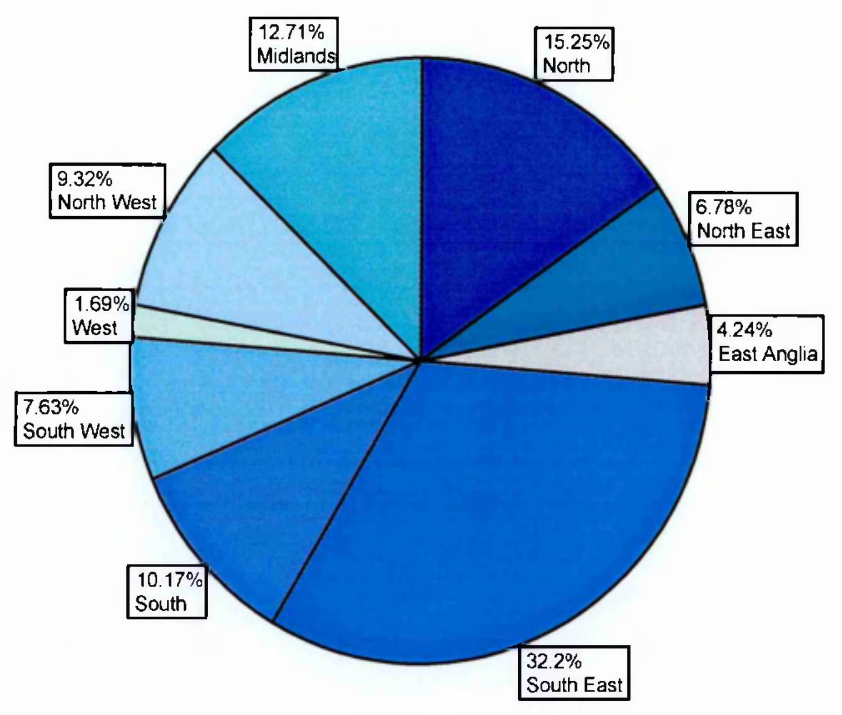

Figure 10-Geographical location of responses (\%)

\subsubsection{Data Analysis}

Eighty Four percent (99) of the units had a leaflet of some description for relatives. However these varied greatly in standard and amount of information provided. Only $9 \%(10)$ of the units had some form of official policy concerning relatives. Of those who responded $15 \%(18)$ of the units had neither a policy nor leaflet for relatives and $7 \%(8)$ of the units had both a policy and leaflet for relatives.

\subsubsection{Information leaflets}

Of the $84 \%$ of units that had a leaflet, $9 \%(9)$ of them used the leaflet printed from www.brake.org.uk. Seventy one per cent of these units had 1 leaflet, $18 \%$ had $2,10 \%$ had 3 and 1 unit had 4 different leaflets for relatives. Table 4 shows the type of information contained in leaflets. 
Table 4 - Summary of the type of Information contained in the leaflets for relatives.

\begin{tabular}{|l|l|}
\hline Category & $\%$ (number of units) of leaflets that contained \\
& the information \\
\hline Combined ITU and HDU leaflet & $35(35)$ \\
Admission & $33(33)$ \\
What is critical care and what to expect & $36(36)$ \\
Equipment & 42 (42) a further 10\% had this information \\
& displayed on a notice board instead of in the \\
Staff & $63(62)$ \\
Operations & $10(10)$ \\
Drugs & $15(15)$ \\
Facilities for relatives & $81(80)$ \\
Overnight room & $65(64)$ \\
If a patient dies & $14(14)$ \\
Transfer procedures & $53(52)$ \\
Recovery process & $31(31)$ \\
Visiting hours & $92(91)$ \\
Direct telephone line & $81(80)$ \\
Chaplain & $65(64)$ \\
Other sources of support for relatives & $41(41)$ \\
Emotional information for relatives & $36(36)$ \\
\hline
\end{tabular}

These figures can only give general ideas of the type of information relatives are provided with. For example, only a minority of units address the issues of death $(14 \%)$ and/or recovery $(31 \%)$. However, they do not indicate how well the issues are dealt with. A clear example is the information on equipment. Some leaflets go into great depth about all the different machines on the unit and their uses, others merely comment that

"...During your relatives stay in the Critical Care Department they may be attached to a variety of machines/monitors..." (visitors" information booklet for the critical care department) 
Each type would have been classed under the category of equipment. In addition to those leaflets that had information about equipment, a further $10 \%$ of the units had information on a notice board. Although this is better than nothing, it does little to help with the known poor retention rate of information that people have in a crisis. Similarly, under the staff category, some leaflets named the staff on the ward and their role, others merely commented that you might find nurses, doctors and physiotherapists. Using names helps to personalise the experience.

Emotional information for relatives refers to information about relatives touching the patient, whether the patient can hear them and information about how to look after themselves, such as getting adequate rest and eating properly.

\subsubsection{Readability of information leaflets}

The Fog test scores on the randomly selected $20 \%$ sample ranged from 21.69 to 34.18 , with a mean score of 27.55 . It is recommended that for information of this type the readability score should not exceed 20 . The leaflet from www.brake.org.uk scored 26.01. The 8 leaflets analysed by McGaughey and Harrison (1994b) had Fog scores ranging from 29 to 35 (mean $=31$ ), so, although still not ideal, it appears to have improved since 1994. However, their results have all the problems associated with small sample sizes and they acknowledge that their results are not generalisable. 


\subsubsection{Content of policy documents}

Policy documents refer to official explicit policies that units had regarding the management of relatives. Nine per cent of units had an explicit policy. Most policies were about the care of relatives. These include effective communication skills, passing on frequent and accurate information to the relatives, taking time to talk to them and making them feel able to express fears and anxieties. Table 5 shows the type of information each policy includes.

Table 5-Summary of the type of information included in policy documents.

\begin{tabular}{|c|c|c|c|c|c|c|c|c|c|}
\hline Policy & $\begin{array}{l}\text { D } \\
\text { 을 } \\
\frac{\bar{n}}{3} \\
\frac{0}{0}\end{array}$ & 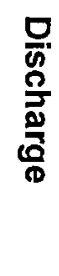 & 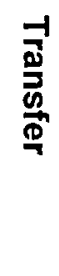 & 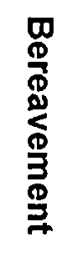 & 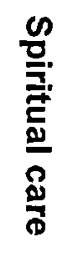 & 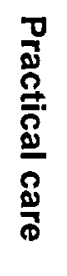 & 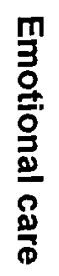 & 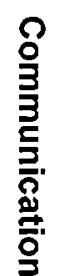 & $\frac{\widehat{m}}{\vec{F}}$ \\
\hline
\end{tabular}

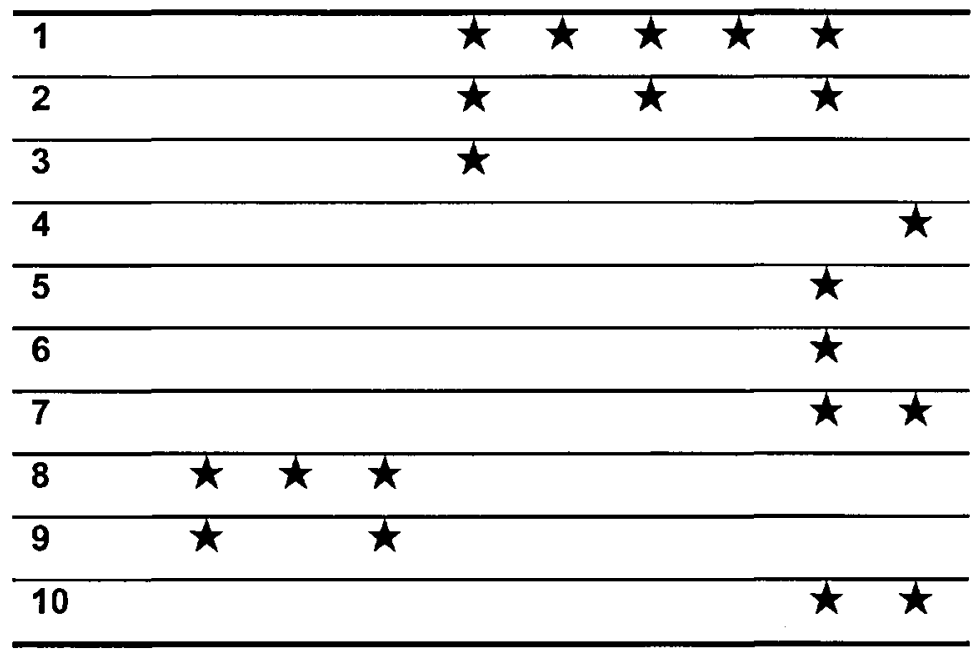

Information about admission, discharge and transfers is self-explanatory. Bereavement includes information prior to and after death. Spiritual care refers to information about chaplaincies and spiritual avenues. Practical care covers aspects such as facilities and routines. Emotional support includes elements such as providing 
a calm and safe environment for relatives, listening, supporting, allowing relatives privacy and being aware of the anticipatory grieving process and basic coping mechanisms, checking that relatives understand what they are told and reassurance. This is an important area that receives little attention in as far as policies are concerned. Communication looks at nurses' ability to communicate effectively and document that communication.

As with the leaflets there was much variation in the content of the policies. Ten policies were analysed. As can be seen from table 2, policy 7 was concerned solely with bereavement. Although an important aspect that deserves attention, there are other equally important areas of critical care that deserve the same attention. It was particularly concerned with specific actions as opposed to ensuring relatives well being; for example;

“...locate bereavement folder...In there you will find a slot for 'week commencing...' Put your card in an addressed envelope..."

There is no doubt that specific instruction for nursing staff is beneficial but a balanced medium needs to be achieved.

Policy 2, is at the opposite end of the spectrum and only mentions bereavement by saying;

“...if there is no hope of recovery ensure that significant others feel that patient will have a peaceful and dignified death..."

Policy 1 encompasses both elements:

“...the nurse will try to help the patient/relative prepare for death by involving spiritual and religious contacts or those able to help psychologically using all resources such as counselling skills...none 
of these will be employed if it is against the wishes of the patient or relative..."

It then lists the appropriate people to contact for the nurses' reference. The policy continues in this vein for all aspects of care prior to and after death. The same policy covers other aspects of care of relatives i.e. practical care about facilities available, spiritual care, and emotional support. This policy is the only one that has a strong emphasis on emotional support.

Six of the policies referred to initial contact with the relatives after admission. Three of these pinpointed a time span in which contact should be made. It ranged from 5 minutes to 6 hours. Policy 1 outlined that a care co-ordinator for relatives' spiritual care be appointed within 24 hours. The other two made no reference to any time span.

Other policies address different issues such as admission, transfer and discharge procedures. Policies 8 and 9 contain very technical and procedural information on admission, discharge and transfer. Relatives are referred to almost as an afterthought. The closest policy 9 gets to referring to a relative is as a "substitute decision maker."

Policies 2 and 5 place more emphasis on communication in the sense of

"...telephone is answered using the agreed ICU greeting..." or

"...all members of the team are pleasant polite and approachable..."

Policy 2 acknowledges poor retention rates,

"...information should be repeated to significant others at regular intervals...due to significant others reduced ability to retain large amounts of information..." 
Policy 7 predominantly talks about visiting with a passing reference to communication. The information in the policy echoes what is to be found in the information leaflet for relatives.

\subsubsection{Additional information available}

Some units had a variety of additional information available to relatives, such as a leaflet on MRSA (Methicillin Resistant Staphylococcus aureus) infection or relative feedback questionnaires. Table 6 is a summary of the additional information available to relatives.

Table 6-Summary of additional information available for relatives.

\section{Additional information}

Leaflet on brain stem death

Video of what to expect in a critical care unit

Information folder in waiting room

Leaflet on bereavement

Information about critical care on notice boards in waiting room (NB this does

not include information about equipment)

Relative feedback survey

Leaflet about MRSA infection

PALS Leaflet

Sympathy card sent by the unit to the relatives after a patient has died

Additional information available on hospital website

Leaflet on tracheostomys

Pre-operative leaflet

Patient diary

Leaflet on post mortems

Leaflet on resuscitation
Number of units

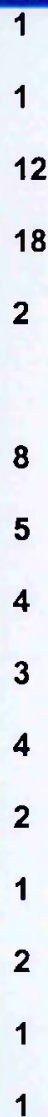

1 
It is not known how the above information is disseminated, whether it is left in waiting rooms, or handed out to relatives when appropriate. It is interesting that the most frequent additional leaflet is one on bereavement. Hopefully, this would be handed out as and when required. Of the 12 units that had an information folder in the waiting room, 4 did not have a leaflet. As mentioned previously, relatives have difficulty absorbing information during crisis situations; it is advisable that they have information to take away with them, so that they can refer to in a calmer moment.

\subsection{Discussion and Conclusion}

From the results, it can be seen that the $16 \%$ of the units that do not provide any form of leaflet are not complying with the Department of Health recommendations. Table 1 shows that units that do have leaflets are not always relaying enough information to comply with the government guidelines. Only $31 \%$ of units describe the service. Only $36 \%$ of units explain equipment in the leaflets; however a further $10 \%$ did have that information on a notice board in a relative's room. More units included information about facilities for relatives (69\%) and just over half gave information about who the staff were (53\%). The majority of units included information about the critical care units direct phone line (68\%). Fifty four per cent of units included information about chaplaincy services, but only $35 \%$ included information about other areas of nonreligious support, locally and nationally.

There are also large variations in the appearance and presentation of the leaflets. Although this may seem trivial the appearance of a leaflet affects how easy it is to read and how likely it is to be read. The importance of presentation has been acknowledged for patient information (Carlisle 2003). This would also apply to relative information. Guidelines are available for presenting text in the most 
accessible way for its readers (Royal National Institute for the Blind 1998). These include, using $12 \mathrm{pt}$ font and producing information on yellow, white or cream nonglossy paper to improve legibility. These guidelines also warn against giving unnecessary prominence to words by using capitals, bold and underlining.

The Audit Commission (1999) stated that the most basic requirement was a booklet available to relatives that contains general information. They claim that $91 \%$ of critical care units had such a leaflet. This study illustrates a more reserved figure of $84 \%$.

It is apparent that in many parts of the country guidelines set out by the government on information that relatives should receive are not yet being met. The www.brake.org.uk leaflet that some hospitals use appears to cover all the specified areas. However, its Fog Index is not as low as it perhaps could be. As it is a general leaflet it would also be useful to have a supplementary leaflet, including information specific to that hospital, e.g. visiting times and staff names, which some of the hospitals did include. The Comprehensive Critical Care Review (Department of Health, 2000a) indicated that there were many good examples of material produced by professional bodies. The Brake leaflet is one such example. The Fog Index scores illustrate that, despite information being available, it may not be as accessible to its audience as would be desired. The website www.criticalcareinfo.org is a useful addition to information for relatives. It is a free downloadable booklet developed partly from the BrakeCare booklet, but has the opportunity to include personalised information about local units. It is supported by the Intensive Care Society and the RCN Critical Care Forum.

The Centre for Health Information Quality (2000) states that good quality information is clearly communicated, evidence based and involves patients in the development 
process. This would also be applicable to relatives. It is important that they are involved in the development of information aimed at them.

Readability tests such as the FOG index are a good indication of the ease of which a piece of text can be read. However, they can only act as a guideline. The scores are only an indication of readability level and do not take into account relatives previous knowledge of the medical terms regarding their patients illness and some short words may not be understood by most readers (Kenny, Wilson, \& Purves 1998). Despite this readability tests can be useful tools for preparing information. For this type of information a FOG index score should not exceed 20.

The inconsistent nature in which relatives receive information is reflected in the national inconsistency concerning policies and leaflets relating to them. From this analysis it can be seen that nationally there is huge variation, over the amount and quality of information relatives have access to and receive. Also, there is no consistent nationwide policy of how staff should deal with relatives when they have a patient in critical care. This implies that the attention a relative receives when in an critical care environment varies greatly depending on which hospital their patient is admitted to. Obviously, information that is, or is not, available to relatives has no reflection on the care that a patient receives whilst in critical care.

An additional problem associated with written information is that it may not always correlate with verbal information provided (Paul 2001). Information leaflets may be most useful if staff on the unit go through the leaflet with relatives rather than just handing it out. This may serve not only to assist relatives understanding but also to refresh staff on the content of the leaflet with the aim of improving or maintaining consistent information. Another external pressure influencing individual units is that 
Trusts may place constraints on content and format of information for relatives due to Clinical Governance.

There is explicit information available about developing and producing patient information (e.g. Duman \& Farrell 2000). This could be used by critical care units and applied to relatives. It outlines points to take into consideration when developing information policies and leaflets.

According to the DoH guidelines laid out in the Comprehensive Critical Care Review (Department of Health 2000) leaflets should contain at a minimum information to describe the service provided and explain the equipment. It should also include information about facilities available and staff involved in the care of the patient. In addition, information should be available about relevant local and national organisations and chaplaincy services. Relatives in Phase I were unspecific about exactly what information they would have liked to have received. This is what will be investigated further in Phase III.

These results could be used to bring leaflets into line with existing policies. They could then be subjected to readability tests to ensure that it is comprehensible for its audience. There is also a need for explicit policies on addressing the needs of relatives that can be used as a basis from which staff can work. This will help teams to develop a consistent approach and will offer guidance to newly qualified staff. 
Chapter Six

PHASE III: RELATIVES INFORMATION NEEDS IN CRITICAL CARE 


\subsection{Introduction}

This phase of the study seeks to clarify some of the issues that arose from Phases I and II. The results from Phase I indicate that relatives received information in an inconsistent manner. Although generally satisfied with their experiences, this was an area of concern to them. In line with these findings, Phase II showed that there were still $16 \%$ of units did not provide any written information for relatives and only $9 \%$ had a policy for staff recommending guidelines on how to care for relatives. In order to discover where inconsistencies in information giving and communication processes lie and whether failure to meet Department of Health guidelines affects relatives experiences, this phase sought to assess whether the content of information leaflets was what relatives truly required and to explore in greater depth relatives reactions to the communication process, the information they received, and what information they would like to have been given.

It is established that information reduces anxiety (McGaughey \& Harrisson 1994a) under stressful circumstances. A detailed discussion of previous literature exploring the importance of communication and existing processes can be found in Chapter $2, p$ $33-36$.

As with Phase I, this study is divided into two sections. Part A investigates relatives' experiences and Part B investigates staff perceptions.

\subsection{Objectives of Phase III}

The aims and objectives of this study are:

1. To ascertain relatives' specific views on the type, amount and nature of information they receive; 
2. To explore staff views on the information gained from relatives' interviews;

3. To compare the differences between the needs of relatives' in planned and unplanned admissions;

4. To compare the differences between needs of relatives' and reasons for patients' admission;

5. To explore whether the Department of Health (DoH) guidelines laid out in Comprehensive Critical Care: A review of adult critical care services (Department of Health 2000a) reflect what staff and relatives feel is required.

\subsection{Methods}

Phase III adopted grounded theory methodology. Data were collected from relatives using research interviews. The data gained from the interviews were then presented to nursing staff using focus groups. This enabled preliminary feedback to be given to the staff and provided staff opinions on the issues raised. Data from the focus groups was fed back into the interview schedules with relatives. For a detailed discussion of grounded theory and the appropriate methods see Chapter 3, p57.

\subsection{Recruitment, data collection and data analysis}

\subsubsection{Relative recruitment}

An adult sample of relatives aged 18 or over, was recruited through two Critical Care units in a large teaching hospital in London. Eight relatives were recruited through a general critical care unit and ten relatives through a specialist neurological critical care unit. Analysis of the data showed that similar themes arose from relatives from both units and therefore all the relatives were treated as one sample. 
A relative was someone who was considered significant to the patient and was determined by observing who visited the patient most regularly. Relatives who had been informed that their patient was likely to die were not approached. The patient had to have been in the critical care unit for 48 hours or longer and was over 18 years.

Staff on the unit identified those patients without a negative prognosis and the researcher approached the relatives and handed them an information sheet. The relatives were then approached the following day to ascertain if they were willing to participate. There were 18 interviews in total; six relatives were male and 12 were female (see table 7 for participant data). Four of the participants requested that another family member sat in on the interview for moral support. 
Table 7-Participant data for relatives

\begin{tabular}{|c|c|c|c|c|c|c|c|c|c|c|}
\hline $\begin{array}{l}\dot{0} \\
0^{n}\end{array}$ & $\begin{array}{l}\mathbf{d} \\
\mathbf{d} \\
\mathbf{d} \\
\mathbf{0}\end{array}$ & 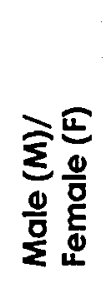 & 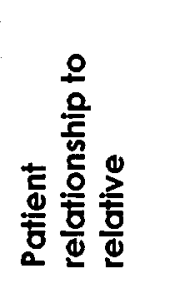 & 过 & 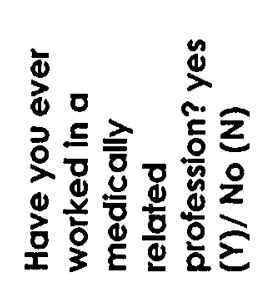 & 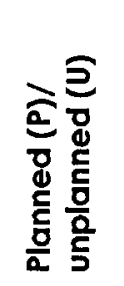 & 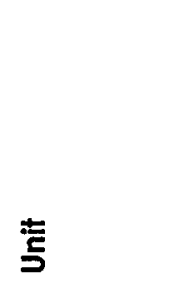 & 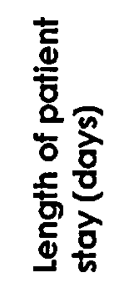 & 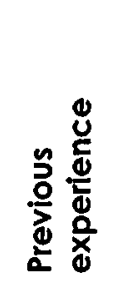 & $\begin{array}{l}\bar{z} \\
\dot{z} \\
\Sigma \\
\Sigma \\
\Sigma\end{array}$ \\
\hline 1 & $M$ & & Mother & 48 & $Y$ & $\mathrm{U}$ & General & 4 & $Y$ & \\
\hline 2 & $\mathrm{~F}$ & & Husband & 75 & $N$ & U & General & 17 & $N$ & \\
\hline 3 & $\mathrm{~F}$ & & Husband & 44 & $Y$ & U & General & 7 & Y & \\
\hline 4 & $\mathrm{~F}$ & & Husband & 53 & $N$ & U & General & 28 & $N$ & \\
\hline 5 & $\mathrm{~F}$ & & Husband & 50 & $N$ & $U$ & Neuro & 7 & $N$ & \\
\hline 6 & $\mathrm{~F}$ & & Father & 62 & $Y$ & $P$ & General & 4 & $Y$ & \\
\hline 7 & $\mathrm{~F}$ & & Husband & 62 & $N$ & U & Neuro & 14 & $N$ & \\
\hline 8 & $M$ & & Daughter & 46 & $N$ & $U$ & Neuro & 3 & $N$ & \\
\hline 9 & $M$ & & Wife & 63 & $N$ & $U$ & General & 5 & $N$ & \\
\hline 10 & $F$ & & Husband & 74 & $N$ & U & Neuro & 5 & $N$ & \\
\hline 11 & $\mathrm{~F}$ & & Father & 42 & $N$ & U & General & 5 & $N$ & \\
\hline 12 & $\mathrm{~F}$ & & Mother & 51 & $Y$ & U & Neuro & 10 & $N$ & \\
\hline 13 & $M$ & & Father & 43 & $N$ & $\mathrm{U}$ & Neuro & 6 & $Y$ & \\
\hline 14 & $M$ & & Daughter & 42 & $N$ & $\mathrm{P}$ & Neuro & 18 & $N$ & \\
\hline 15 & $\mathrm{~F}$ & & Husband & 59 & $N$ & $\mathrm{U}$ & Neuro & 7 & $Y$ & \\
\hline 16 & $\mathrm{~F}$ & & Son & 45 & $Y$ & $\mathrm{U}$ & Neuro & 14 & $Y$ & \\
\hline 17 & $\mathrm{~F}$ & & Husband & 75 & $N$ & $\mathrm{U}$ & General & 3 & $N$ & \\
\hline 18 & $M$ & & Son & 45 & $N$ & $U$ & Neuro & 14 & $N$ & \\
\hline
\end{tabular}


Of the total number of relatives approached, an additional eight were transferred from the units before there was an opportunity to follow them up, three relatives were given information sheets but their patients deteriorated and it was considered inappropriate to continue and three declined to participate. Of those who elected not to participate two stated that it was too upsetting a time for them and one said that their patient was about to be transferred and they did not have time to participate.

\subsubsection{Staff Recruitment}

An adult sample of qualified critical care nursing staff were recruited through the same two critical care units as the relatives. Eight staff were recruited though the general critical care unit and 12 through the neurological critical care unit. Six nurses from general critical care units in Surrey were recruited for a pilot focus group. This data was also included in the study. Again, when analysing the data, similar themes arose from staff from both units and therefore all staff were treated as one sample. (see tables 8 and 9 for participant data). Staff experience of working in critical care ranged from 6 months to 20 years, with the average being 5.8 years experience, this data was unavailable for three participants. The staff grade ranged from $D$ grade to $G$ grade including one practice facilitator and one sister. The most common grade was D. The time and location of the focus groups for that day was advised at a series of staff handover meetings and participation was voluntary. Two groups were scheduled per day and if staff were willing to participate they met at the prearranged time and place. 
Table 8 Participant data for staff from the neurological unit

$\begin{array}{cccc}\text { Ps No. } & \text { Age } & \text { Years experience } & \text { Staff Grade } \\ \mathbf{1} & 26 & 2 & \mathrm{D} \\ \mathbf{2} & 44 & 11 & \mathrm{G} \\ \mathbf{3} & 46 & 19 & \mathrm{G} \\ \mathbf{4} & 41 & 15 & \text { Practice Educator } \\ \mathbf{5} & 30 & 3 & \mathrm{D} \\ \mathbf{6} & 32 & 4 & \mathrm{D} \\ \mathbf{7} & - & - & - \\ \mathbf{8} & 35 & 10 & \mathrm{~F} \\ \mathbf{9} & 25 & 1 & \mathrm{D} \\ \mathbf{1 0} & - & - & - \\ \mathbf{1 1} & 49 & 20 & \mathrm{~F} \\ \mathbf{1 2} & 37 & 2 & \mathrm{D}\end{array}$

Table 9-Participant data for staff from the General Unit

$\begin{array}{cccc}\text { Ps No } & \text { Age } & \text { Years Experience } & \text { Staff Grade } \\ 13 & 30 & 3 & \text { E } \\ 14 & 22 & 0.6 & \mathrm{D} \\ 15 & 41 & 15 & \mathrm{G}-\text { Team Leader } \\ \mathbf{1 6} & 30 & 3 & \mathrm{E} \\ \mathbf{1 7} & 36 & 8 & \mathrm{E} \\ \mathbf{1 8} & 30 & 2 & \mathrm{E} \\ \mathbf{1 9} & 31 & - & \mathrm{E} \\ \mathbf{2 0} & 28 & 0.6 & \mathrm{D} \\ \text { P21 } & 27 & 1.5 & \mathrm{D} \\ \text { P22 } & 37 & 5 & \text { sister } \\ \text { P23 } & 43 & 4 & \mathrm{E} \\ \text { P24 } & 26 & 1 & \mathrm{E} \\ \text { P25 } & 25 & 0.5 & \mathrm{D} \\ \text { P26 } & 26 & 1.6 & \mathrm{E} \\ \mathbf{P}=\text { Pilot study, not held in the same unit } & \end{array}$




\subsubsection{Data Collection}

The data from relatives was collected through semi-structured interviews. Consent was sought and participants were informed of their right to withdraw at any stage. Responses were recorded on a standardised proforma. Relatives were shown the interview notes immediately after the interviews and asked to verify that what had been recorded was an accurate representation of what had been said and if so were then asked to sign the proforma. All interviews lasted approximately 30 minutes and took place on the critical care units either in a quiet room or a seminar room.

The data from staff was collected through tape-recorded focus groups. Consent was sought and participants were informed of their right to withdraw at any stage. At the completion of the focus group participants were asked to sign the consent form a second time, to confirm their agreement that what had been recorded could be used (dual consent).

One pilot focus group was carried out with nurses who worked in a number of general critical care units across Surrey. This focus group was used to test the viability of this particular method which had not previously been used for any phase of this study. It was held at the University of Surrey and six nurses attended. The group was successful as a method but it was decided that with six nurses present the focus group lasted too long (1 hour). By reducing the number of nurses by half, it was thought that it would only last for 30 minutes; a more acceptable length of time for nurses to be away from the unit. The data was included in the main study.

All other focus groups took place on the critical care units and lasted approximately 30 minutes. Four focus groups were held with neurological unit staff and three with 
general unit staff. One tape from a focus group with staff on the neurological unit was inaudible and unable to be used.

\subsubsection{Data Analysis}

All data were analysed using comparative analysis techniques and NUD*IST, a qualitative software package. For a full discussion see chapter 3 p 60 .

\subsection{Part A: Results and Discussion from Relatives' Interviews}

\subsubsection{Description of sample}

The sample consisted of 18 participants. These were relatives who had a family member in either the general or the neurological critical care units at a large London teaching hospital.

\subsubsection{Themes}

Initially the data was analysed inductively in order to establish patterns, themes and categories. To do this, the data was coded line by line through open coding. The codes were refined through axial and selective coding. From this themes and categories were formed by comparing codes for similarities and differences according to their properties and dimensions and grouping them accordingly (Strauss \& Corbin 1998). During the process of coding the data, and as more data became available through continued interviews, the titles and descriptions of concepts were refined. The later generation of hypotheses about the relationships between the concepts involved deductive processes. For a detailed discussion of this see Chapter $3, \mathrm{p} 60$. The themes that were developed fit into six main categories (see figure 11);

- Information giving, 
- Relatives reactions to the patient

- Relatives perceptions of staff

- Relatives perceptions of the unit

- Relatives' reaction to their situation

- Practicalities and procedures

These categories are comprised of various related concepts e.g. the 'information giving' category is made up of concepts such as 'type of information' and 'information givers'. Each category and its relating concepts are discussed in detail below.

'Information giving' and 'practicalities and procedures' expand upon the Phase I categories 'information' and 'unit practicalities' respectively. 'Information giving' explicitly relates to the gaps in the results from Phase II concerning what information, how and by whom, relatives wished to receive. Concepts from the Phase I 'relatives' category such as 'emotional reactions' and 'coping and support' are also built upon in this phase in the 'reactions to their situation' category in the concepts 'emotions', 'coping' and 'perceived social support'.

These concepts interact to illustrate the process that relatives experience in critical care. This process is illustrated in Figure 12. 


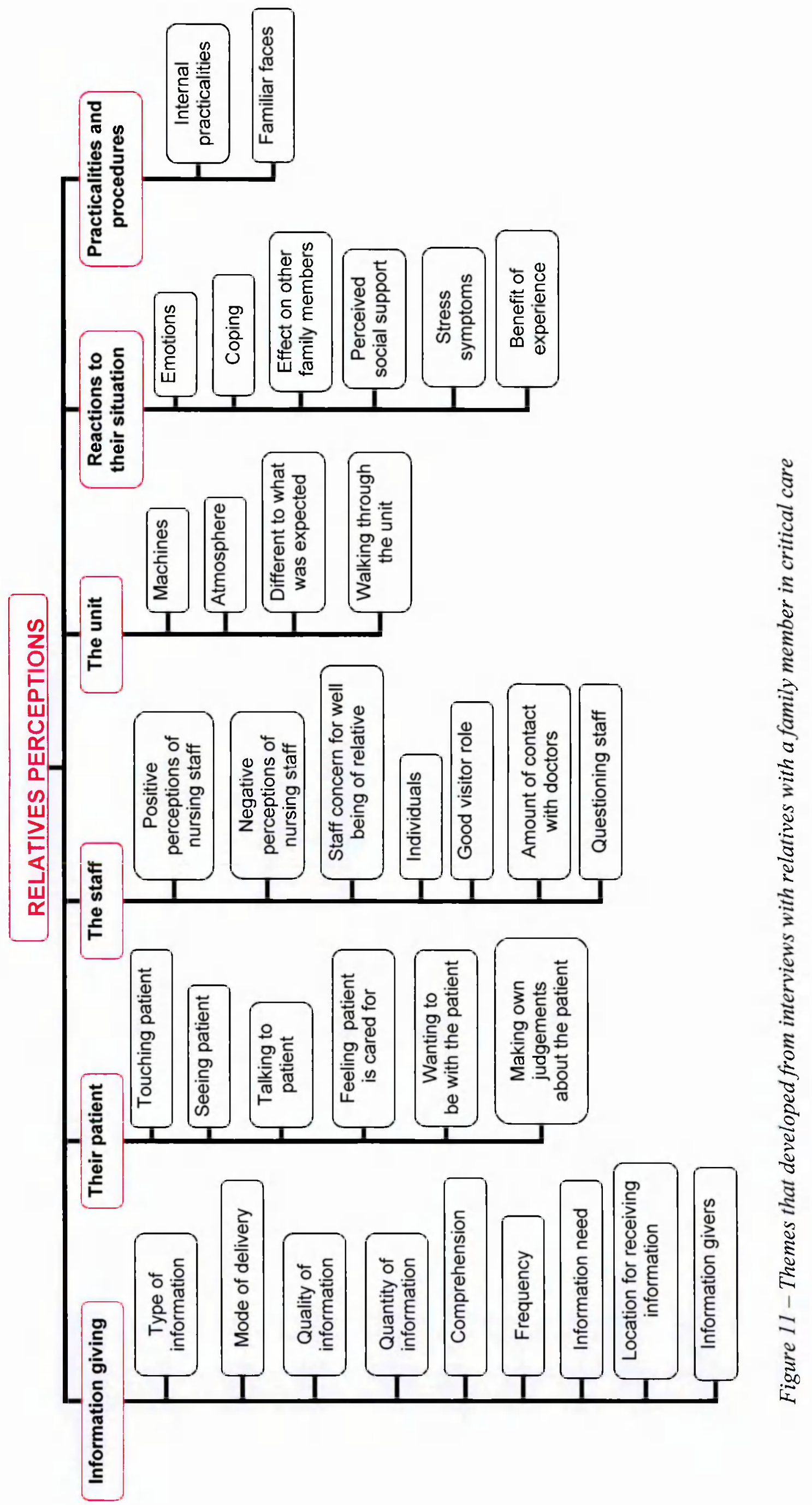




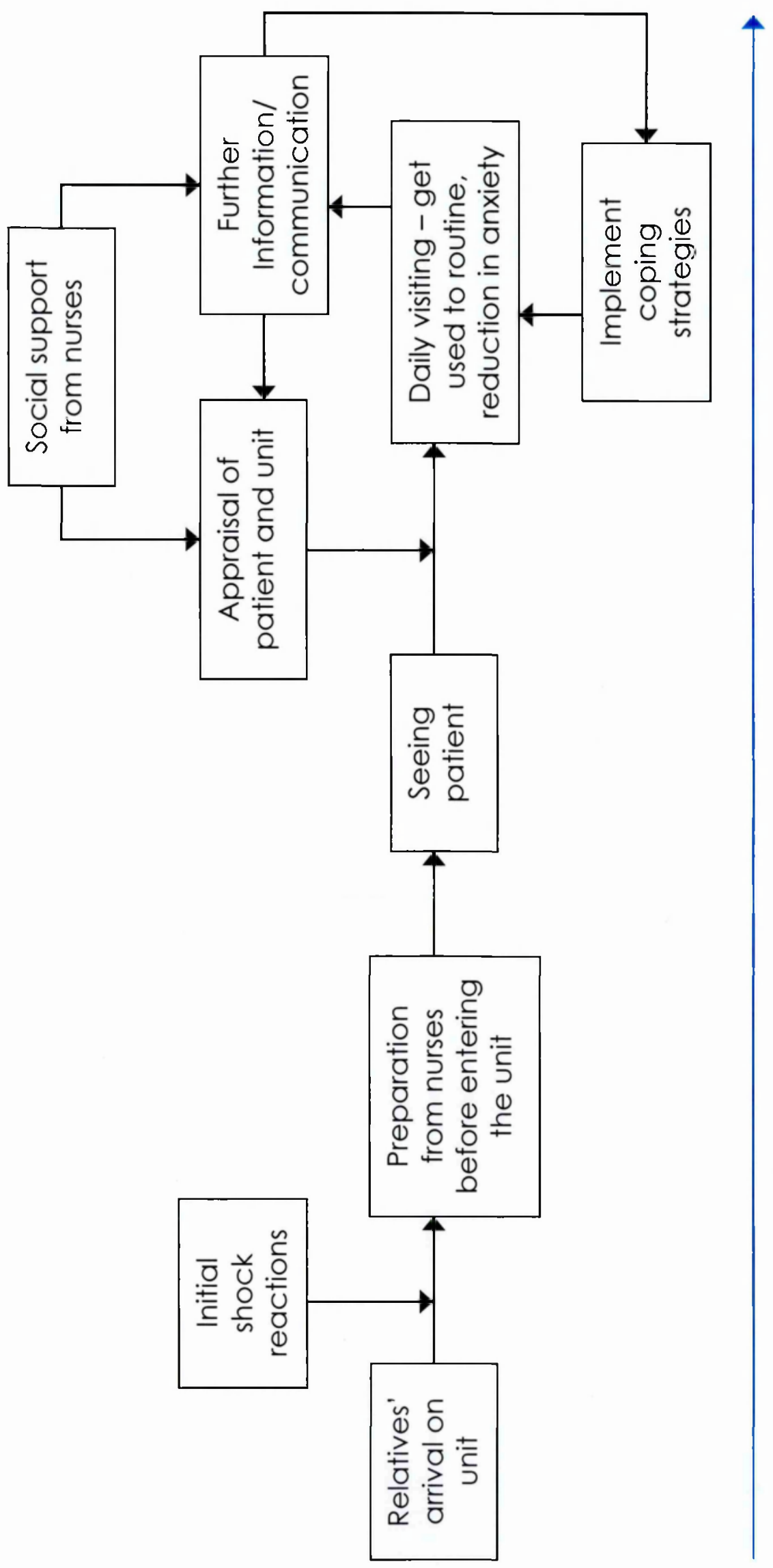

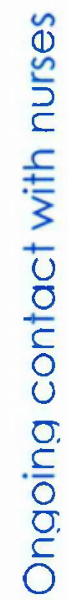

ำ 


\section{Information Giving}

One of the main aims of this phase of the study was to identify a relative's specific information requirements. This category is defined by nine main themes: type of information, mode of delivery, quality of information, quantity of information, comprehension, frequency, information need, location for receiving information and information givers. These categories will be discussed in turn below.

\section{Type of information}

Relatives received a mixture of factual information and reassurance. When there was no change to the condition of their patient, they did not receive any new information at all. The following illustrative quotations are in response to the question 'what type of information have you received?'

$$
\begin{aligned}
& \text { "...what type of treatment he was getting, condition, prognosis..." } \\
& \text { (relative 2, female, general unit) } \\
& \text { "...factual and reassurance. We found that helpful. The nurses were } \\
& \text { open with us. They can't give miracles but they have given hope. They } \\
& \text { have been honest and taken away any doubts..." (relative 16, female, } \\
& \text { neurological unit) }
\end{aligned}
$$

Although it can not be concluded from either Phase I or II how much verbal reassurance staff gave relatives it is useful to reiterate that only $36 \%$ (36) of information leaflets and $10 \%$ (1) of policies included emotional support for relatives. Relatives accepted that staff could not change the reality of the situation but they perceived that there were times when the only information they received was negative. Relatives reported that they needed something positive to focus on, which 
reinforces studies that have shown how important hope is to aid successful coping strategies (Patel 1996) (see Chapter 2 p21 for a more detailed discussion on hope) and supports findings from Phase I where relatives also acknowledged how important it was for them to be able to hope. The literature strongly supports this concept (Coulter 1989;Zainal \& Scholes 1997).

“...one doctor only ever gave negative information all the time. I didn't want to hear negative information all the time. It would have been nice if he was a little positive..." (relative 3, female, general unit)

\section{Modes of delivery}

Relatives received all their information verbally, either face to face or by telephone. Relatives were grateful that they were encouraged and able to ring the unit at any time to enquire about the patient.

“...they are very good at allowing ringing..." (relative 12, female, neurological unit)

One unit had restricted visiting hours, from $2-8 \mathrm{pm}$, it was felt that not to hear anything at all from $8 \mathrm{pm}$, when visiting ends, until $2 \mathrm{pm}$ the following day, when visiting would start again was a long time, and although most people telephoned in the morning, they believed it would be reassuring if they could be telephoned when the patient had settled down for the night.

“...a later call just to say he is settled would be nice..." (relative 17 , female, general unit)

Despite one of the units having an information leaflet for relatives, only one of the relatives interviewed had received any written information (about her husband's 
condition) and she was in the unit without an information leaflet. Contrary to the Department of Health guidelines (Department of Health 2000a) that recommends relatives be given written information about the practicalities of the unit, eight of the 16 relatives who did not receive any information did not want any. They spent so much time on the unit, that they were satisfied with receiving information verbally. Of the 8 that did want some written information, only four wanted information about the specifics of the unit. One would have liked directions to the hospital (she did not live locally), three wanted information about the specific condition of the patient, whilst two would have liked written information about operations that had been performed.

\section{Quality of information}

Most relatives said that the information they had received had always been consistent and they considered it helpful. Relatives valued this consistency highly,

“...we have spoken to a doctor. There have been no problems. We have been given all the details of his condition. I can't fault them..." (relative 6, female, general unit)

“...very [consistent]. They never held anything back. We are always reminded that things change..." (relative 8, male, neurological unit)

However there were exceptions,

“...everyone says different things..." (relative 4, female, general unit)

On occasions some relatives perceived that the medical staff they were talking too had not read the patient notes sufficiently and were not fully informed about their specific case, however, this was not a common complaint, 
“...sometimes you feel that the person you have spoken to has not read all the notes and they don't know everything..." (relative 12, female, neurological unit)

There was also some confusion when there were multiple problems with the patient, “...fairly consistent. Different doctors have different ideas for treatment, because they have different priorities..." (relative 18, male, neurological unit)

\section{Quantity of information}

There were varied views regarding the quantity and adequacy of information. Some relatives thought that they only received information if they were in the right place at the right time,

“...we pay attention because we are after any snippet of information they can give us..." (relative 17, female, general unit)

Others were very satisfied with the amount of information they received,

“...excellent. They pro-actively gave information. I didn't have to ask any questions..." (relative 1, male, general unit)

"...but the information was incredible. Not everyone is as lucky as C.

She has improved day by day..." (Relative 8, male, neurological unit)

There were specific areas, e.g. the equipment around the bedside, which some relatives would have liked explained to them. The following relative who was not satisfied with the amount of information she received sought information from other avenues, 
“...nobody has actually explained them [machines and equipment] to me, you pick up different bits from different nurses. I would have liked to have had them explained..." (relative 15, female, neurological unit)

“...just that it is going to be a long time. It's not terribly reassuring. I phoned a helpline and they weren't terribly helpful either...I was at the hospital all the time, so I was having to rely on other people to get it [information] for me..." (relative 15, female, neurological unit)

In some instances it was believed that the delivery of information depended on the character and experience of the member of staff the relatives were dealing with,

“...they didn't contradict themselves, but it depends on how confident the nurse is as to how much information they pass on..." (relative 15, female, neurological unit).

\section{Information need}

Although relatives were satisfied with information they received about the patient, they considered it was very difficult to find out about practicalities that impacted on their daily lives, for example car parking permits, accommodation and refreshment facilities. Often people were aware that a system must exist because of their experiences at previous hospitals. They also believed that they should not bother the nursing staff with these types of questions as it was not part of their role and could be construed as wasting the nurse's time.

"...no one told us about the car parking permit, we were told by [previous hospital]" (relative 17, female, general unit) 
"...the practicalities have not been volunteered...I have had to ask.

Once I asked it came across quickly but had a not already know there was a system perhaps I wouldn't have asked. [eg parking accommodation]..." (relative 15, female, neurological unit)

It also appeared that as time passed relatives wanted to know more information than they had during the initial phases, but the level of information that were actually receiving was remaining constant.

"...nurses told us as much as they know and that was enough to cope with for the time being. As time goes on I do want to know a bit more..." (relative 5, female, neurological unit)

\section{Frequency}

Relatives received daily information from the nurse at the bedside because they spent a lot of time at the units, however information from doctors was less frequent. There is ample literature that suggests relatives in a crisis need to be given continual, simple information for them to absorb what they have been told (Zainal \& Scholes 1997)

"...he [the consultant] said that they were doing their best but he will keep us informed every couple of days and he did do..." (relative 4, female, general unit)

\section{Comprehension}

All relatives said that they understood what they were being told, 
"...[the information was] very plain and very clear..." (relative 2, female, general unit)

Anything they were unsure about they soon picked up, or else it was explained more clearly to them. This is contrary to other studies that have found that in $50 \%$ of cases, relatives do not understand what doctors have told them about prognosis, diagnosis and treatment of the patient (Pochard et al. 2001).

\section{Location for receiving information}

Information was either given by the bedside, slightly away from the bedside or in a quiet room. Relatives associated negative implications with the quiet room, as they perceived that if they were invited in there, staff would impart bad news. There was also a feeling that if the patient was even slightly aware of their surroundings, relatives did not want information given within earshot of the patient, in case it was not good news. They wanted the information first, so that they could decide whether or not the patient should be told. In Scotland before the Adult Incapacity (Scotland) Act 2000 relatives did not have the right to make decisions regarding their patient. Officially that was the domain of doctors, although many relatives were not aware this was the case (Booth et al. 2004). In England, relatives have no legal right to make decisions about their patients, although it is common law practice. The Draft Mental Capacity Bill (2003) (Authority of the House of Lords 2003) will do more to make this common law statute. Previous legislation has only dealt with people who suffer from mental disorders as oppose to those people who are temporarily incapacitated for any other reason. 


\section{Information givers}

Most of the information relatives received came from nurses by the bedside. Contact with doctors remains inconsistent. Often relatives only see doctors if they happened to be visiting when the doctor came round. Relatives were often confused by the number of different people they saw and commented that some of the doctors did not introduce themselves when they approached the bedside. Relatives viewed information from health professionals as legitimate and this supported previous studies on different groups of patients (e.g. cardiac patients - Wiles 1998).

“...the doctors and nurses tell it like it is, sometimes they tell you what you don't want to hear..." (relative 17, female, general unit)

“...I have had poor contact with doctors. When I have asked to see a doctor, it has been very haphazard, you either have to make an appointment which is never definite and usually involved hanging around for about 2 hours, or if you just happen to be there. It is very haphazard. I realise that they have other people to see but how long does it really take to explain a few things there and then and not say make an appointment..." (relative 15, female, neurological unit)

\section{Reactions to the patient}

Relatives had mixed reactions to their patient, which was influenced by whether they had any previous experience of critical care or whether they had ever worked in a medically related profession. This category is divided into six concepts; touching their patient, seeing their patient, talking to their patient, feeling their patient is cared for, wanting to be with their patient and making their own judgements about the patient. 


\section{Touching their patient}

Most relatives were happy to touch their patient. There was an acknowledgement that if they were not comfortable touching their patient, it was not because any restrictions had been placed upon them, but because they were afraid that touching would interfere with the lines or increase the chance of infection,

“...I am happy to touch but there is a concern of infection, especially where the tubes go in and things. It is better to keep hands off. I don't feel that she is alienated. We are well aware of what everything is for. We have a biological, medical background..." (relative 12, female, neurological unit)

If the relative was a spouse or parent, there was a strong feeling that they wished they could comfort their patient physically,

“...I just wish I could have cuddled him. We are not restricted and not stopped from touching him. We couldn't have asked for better treatment..." (relative 17, female, general unit)

“...I felt comfortable touching my wife. I felt an amazing outpouring of love..." (relative 9, male, general unit)

\section{Seeing their patient}

There were mixed reactions to seeing the patient. Some relatives were shocked and scared by the sight of their patient being so gravely ill and attached to multiple wires. Others were just pleased that they could be present and that their patient was receiving the care that they obviously needed, 
“...when I arrived I didn't really see the unit. I was more concerned with my daughter. Seeing her with all the wires and stuff was really hard to accept. I knew it must be doing her good. But with all the surroundings, I felt like I was in fairyland..." (relative 14, male, neurological unit)

"...I was more in shock [than his wife] seeing him [their son]. I still don't like it..." (relative 18, male, neurological unit)

\section{Talking to their patient}

Relatives sometimes felt awkward talking to their patient because it was a one-way conversation. One relative when approached to take part in the study agreed commenting,

"I have only been here five minutes and I have run out of things to say already, if my wife were here we would have chatted [to each other]..." (relative 8 , male, neurological unit)

This was a frequent remark. Relatives believed that they should talk to their patient, but did not always know what to say,

"...I talked to her. I believe that you should talk to people just in case they can hear you..." (relative 1, male, general unit)

"...I am fine about touching him, but it is hard to talk to him, but it is getting easier..." (relative 16, female, neurological unit) 
Feeling their patient is cared for

Relatives wanted to feel that their patient was genuinely cared for. They placed emphasis on personalised care and attention to personal detail, such as nicknames.

"...officially he is called J, but everyone calls him Jack. It took a long time to get the nurses to take note. He doesn't respond to J..." (relative 17, female, general unit)

"...I felt like my husband was being treated like a piece of meat not a person..." (relative 3, female, general unit)

In Molter's (1979) original study the need to feel that the hospital personnel cared about the patient was ranked as the second most important need. This has been replicated in subsequent studies, although not always placed so highly (e.g. Mathis 1984; Quinn, Redmond, \& Begley 1996).

\section{Wanting to be with their patient}

It emerged that relatives just wanted to be physically close to the patient. This allowed them to feel as though they were doing something useful, whether or not this was a reality. They wanted to be physically close in case of any sudden change, either positive or negative,

“...it was awful not seeing her while they were getting her ready, but you know that they have got her best interest at heart...I just want to be with my daughter..." (relative 8, male, neurological unit)

"... just want to be with him..." (relative 4, female, general unit) 
“...we were very anxious and would have liked to have been here even though there was nothing we could have done..." (relative 5, female, neurological unit)

Relatives demonstrated that they wanted to be with their patient by visiting every day. They wanted to be involved in patient care, some by just wanting to know what was happening, others by being more practically involved.

\section{Making own judgements about their patient}

Relatives did not always believe the information they were given and felt that watching the patient was a more important indicator of whether they appeared better or worse. For certain illnesses, this can, at times, be deceptive.

"...I make my own judgments because I can see her. If they say she is better and she doesn't look better then I don't really accept it..." (relative 14, male, neurological unit)

\section{Perceptions of staff}

Relatives had varying perceptions of the staff. This category has been divided into six main concepts; positive perceptions, negative perceptions, staff concern for well being of relatives, individuals, good visitor role, amount of contact and questioning staff. These are discussed below.

\section{Positive Perceptions of nursing staff}

Relatives were very impressed with the staff cohesion on both units and felt that the units were run well and staff worked well as a team, 
“...they are very knowledgeable about the patients. It is a well drilled unit..." (relative 13, male, neurological unit)

"...I see them doing a good job...the staff seem to work well together and they are very co-operative..." (relative 1, male, general unit)

"...the staff are very enthusiastic...they are a good team..." (relative 12, female, neurological unit)

All the relatives were very impressed with the quality of care their patients received.

“...everything has been done to the best of everyone's abilities. It restores you faith in the NHS. You can see where the money is being spent. They are on top of their jobs..." (relative 13, male, neurological unit) "...they have taken great care of him. I am satisfied with the treatment. I don't know what other treatment he could have got...I have lots of admiration for the doctors and nurses..." (relative 2, female, general unit)

“...the level of competence was remarkable..." (relative 9, male, general unit) “...the nursing staff are excellent. Reassurance with experience..." (relative 7, female, neurological unit)

Relatives were also touched by the kindness of the staff, "...he is 84 years old and has had so many tests. They have shown a lot of kindness, nothing is too much trouble..." (relative 17, female, general unit)

"...I feel as though we have been treated special..." (relative 8, male, neurological unit) 
Relative 8 illustrated his point by describing the following event,

“...the phone wasn't working in the waiting room, so they said come in here [to the unit] and use this one. Nothing seems to be too much..." (relative 8, male, neurological unit)

The professionalism staff displayed was seen as a reflection of the quality of care that patients received. Relatives were particularly impressed with this,

“...you can't get better than the professionals..." (relative 8, male, neurological unit)

“...the staff are first class...they are very professional. No one shied away from telling us the truth. But they did it delicately. Everyone who came to the bedside introduced themselves..." (relative 13, male, neurological unit)

For staff to introduce themselves at the bedside was an issue that was important to relatives, who wanted to feel involved in care of their patient. If staff introduced themselves it enabled them to feel like an important and useful part of the process.

\section{Negative perceptions of nursing staff}

Not all perceptions of the staff were positive; some relatives had negative opinions, although these were in the minority. For certain relatives, breach of trust was an important issue for certain relatives. If staff failed to do something that they had said they would do, relatives felt let down and subsequently found it hard to trust that member of staff. The following relative was advised that she would be telephoned at home if there was any change in her husband's condition. When she arrived at visiting time, she discovered that her husband had been ventilated that morning. She deemed this a major change and was upset that she had not been informed in advance. 
Throughout the interview she appeared angry about this and continually reiterated that the staff could not be trusted to do what they said they would,

“...going home was the last thing I wanted to do. I hate them for that ...they said that they would ring as soon as anything happened. They lied...I rang in the morning and came in about $11 \mathrm{am}$. When I arrived he was ventilated. No one told me in advance. I was shocked because I thought that I would be rung if there was any change..." (relative 3, female, general unit)

\section{Staff concern for well being of relatives}

None of the relatives in this study were asked by the nursing or medical staff if they were coping with the situation. Many commented that it would have been reassuring to know that they were cared about, as well as the patient, particularly those relatives who were on their own,

"...no one has ever asked if I am coping...I would have found it helpful, it would have been reassuring that they were concerned..." (relative 15, female, neurological unit)

“...we haven't been asked how I have been coping. I think I would have found it useful..." (relative 17, female, general unit)

In a study on the role of critical care nurses in relation to families it was found that $38 \%$ of critical care nurses did not believe that it was realistic to expect them to care for the emotional needs of relatives of critically ill patients (Hickey \& Lewandowski 1988). However this study is quite dated. The concern of staff for relatives was not always perceived as helpful by the relative. One relative commented adversely on 
how the nursing staff made her go home and get some rest and she resented them for it because she just wanted to be with her husband,

"...then they insisted on me having some sleep in the quiet room because I hadn't slept..." (relative 3, female, general unit)

By suggesting that relatives rest, staff show concern for their physiological well being. This reiterates work by O'Neill Norris and Grove (1986) who found that families' perception of their needs differed from nurses' perceptions of family needs. In particular, relatives placed less emphasis on physiological needs than nurses.

\section{Individuals}

Relatives were aware that nurses and doctors on the units had their own characteristics and that every individual was different.

"...it is down to individuals, $\mathrm{X}$ was very professional and caring. She looked beyond the monitors. Once I had to point out to someone that his mask was full of saliva..." (relative 17, female, general unit)

\section{Good visitor role}

Relatives seemed to fall into a 'good visitor role'. They were grateful for what the nurses were doing for them and felt that they did not want to pester the nurses too much or irritate them. The next quotation refers to a relative who felt that here husband was becoming agitated because the nursing staff were going to change his ventilator. 
“...I felt that I had to pester a bit today...I was allowed to come in outside visiting hours to calm my husband down..." (relative 3, female, general unit)

“...I don't mind talking, but once you start talking you don't stop and you don't want to get on their [the nurses] nerves..." (relative 10, female, neurological unit)

This is similar to 'sick-role behaviour' (Sarafino 1998) whereby patients adopt certain behaviour when they believe they are ill in order to fill a 'sick role' and thus get well. Relatives in this situation feel that they must fulfil a role which includes visiting their patient and giving support to their patient, without interfering with the nurses' role of caring for their patient. If relatives do something that they perceive to be outside this role, for example remind the nurses to do something, they then perceived that they have overstepped pre-drawn boundaries.

\section{Amount of contact with doctors}

Relatives had contact with nurses every day but contact with doctors was variable,

"...We have always seen the doctors. We have had good contact with doctors...the doctors and nurses tell it like it is, sometimes they tell you what you don't want to hear...contact with the doctors is us grabbing them..." (relative 17, female general unit).

“...the doctors gave us the serious information. We are happy with the contact we have had with doctors..." (relative 18, male, neurological unit)

Some relatives were satisfied with the amount of contact they had with doctors however, there was an underlying assumption that if relatives wanted to see a doctor it 
was necessary to ask. Some relatives accepted this and implied it was their responsibility if they had not asked to see a doctor,

“...I have only seen one doctor, but I haven't asked to see another doctor..." (relative 10, female, neurological unit)

“...I don't really know who are doctors and who aren't...I spoke to the surgeon. He explained that the operation had been successful. I don't know who they [the doctors] are. If I wanted to speak to the doctors, I would have made a point to find them..." (relative 14, male, neurological unit)

“...we hadn't spoken to the doctor until yesterday when we spoke to an anaesthetist. We would have liked to have had a little more contact with doctors. We could have pressed more often. But I think it was more that I didn't ask. I don't like asking when I am here on my own in the week..." (relative 5, female, neurological unit)

These findings are similar to those in Phase I where relatives only had limited contact with doctors. The acceptance that it was the relatives fault for not asking is another element of the 'good visitor role' discussed above. Others relatives only saw doctors if they were by the bedside when the doctors came around,

“...We haven't had to ask to see a doctor, we have caught them by the bedside. The anaesthetists don't introduce themselves..." (relative 12, female, neurological unit)

Doctors' introducing themselves to the relatives by the bedside is a theme that links a variety of concepts. When doctors did introduce themselves, it was seen as a positive reflection on the staff and contributed to the view that the patients were receiving a 
good quality of care, because it instilled confidence. When doctors did not introduce themselves, they appeared more elusive to the relatives. The fact that some relatives are unsure which staff are doctors reflects a lack of communication on the part of the doctors. This small measure of information may help to make relatives feel involved and in turn reduce some of the anxiety and confusion they feel at this time. Some relatives were dissatisfied with their limited contact with doctors,

“...I have had poor contact with doctors. When I have asked to see a doctor, it has been very haphazard, you either have to make an appt which is never definite and usually involved hanging around for about 2 hours, or if you just happen to be there. It is very haphazard. I realise that they have other people to see but how long does it really take to explain a few things there and then and not say make an appointment..." (relative 15, female, neurological unit)

Although relatives spent a lot of time at the units, they resented the perception that their time was unimportant. Another negative perception held by some relatives was that doctors were too busy to talk to them,

“...there is never a doctor to actually speak to you or to approach. They always seem to be too busy. When I asked to see a doctor the nurse said that there was no need and that there was nothing a doctor could do that she couldn't do..." (relative 4, female, general unit)

Relatives seek to obtain different types of information and reassurance from doctors than from nurses. 


\section{Questioning staff}

Relatives had differing attitudes towards asking questions. Most were more than happy to ask questions,

“...we were generally given information... I was happy asking questions but the nurses were forthcoming..." (relative 1, male, general unit)

"...I asked questions and got answers. Sometimes you did have to ask. Sometimes they volunteered information...I didn't feel that I shouldn't be asking [questions]. When he was very poorly I rang when I got home and very early in the morning and they were always happy to talk..." (relative 2, female, general unit)

"....in the morning when we first come in we tend to ask lots of questions about how he has been over night..." (relative 16, female, neurological unit)

“...every question I could think of asking has been answered confidently and accurately..." (relative 13, male, neurological unit)

However, there were two relatives who felt nervous and uncomfortable asking questions and, as such, felt that they were not receiving as much information as they perhaps could have,

“...yes, but I have to push myself. Like this morning the machine was beeping and it said that his blood pressure was 50 over 50 and it was a flat line and no one seemed to be doing anything about it, so I thought that I had to comment. I know that things can change so drastically. I 
appreciate that they deal with death all the time, but it is different when it is your family..." (relative 15, female, neurological unit)

“...I don't like asking when I am here on my own in the week..." (relative 5, female, neurological unit)

The relative below did not herself feel uncomfortable asking questions, but expressed doubts about what information they would have found out if they had felt awkward asking,

"...the nurses don't always volunteer information. Sometimes you

feel like you are asking too many questions. We mainly ask, but some

do volunteer. It is hard to say what would have happened if we hadn't

asked questions..." (relative 12, female, neurological unit)

Many of the questions asked by relatives concerned their patient's condition, how their patient had been during the night, what they could expect and the nature of the treatment their patient was being offered.

\section{Perceptions of the unit}

The category includes relatives' perceptions of the machines on the unit, the atmosphere in the unit, their expectations and how it differed and relatives reactions to walking through the unit to reach their patient.

\section{Machines}

The equipment in critical care elicited two types of response; those who found it frightening and those who found it comforting. 
"...the unit was very impressive and I found the machines comforting, not scary..." (relative 9, male, general unit)

"...I ran out because I couldn't stand the sight of the machines..." (relative 4, female, general unit)

This theme was also raised in Phase I (see Chapter 4, p88). The effect of environmental factors on relatives is also discussed in detail in Chapter 2, p35. It is important that relatives have the functions of the machines and the environment explained to them to reduce their fear. Many relatives commented how nurses explained this to them,

"...the alarms are a concern. I have had the equipment explained to me..." (relative 7, female, neurological unit)

\section{Atmosphere}

One relative was interviewed on the neurological unit following the transferral of her husband from the general unit, also involved in this study. She commented that she found the general unit more frenetic than the specialist unit. However, those who had only been on the general unit, and had nothing to compare it to, did not comment on the hectic nature of the unit.

“...the atmosphere they created on the unit was very positive. Very good. Not dark and moribund but like they are enjoying their work. The doctors were very good..." (relative 9 , male, general unit)

Many relatives on the neurological unit commented upon the calm, relaxed atmosphere the unit had,

“...it was a very calm environment..." (relative 12, female, neurological unit) 


\section{Different to what expected}

A number of relatives commented that the unit was not what they had expected. Their expectations had developed through media coverage, previous hospital experiences or stories of others experiences,

“...I am very grateful, all you hear about is all the awful reports in the newspapers, but it isn't like that..." (relative 17, female, general unit)

"...just to be able to sit here with her and feel comfortable. I can't believe the relaxed attitude. They have cracked the nut here. I was expecting something more Victorian. It is great to know it is here...when my wife had the kids, it was all, you can't come in here like that. Do this. Do that..." (relative 8, male, neurological unit)

\section{Walking through the unit}

Some relatives found walking through critical care units very daunting. The position of their patient's bed on the unit influenced their reactions to their patient. When their patient was in a very critical condition, relatives found walking through the unit past other, equally critically ill patients, made them feel worse, but as their patient improved, they found walking past very critically ill patients made them feel better: the realisation that their patient was no longer as ill as other patients gave them encouragement. Relatives discouraged younger children from visiting as they perceived that walking past all the other critically ill patients would be too distressing for them,

"...it was horrific having to walk through the ITU. My son [12 years] won't come up because of it. But now he is getting better it is a relief 
to come through, because he is getting better and isn't as bad as that..." (relative 11, female, general unit)

“...I found the equipment and the unit a bit traumatic. It was walking through the unit..." (relative 6, female general unit)

\section{Reactions to the situation}

Relatives of a critically ill patient reacted to the situation differently. This category explores relatives' emotional reactions and coping mechanisms. It also includes the perceived effect the experience is having on other family members and perceived level of social support received by relatives. Finally it reviews stress symptoms exhibited by relatives and the perceived benefit of a previous experience.

\section{Emotions}

Relatives experienced a wide range of emotions throughout their exposure to critical care. These are fully outlined in Table 10. 
Table 10 - The range of relatives' emotions while a family member is in critical care

\begin{tabular}{|l|c|}
\hline Range of emotions relatives experience & Incidence from group of 18 \\
\hline Shock & 8 \\
Numbness & 2 \\
Fear & 5 \\
Distress & 5 \\
Feelings of unreality & 3 \\
Feelings of haziness or 'like a blur' & 2 \\
Anxiety & 6 \\
Uncertainty & 3 \\
Bewilderment & 3 \\
Anticipatory grief & 2 \\
Seeking blame & 1 \\
Hope & 6 \\
Relief & 2 \\
Gratitude & 3 \\
\hline
\end{tabular}

The most common emotions were those of shock, anxiety and hope. Initially relatives experienced shock but as they became familiar with the environment, this subsided in varying degrees.

\footnotetext{
“...I was shocked at first. I had never been into an intensive care unit before and I was amazed at the sight of the equipment..." (relative 2, female, general unit)
}

Even those relatives whose patients were planned admissions were still experienced shocked on entering the unit, 
“...I came in the first evening and it was a big shock. I have never experienced anything like that before..." (relative 14, male, neurological unit)

Feelings of anxiety were continuous once their patient was out of danger relatives became less anxious about whether their patient would survive and more anxious about what would happen next and how they would cope with it. This finding is supported extensively in the literature (Patel 1996). Hope protects people from despair (Coulter 1989) and gives relatives something positive to focus on in otherwise desperate circumstances. These emotional reactions were also reflected in the results of Phase I.

Coping

Relatives, in this sample, used one or any of three main methods of coping. Some relatives, whose patient was an elderly parent or part of an elderly couple, adopted a philosophical approach to the critical care stay.

"...you have to be a bit philosophical about it. He is 88 and he was only born with one foot, one kidney and one eye so he has done pretty well really..." (relative 6, female, general unit)

Previous life experiences contributed to this philosophical approach. If the patient or relative had been through worse before, this crisis was more readily accepted, “...he stopped breathing at night sometimes. It may sound hard, but it's not hard, but he has had prostate cancer and things, so you have to be a bit philosophical..." (relative 10, female, neurological unit) 
This is an emotion focused method of coping that uses cognitive process to minimise cognitive distress. For a detailed discussion of coping mechanisms (see Chapter $2, \mathrm{p}$ 24). Others relatives simply adapted to the situation by developing a routine. This is similar to some relatives in Phase I who were happier in the unit they knew, rather than having to adapt to a new routine somewhere they unfamiliar (see chapter $4, \mathrm{p} 87$ ),

"...I am getting used to it as the days go on, which is worrying because he was only supposed to be in for 24 hours..." (relative 11 , female, general unit)

"...it gets easier. There is always trepidation. My mood changes depending on my wife's condition..." (relative 9, female, general unit)

"...you get used to things, the unit and the routine. Everyone is very organised and you get to know the staff..." (relative 18, male, neurological unit)

Fitting into the routine of the unit is a problem focused coping mechanism aimed at the self, by developing new patterns of behaviour. Relatives felt that when events occurred which interrupted or changed their routine they went back to the beginning and had to start all over again,

"...I was worried at lunchtime, because I was told that the nurse would come out and get me, and I wanted to know why they hadn't just said 'wait 10 minutes'. It worried me, shook me. I am coping by just getting on with it..." (relative 10, female, neurological unit)

“...as time goes on, it becomes more routine, until there is another crisis, for example, when they told us what they were going to do, slow down her heart rate, that was nerve racking and the sudden 
calmness and the routine was gone and you were back to the beginning again..." (relative 12, female, neurological unit)

Others cope by thinking positive thoughts about the unit and the situation. This is an emotion focused mechanism of reappraising the situation.

“...I knew it must be doing her good..." (relative 14, male, neurological unit)

\section{Effect on other family members}

Some relatives expressed concern about the effect of the hospitalisation of the family member and the environment on other family members, particularly on children,

“...my children found it more difficult. They were very distressed.

They hadn't seen anything like it before. They didn't like walking past everyone else before they got to my father..." (relative 6, female, general unit)

This is linked into the category 'reactions to the unit' and more specifically the concept of 'walking though the unit.'

One relative explained that following a stroke, her husband had been in and out of hospital for six months and had been in critical care for four weeks. She was extremely concerned about the effect her husbands illness was having on her 13 year old daughter. Her daughter had missed a lot of school and did not like going to school as she did not like leaving her mother,

"...she has become very clingy. I think she is frightened in case she

loses both mum and dad. She is not normally out of my sight any more..." (relative 4, female, general unit) 


\section{Perceived social support}

As in Phase I the main avenues of social support for relatives were other family members, friends and the nursing staff. Contrary to Phase I the neurological unit relatives did not mention specific external organisations as a means of support, perhaps because none of the relatives had yet to experience the rehabilitation phases of recovery.

“...we [the family] have supported each other. You get upset. Family have been the main support..." (relative 13, male, neurological unit)

"...I was on my own. I was supported by the nurses on the ward...I got in touch with my son for some advice. He was very supportive, very strong...friends have been the main avenue of support..." (relative 9, male, general unit)

A few relatives perceived a lack of support, both emotional and otherwise, “...I was on my own and that wasn't good..." (relative 3, female, general unit) "...we need to convert the garage for him...his special wheelchair is too wide for the house. No one has come forward to help. We were told that we would get help..." (relative 4, female, general unit)

The relative above was advised that she was entitled to financial help, but she did not know how to go about accessing it and wanted someone to assist her with the process.

\section{Stress symptoms}

It was apparent that relatives were suffering from certain symptoms that are associated with high stress levels. It is known that anxiety and depression can have a 
major impact on a person's ability to make decisions (Pochard et al. 2001). It has been shown that the prevalence of anxiety and depression symptoms, in family members $(n=920)$ of critically ill patients, was $69.1 \%$ and $35.4 \%$ respectively. These symptoms were more predominant in spouses than other family members (Pochard et al 2001).

Relatives experienced a lack of concentration and problems with memory. When asked, many relatives could not recall members of staff they had come into contact with or information they had been told, particularly when they first arrived on the unit,

"...it goes in one ear and out the other..." (relative 14, male, neurological unit)

Relatives felt drained and tired having to visit the unit every day,

"...I am tired all the time. I know it is because I am stressed. Everything is an effort, everything hurts..."(relative 11, female, general unit)

"...I am finding it mentally and physically draining coming in every day..." (relative 14, male, neurological unit)

“...I have been here every day for about 8 hours a day. It's exhausting...." (relative 15, female, neurological unit)

Most relatives also experienced a range of sleeping difficulties and loss of appetite. The sleeping difficulties included trouble getting to sleep, trouble staying asleep and early waking,

"...I am not sleeping. I am calmer when I ring at 9am, because I know

I am coming in soon. It is a long time not to hear anything [from $8 \mathrm{pm}$ 
the previous night until $2 \mathrm{pm}$ when visiting hours start]" (relative 17 , female, general unit)

“...I have problems sleeping, I wake up very early...I have lost my appetite, but keep trying to eat. I feel sick. I worry..." (relative 10, female, neurological unit)

“...we had sleep problems the first few nights, but it is ok now...we lost our appetites to start with..." (relative 16, female, neurological unit)

Many of these are depressive symptoms but it is not possible to deduce from these results whether this is an acute reaction or a more clinical state. Previous studies have indicated that relatives can suffer from enduring depression following critical care experiences (Jones et al. 2004). Future research could investigate the extent to which these and other depressive symptoms decrease over time, if at all.

\section{Benefit of experience}

Relatives who had previous critical care experience or who had worked in a medical related profession found the critical care units less daunting. The relative quoted below was visiting his father who was in critical care, he explained how the unit itself did not intimidate him because he had visited been in critical care units previously when his brother had been in a car crash and when his daughter was born,

“...I am used to all the machines...machines are very familiar. I have no problems with that..." (relative 13, male, neurological unit)

Relatives with no previous experience of a critical care unit acknowledged that, if a similar situation were to occur in the future they would be better prepared for it, 
"...if I have to go through anything like this again, it will be better next time..." (relative 14, male, neurological unit)

\section{Practicalities and procedures}

This category shares similarities with the 'unit procedures' category that emerged in Phase I. It is divided into two main concepts; internal practicalities and familiar faces.

\section{Internal practicalities}

This concept covers four main topics: visiting, waiting facilities, refreshments and patient admission. The two units involved in this study have different visiting hours. The specialist unit had open visiting except between 12 and $2 \mathrm{pm}$, the general unit had set visiting hours between $2-8 \mathrm{pm}$. Relatives interviewed from the latter unit felt unhappy when they were asked to leave the bedside, for whatever reason, during visiting hours. There was a strong feeling that this eroded the perceived short amount of time they could spend with their patient. Similarly, if the relative arrived to visit their patient at the start of visiting time and was then not allowed into the unit immediately they felt that they were having their time taken away from them,

"...I think visiting time should be extended. They are very rigid when it finishes. Not being allowed in for 18 hours each day feels like 3 weeks. My husband feels the same. They are very strict about the times. I am not happy when I arrive at $2 \mathrm{pm}$ and then have to wait even longer, for example while they roll him. They made me wait 45 minutes. It doesn't take 45 minutes to roll someone. I am not in the way. I feel that I should be allowed in..." (relative 3, female, general unit) 
“...I don't like the visiting hours. It is horrible when you can't get in until $2 \mathrm{pm}$. I don't like it when you get here and they say that they are going to roll him and you have to wait even longer. One day they made me wait ages and I just walked in and waited outside the curtains. One day they made us wait outside for 2 lots of 20 minutes and once for 40 minutes. It was all for stuff that they could have done earlier. My son then had a row with the nurses about doing things in visiting times because they tried to make him leave at $8 \mathrm{pm}$. Some nurses let you stay a bit longer..." (relative 4, female, general unit)

Others did not like the restricted visiting hours because they lived far away. They felt that they could not reach the hospital quickly enough should the condition of their patient changed,

“...I don't like the visiting hours. I live an hour away and the children further and if anything deteriorated and we weren't here, we couldn't get here straight away..." (relative 17, female, general unit)

Patients also benefit from having relatives visit. One relative commented how one morning she decided to come in later than normal because she was exhausted and her husband became anxious that she was not with him,

“...yesterday I didn't come in until 11.30 and the nurse had rung me 3 times to ask when I was coming in, so my husband was obviously anxious that I wasn't there..." (relative 15, female, neurological unit)

Relatives from the unit with open visiting had more varied views. Some disliked being asked to leave temporarily, because it was felt that they nothing should be happening to the patient that they could not witness. Seeing procedures carried out 
also helped the relative to understand what was happening. Others were happy to leave if a procedure had to be carried out preferring not to see their patient in distress,

“...I object to being asked to leave when they want to carry out a procedure. I feel that they shouldn't be doing anything that they can't do in front of me and I need to know, I need to understand..." (relative 15, female, neurological unit)

"...when they have been doing a procedure and have asked us to leave, we have been happy to. We are very practical about it. We don't want to see her distressed..." (relative 12, female, neurological unit)

Relatives of conscious patients appreciated having a break. At the end of one interview a relative commented that he would slip out of the unit for a cup of tea, while his daughter would still think that he was in the interview.

“...the open hours, you need a break for a couple of hours. We come in all day..." (relative 13, male, neurological unit)

Both units in this study had relatives waiting rooms, outside but adjacent to the units. The general unit had an additional quiet room adjacent to the main waiting room. The neurological unit had a similar quiet room, in this case, inside the unit. There was mixed opinion as to whether these facilities were adequate. The main waiting rooms of both units were similar sizes and problems with the waiting facilities mainly occurred when the units were full and/or one particular patient had a very large family. This often intimidated family members of other patients,

“...the relatives' room is like a cattle yard. Today there are 13 people for one patient. There needs to be more control over the visitors room and some more privacy. If only two people are allowed in and one is 
waiting, it isn't very nice for the third person. It can be very intimidating. There is nowhere private to cry..." (relative 17, female, general unit)

"...the waiting room is not adequate for the amount of people who have to use it. It would be good to have a telly while you are waiting. It would just be something mindless to concentrate on. One Sunday there were 40 people in the waiting room for one patient. It looked like they had all just come from church and there were kids running around all over the place and half of them didn't even go in. My mother in law is 80 and when I bring her up she needs somewhere to sit..." (relative 4, female, general unit)

“...they need a bigger waiting area..." (relative 12, female, neurological unit) "...the relatives room is the best I have ever seen..." (relative 8 , male, neurological unit)

Relative 8 did not have any previous experience of critical care units.

Relatives were dissatisfied with refreshment facilities in the hospital. The main issue was the ability to obtain good food and drinks outside of normal working hours. Relatives spent a considerable period of time at the units, and found this problematic, particularly at weekends or late in the evening. Although both units had vending machines for hot drinks and confectionary close by, when these were out of order relatives felt that the alternatives were very limited. Although not a main concern of relatives, being able to get obtain refreshments was important to them, "...the restaurant is not open at weekends..." (relative 10, female, neurological unit) 
"...food outside serving hours is awful they should have a franchise, like the wild bean café or something..." (relative 12, female, neurological unit)

"...the machine [drinks vending machines] is out of order and the coffee shop was shut down over the weekend..." (relative 6, female, general unit)

The original interview schedule did not include questions concerning the availability of refreshments. This was a topic that arose repeatedly when, at the conclusion of the interview, relatives were asked if there was anything they felt had been overlooked, or that they wanted to add.

When a patient is admitted to a critical care unit there is an initial period of time in which relatives have to wait whilst medical staff and nurses stabilise and settle the patient before they are allowed into the unit to visit. Relatives are required to wait outside with little information about what is going on and nurses are faced with the dilemma of tending to their priority, the patient, or caring for relatives that they know to be anxiously waiting outside the unit.

Relatives appear to remember little about this initial period. When asked most could not remember the first piece of information they were given and usually guessed that it was a general overview. Nor could many remember the first member of staff they spoke to or how long they had to wait initially before being allowed to see their patient. Those who could remember said that they had to wait over an hour and felt this was too long,
“...it was a long time. Over an hour. It was too long. I didn't know
what was going on. We were given no feedback. I just wanted to go in
but we weren't allowed..." (relative 4, female, general unit) 
One relative commented that she had to wait three hours. Another patient was admitted during the quiet time on the unit and the relative was turned away and told to come back at the end of the quiet time,

"...I arrived at 12 noon and was told over the intercom to go away for 2 hours [until the end of the quiet period]. That was very distressing. I was not allowed to see him. No one explained what was happening and why. The reasons were not clear..." (relative 15, female, neurological unit)

Familiar faces

If the patient has an extended stay in critical care relatives become familiar with the nurses. To some this translates into a feeling that they have been on the unit too long. For those who experience shorter stays the number of staff becomes bewildering. Although relatives understood about nursing shifts, they admitted that they would find it comforting to see a regular familiar face. Ward clerks were not enough. It emerged that relatives would find it useful if there was a member of staff who they could turn to with questions about practicalities, such as accommodation, or advice on financial difficulties as a resulting from their family member being in critical care.

“...a member of staff for relatives would be useful for the practicalities like car parking..." (relative 13, male, neurological unit)

Although nurses offered reassurance regarding the patient, relatives did not feel that they were able to offer additional emotional support to them, if they were not coping, primarily because of the assumption that nursing staff had more important things to do. This additional support is something that relatives would have found helpful, 
“...I am surprised that there isn't some sort of counselling nurse whose sole job is just to deal with relatives. A lot of people think that. It would be a really good idea. Someone should be here, especially for relatives on their own..." (relative 3 , female, general unit)

This supports the suggestion put forward in the 1970s (Wallace 1971). However, Wallace suggested that there should be a designated nurse charged with the role of disseminating information about the patient to the relatives, as opposed to the nurse by the bedside. In fact, relatives value contact with the nurse at the bedside and some relatives considered that a separate member of staff to deal with their needs was unrealistic and financially not viable.

"...a member of staff for relatives is unrealistic and we wanted to speak to the nurse [about their patient]..." (relative 12, female, neurological unit)

If a separate member of staff to care for relatives was to be a viable option they would need a different set of skills to those of a nurse, to deal with a separate set of concerns, for example, the practical concerns previously mentioned.

\subsection{Part B: Results and Discussion from Staff Focus Groups}

\subsubsection{Description of sample}

The sample consisted of 26 participants. Twenty were qualified critical care nurses working in either the general or the neurological critical care units at a large London teaching hospital. Six were qualified critical care nurses working at various critical 
care units in Surrey who took part in a pilot focus group. This latter group was considered successful and therefore the data were included in the main study.

\subsubsection{Themes}

Initially the data was analysed inductively in order to establish patterns, themes and categories. To do this, the data was coded line by line through open coding. The codes were refined through axial and selective coding. From this themes and categories were formed by comparing codes for similarities and differences according to their properties and dimensions and grouping them accordingly (Strauss \& Corbin 1998). During the process of coding the data, and as more data became available through continued interviews, the titles and descriptions of concepts were refined. The later generation of hypotheses about the relationships between the concepts involved deductive processes. For a detailed discussion of this see Chapter $3, \mathrm{p} 60$. The themes that were developed fit into seven main categories (see figure 12);

- Information

- Nursing staff

- Doctors

- Relatives

- The environment

- Potential changes

- The staff team 
These categories are comprised of various related concepts e.g. the 'information' category is made up of concepts such as 'information giving' and 'practical information'. Each category and its relating concepts are discussed in detail below.

Preliminary results from interviews with relatives were fed into these focus groups in order to ascertain staff opinion on relatives' perceptions and discuss the practical implications of these views. Nurses continued to discuss and expand upon issues raised in Phase I and Part A regarding the process of giving information and the difficulties they perceive are entailed. Other concepts that developed included staff perceptions of relatives' reactions first raised in Phase I, and the problems nurses encounter when caring for relatives. New issues developed surrounding nurses' perceptions of doctors' responsibilities and the impact they feel doctors actions have upon them. Each of the seven categories above and their related sub concepts are discussed in turn below. 


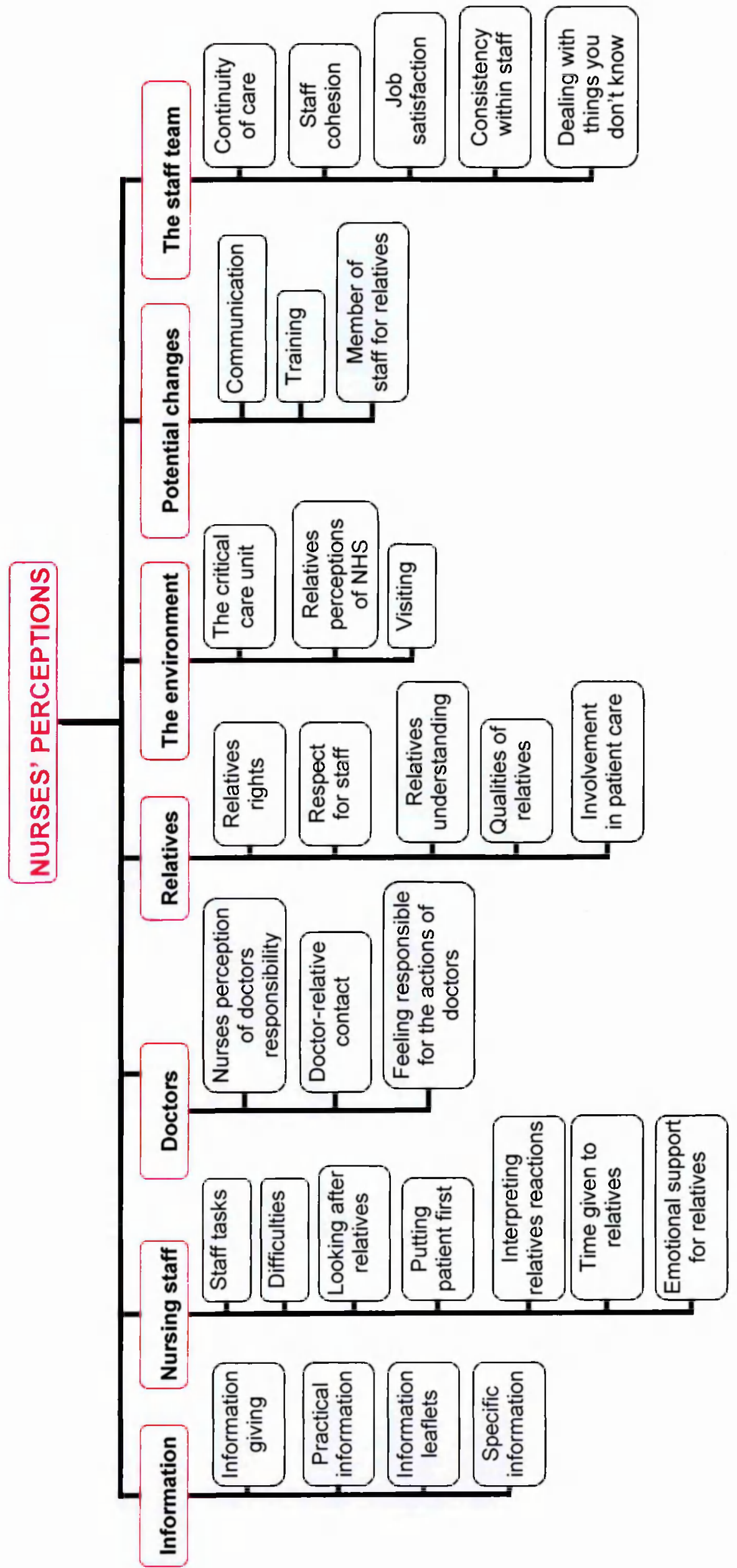

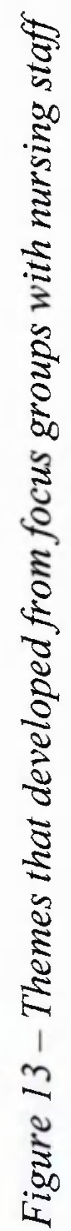




\section{Information}

This category links to the information giving category in Part A. Here nurses discuss the dilemmas they face when trying to give information and why, although they may appreciate what information relatives would like to receive, it is not always possible for them to deliver that.

\section{Information giving}

This concept includes the difficulties nurses experience in giving information to relatives when there has been no change in the patient's condition or when the patients has not progressed as expected. It also includes nurses giving the relatives options and preparing them for what they will face and in the future. In addition, it includes the nurses' dilemma of only being able to give information to the next of kin and tensions arising from this. Ultimately, the nurses are just trying to do their job to the best of their ability in a difficult situation.

When a relative first arrives on a critical care unit, nurses do attempt to prepare them for the situation they are about to face,

"...there is a photo there just outside the door, so we can show them what it may look like when they come in...I normally tell them what they will expect to see, if they can speak to them when they come in, if the patient will be able to speak back. So they don't get really upset. And go from there really..." (nurse 14, general unit)

The aim of this type of preparation is to reduce anxiety. The link between information and anxiety has been discussed previously (see p147). Nurses feel that they can only 
give information to the next of kin and appreciate that this may not be well received by other visitors,

“...it is not always that you don't want to give them information to them before...I tend to only give information to literally the next of kin I don't want to say anything in case they haven't heard before, then you do feel a bit mean saying that you can't give them information would you mind speaking to mum or dad. But I think that is fair enough, because if they were a good enough friend then you would have thought they would be able to..." (nurse 1, neurological unit)

None of the relatives in Part A perceived this as an issue, possibly because only close family members who visited regularly were interviewed.

\section{Information leaflets}

Although nurses acknowledged the benefits of information leaflets, some voiced their reservations. One nurse described how their unit had recently introduced an information leaflet explaining many commonly asked questions, particularly why visiting hours existed, but none of the relatives interviewed from that unit had received a leaflet and few were aware of the reason why visiting hours existed, particularly if they had been transferred from a hospital with different visiting hours,

"...we have a relatives information leaflet that has been developed recently, it explains everything why visiting hours are 2-8 every day of the week Monday to Sunday, everything is there, it explains why. It is just recently been introduced..." (nurse 13 , general unit)

Some nurses felt that information leaflets were not always applicable, 
“...I think a lot of information leaflets you get can be over generalised... a lot of printed information on head injuries is more to do with the rehab, post discharge from an ITU..." (nurse 2, neurological unit)

“...I think also the other thing is people pick up leaflet because they are there and half of them get put in a bin in the corridor..." (nurse 11, neurological unit)

Nurses acknowledge the sensitive nature of giving information, “...I don't know what the answer is on this, you can present people with all the information, you could put it in a booklet in the relatives room, how much they would actually absorb of it and how much would just scare the living crap out of them I don't know..." (nurse 10, neurological unit)

\section{Practical information}

Relatives in Part A did not feel that it was part of the nurse's job to deal with practical issues that arose for the families as a result of their patient being in critical care. Nurses acknowledged that it was their responsibility to inform relatives about the practicalities; however some did admit that they did not always remember. For example, one nurse commented that she often forgot to mention that relatives were entitled to parking permits because she did not drive and it just never occurred to her.

“...I have to admit that is one thing that I never say to relatives. It completely leaves my mind that they can get cheap parking for the week..." (nurse 2, neurological unit) 


\section{Specific information}

In Part A many relatives said that they wished to receive specific information about their patients condition. Nurses do not feel able to give this information for two reasons: firstly, often they do not know themselves and secondly, if they give information and the patient's condition changes or does not progress as expected how they say, they do not want relatives to use that information against them. The nurse below discussed similar problems that she has encountered with communication on a renal ward she had recently worked on,

“...we always used to give transplant information. If something wasn't, not had gone wrong, but had gone slightly differently then they saw it as we had done something wrong, instantly it was because we had done something wrong and that wasn't the case at all. It makes everybody really defensive about things. And that doesn't help anyone. And then they come in with this should have been done..." (nurse 13, general unit)

“...it is alright getting information off the computer on maybe subarrachoid haemorrhage, but that doesn't mean, what's on that information is going to be adaptable to the patients..." (nurse 3, neurological unit)

Nurses found giving specific information about a patient's condition difficult because sometimes even they did not know the outcomes. They also believed that because of the very nature of critical care units, prognoses are very variable and are constantly changing, 
"...Basically what it sounds like is that they really want us to say the outcome of their loved one but we can't give them any guarantees. Even knowing what sort of injury they have got we still can't predict the outcome..." (nurse 3, neurological unit)

Some felt that if, in certain situations, it seemed appropriate to give specific information about a patients condition then nurses should be entitled to do that, “...if you have got laboratory results back or whatever that shows a definite diagnosis or you know exactly what is going on then I think you should basically give information to the best of your ability, if you are a bit unsure you know, ask somebody else, or don't say anything, but if you know what is going on and you feel that it is in the relatives best interests, to be informed..... we are supposed to be autonomous practitioners, so it should be partly our decision as to what information they receive..." (nurse P25, general unit)

\section{Nursing Staff}

This category encompasses issues that affect nursing staff and includes tasks they are required to perform, difficulties they may experience and finding the balance between looking after patients and caring for relatives.

\section{Staff tasks}

This concept involves the tasks and jobs that nurses are required to perform as part of their job. Nurses who worked in the unit with set visiting hours discussed trying to carry out procedures outside of visiting hours, although this was not always possible. 
“...I think with the visiting hours, sometimes it is very difficult. I think most people do try to organise their care and get most of the care done in the mornings. But sometimes there are reasons why we have to get the visitors out i.e. if there is an emergency going on or if they have..." (nurse 14, general unit)

“...in the mornings we do sedation weaning, physio, x-rays. It wouldn't be practical at all..." (nurse 15, general unit)

Feedback given to the staff included the dissatisfaction some relatives expressed about being asked to leave when procedures are performed,

“....and I don't know how well they would take to seeing their loved one having physiotherapy..." (nurse 2, neurological unit)

\section{Difficulties}

This concept includes aspects of the job that nurses find difficult and include dealing with relatives and difficult family dynamics and very large families. Nurses commented that a major problem encountered with relatives is the numbers of people in some families and difficulties in determining what roles people play within that family,

“...when you do not truly have an understanding of who exactly is the immediate family. That can be very difficult. Maybe somebody says they are an aunt and somebody says they are a sister or somebody else will say they are a brother. And that can be very difficult when some other member of the supposed family will say well no that's not it...when you have got conflict within the group as well, that can be 
very, very difficult. Because you end up not knowing whose who in the group..." (nurse 3, neurological unit)

'Difficulties' also refers to nurses' perceptions of problems the face, for example relatives not understanding the rules of the unit and involves issues regarding maintenance of professionalism. Another aspect of the job that nurses find difficult is dealing with relatives' intense emotions,

“...if you get people who have got somebody who is at deaths door and through no fault of there own they are looking for someone to blame and unfortunately your face happens to be in the front line. So we are the ones who cop that initial out pouring of, I guess it is grief really, and be at the receiving end of it and at the same time to think, this person is not meaning to be nasty and aggressive towards me..." (nurse 16, general unit)

Nurses sometimes find it difficult to establish a balance between caring for the relatives and looking after the patient. This is discussed further in the 'putting the patient first' concept.

“...because you are trying to look after the patient and you have to look after them [relatives] as well, that is part of your remit is looking after them as well as the patient. And it can add a lot more stress, particularly if they are very demanding relatives..." (nurse 4, neurological unit)

\section{Looking after relatives}

This concept is concerned with the extent to which nurses look after relatives, including offering reassurance and giving guidance and setting limits. Nurses 
displayed concern for relatives physical well-being of relatives and tried to offer reassurance,

“...you can say go home, go and get some rest. You can explain things

to them. So they are not worried about things. Some people worry about things that aren't worth worrying about, that the doctors aren't concerned about it, but they are really worried about it. So you can just reassurance them because we have seen more patients. They have only ever seen their relative whereas we have seen everyone. And most of them haven't been our relatives. So we aren't going in there with that really emotional viewpoint..." (nurse 14, general unit)

Nurse 17: “...I don’t think sitting beside a bed staring at a monitor hour after hour helps anybody...

Nurse 15: ...I think it is ridiculous. I think people need to be encouraged to get on with the general aspects of their lives. Because it helps them focus on life and yes, their loved one is there, but they are going to be there for quite a few weeks in some cases. I agree definitely..."

(both nurses in same focus group from the general unit)

In Part A, relatives did not always appreciate being forced to go home or leave the unit. They did not perceive that 'getting on with their lives' was appropriate at this point. It is also valuable for patients to have relatives present, as illustrated on p188 in Part A. In addition, encouraging relatives to leave the unit and carry on with their lives does not coincide with the later concept of 'involvement in patient care.' Some nurses believe that relatives should be encouraged to participate in patient care, 
however, this is not possible if they are not present at the unit for lengthy periods of time.

Despite believing that relatives should not concentrate on the monitors nurses still need to acknowledge that they are there and, to some degree, discuss them with relatives. Some nurses believed that appearing not to be worried about alarms and minor changes in the patient's condition was beneficial for relatives,

Nurse 15: “...that is quite good because it makes them think that if the nurse isn't bothered by it then should they be bothered...

Nurse 17: ...I must admit I do tend to say, if I ignore an alarm, I'll just sort of say, it's ok, that it is nothing to worry about. And after that they are generally ok...

Nurse 15:...that's what I say, just tend to reassure them a little bit..." (all nurses in same focus group from the general unit)

Nurses feel that they are in a better position than doctors to talk to relatives and reassure them and because of the degree of contact they have with relatives they are more aware of their feelings and consequently better able to look after them,

“...sometimes nurses can be more tactful because you are with the patient all the time and you are aware more of the patients feelings and relatives feelings and sometimes it can be better coming from a nurse I think, then afterwards you have got the time to gently sit and talk, discuss things, as a doctor is going to be rushing off..." (nurse P25, general unit) 


\section{Putting the patient first}

This concept expands on the 'looking after patient concept' that arose in Phase I (see chapter 4, p113) and includes protecting the patient's dignity. Ultimately, the patient is the critical care nurses priority and there are occasions when other tasks are more important than the relative,

“...you can't help it if you need to do something, especially of the patient just came in and the doctors need to put lines in, so we let them come in for five to ten minutes to be with the patient and then the doctors need to get on with their work. They need these lines more than them visiting their relatives. But we promise them anyway that as soon as we finish we will get them right away..." (nurse 13, general unit)

Nurses also feel very strongly that have to protect their patient's dignity,

"...if the person who is visiting is a man and if the patient is a woman, if it is just a family member not the husband or anything, some people are shy even of their husbands and wouldn't want them to see other parts of their bodies exposed. Me, let me use myself as a patient. I wouldn't want my relative to watch me naked while other things are being done to my body..." (nurse 19, general unit)

"...you have to think of the patient as well...we had a young boy...he was $16 .$. he didn't want to wash and I said well that is fine you can have a wash later on the ward and his mother says oh I'll give you a wash... and I could see a little look of horror cross his face and I had to intervene and said well maybe he wouldn't really appreciate a wash from his mum, no disrespect or anything but he didn't look like the 
type of boy who would appreciate a wash from his mum. So you have to think of those sort of little issues as well and he did agree, he agreed with me. I think he was quite relieved when I said that..." (nurse 3, neurological unit)

Nursing involves a high degree of negotiating access to a patient's body against established social norms (Lawler 1991). Nurses have to balance their experience of contradicting their cultural background with upholding these norms for their patient.

Putting the patient first also involved protecting the dignity of other patients on the unit,

“...the concept as well is confidentiality because their focus is obviously their loved one. They do tend to pick up what is going on in the next bed space that is why primarily they are asked to leave..." (nurse 3, neurological unit)

When asked, some relatives may not want to leave the unit because they do not fully understand the reasons behind the request and thus become suspicious about what is being done to their patient. This may be an example of communication problems between relatives and nurses. In the following quotation the nurse was describing how a friend wanted to come and visit a patient when the nurses on the unit were trying to handing over shifts. On this occasion, the visitor became difficult when the nurse tried to explain to them why they could not visit at the moment,

“... I don't know if he would like you to see him in this condition. And frankly it is no use you coming in and then being asked to go out because we are handing over. We don't want you to hear other patients conditions and if you don't want to agree with me I can not do anything about that but you have to wait..." (nurse 5, neurological unit) 
It is possible that this lack of understanding on the part of the relative related to a lack of appropriate communication.

\section{Interpreting relatives' reactions}

This concept refers to staff perception of relatives and interpreting relatives' emotional reactions and their coping abilities. As shown in Phase I, nurses accurately perceive relatives emotional reactions, particularly on first entering the unit,

"...people who come for the first time, they are going to be shocked whatever happens because their loved one is in the bed..." (nurse 14, general unit)

Nurses also perceive that emotions such as fear, can manifest themselves as anger and were pleased when the feedback they were given was very positive with regard to quality of care and overall satisfaction,

"...often particularly at the door, they are often obviously really stressed and seemingly angry and aggressive, but I think that is just fear sometimes..." (nurse 15, general unit)

Nurses perceive that relatives become fixated on the monitors and feel this is unproductive. They feel that sometimes relatives become more interested in numbers than on visiting their patient and believe that this is a sign that they are not coping very well. This was not something that emerged from the relatives' interviews and illustrates how staff may place their own interpretations on relatives' actions. It may be that the machines and monitors are the only thing that relatives feel are concrete amongst the uncertainty. 
“...sometimes you find that some of the relatives don't actually cope very well and focus more on what you are doing and on the numbers than on visiting their relative..." (nurse 18 , general unit)

Nurses incorrectly perceived that relatives never get used to the environment and feel that when the patient has been on the unit longer than a week, the relatives fear that their patient is going to die. Relatives interviewed in Part A discussed how they did get used to the routine of the unit and their surroundings,

“...I don't think they get used to it. When their patient has been here longer than a week they get frightened that they are going to die or what is going to happen, is that person going to have a quality of life or whatever. I think they are worried..." (nurse 19, general unit)

\section{Time given to relatives}

This concept refers to the amount of time nurses give to relatives and how it affects their workload. Relatives can be both a hindrance and a help to staff who sometimes need a respite from them. Nurses felt that some relatives did not interfere at all with their ability to do their job,

“...some relatives ask their questions, you can feel like you can get on with doing whatever you are doing and you can be talking, especially if their aren't able to talk to their patient because the patient is sedated or something, they are talking to you and you can answer the questions and you can carry on getting on..." (nurse 14, general unit)

“...it is difficult to put it into a percentage of time, because a lot of the time it is like, it is just that they are there and talking to you, but you are doing stuff at the same time. Its not like, if you say how much do 
you spend actually sitting having a conversation probably not that much, but you are just chatting, some of that is about that patient, the information, talking about the condition and introducing long term thinking to them, but a lot of it is just social chatting, but they need that. They need that; they need someone to be normal with them..." (nurse 10, neurological unit)

Nurses considered that generally caring for and involving relatives takes up a lot of their time and may be quite fatiguing,

“...I can understand them wanting to be there because they don't know what is going on and they want to know but sometimes want to you want to get rid of them, if you have got very demanding relatives it doubles your workload..." (nurse 4, neurological unit)

Nurse 17: "...during that visiting time quite a lot of your time...[is taken up with relatives]

Nurse 18: it is quite draining at times..."

(both nurses in same focus group from the general unit)

Despite being exhausting, relatives can be a helpful resource for staff when relatives and staff work together,

“...I think they can be helpful...This patient is not telling you anything and when their relatives come they say I want this I want that. At the moment we have a patient...who speaks very little English. When the relatives come, it helps a lot to be able to express what the patients want..." (nurse 16, general unit) 
“...you also gain a lot of information from relatives, social background and things like that, pieces of history that doesn't always come out when the doctor is there having a short interview and asking regular history, there are things that sort of, you will talk to the relative about, and suddenly they will say something, and oh that's relevant, so if you have not got that interaction with the relatives then you are not going to be getting an awful lot of information..." (nurse P22, general unit)

Nurses occasionally felt that relatives hindered patient care,

“...I think the senior staff probably less so than with the juniors...they find it difficult to get on with their clinical work as well as dealing with relatives, I mean where is the priority? It can be difficult...I mean it is also difficult letting someone taking over with the relatives because then you lose the rapport with them, if you are looking after their son or whatever..." (nurse 2, neurological unit)

Nurses believe that whether relatives were a hindrance or a help varies on the individuals involved. This relates to the concept of 'individuals' in Part A, where relatives felt that staff had individual characteristics which influenced how they felt about particular staff members. Here staff echo this concept about relatives. Any profession that is based human contact will involve individual variance.

\section{Emotional support for relatives}

This concept refers to emotional support for relatives from staff. Nurses perceive that they offer relatives emotional support, 
“...if they want emotional support there is usually the nurse by the bedside, the nurse in charge, or the priest. It is not just one, it is a group effort..." (nurse 13, general unit)

However the relatives in Part A did not perceive that this was always available. Relatives felt that they were given support regarding the patient but not given personal support. Nurses also perceive that relatives build up support networks with other relatives on the unit, although this was not mentioned by relatives in Part A when asked to describe their main means of support.

"... and they talk to each other they build their own network of support..." (nurse 4, neurological unit)

Some nurses felt they were unable to offer emotional support to relatives because of their own characteristics,

“...depends on the situation, and it depends on the relatives and also it depends on the nurse. Some of us are much more open and much more able to deal with some of the emotional upset than others. If you are shy yourself and guarded about your private life then it is not so easy...you almost feel sometimes that you shouldn't be too emotional. Because you have to be the support for them you can't cry bucket loads with them as well. I think sometimes you do. I know some staff who have got more involved with relatives than others and it can cause problems..." (nurse 4, neurological unit)

In the next quotation this nurse expanded on what problems she felt could result from giving too much emotional support to relatives. She believed that there was a danger of overstepping professional boundaries and becoming too involved, 
“...you could get to a situation where you get very involved with relatives on a very personal level and you haven't kept that sort of professional relationship. You could get into a situation where they are looking to you to change things, they may be looking to you in desperation or hope that something, you promised it would be alright and blaming that person, so that that person could then end up feeling very bad about it...we did have somebody who was very long term...and somebody did get very involved and it was difficult then when things were going to take a turn for the worse, because she felt bad and...she found it hard to deal with and her own personal emotional experience. And then I think sometimes if you get very, very too involved if the person isn't looking after that patient for whatever reason then the relative questions why...it can potentially lead to difficulties..." (nurse 4, neurological unit)

This relates to the concept of emotional labour discussed in the literature review (see Chapter 2 p26) and whether caring is something that can be learnt or whether it is an inherent characteristic. Smith (1992) believes that emotional labour required for nursing can be taught and necessitates formal and systematic training to manage feelings.

\section{Doctors}

This category encompasses nurses' perceptions of doctors' responsibility and involvement. 
Nurses perception of doctors' responsibility

Many nurses feel that ultimately it is the doctors' responsibility to talk to the relatives about their patient's condition. In Phase I, although relatives perceived nurses as competent they still wanted information from doctors. If nurses also feel that doctors should give information to relatives it will encourage this perception.

"...I think that is up to the doctors to do that [talk to the relatives]. They are the ones who are really in charge of the patients and they are the people who should break bad news to the relatives. And if the relatives do not understand things and want a simpler explanation to what they have heard then we can help them. But I think it is something the doctors should deal with..." (nurse 19, general unit)

Nurses acknowledge that they sometimes refer relatives to doctors if they do not know what to tell relatives despite recognising that the doctors may not know any more than themselves about the patient's condition,

“...sometimes doctors have never seen the patient before, especially if they have been off for a week. But they have to come and talk to them...obviously they know the doctors aren't here all the time. They might just be able to present a good story. In a way, no one is harmed by that..." (nurse 14, general unit)

Some nurses feel that doctors try to avoid their responsibilities to relatives, “...doctors try and get out of talking to relatives..." (nurse 11, neurological unit)

In addition, some think that nurses need to know their own limitations of what they are able to do, 
“...you have to know the limitation of nurses, you have to know how far you can actually inform relatives of what is happening...so you should have the same statement that everybody says up to this and that is it and if it is something else, then just call the doctor..." (nurse P21, general unit)

The quote above illustrates that one way to improve consistency is for all information to relatives to come from doctors. An alternative argument is that this merely transfers responsibility. Doctors have the potential to be equally as inconsistent as nurses.

\section{Doctor-relative contact}

The most usual contact between doctors and relatives occurs when relatives ask to see a doctor,

"...sometimes it is when the relatives ask. That is mainly the time when they will see the doctors or when they do their rounds in the afternoon. Or when they are really sick and doctors want to prepare them for what is going to happen in the future. Not routinely. If relatives ask they will speak to them..." (nurse 13, general unit) "...if they happen to be around when the consultants are doing their rounds, then they generally have a chat. Otherwise...Most of them do ask, if they haven't seen anyone, especially in the initial phase. But if it is at a time when there is just the on call person, they don't generally have the specifics anyway, the surgeons are pretty good, if you call them then they will come and have a chat..." (nurse 2, neurological unit) 
Nurses feel that it would benefit relatives and relieve the added pressure on them if doctors routinely saw relatives for a short period each day or two, even if the patient's condition is unchanged. Currently, doctors do not routinely see relatives,

Nurse 10: “...I would like to create an expectation that a surgeon will speak to a family member once a day. The problem is that you say to the doctor they want to talk to you and the doctor will say but nothing has changed, and you say that they need to hear that from you, they want to hear that from you. They don't get that. The surgeons only think they need to talk to someone when there is a major development of some sort. I would like to have a protocol...

Nurse 11: ....a set time...

Nurse 10: ...yeah, that they came back from the ward round and did it...I'd say nearly all the time I am happy. Occasionally things don't go well. I would say the most common reason for things not going well is the doctors' resistance to speaking to them..."

(both nurses in same focus group from the neurological unit)

This reinforces the earlier argument from Phase I that indicated that despite nurses having the most contact with relatives, the latter still want to receive information from doctors. This expectation may have arisen from the media portrayal of hospitals in television dramas, as discussed in Phase I. In addition, nurses feel that relatives are more content once they have spoken to a doctor and thus nurses find them easier to care for,

"... when they have spoken to the doctors anyway they feel happier.

Sometimes they are ok with the nurses, but they tend to want more 
information, so they tend to get that information off doctors and then they are happier..." (nurse 13, general unit)

However, despite acknowledging that relatives are happier once they have spoken to doctors, doctors do not speak to them very often.

\section{Feeling responsible for the actions of doctors}

Nurses interact with relatives on a daily basis, whereas doctors do not and as a result nurses feel responsible for the actions of the doctors. They feel guilty if a doctor says that he will do something at a certain time and then does not. Nurses feel that they have let the relative down, even though it was not their fault or responsibility,

Nurse 10: “...when they [doctor] say I will come and see them after the round and they don't. That can be very, very difficult, that is the source of considerable stress for nurses, because the nurses are the ones standing there feeling the eyes burning into you if you like, feeling the failure. You just feel so uncomfortable when a commitment has been made and has not been met. I really find that difficult. I wouldn't say we get blamed, but you just feel guilty, you feel responsible..."

Nurse 11: ...I usually explain that we have asked. And just try to explain why they haven't come to the best of your ability..."

(both nurses in same focus group from the neurological unit)

“...that is ok until you get situations where doctors haven't spoken to relatives and you keep saying to them...that somebody needs to speak to this family and they keep saying yes, yes they will we are busy, and 
four days down the road you have still got relatives who are asking what has been shown on specimens that that been taken and you know...in those circumstances I think you just sort of give them a little bit more... if doctors do tell the relatives something, it is always the nurse that, the doctor will just leave and you are the ones left just picking up the pieces and trying to fill in the gaps.." (pilot study, nurse indistinguishable)

\section{Relatives}

The concept encompasses relatives' rights, respect for staff and understanding. It includes perceptions of relatives' qualities and the involvement of have and involving relatives in patient care.

\section{Relatives' rights}

Nurses felt that in recent years attitudes in society had changed and relatives were more aware of their rights than ever before,

"...even if you say, you are there to help your husband, father or whatever they don't see it like that they see it as their right to come and visit whatever time they want to and you ask them to leave at 8 o'clock. No. So much that you have to get security and it does happen and it has happened and it is happening quite regularly. I think it is just this aggression that people have in culture today..." (nurse 15, general unit) 
“...much more demanding, much more aggressive. Much more inclined to say, it is my right, that is something I really have noticed..." (nurse 17, general unit)

Although nurses recognise that relatives have a right to visit their patient, there is a balance to achieve between relatives rights and their priority, which is to make the patient better.

Respect for staff

Nurses feel that some relatives have less respect for the nursing profession that they used to and this makes their job more difficult. As with the previous concept of relatives' rights, nurses feel this is a societal change,

“... and not frightened to kind of, they used to treat you with a certain amount of respect, now I don't feel that, as you say a good number of people don't have any self respect for you. And that is definitely something I have noticed..." (nurse 17, general unit)

Nurses feel that a sign of diminishing respect is the increased incidence of aggressive behaviour. Nurses accept that there is a chance that the intense nature of critical care units is more likely to provoke aggressive behaviour that on a general ward, "...the relatives we do get up here, if they are going to be aggressive, they do tend to be quite aggressive and maybe not necessarily would be like that on the ward. They would be dealing with whoever is in charge rather than necessarily the junior nurse at the bed. Here the nurse at the bed is the face of the unit..." (nurse 16, general unit) 
As mentioned in 'interpreting relatives' reaction' concept nurses recognise that displaced feelings of fear can manifest itself as anger. Nurses also commented that the lack of respect that they feel exists could be subject to regional variation,

“...it definitely seems to be a London thing. I have worked a lot out of London; relatives outside London do not talk to you like that. The difference is unbelievable. They really respect you, it is just incredible the difference. They see you like, I am not saying we should be put on pedestals, but a little bit of respect for our job, and that we are trying to help their relative at the end of the day..." (nurse 15, general unit)

\section{Relatives understanding}

The extent to which staff perceive that relatives understand what is happening and what they have been told is a concept that emerged in Phase I and in Part A. In Part A relatives stated they understood everything they had been told. However, when asked what they had been told in the initial phase many relatives could not remember. Nurses perceive accurately that relatives do not always absorb what they are being told. This reinforces much of the literature regarding absorption of information in a crisis discussed previously (see Chapter 2, p20),

"... it just goes in one ear and out the other, and it doesn't matter how many times they have been told they still don't get it..." (nurse 18, general unit)

"...we calm them down and are gentle with them. Everyone has to be gentle with them. They try to understand a few things but you can see that, they ask you the same things, that is why I say that they can see you talking but they don't hear you..." (nurse 19, general unit) 
“....if you are actually sitting there listening to what the doctors was saying you have some idea of the information that they are meant to know as oppose to them getting confused they think the doctor has said one thing when they have said something completely different and if you weren't in the room then you are not going to know...and then you explain again and again and again until they get it..." (nurse P25, general unit)

Some nurses felt that lack of understanding by relatives was because they did not possess the same medical background that the nurses had,

“...they don't understand, they haven't studied medicine, they have no idea what is in the body..." (nurse P21, general unit)

\section{Qualities of relatives}

Nurses discussed the positive and negative qualities of relatives that ultimately make their job easier or more difficult. Qualities in relatives that nurses perceived as positive were an appreciation of the jobs that nurses do, a trust in what nurses are doing, asking questions, interacting with the patient by talking to them and touching them, behaving responsibly on the unit, adapting to the environment and trying to stay calm. Nurses felt it helped if relatives had a good support network behind them.

“...you get all sorts of relatives, with different sort of personalities. And there are some who appreciate what we do and there are some who just don't..." (nurse, 1, general unit)

"...a good visitor is someone who is just here to visit their relative and quite happy to hold their hand and talk to their patient and still interact with me and ask me questions and things like that but not jump on you 
about every little thing. It is hard to give examples about that..." (nurse 18, general unit)

“...you need to feel they trust you and trust what you are trying to do with the patient and believe what you are trying to do for the patient..." (nurse 4, neurological)

“...adaptable to the environment. Has a bit of an understanding. Knows the boundaries basically as well..." (nurse 3, neurological unit)

Perceived negative qualities were being particularly demanding and questioning whether what nurses were doing was best for the patient, being aggressive, manipulative or not abiding by rules on the unit, being disrespectful or unappreciative.

"...people that play one nurse off against each other..." (nurse 17, general unit)

"...I would call a very bad relative someone who is aggressive..." (nurse 19, general unit)

There is extensive literature on what makes certain patients popular and other not. This could equally be applied to relatives, although there does not appear to be any published research with this application. There is evidence to suggest that within health care settings people interact in a qualitatively different way with clients/ patients depending on their view of them (Johnson \& Webb 1995). Treating all patients and relatives equally irrespective of judgements that have been made about them is a challenge for emotional labour in nursing.

\section{Involvement in patient care}

This concept emerged in Phase I where relatives were keen to be involved in patient care. It was not an area discussed by the relatives in Part A of this study. However, it 
was apparent from nurses' comments that some relatives were more willing to become involved in patient care than others. Many nurses believe that being involved in patient care should be encouraged. Staff in Phase I acknowledged that they felt relatives were an underused resource.

“...initially it is probably quite time consuming because you have to you do things in a specific way but once they actually, once they actually know what they are doing it can actually probably be quite a great benefit to, you are aiming to provide eye mouth care roughly $1-2$ hourly depending on the type of patient that you have got. So each time that is taking you between 5 and 10 minutes to do that, so if you have got someone there doing it for you then it is a benefit in the long term, it frees you up to do other things that obviously they can't do. If they do take part in basic care I think it is of value..." (nurse P25, general unit)

“...I think relatives should be encouraged to get themselves involved in the care of the patient, like mouth care and applying moisturiser their feet and hands. That should be left to them to do, but some people think that it is your job and you have to do everything. It is nobody's job to do be doing those things, application of moisturiser, because you have got other important things to do. So I think if they all cooperate...we don't really mind, so far as it doesn't interrupt with any other things we do. Many people do..." (nurse 19, general unit)

Some relatives do get involved, "...I have seen one lady but she was a nurse, so she used to help up with washing and rolling...she was helping all the time, changing sheets, washing. 
She was really good. They are nervous because the patient is on all these machines, so they are nervous..." (nurse 20, general unit)

Other relatives do not want to be involved at all and this surprises some of the nurses,

"... [the patient] been here more than two months now and I have seen the wife and she is not really doing much interaction with the patient...and I was wondering if the wife would be eager to participate in simple care but she is very hesitant and I have asked her is this ok, have you done this before for your husband, things like that and she is just very blunt, she is wondering why I am asking her about that...she just laughs and stares at me and I don't know whether she is able to understand what I am trying to imply here...I was a bit shocked with her reaction... and she was a bit shocked because I asked her whether she wanted to participate in some of the things..." (nurse 5, neurological unit)

“...I think it all depends on the living condition at home. Because some people have a nonchalant attitude towards their partners, or their husband or wives and if that doesn't really come from home, there is no way you can start that off because the person is ill. That common sharing of some women who take the joy of cutting their husbands toenails and when the patient is also sick, you also want to be doing those things....or bathing together or doing things like that..." (nurse 6, neurological unit)

This nurse illustrated her point with the following example, “...she requested to wash him...and we washed together so when it came to rolling I had to get someone else and she said I can roll and I 
said ok you can roll you can help us and give us a hand but you aren't covered by the trust you're not insured in case you break your back or anything happens to you and she gave the man a very nice shave and a wash, did his perineum area and everything and that was really, really brilliant. And it appears she must have been doing at home because it's not something that you just come out today and just all of a sudden start doing it....no....it is a habit that is cultivated at home before you come here..." (nurse 6, neurological unit)

The nurse highlighted the insurance implications of allowing relatives to participate in patient care and its dependency on the patient's condition, “...we wouldn't allow that to happen all the time...it depends on the patients condition, and sometimes it depends, we could allow that to happen because the relative would get some psychological satisfaction from doing that...she was really, really satisfied after washing her husband..." (nurse 6, neurological unit)

There was an opinion that perhaps the involvement of the relative in patient care or not was related to communication issues,

"I think that is an interesting point because most people you see you feel like they don't want to get involved maybe the lack of communication between yourself and the patient's family and other times are just so hectic that you haven't got the time to be saying oh do you want to do this..." (nurse P26, general unit)

Nurses felt that involving relatives in patient care was a way to make them feel useful, 
“...it gives relatives something to do as well, the amount of time I have been doing things and they say it is so frustrating just sitting here, I wish there was something I could do erm, I know when I have said you can help...brush their hair clean there teeth. In my experience they have said oh no...some people are quite open to the fact of doing things for others....husband and wife you know, you wouldn't think anything of brushing their teeth, somebody else might see that as intrusive if they are not together, people that have done things for others, for their relative, erm, they seem a lot more calmer, a lot more relaxed...they not enjoy doing it but it gives them reason to be there instead of just sort of sat there doing nothing..." (nurse P24, general unit)

It has been shown that good relationships between relatives and nurses can be created when relatives become involved in certain aspects of patient care, for example, eye and mouth care (Hammond 1995).

Much of the involvement in patient care relates to the concept of putting the patient first and protecting the patient's dignity. Under certain circumstances it may not be appropriate to encourage a visitor to undertake personal care.

\section{The environment}

This concept refers to the critical care environment and includes perceptions of the unit itself and staff opinions on relatives' perceptions of the NHS in general and visiting policies. 


\section{The critical care unit}

Nurses acknowledge the threatening nature of the unit and remember their reactions when they first experienced a critical care unit. This also relates to nurses accurate perceptions of the emotions relatives experience when they first enter a critical care unit see p181 and Chapter 4, p109.

“...when they first visit everything is scary and we take it for granted because we are so blasé, we take it for granted. We have just got used to it..." (nurse 15, general unit)

“... I remember the first time I walked into intensive care, I went to visit a friend, I was in my nursing training but I hadn't been into intensive care. I was just completely and utterly flawed by it...so I can't imagine anybody who has never had any kind of medical exposure walking into here, it must be like walking into hell..." (nurse 18 , general unit)

One nurse described how her boyfriend's father had recently had a heart attack and as she did not know him well she was not very emotionally involved in the situation but played a supportive role. She thinks that this experience has changed the way she will perceive relatives in the future,

“...it was very interesting like, I gave them the hope and I involved them in what to do, bring all his pictures, make it look like a home, and other things they wouldn't even think about, like they didn't know what to do, they were scared to ask staff, what is it on the monitor...I just told them, you have to ask things, you have to ask them to explain to you...but I was looking at it and that is happening every day and 
people they do not ask, they did not ask at all, but because I was there and I was trying to explain, to calm them down...now I think I am going to have a different view on relatives, because if it doesn't happen to you personally you just think differently, you just think, oh, I don't want to have them there when I do something, I think it will change your opinion when it happens to you..." (nurse P21, general unit)

\section{Relatives perceptions of the NHS}

This concept mirrors the 'relatives' concept that critical care was 'different to what was expected.' Nurses perceive that relatives have an unfounded negative perception of the NHS in general, but when they have to access services they are pleasantly surprised,

“...it is nice to hear that people are generally positive. I think that reflects what most people say about the NHS, even if people go to out patients I have heard so many people say this, they say they had a really good service, and everything is so much better than it used to be but they assume that that is not typical of the rest of the service. They had a good time, they had a good service, but no one else gets one. The whole thing is in crisis, but actually when I saw the consultant, he was really nice and blah, blah, blah, I think people have got a really weird perception of the service at the moment. In many ways it is a lot better than it has been..." (nurse 10, neurological unit)

It also relates to the theme in Phase I that relatives gain perceptions of critical care through the media. 


\section{Visiting}

Nurses from the general unit discussed the reasons why visiting was only in the afternoon. However in Part A (p 187) relatives were dissatisfied with the limited visiting hours. Nurses said they told relatives the reasons for restricted visiting but relatives appeared to either be unaware of these reasons, or simply may not have absorbed them when told,

"...visiting is only in the afternoon, because it is so busy in the morning and they would be leaving all the time. There are physios...it is really busy, all the rounds, the doctors..." (nurse14, general unit) “...I think with the visiting hours, sometimes it is very difficult. I think most people do try to organise their care and get most of the care done in the mornings. But sometimes there are reasons why we have to get the visitors out..." (nurse 15, general unit)

Another reason why quiet time and visiting hours are imposed is for the benefit of the patients,

“...it is tiring for the patients..." (nurse 18, general unit)

As mentioned previously patients often want the relatives there and notice when they are not there, see Part A, p188.

\section{Potential Changes}

This concept includes ideas that staff proposed for potential changes or improvements to existing practices. Issues surrounding communication, training and additional roles were discussed. 


\section{Communication}

Suggestions were made for access points to enable relatives to gain further information on their patient's condition. However, the feasibility of this was questioned,

“...we couldn't give them a computer or anything, because it probably wouldn't be used for that, it would be used for....you know not anything bad, but...it would be nice to have an information point..." (nurse 1, neurological unit)

Ways in which staff could make practical information more explicit was also discussed. Ideas such as laminating the relevant forms (e.g. for a parking permit or accommodation application) and displaying them in the waiting rooms to prompt relatives to make enquiries were suggested.

Knowing what information relatives had been given by doctors was important to nurses. Although much of the time nurses are present during discussions, sometimes this was not always possible and in those situations, the current methods of discovering what relatives had been told was considered inadequate,

"...communication wise....it gets forgotten...on the medical notes there is a section where the doctor writes relatives notes about what he told the family, half the writing you can't read [laughs] and talking to them...how much do they know, what information they have been given before...you need to know where you are going and how far you can go, I mean, you have certain things you have to leave to doctors, you know, that is why they are there..." (nurse P24, general unit) 
Other issues relating to communication such as, nurses views that doctors should speak to relatives on a regular basis (p215) and relatives perceived confusion over visiting and being asked to leave the unit (p187) have been documented earlier in this study and have been discussed at the appropriate point.

\section{Training}

Nurses commented that they do not receive specific training on critical care situations and that any additional training which is available is too general. Critical care is very different from many other units in the hospital and it is felt that tailored training is required.

“...people with more experience learn to let it go and just agree with them [relatives], or not agree, but just say I understand and be understanding, whereas a junior member of staff may not be able to handle that because they haven't got the experience and maturity and experience to do so. There is training for aggressive patients and relatives but ITU is so different...maybe we should put that forward to the D grade programme..." (nurse 15, general unit)

Nurses commented that they do not receive any training on handling difficult situations,

“...what we do if we do have a really difficult set of relatives we have a staff counsellor...more often that not none of us want to go to her sessions because we are all quite happy thank you very much. If we have a really, really bad set of relatives or we have a situation which is very sad and has a lot of ethical and moral issues around what is going on with the patient, we sit down in a group and she goes through how 
we feel about it. Because we have had some very difficult relatives in the past who, some of them have been very manipulative as well and that is another thing...they compare one nurse against another and say I don't want her, I don't like her, I don't want her, I only want her to look after him because I know she will take care of him. She is very good in those situations..." (nurse 4, neurological unit)

Within the Hospital Trust training, such as dealing with difficult situations or dealing with aggression, was available to nurses at all levels however, many did not know that it existed and those that did assumed it was for higher grade nurses only,

Nurse 3: "...I did it as part of my management course at another trust, and actually it was very positive. Because I have been in situations where I have had very angry aggressive people and I know how to deal with it. I don't always get good results. But at the same time I know when to back off know now, you know, to other members of staff as well. I certainly learnt a lot about how to control my emotions...

Nurse 1: ...does that come as more of a management thing, it seems to, when you do management training you seem to get assertiveness training?

Nurse 2: ...there are study days available within the trust, dealing with difficult situations and that kind of thing, so they are available...

Nurse 1 ...for every grade?

Nurse 2: ...yeah..."

(all nurses in same focus group from the neurological unit) 
The staff counsellor referred to earlier, was actually no longer operating in connection with that unit, this was considered a loss by the staff, "...the nurse counsellor used to do training specifically for this service, dealing with difficult situations and difficult people, but that has stopped because she has too many things on...the teaching on the whole, has never been about how to actually do it, it is more about the impact is has on you. That was certainly the nurse counsellors approach, was more about how it made you feel to deal with these things, not about the strategies for initiating conversations, the sort of thing they teach in the medical school. I think the assumption is, and probably a correct assumption by and large that that sort of thing is covered in people's training. Maybe I am wrong. Some of the surgeons could do with it..." (nurse 10, neurological unit)

Some nurses felt much was common sense and experience and not skills that could be taught,

“...A lot of it is just common sense. I don't think it can be taught. It would be very difficult..." (nurse 11, neurological unit)

This is related to the concept of emotional labour discussed earlier (p213) and whether it is a notion that can be taught. Nurses in Smith's study (1992) felt that they learnt to detach themselves emotionally through trial and error. However, they are able to learn to manage their emotions in certain situations through intensive training in deep and surface acting (Hochschild 1983) (see Chapter 2, p26). It was found that more experienced workers were particularly adept at deep acting, which enabled them to 
make a distinction between their 'personal selves' and their 'work selves', developing a healthy separation which in turn helped to prevent burnout (Smith 1992).

\section{Member of staff for relatives}

Nurses discussed the benefits and drawbacks of additional member of staff with a pastoral role on the unit to care for relatives. The following nurse used an example of from a previous unit she had worked on,

“...I came from the renal unit and we had a social worker, who was also a trained counsellor. She would see relatives as well as seeing patients and people who go to her and relatives would ask to see her. She helped with a lot of things, because she was a social worker as well she helped with benefit forms and all that sort of thing and these changes that would happen, from that point of view she was really, really good and we enjoyed her coming around and she would give us advice on what to say. And we had the chaplains coming round. We had a dedicated chaplain to the unit. He was useful in a non religious way as well..." (nurse 14, general unit)

“...that would be nice, but the problem is where we would get the money..." (Nurse 14, general unit)

The neurological unit in this study did have a social worker that staff could telephone if necessary, but more junior members of staff did not know that this resource was available.

“...like that young Australian lad, they came over here and they aren't the wealthiest of families and the other week I said have you spoken social worker, and they hadn't and I got the social worker to speak to 
them and that was another avenue to help them try and get him back to Australia, which has been their main concern. There is a social worker attached to the unit. She is at the end of the phone, she is very good..." (nurse 11, neurological unit)

They also mentioned a staff counsellor who was previously attached to the unit and who occasionally would talk to relatives, although it was not strictly part of her remit,

Nurse 4: "...her remit is not really talking to relatives, but sometimes she does offer. I think doesn't she...

Nurse 5: ...I have asked her once before. We got one patient where the family asked if there is anyone they could talk to because the children were little ones and I don't know how to tell them what has happened to their dad. I said I'll ask some one, it's not really, but she is linking with the staff, but she was happy to talk to them. It was asking a favour..."

(both nurses in same focus group from the neurological unit)

Other nurses did not believe that having a member of staff to care for relatives was a good idea because they considered it part of their job to care for relatives, although they could see the benefits during certain phases of care,

"...you are used to the relatives and they trust you, so it is like a two way process, whereas if you had somebody just to oversee the process so it would be like taking a part of your job away really which would be quite sad..." (nurse P23, general unit)

“...I can see that working when initially a patient comes in and you're busy with the patient and say ....you might have relatives waiting in 
the waiting room for an hour and a half, two hours, and no one has spoken to them...I think it could be useful that way to bridge a gap, until you can go in to speak them or the doctors can go and speak to them, because a lot of the time they can be left in the waiting room for a long time...you can't leave the patient because the patient is the most important at that time, so that's quite helpful..." (nurse P24, general unit)

This also reiterates the argument that the patient is the priority and an additional member of staff to liaise with relatives would ultimately improve patient care.

\section{The Staff Team}

This concept refers to how the staff on the unit work together, how they deal with things when they do not know what to say and the achievement of job satisfaction.

\section{Continuity of care}

Relatives in Part A and in Phase I valued continuity of care and appreciated seeing the same faces. Nurses also acknowledged the importance of this,

$$
\begin{aligned}
& \text { "...it does help that the more you work with the same patient and get } \\
& \text { to know the family they get to know you obviously and understand } \\
& \text { that you are quite busy and don't have to keep asking the same } \\
& \text { questions..." (nurse 1, neurological unit) }
\end{aligned}
$$

Staff in Phase I discussed is more detail how hard it is to achieve that continuity (see Chapter 4, p 117). 


\section{Staff cohesion}

The nurses on both units felt that they worked well as a team. This was reflected in the relatives' perceptions in Part A, p170.

“...we are lucky here because as soon as there is a situation you know you have got a lot of support and things..." (Nurse I, neurological unit)

This obviously varies greatly, depending on the unit, management and individual team members.

\section{Job satisfaction}

Nurses acknowledge that they obtain their job satisfaction from helping people. They also feel that relatives are less respectful than they used to be (see p218) and this diminishes their job satisfaction,

"...we are not here to harm anybody. We are here to look after them and to make sure they get well and when they get well that is when we get our job satisfaction. We don't get it from anywhere else..." (nurse 19, general unit)

“...they are very grateful and it is very good actually, you feel like you are needed at that time. It feels good..." (nurse 13, general unit)

\section{Consistency within staff}

Staff admitted that sometimes they were at fault when they were inconsistent within the team, for example about giving information over the telephone, or letting a relative visit five minutes early or stay late. These practices caused problems within 
the team, because once one nurse had set a precedent the relative assumed it should be like that all the time,

"...that nurse did it that way and they allowed me in at five to two, so expect you to, this sort of thing. But that is us, we need to be consistent about what we do..." (nurse 15, general unit)

This elicited agreement within that focus group. A similar example arose in a focus group with the neurological unit where, on one occasion a relative had been given too much information over the telephone and was now causing problems when they the same level of information was not forthcoming every time,

Nurse 11: “...That wasn't their [relatives] fault that was down to the nurses because they shouldn't have been given so much information over the phone...

Nurse 10: ...it's down to the doctors as well...

Nurse 11: ... once you have done that once it is very hard to back on it..." (both nurses in same focus group from the neurological unit)

This also illustrates the contribution doctors make to the level of consistency in information received by relatives. It is nurses as the face of the units that have to deal with the consequences of inconsistency by doctors.

\section{Dealing with things nurses do not know}

Nurses acknowledged the importance of confidence and being self assured. They felt that if relatives surmised they were not capable or knowledgeable, they would not be confident in the care their patient was receiving. They believed that by sounding sure even if that were not the case would enhance relations with relatives, 
“...I would never just say I don't know just as 'I don't know'...it is worse to say an 'if' or a 'maybe', because then you might give the wrong information. It is really important you have the right information, it is a really valid question and I don't want to give you any false information, let me find out...I don't want you going away and worrying about something when maybe it's not be a problem at all. Normally, if you are quite assertive in yourself, I do know what is going on and I am going to sort it out. Rather than being all I don't know. They see that then and they get worried, if you are not confident with them. They are not going to be confident in the care you are providing..." (nurse, 14, general unit)

"...if you are not too sure what is going on then don't say anything..."(nurse P25, general unit)

Some nurses chose to deal with situations where they did not know the answer by passing the responsibility to the doctors. Nurses acknowledged that sometimes this was a way out,

“...sometimes if I am not sure of the answer I will say I will get one of the doctors to have a word. Or if they feel that you don't know what you are talking about they usually ask anyway can I see one of the doctors. Doctors are made a scapegoat..."(nurse 13, general unit)

This relates to the previous concept of nurses perception of doctors responsibility (p213). Doctors are perceived to have authority and are responsible for their patient. Nurses realise that they also have a responsibility to the relatives to keep them 
informed. Referring information-giving to doctors is perceived to keep relatives happy but also divests nurses of responsibility for that information.

\subsection{Mismatches between relatives and staff perceptions}

There were a number of occasions in which staff and relatives' perceptions differed. These mismatches predominantly occurred around areas of communication and concern for relatives' well being. Relatives wished to receive specific written information about their patient's condition and the potential outcomes. Staff understood why relatives' wanted this but believed that they were not in a position to offer this type of information either because they often did not know the outcome or feared litigation if the situation did not progress in exactly the way they had speculated. In addition, nurses perceived that giving information only to the next of kin could prove problematic for relatives and other visitors but relatives did not raise this as an issue of concern at all. Relatives did not consider that it was the nurses' job to deal with practicalities such as parking, when nurses acknowledged that this was certainly part of their remit.

Staff showed concern for relatives' physical well being, for example, by encouraging relatives to leave the unit and get rest. Often this was not looked upon favourably by many relatives who wanted to be near their patient. Staff believe that they are currently giving relatives emotional support but relatives do not feel that they are receiving it. Nurses incorrectly perceived that relatives never get used to the critical care environment but relatives discussed how they became used to the routine of unit. This mismatch may be due to a misperception over the nature of support.

The mismatch between staff perceiving that they already offer this support and relatives' awareness that it is lacking, highlights how staff may be prevented from 
embarking in the emotional side of relatives' care because of the issues that can be interpreted within a framework of emotional labour (Smith 1992). Nurses use a variety of mechanisms which allow them to engage in an essentially demanding and stressful profession while at the same time regulating and managing their own emotions. They do this with varying degrees of success.

The need to manage emotions is implicit within nursing practice but is rarely explicitly addressed (Smith 1992). When not explicitly addressed the emotional labour inherent in nursing may impede their ability to address the emotional needs of patients and their relatives.

\subsection{Limitations}

Nurses have the most interactions with relatives, so it was deemed appropriate to only involve nurses in the focus groups. However, in future research it would be useful to gain input from other medical professionals who worked on the ward. A further possible limitation is that the relatives who chose to participate in the study may have been those who were coping better with their experiences; this was unavoidable taking into account ethical considerations.

\subsection{Conclusions}

Overall relatives were content with their experiences of critical care. There appeared to be little differences in perceptions between relatives of planned and unplanned admissions, this supports existing literature (Twibell 1998). Those relatives with previous medical or critical care experience felt better equipped to deal with the situation. There also appeared to be little difference between the reactions of relatives whose patients were admitted to a specialist neurological critical care unit and a 
general adult critical care unit. However, issues did arise concerning the type, nature and process by which relatives received information from doctors and nurses on critical care units.

Relatives wanted to be kept informed of all stages of their patient's condition and received a mixture of factual information and reassurance which they found helpful. They also found that information they received from staff was consistent and comprehensive and they valued this highly. Consistency of information was an issue that emerged in Phase I as problematic; however, it did not arise for this phase. Relatives were aware that staff could not change the situation but nonetheless felt that they needed something to hope for and did not appreciate always receiving information in a negative manner. The importance of hope in effective coping has implications for medical staff interactions with family members of critically ill patients. When communicating with relatives, doctors and nurses need to be aware of statements that indicate hope or lack of it as this may influence relatives' ability to cope with the situation. Relatives observed that the quantity of information they received varied depending on how confident they perceived the nurse at the bedside to be in his or her own abilities. Nurses agreed with this perception and felt that if they did not appear confident then relatives would not have faith in the care that their patient was receiving and would not trust the information they were imparting.

As in Phase I, relatives contact with doctors was limited and appeared to occur by chance if relatives were at the units when the doctors visited. Relatives differed in opinion as to whether they were satisfied with this; satisfaction seemed to depend on how many chance meetings had occurred. Figure 13 illustrates relatives' (next of kin) interactions within critical care units. The solid lines illustrate frequent contact and the broken line represents limited contact. 


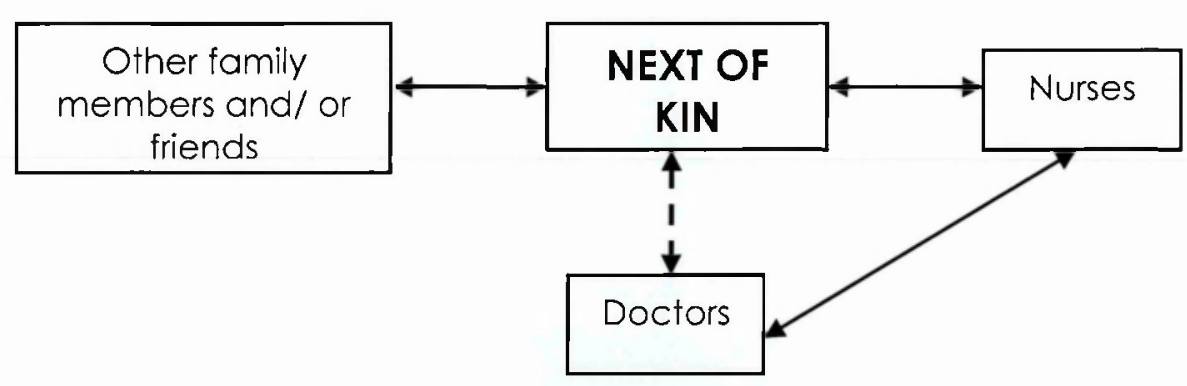

Figure 14 - Relatives' interactions within critical care

Relatives also felt that doctors frequently did not introduce themselves at the bedside and consequently they were unclear as to which staff were doctors reflecting a further communication issue. Relatives are more content when they have spoken to a doctor; nurses also perceive this and as a consequence find relatives easier to care for. This is not something that occurs as a matter of course and nurses would like to see doctors routinely talk to relatives even if there is no change in the patient's condition. This expectation of contact with doctors may have arisen from media portrayal of hospitals and critical care highlighted in Phase I. Possible solutions for this problem may be to inform relatives that staff are aware of potential expectations however, those expectations are not realistic under the circumstances or, alternatively, encourage doctors to routinely meet with relatives and fulfil the expectations that have been created.

In addition, nurses also admit that, on occasions, they use doctors as scapegoats in difficult or awkward situations, or if they are unsure of what information to give. This is a method of dispersing some of the responsibility of information giving. Nurses also felt responsible for doctors' actions when they felt that doctors had let families down e.g. if a doctor said that he would see a relative at a certain time and then did not. This was because nurses are always visible on the unit. Figure 14 illustrates what information relatives would like from whom. 


\section{INFORMATION FROM DOCTORS}

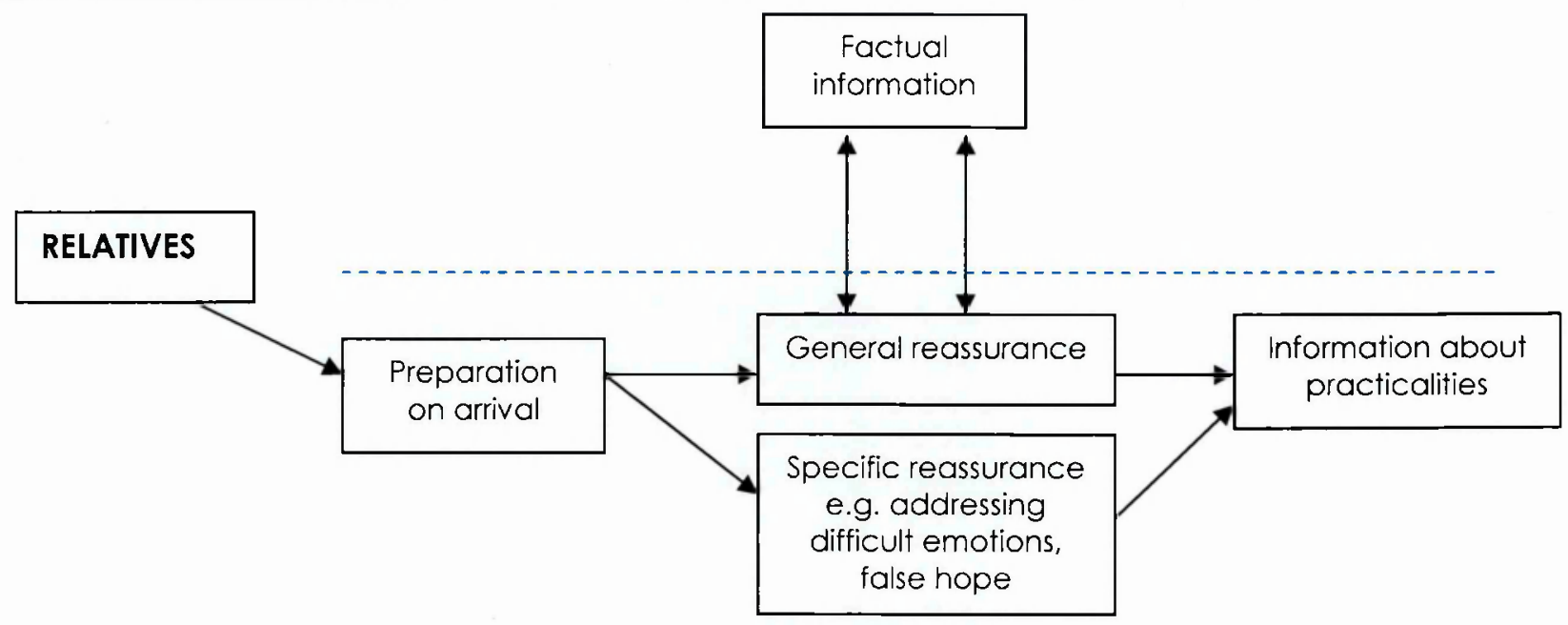

INFORMATION FROM NURSES

Figure 15 - The process of giving information to relatives in critical care

As in Phase I, relatives wanted to receive information about diagnosis and prognosis from doctors. Despite relatives feeling that nurses were competent and capable, the perception was that doctors carry the authority with regarding information giving. Nurses prepared relatives for what to expect on the unit and they accurately perceive how frightening the situation can be for relatives. They are also able to offer reassurance to relatives about the patient because they are constantly present. It is also the nurses who inform relatives about practicalities, although relatives feel that they should not bother nurses with issues such as car parking. $\mathrm{N}$ urses acknowledge that sometimes it is this type of information they forget to tell relatives. Nurses also accurately perceived, as in $\mathrm{P}$ hase $\mathrm{I}$ and in a ccordance $\mathrm{w}$ ith the 1 iterature (Bowman 2000 ), that relatives do not absorb much of the information they are told. This was also i llustrated b y r elatives a dmitting they $\mathrm{c}$ annot $\mathrm{r}$ emember $\mathrm{m}$ uch $\mathrm{a}$ bout the i nitial admission period. 
Contrary to the guidelines laid out in Comprehensive Critical Care Review (Department of Health 2000a), relatives were less concerned about whether they received written information about the workings of the unit. They did want this information, but felt that as they visited the unit every day and stayed as long as possible, receiving the information verbally was adequate. They would have liked to have received written information about the specific condition of their patient. Staff felt that this information was virtually impossible to give because no two cases of the same condition are ever identical. They felt that by attempting to give this information they were exposing themselves to criticism or blame if the patient did not then progress exactly as predicted. This attitude may be associated with the 'blame culture' that is increasing in society. Some nurses also felt that any information that could be put into information leaflets was too general.

Nurse found complex family dynamics difficult to cope with and to minimise confusion tended to give information only to the registered next of kin, they acknowledged how this could cause problems among relatives but felt it was their only option. Relatives, neither in this Phase nor in Phase I raised this as problematic.

Relatives would have liked to have been asked by staff on the unit how they were coping. They felt that although it would not have made a lot of difference to their coping abilities, it would have been nice to have known that the staff cared about them. Relatives acknowledged that the staff offered reassurance about the patient and appreciated this, but felt that this reassurance was not the same thing. Staff felt that they were offering emotional support but also acknowledged the difficulties in maintaining professional boundaries. This is strongly linked to the concept of emotional labour discussed earlier (see Chapter 2, p26). 
Visiting hours were a major issue on the unit whose visiting times were limited. Relatives felt that the time outside of visiting hours was too long to be away from their patient and under these circumstances they would have liked to have had a telephone call for example, to say that their patient had settled for the night. However, it may be more appropriate for the relatives to call the unit. Relatives disliked being asked to leave the unit during visiting hours because they felt that this eroded the limited time they had to spend with their patient. Nurses felt that this could not be avoided on occasion.

An original research objective of this study was to investigate whether there was any evidence to suggest that relatives found their experiences psychologically traumatic. In this Phase relatives exhibited a variety of stress symptoms that did not emerge in the relatives from Phase I. This is an area that should be investigated further to determine the extent to which these symptoms persist after the critical care experience is over.

Relatives felt that waiting facilities were not inadequate when the units were busy, particularly if each patient had large numbers of visitors. They felt that there was nowhere private to go, for example, if they needed to cry. Each unit did have an additional quiet room however relatives had negative cognitions associated with the quiet room attached to each unit. This was the room in which relatives were normally told bad news. Relatives expressed a fear and dislike of this room. It may be advantageous to vary the location of conveying bad news, so that these negative associations do not develop. This way, relatives may still be prepared to use the quiet room as an additional waiting area.

Although some relatives felt that a member of staff whose job was to care for relatives could be useful, others acknowledged that this is impractical and unrealistic. The 
qualities that relatives sought were seeing a familiar face, having someone they could turn to for emotional support if required, someone they could turn to discover the external practicalities of the hospital, such as, car parking and accommodation and someone who could assist with more personal issues, such as financial advice arising from the patient being critically ill. Relatives did not want someone who would replace the contact with the nurse caring for their patient. They felt very strongly that their relationship with the nurse at the bedside was important. Other units already have social workers attached, including one of the units in this study. However, it appeared that the social worker did not routinely talk to patients or relatives and was only involved if the nurses specifically asked for help. Some of the more junior members of staff were unaware that there was a social worker attached to the unit. A regular figure who visited the unit, for the benefit of the relatives, patients and staff would be an invaluable member of the critical care team. Staff confirmed this was successful on other units they had worked on. Staff also discussed how they would not want to reduce contact with relatives as it is a method of building trust. However, they did recognise the benefits of an additional member of staff to help with relatives during the patient's admission when the relatives are not the nurse's priority and when there were long periods of time when relatives were kept waiting. Figure 15 illustrates factors that affect relatives coping and suggests possible additional interactions that could be introduced. The latter are indicated in blue.

Staff identified a gap in training and referred to previous resources that had been available to them, for example one of the units used to receive tailored training for critical care from a staff counsellor. They felt that existing training, regarding difficult situations etc, provided by Hospital Trusts was too generic and not specialised enough for the unique environment of critical care. 


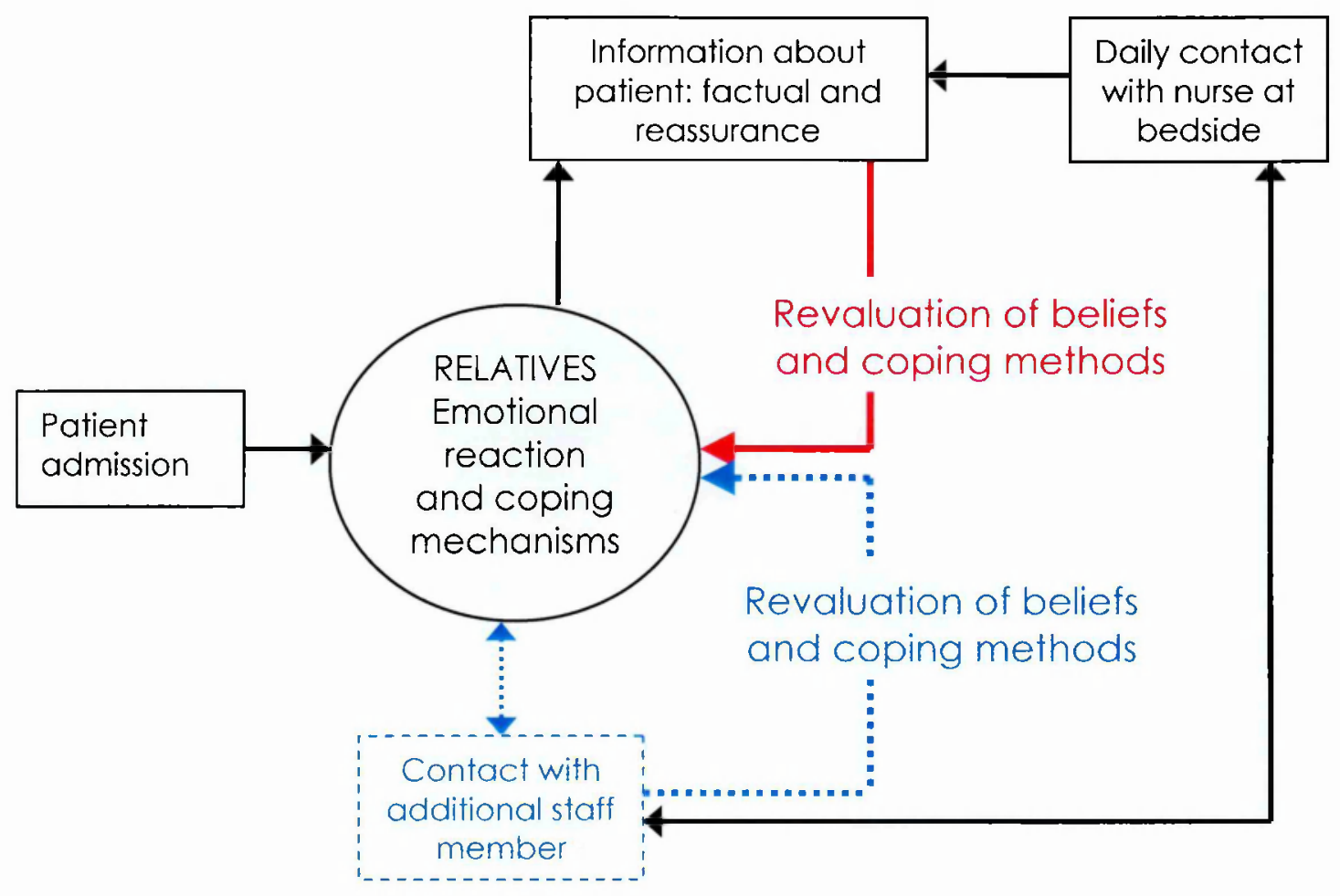

Figure 16 - Factors influencing relatives coping methods while in a critical care environment.

Ultimately, the patient is the critical care nurse's priority and a balance needs to be found with relatives, without losing sight of this. The reasoning behind many nurses' actions is to protect the patient's dignity or the dignity of the other patients on the unit. This may be something that is not made clear enough to relatives. Nurses sometimes find relatives difficult to deal with in today's changing society, they feel that there is a often a lack of respect for what they are trying to achieve and a lack of patience and understanding by relatives of the systems and rules that are in place on the units.

In general, nurses have a difficult task balancing the needs of the patient with the expectation of the relative. Some straightforward protocol changes (e.g. use of different locations to convey bad news, introducing doctors by name) could 
significantly reduce stress levels of relatives. Specialist training for critical care nurses with emphasis on communication with relatives could also enhance interactions with relatives and reduce levels of anxiety. 
Chapter Seven

CROSS STUDY DISCUSSION AND CONCLUSION 


\subsection{Introduction}

This study aimed to investigate relatives' experiences of critical care and staff perceptions of those experiences. To achieve this, the study used a combination of qualitative and quantitative methods of research over three phases. Each phase has been discussed individually in the relevant chapters and this chapter aims to examine the main findings in the context of the original research questions (see chapter 1, p13 for list of research questions).

Each of these studies built upon the findings from the previous phase. Phase I investigated existing gaps in the literature to identify the salient issues surrounding relatives' experiences of critical care from the viewpoint of relatives and staff. The prominent issue appeared to be the process by which relatives received information. Consequently, Phase II explored what written information was available to relatives on a national scale to assess whether there was a consistent nationwide approach to this issue. It also considered the information and guidance made available to staff, in terms of official policies, regarding the care of relatives. The results from Phase II highlighted an inconsistency in the provision of information. It also indicated that Government recommendations were not being met. Therefore, the objective of Phase III was to ascertain whether those recommendations reflect the amount, type and nature of information relatives wanted to receive and how they would like the information to be communicated to them. The views of staff were also sought, to establish how realistic relatives' views were in terms of implementation. 


\subsection{Discussion of Main Findings}

There have been several studies on identifying the needs of relatives (Leske 1986; Molter 1979) with additional follow-up studies on whether these needs are being met (Dyer 1991; Mendonca \& Warren 1998). However, few studies have been carried out on the overall experience of relatives (Zainal \& Scholes 1997) therefore it is important to establish the extent to which nurses can be empathic towards relatives. Although Zainal and Scholes examined relatives' perceived experiences and the perceived contribution made by the critical care nurses, they did not address whether staff were accurately aware of relatives' perceived experiences. In addition, their research was carried out in 1994 within the context of the introduction of primary nursing, which is now commonplace.

A series of themes run through each phase of this research. Generally, relatives are satisfied with their experiences of critical care. For the most part, they are appreciative of the care their patient has received and the professionalism of the staff, which is particularly reflected in the findings from Phase III. Phases I and III found that relatives initially suffer from a shock reaction on discovering that a family member is critically ill and at their first experience of a critical care unit. This is in line with the literature on crisis situations (Caplan 1971), which observes that a crisis arises when one is faced with a problem that is perceived, for a time, as insurmountable and this is followed by a period of confusion and distress. It also corresponds with literature on the effect of the unfamiliar nature of the critical care environment on relatives (Jastremski 2000) and highlights the importance of reassuring relatives about the environment, to reduce fear (Ashworth 1984). In time, relatives become accustomed to the routine of the unit and implement problem focused and emotion focused coping strategies (see chapter 2, p24 for 
a detailed discussion on coping mechanisms). Some go through an anticipatory grief reaction because they fear the death of their family member and it becomes a way for them to prepare for what they perceive as inevitable. Others look for something to hope for, so that they have something positive to focus on; some are philosophical and others try to make themselves useful, for example by becoming involved in patient care. This study illustrated that relatives embark on coping strategies that closely follow Lazarus and Folkman's (Lazarus \& Folkman 1984b) theory of stress and coping, for example, the self deception involved in the maintenance of hope.

Phase I concluded that nurses accurately perceive relatives' experiences whilst in critical care, for instance, they are aware of the emotions experienced by relatives and accurately perceive the threatening nature of the environment, the difficulties relatives face learning about the environment and learning to cope. Nurses also accurately perceive that relatives absorb very little information under the stressful circumstances, which supports findings in previous literature (Bowman 2000). These results were further supported by findings in Phase III.

One research objective was to investigate the extent to which relatives found their experiences to be psychologically traumatic. In Phase I, there was no evidence to suggest that this was the case, however, when interviewing a larger sample in Phase III relatives appeared to suffer from a variety of stress symptoms. Phase I addressed issues around what factors made critical care harder or easier for relatives to deal with and aimed to identify factors that may potentially cause psychological distress. This could support the quantitative work carried out by Jones et al (2004) who found a high incidence of psychological distress in relatives that were persistent at six months post-discharge from 
critical care. It is unclear from these results the extent to which those symptoms are enduring and this would be an interesting area of further research. Qualitative interviews in the immediate critical care environment and at future intervals post discharge could be used to investigate this, together with established quantitative scales, for example, the Hospital Depression and Anxiety Scale (Zigmond \& Snaith 1983) and/or the Impact of Events Scale (Horowitz, Wilner \& Alvarez 1979).

Dissemination of information and communication are topics that have emerged as relevant throughout all three phases. This is an area that seems to have been neglected in research into relatives' needs and experiences in favour of other, perhaps more practical, needs. Fifteen of the original 45 statements that appeared on the Critical Care Family Needs Inventory (CCFNI) (Molter 1979) were concerned with communication issues. Research on communication in critical care units has concentrated on communication between doctors and nurses and/or nurses/doctors and patients. Communication was highlighted in Phase I as a salient issue of concern to relatives and Phase II explored the question of what information relatives had available to them on a national basis. Information leaflets accessible to relatives were examined in the context of Department of Health (DoH) guidelines (Department of Health 2000a). Sixteen percent of the general critical care units that participated in the study were not complying with these guidelines by having no information leaflets and those units that did vary largely in the quality and quantity of information provided. This was a more conservative figure than that published in the Audit Commission report (1999). However, although there is extensive literature to suggest that information reduces anxiety, this does not necessarily need to be written information. Jones et al. (2004) highlight that psychological distress felt by relatives of critically ill patients was not diminished by access to written information. In Phase III, 
relatives appeared less concerned about receiving written information on the areas recommended by the $\mathrm{DoH}$, such as unit practices, facilities available and staff involved in care because they felt they were present on the unit for sufficient time to be given this information verbally. This has implications for the methods by which information is provided. It may prove more cost effective to display general information about the units on notice boards and convey information verbally rather than incurring the expense of printing information leaflets that relatives do not necessarily consider useful. Written information for relatives may not correlate with verbal information that they are given by staff and therefore this can only act as a guide that should be reinforced verbally.

Relatives had specific views regarding the type, amount and nature of information that they wished to receive. They appreciated receiving a mixture of factual information and general reassurance verbally from nurses. All relatives in Phase III reported that they understood what they had been told. In Phase I, it was not clear from the results whether this also was the case. As for written information, leaflets in Phase II generally had a score greater than the FOG readability index score of 20 or under, recommended for information of this type. It is therefore possible that some of the written information provided to relatives may not have been fully understood.

It was perceived that doctors did not routinely talk to relatives and consequently relatives felt that their contact with doctors is inconsistent. Many relatives also stated that doctors frequently did not introduce themselves. In addition, nurses perceived that relatives are more satisfied once they have spoken to a doctor and, therefore, would encourage a system whereby doctors spoke to relatives routinely. Critical care is a particularly nurse led environment and it would be useful to investigate why relatives feel the need to have 
regular contact with doctors, when in fact, the nurses who care for their patients are possibly in a better position to inform relatives of their patient's progress. If, indeed, this is a reflection of media portrayal of hospital settings in television dramas, as indicated in Phase I, then this issue needs to be addressed. Nurses in Phase I perceived that the media gives relatives unrealistic expectations. It may be more cost effective and time efficient if, on arrival at the unit, it was explained to relatives that they would not be seeing a doctor unless, for example, there was a significant change in the patient's condition or treatment and that the nurses were likely to be more informed about the patients care. Many nurses currently feel that that they are under pressure to encourage doctors to see relatives, despite time constraints, and as a consequence feel guilty when doctors say they will do something and then do not manage to. Promoting the competence of the nurses to relatives, encouraging doctors to introduce themselves and clarifying doctors contact procedures with relatives may alleviate anxiety levels.

In addition to being given information directly relating to their patient, relatives would like to have been asked whether they were coping, although it made no material difference to their position, to show that the hospital personnel cared about their welfare in addition to that of the patient. This was not a need listed on the original CCFNI and therefore may have been neglected in the plethora of replication work since. Whether it is the nurse's job to offer emotional support to relatives is a grey area in nursing literature, however, it is known that social support aids successful coping (Lazarus \& Folkman 1984a) and lack of positive social support induces negative psychological states, such as depression or anxiety. Both the buffering model and the direct effect model of coping identify social support as an influential factor. The former cites that social support eases the impact of a stressor when it occurs whereas the latter claims constant social support is 
beneficial for psychological health regardless of whether a stressor is present (Pennix et al. 1998). For some relatives nurses may be the only means of support and therefore this would illustrate the existence of the buffering model. This may be an area in which an additional member of staff on the unit could make a useful contribution by dealing with the practicalities that relatives feel are not part of the nurses remit (e.g. parking permits and financial concerns). Nurses in Phase III indicated that an additional member of staff worked successfully on other units and relatives indicated that it would have been useful to see a regular, familiar face. This position could perhaps be filled by a Health Care Assistant who had received additional training on specific advice and welfare areas. This concept has emerged sporadically in nursing literature since the 1970s (Wallace 1971) without any empirical investigation to confirm whether it is a viable option and again, this may be a useful area of future research.

This research also supports previous studies, which illustrated the importance for relatives to feel hope (Coulter 1989; Patel 1996; Zainal \& Scholes 1997). Hope is an important element for successful coping strategies and it ultimately offers a measure of protection against the state of despair (Miller 1989). When communicating with relatives, doctors and nurses should be directly aware of making statements that indicate hope, or lack of it, as this may influence the ability, or lack of ability, of relatives to cope. In this research, relatives in both Phases I and III commented on the despair of constantly receiving negative information. Giving hope is a means of support and again illustrates the existence of the buffering model of social support in aiding successful coping strategies. In addition, the way in which that information is delivered can also impact on levels of hope. The importance of this was conveyed by a relative in Phase I who acknowledged that she was devastated following a conversation with a doctor, only to 
realise later that the information she was told was not new, but the way in which the information had been communicated "destroyed" her hope.

Despite investigating two very different critical care settings (general and neurological) differences in the experience of relatives were not overly apparent. This is contrary to findings by Mathis (1984) who carried out a quantitative study on the needs of relatives of critically patients with and without acute brain injury. However, Mathis acknowledged the design of the experiment may not have been appropriate for detecting differences in her results. Differences between relatives of patients with and without brain injury were highlighted in Phase I, but these may have appeared to be a result of the time lapse between the critical care experience and the interview, rather than the critical care experience itself. Differences in Phase III seemed to be due to variances in visiting hours between the units rather than the nature of the illness or injury. There also appeared to be little difference between the experiences of relatives regardless of whether their patient's admissions were planned or unplanned, despite the assumption that relatives of planned admissions have more time to prepare psychologically for the event. This supports work by Twibell (1998) who found that suddenness of admission was not related to coping effectiveness and previous research into the beneficial effects of pre-operative tours show that, despite anecdotal evidence to the contrary, preoperative tours have no statistically significant benefit in reducing anxiety of relatives (Lynn-McHale et al. 1997). Instead, the results suggest that it is the extent of relatives' previous experience or medical knowledge that eases the stressful nature of critical care. Although this may be regarded as commonsense, it is something that is not highlighted in the literature and may be of importance to establish when a relative arrives on the unit. 
Nurses in Phases I and III identified a gap in their training and this was also further illustrated in Phase II by the lack of official policies units had available for staff, to offer guidance on how to care for relatives. Such guidance might be particularly important to less experienced staff who are new to critical care. As critical care is unlike any other unit in the hospital, many nurses felt that training provided by Hospital Trusts was not sufficiently specific. Most nurses considered that, as emotions are more extreme in this highly tense environment, training in dealing with aggressive relatives and difficult situations specific to critical care would be useful. Training in dealing generally with relatives, possibly involving aspects of communication training and awareness of what relatives are experiencing would also be appreciated.

In addition to relatives' concerns about communication processes, there were some practical issues that need to be addressed, such as the reason why some units have open visiting hours and others do not. Relatives whose patient had been transferred from another critical care unit with fewer visiting restrictions struggled to understand the reasons for the differences in unit practices. There is an obvious necessity for procedures, such as ward rounds and physiotherapy, and nurses acknowledge that they can find the constant presence of some relatives draining. However, if there are fewer visiting restrictions, in addition to enhancing national consistency, relatives may be more relaxed and, in turn, may be less demanding of nurses.

Relatives in both Phases I and III generally thought that waiting facilities were inadequate for the size of the units and the number of visitors, particularly if a patient on the unit had a very large family or many friends. In Molter's (1979) study the need to have a waiting room in close proximity to the patient was ranked as the third most important need (out of 
45) for most relatives, however no questions were asked about the adequacy of those facilities. To have comfortable furnishings in the waiting room was ranked 16. Relatives in this study were less concerned with the furniture and more with the size of the space available. They would have appreciated more small rooms, giving a degree of privacy, rather than one large room. This was a concept raised by staff in Phase I as a suggested improvement to relatives' experiences. In practice, the possibilities of alterations to existing units are limited, but it is an important consideration when designing and planning new critical care units. Relatives also developed negative connotations in regard to quiet rooms attached to each unit because these were the rooms where bad news was always conveyed. Varying this location may also contribute to lower stress levels of relatives. Nurses in Phase I and III also raised the issues that arose from one patient having a large number of visitors. For nurses it was the quantity of visitors that proved more problematic than the occasional "difficult" visitor. In addition to placing restrictions on the number of visitors allowed by the bedside at any one time, it may also be prudent to place restrictions on the number of visitors per patient allowed in the waiting room.

Relatives from Phase I placed importance on being involved in patient care. However, they felt that they were not encouraged to participate in this, for example, many were not informed whether or not it was an option. Nurses in Phase III also raised this issue and believed that involving relatives in patient care would be a positive step. This was not an issue that arose among the relatives in Phase III and is another potential area of research. The question of patient consent of relatives' involvement would need to be addressed although, where appropriate, nurses generally would like to see relatives becoming more involved in the basic care of patients. Making relatives feel useful is beneficial for their coping mechanisms by employing problem focused methods, similar to those proposed in 
Lazarus and Folkman's (1984) theory of stress and coping, to help relatives learn new skills or procedures, and the resulting additional free time may enable nurses to perform more complex tasks. Previous research shows that having relatives involved in patient care can aid patient recovery (Dyer 1991). This would only be suitable for longer stay patients as, initially, it may be time consuming for nurses to teach tasks to relatives. The predominant implications for practice would be that relatives will need to be informed that there would potentially be an opportunity for them to be involved in patient care and then they would need to be regularly informed of their patient's progress. They would also need to be explicitly asked if this was an area in which they would like to participate and continuous support from staff would be required.

This research adds a qualitative perspective to the existing quantitative literature. It updates previous qualitative studies that have become dated in the fast changing NHS and it also explores the national picture of provision of information to relatives, something that has not been attempted previously. The relatively small sample size in Phases I and III limits the generalisability of the qualitative results, however, these results are stable across the studies. In addition, the population studied is very specific and consequently there is a need for replication and expansion of the sample population to include different critical care units, different wards and geographical variations, together with input from other staff members working in critical care units.

The findings from this study relating to the factors that affect how relatives cope and how staff are able to care for relatives integrate three theories: Cohen and Wills' theory of social support (1985), Lazarus and Folkman's theory of stress and coping (1984b) and Hochschild's theory of emotional labour (1983b). The first two are psychological theories 
that aim to address the factors that influence how individuals mentally cope with stressful situations. The latter theory is sociological and approaches the same situation from a more social, rather than individual, perspective. These different theoretical positions have been integrated with the aim of gaining a more holistic view of the interactions between nurses and relatives in their experiences in critical care.

The nature of the communication processes between nurses and relatives provides relatives with information which, if adequate, can enable them to implement the problemor emotion focused coping strategies discussed by Lazarus and Folkman (1984). Consequently they will try and use these strategies to deal with the situation in which they find themselves and reduce their stress and anxiety levels. An example of a problem focused strategy was becoming involved in basic patient care. An example of an emotion focused strategy was to think about the positive aspects of the situation, such as the patient was in the best possible place.

Relatives expressed a wish for staff to enquire whether they were coping and would like this to be explicitly addressed. Offering this explicit social support may aid the coping strategies that relatives use and as such illustrate how the buffering model of social support (Cohen \& Wills 1983) exists within this environment. The buffering model states that social support eases the impact of a stressor at the time it occurs, e.g. a social network can reduce anxiety levels.

Whilst caring for relatives nurses implicitly employ methods explicitly outlined in the theory of emotional labour (Smith, 1992) to manage their own emotional needs within their professional boundaries. Dealing with different needs of relatives may generate different types of emotional labour for nurses in order to fulfill the relatives' expectations 
of support. Previous studies have found that nurses felt better able to care when they felt cared for themselves (Smith 1992). This may be done through staff support mechanisms. Making explicit the emotional demands of nursing may go some way to reduce these misconceptions.

\subsection{Conclusion}

This study shows that, in general, relatives are content with their critical care experiences. This is a positive finding that should be publicised to encourage a workforce that appear to be feeling increasingly undervalued. Relatives wanted to be kept informed of all stages of their patient's condition and received a mixture of factual information and reassurance which they found helpful. It is important that these aspects are not lost amid further improvements and developments.

This research indicates four main areas that have implications for practice. Firstly, clear guidelines need to be established for both nurses and relatives as to how often relatives should expect contact with doctors. This would aim to allay expectations, largely created by the media, about the desirability of the presence of doctors and would reduce feelings of responsibility for the actions of doctors experienced by nurses. In addition, cost effective methods of relaying general information to relatives should be considered, for example, notice boards in waiting areas as opposed to information leaflets.

Secondly, there may be a need for the employment of an additional member of staff, for example, a Health Care Assistant, who had received extra critical care specific training, to act as an information point for relatives. This could be helpful by dealing with practicalities, such as car parking, financial concerns or providing contact details for 
external support organisations. This person could also be the regular familiar face that many relatives desire and may go some way to addressing the overlap of skills that critical care nurses encounter in the tasks they perform.

Thirdly, the gap in training that nurses highlighted needs to be addressed. Further training in communication, conveying difficult information and dealing with the responsibilities of relatives' complex emotional processes could be offered, ensuring that this training is relevant to the critical care setting.

Finally, encouraging relatives to become involved in basic patient care, where appropriate, could have beneficial effects for patients, relatives and nurses, particularly in longer stay patients. Although it may be initially time consuming for nurses to have to show relatives how to carry out tasks correctly, in the longer term, it could aid relatives' coping abilities by making them feel that they have a purpose. It could also free up nurses time to perform more complex tasks and provide comfort for the patient by fostering awareness that they are being cared for by a family member.

To build on this research, recommendations for future investigation include examining in more detail the relationships within a relative-nurse-doctor triad, using an emotional labour framework. In addition, exploring the longer term impact of critical care on relatives, post patient discharge, is an area that may well contribute to improving their general psychological well being. 


\section{REFERENCES}

American Psychiatric Association 1994, Diagnostic and statistical manual of mental disorders, 4th edn, American Psychiatric Association, Washington DC.

Ashworth, P. M. 1984, "S taff-patient communication in coronary care units", Journal of Advanced Nursing, vol. 9, pp. 35-42.

Audit Commission 1993, What seems to be the matter: communication between hospital and patients., HMSO, London, National Health Service Report No 12.

Audit Commission 1999, Critical to success: the place of efficient and effective critical care services within the acute hospital, Holbrooks Printers, Portsmouth.

Authority of the House of Lords. Mental Capacity Bill. HL Bill 13. 2003. Ref Type: Bill/Resolution

Bennun, I. 2001, "Intensive care unit syndrome: A consideration of psychological interventions", The British Journal of Medical Psychology, vol. 74, pp. 369-377.

Booth, M. G., Doherty, P., Fairgrieve, R., \& Kinsella, J. 2004, "Relatives' knowledge of decision making in intensive care", Journal of Medical Ethics, vol. 30, pp. 459-462.

Bowman, K. W. 2000, "Communication, Negotiation, and Mediation: Dealing with Conflict in Endof-Life Decisions.", Journal of Palliative Care, vol. 16, no. Supplement, p. S17-S23.

Bratt, M. M., Broome, M., Kelber, S., \& Lostocco, L. 2000, "Influence of stress and nursing leadership on $\mathrm{j}$ ob s atisfaction of $\mathrm{p}$ ediatric intensive c are unit n urses", A merican Journal of Critical Care, vol. 9, no. 5, pp. 307-317.

Breakwell, G. M., Hammond, S., \& Fife-Schaw, C. 1995, Research Methods in Psychology SAGE Publications Ltd, London.

Breu, C. \& D racup, K. 1978 , "H elping the spouses of critically ill patients.", American Journal of Nursing, vol. 78 , no. 1, pp. 50-53.

Britten, N. 1999, "Qualitative interviews in health care research," in Qualitative interviews in health care research, 2nd edn, C. Pope \& N. Mays, eds., BMJ Books, London, pp. 11-19.

Burns, N. \& Grove, S. K. 2001a, "Introduction to qualitative research," in The practice of nursing research: Conduct, critique and utilization, 4th edn, W. B. Saunders Company, Philadelphia, pp. 6182.

Burns, N. \& Grove, S. K. 2001b, "Ethics and research," in The practice of nursing research: Conduct, critique and utilization, 4th edn, W.B.Saunders Company, Philadelphia, pp. 191-222.

Butler, L. D., Koopman, C., Classen, C., \& Spiegel, D. 1999, "Traumatic stress, life events and emotional support in women with metastatic breast cancer: Cancer-related traumatic stress symptoms associated with past and current stressors.", Health Psychology, vol. 18, no. 6, pp. 555-560.

Caplan, G. 1971, "A community approach to preventive psychiatry," in An Approach to Community Mental Health., Tavistock Publications, Great Britain, pp. 1-31.

Carlisle, D. 2003, "Making patient leaflets work", Nursing Times, vol. 99, no. 6, p. 40.

Castledine, G. 2001, "Is nursing care losing its holistic focus?", British Journal of Nursing, vol. 10, no. 16, p. 1091.

Centre for Health Information Quality. 2000, www.hfht.org/chiq.guidelines.htm Ref Type: Internet Communication

Cohen, S. \& Wills, T. A. 1985, "Stress, social support and the buffering hypothesis.", Psychological 
Bulletin, vol. 98, no. 2, pp. 310-357.

Cornock, M. A. 1998, "Stress and the intensive care patient: perceptions of patients and nurses", Journal of Advanced Nursing, vol. 27, pp. 518-527.

Coulter, M. 1989, "The needs of family members of patients in intensive care units", Intensive Care Nursing, vol. 5, no. 4, p. 10.

Cox, T. 1988, "Psychobiological factors in Stress and Health," in Handbook of Life Stress, Cognition and Health, S. Fisher \& J. Reason, eds., John Wiley \& Sons, Chichester, pp. 603-625.

Crawford, P., Brown, B., \& Nolan, P. 1998, Communicating Care: The Language of Nursing Stanley Thornes Publishers Ltd, Cheltenham.

Creswell, J. W. 1994, Research Design: Qualitative and quantitative approaches Sage Publications, Inc., Thousand Oak.

de Raeve, L. 1994, "Ethical issues in palliative care research", Palliative Medicine, vol. 8, pp. 298-305

Denzin, N. K. \& Lincoln, Y. S. 2000, Handbook of qualitative research, 2nd edn, Sage Publication Inc, Thousand Oaks.

Department of Health 2000a, Comprehensive Critical Care: A review of adult critical care services. The Stationary Office, London.

Department of Health 2000b, The NHS Plan: A plan for investment, a plan for reform., The Stationary Office, London.

Department of Health 2001, The nursing contribution to the provision of comprehensive critical care for adults: A strategic programme of action, Department of Health, London.

Department of Health 2003, Strengthening Accountability: Involving patients and the public. Practice Guidance. Section 11 of the Health and Social Care Act 2001. Crown Copyright, London.

Duman, M. \& Farrell, C. 2000, The POPPi Guide: Practicalities of producing patient information., 1st edn, King's Fund Publishing, London.

Dyer, I. D. 1991, "Meeting the needs of visitors - a practical approach", Intensive Care Nursing, vol. 7, pp. 135-147.

Dyer, I. D. 1997, "Research into visitor needs using Molter's tool - time to move on.", Nursing in critical care, vol. 2, no. 6, pp. 285-290.

Ehlers, A. \& C lark, D. M. 2000, "A cognitive model of posttraumatic stress disorder", B ehaviour Research and Therapy, vol. 38, pp. 319-345.

Fielding, N. G. 1982, "Observational research on the national front," in Social Research Ethics, M. Bulmer, ed., Macmillan, London.

Fielding, R. G. \& Llewelyn, S. P. 1987, "Communication training in nurses may damage your health and enthusiasm: some warnings", Journal of Advanced Nursing, vol. 12, pp. 281-290.

Folkman, S. 1997, "Positive psychological states and coping with severe stress", Social Science and Medicine, vol. 45, no. 8, pp. 1207-1221.

Folkman, S. \& Lazarus, R. S. 1988, "Coping as a mediator of emotion", Journal of Personality and Social Psychology, vol. 54, no. 3, pp. 466-475.

Forrester, D. A., Murphy, J. F., Price, D. M., \& Monaghan, J. F. 1990, "Critical care family needs: Nurse-family member confederate pairs", Heart and Lung, vol. 19, no. 6, pp. 655-661.

Fulton, G., Madden, C., \& Minichiello, V. 1996, "The social construction of anticipatiry grief.", Social Science and Medicine, vol. 43, no. 9, pp. 1349- 1358. 
Gilhooly, K., Gill, R., Green, C., Hammersley, M., Henwood, K., King, E., Pidgeon, N., Potter, J., Rachel, J., Smith, J., Toren, C., \& Woolgar, S. 2003, Handbook of Qualitative Research Methods for Psychology and the Social Sciences The British Psychological Society, Leicester.

Gillham, B. 2000, The research interview Continuum, London.

Glaser, B. G. \& Strauss, A. L. 1967, The Discovery of Grounded Theory: strategies for qualitative research Aldine de Gruyter, New York.

Goffman, E. 1959, The presentation of self in everyday life Doubleday Anchor, New York.

Goodfellow, A., Varnam, R., Rees, D., \& Shelly, M. P. 1997, "Staff stress on the intensive care unit: a comparison of doctors and nurses", Anaesthesia, vol. 52, no. 11, pp. 1037-1041.

Granberg, A., Engberg, I. B., \& Lundberg, D. 1996, "intensive care syndrome: a literature review", Intensive and Critical Care Nursing, vol. 12, no. 173, p. 182.

Green, A. 1996, "An exploratory study of patients' memory recall of their stay in an adult intensive therapy unit", Intensive and Critical Care Nursing, vol. 12, pp. 131-137.

Gregurek, R. 2001, "Increase of frequency of post-traumatic stress disorder in disabled war veterans during prolonged stay in a rehabilitation hospital", Croatian Medical Journal, vol. 42, pp. 161-164.

Habermas, J. 1987, The philosophical discourse of modernity: twelve lectures Polity, Cambridge.

Hamberg, K., Johansson, E., Lindgren, G., \& Westman, G. 2005, "Scientific rigour in qualitative research: Examples from a study on women's health in family practice.", Family Practice, vol. 11, no. 2, pp. 176-181.

Hammond, F. 1995, "Involving families in care within the intensive care environment: a descriptive survey", Intensive and Critical Care Nursing, vol. 1, pp. 256-264.

Henneman, E. A., Mckenzie, J. B., \& Dewa, C. S. 1992, "An evaluation of interventions for meeting the information needs of families of critically ill patients", American Journal of Critical Care, vol. 1, no. 3, pp. 85-93.

Heyland, D. K., Rocker, G. M., Dodek, P. M., Kutsogiannis, D. J., Konopad, E., Cook, D. J., Peters, S., Tranmer, J. E., \& O'Callaghan, C. J. 2002, "Family satisfaction with care in the intensive care unit: Results of a multiple center study", Critical Care Medicine, vol. 30, no. 7, pp. 1413-1418.

Hinds, C. J. \& W atson, D. 1996, "Planning, Organisation a nd M anagement," in Intensive Care: A concise textbook, 2nd edn, WB Saunders Company Limited, London, pp. 1-18.

Hickey, M. 1990, "What are the needs of families of critically ill patients? A review of literature since 1976.", Heart and Lung, vol. 19, no. 4, pp. 401-415.

Hickey, M. \& Lewandowski, L. 1988, "Critical care nurses' role with families: A descriptive study", Heart and Lung, vol. 17, no. 6 part 1, pp. 670-676.

Hochschild, A. 1983a, "Between the toe and the heei: jobs and emotional labor," in The Managed Heart: commercialization of human feeling, University of California Press, Berkeley, pp. 137-161.

Hochschild, A. 1983b, The Managed Heart: commercialization of human feeling University of California Press, Berkeley.

Holloway, I. 1997, Basic concepts for qualitative research. Blackwell Science, Oxford.

Hoover, M. J. 1979, "Intensive care for relatives", Hospitals, vol. July 16 1979, pp. 219-222.

Horowitz, M., Wilner, N., \& Alvarez, W. 1979, "Impact of Events Scale: a measure of subjective stress", Psychosomatic Medicine, vol. 14, pp. 209-218.

Hudson, G. R. 1993, "Empathy and technology in the coronary care unit", Intensive and Critical Care 
Nursing, vol. 9, pp. 55-61.

Hughes, F., Robbins, I., \& Bryan, K. 2004, "An analysis of information available to relatives in intensive care." Intensive and Critical Care Nursing, vol 20 no. 4. pp200-205.

Hupcey, J. E. 1999, "Looking out for the patient and ourselves - the process of family intergration into the ICU.", Journal of Clinical Nursing, vol. 8, pp. 253-262.

Intensive Care Society 2003, Evolution of intensive care in the UK, Intensive Care Society, London. Intensive Care Society 1990, Intensive care service in the UK Intensive Care Society, London.

Janis, I. L. 1982, Groupthink: psychological studies of policy decisions and fiascoes Houghton Mifflin, Boston.

Jastremski, C. A. 2000, "ICU bedside environment. A nursing perspective.", Critical Care Clinics, vol. 16, no. 4, pp. $723-734$.

Jennings, T. 2001, "Reading between the lines", NHS Magazine, vol. November, pp. 20-21.

Johnson, M. \& Webb, C. 1995, "Rediscovering unpopular patients: the concept of social judgement." Journal of Advanced Nursing, vol. 21, no. 3, pp. 466-475.

Jones, C., Griffiths, R. D., \& Humphris, G. M. 1998, "Psychological morbidity following critical illness - The rationale for care after intensive care.", Clinical Intensive Care, vol. 9, pp. 199-205.

Jones, C., Skirrow, P., Griffiths, R. D., Humphris, G. M., Ingleby, S., Eddleston, J., Waldmann, C., \& Gager, M. 2004, "Post traumatic stress disorder-related symptoms in relatives of patients following intensive care", Intensive Care Medicine, vol. 30, pp. 456-460.

Kelman, H. C. 1972, "The rights of the subject in social research: an analysis in terms of relatives power and legitimacy", American Psychologist, vol. 27, pp. 989-1016.

Kenny, T., Wilson, R. G., \& Purves, I. N. e. a. 1998, "A PIL for every ill? Patient information leaflets (PILs): a review of past, present and future use.", Family Practice, vol. 15, no. 5, pp. 471-479.

King, S. L. \& Gregor, F. M. 1985, "Stress and Coping in Families of the Critically Ill", Critical Care Nurse, vol. 5, no. 4, pp. 48-51.

Kuhn, T. S. 1970 , The structure of scientifc revolutions. $2^{\text {nd }}$ Edition. Chicago University Press, Chicago.

Lawler, J. 1991, "Body care and learning to do for others," in Behind the Screens: Nurses, somology and the problem of the body, Churchill Livingstone, Melbourne, pp. 117-133.

Lazarus, R. S. 1966, Psychological stress and the coping process McGraw Hill, New York.

Lazarus, R. S. \& Folkman, S. 1984a, "The coping process: An alternative to traditional formulations," in Stress, appraisal and coping., Springer Publishing Company, New York, pp. 141-180.

Lazarus, R. S. \& Folkman, S. 1984b, "The concept of coping," in Stress, Appraisal and Coping, Springer Publishing Company, New York, pp. 117-140.

Lee, R. 1993, Doing research on sensitive topics, Sage Publications Ltd, London.

Leske, J. S. 1986, "Needs of relatives of critically ill patients: A follow-up", Heart and Lung, vol. 15, no. 2, pp. 189-193.

Lincoln, Y. S. \& Guba, E. G. 1985, Naturalistic Inquiry Sage Publications, Inc., Newbury Park.

Llenore, E. \& Ogle, K. R. 1999, "Nurse-patient communication in the intensive care unit: a review of the literature", Australian Critical Care, vol. 12, no. 4, pp. 142-145.

Lynch, J., Appelboam, R., \& McQuillan, P. J. 2003, "Survey of abuse and violence by patients and 
relatives towards intensive care staff", Anaesthesia, vol. 58, pp. 874-910.

Lynn-McHale, D. J., Corsetti, A., Brady-Avis, E., Shaffer, R., McGrory, J., \& Rothenberger, C. 1997, "Preoperative ICU tours: Are they helpful?", American Journal of Critical Care, vol. 6, no. 2, pp. 106115.

Maguire, P. 1992, "Improving the recognition and treatment of affective disorders in cancer patients," in Recent Advances in Clinical Psychiatry, K. Granville-Grossman, ed., Churchill Livingstone, Edinburgh, pp. 15-30.

Mathis, M. 1984, "Personal N eeds of Family Members of Critically Ill Patients W ith and W ithout Acute Brain Injury", Journal of Neurosurgical Nursing, vol. 16, no. 1, pp. 36-44.

Mays, N. \& Pope, C. 2000, "Quality in health research," in Qualitative research in health care, 2nd edn, C. Pope \& N. Mays, eds., BMJ Publishing Group, London, pp. 89-102.

Mays, N. \& Pope, C. 1995, "Qualitative research: Rigour and qualitative research", The British Medical Journal, vol. 311, no. 6997, pp. 109-112.

Mendonca, D. \& Warren, N. A. 1998, "Perceived and unmet needs of critical care family members", Critical Care Nursing Quarterly, vol. 21, no. 1, pp. 58-67.

Miles, M. B. \& Huberman, A. M. 1994, Qualitative Data Analysis, 2nd edn, Sage Publications Inc., Thousand Oaks, California.

Miller, J. F. 1989, "Hope-inspiring strategies of the critically ill", Applied Nursing Research, vol. 2, no. 1, pp. 23-29.

Miller, J. F. 1991, "Developing and maintaining hope in families of the critically ill", AACN Clinical Issues in Critical Care Nursing, vol. 2, no. 2, pp. 307-315.

Mirr, M. P. 1991, "Factors affecting decisions made by family members of patients with severe head injury", Heart and Lung, vol. 20, no. 5, pp. 228-235.

Molter, N. C. 1979, "The needs of relatives of critically ill patients: A descriptive study.", Heart and Lung, vol. 8, no. 2, pp. 332-339.

McGaughey, J. \& Harrisson, S. 1994a, "Understanding the preoperative information needs of patients and their relatives in intensive care units", Intensive and Critical Care Nursing, vol. 10, pp. 186-194

McGaughey, J. \& Harrisson, S. 1994b, "Developing an information booklet to meet the needs of intensive care patients and relatives", Intensive and Critical Care Nursing, vol. 10, pp. 271-277.

McMillan, T. M. 2001, "Errors in diagnosing post-traumatic stress disorder after traumatic brain injury", Brain Injury, vol. 15, no. 1, pp. 39-46.

Nievaard, A. C. 1987, "Communication climate and patient care: causes and effects of nurses' attitudes to patients", Social Science and Medicine, vol. 24, no. 9, pp. 777-784.

Oh, T. E. 1997, "Design and orgnisation of intensive care units," in Intensive care manual, 4th edn, T.E.Oh, ed., Butterworth-Heinemann, Oxford, pp. 3-10.

O'Neill Norris, L. \& Grove, S. K. 1986, "Investigation of selected psychosocial needs of family members of critically ill adult patients.", Heart and Lung, vol. 15, no. 2, pp. 194-199.

Patel, C. 1996, "Hope inspiring strategies of spouses of critically ill adults", Journal of Holistic Nursing, vol. 14, no. 1, pp. 44-65.

Patton, M. Q. 2002, "Qualitative Interviewing," in Qualitative Research and Evaluation Methods, 3rd edn, Sage Publications Inc., Thousand Oaks, pp. 339-427.

Paul, F. 2001, "Patient information: involving the user groups", Professional Nurse, vol. 16, no. 10, 
pp. 1405-1408.

Peebles-Kleiger, M. J. 2000, "Pediatric and neonatal intensive care hospitalization as traumatic stressor: Implications for intervention.", Bulletin of the Menninger Clinic, vol. 64, no. 2, pp. 257-280.

Peel, N. 2003, "The role of the critical care nurse in the delivery of bad news", British Journal of Nursing, vol. 12, no. 16, pp. 966-971.

Pennix, B. W. J. H., van Tilberg, T., Boeke, J., Deeg, D. J. H., Kriegsman, D. M. W., \& van Eijk, J. Th. M. 1998, "Effects of Social Support and Personal Coping Resources on Depressive Symptoms: Different for Various Chronic Diseases?", Health Psychology, vol. 17, no. 6, pp. 551-558.

Pochard, F., Azoulay, E., Chevret, S., Lemaire, F., Hubert, P., Canoui, P., Grassin, M., Zittoun, R., le Gall, J.-R., Dhainaut, J. F., \& Schlemmer, B. 2001, "Symptoms of anxiety and depression in family members of intensive care unit patients: Ethical hypothesis regarding decision-making capacity.", Critical Care Medicine, vol. 29, no. 10, pp. 1893-1897.

Pope, C. \& Mays, N. 1995, "Qualitative research: Reaching the parts other methods cannot reach: an introduction to qualitative methods in health and health services research.", The British Medical Journal, vol. 311, no. 6996, pp. 42-45.

Porter, R. 1997, The greatest benefit to mankind: A medical history of humanity from antiquity to the present Harper Collins Publishers, London.

Potter, P. A. \& Perry, A. G. 2001, Fundamentals of Nursing, 5th edn, Mosby, St. Louis.

Price, D. M., Forrester, D. A., Murphy, P. A., \& Monaghan, J. F. 1991, "Critical care family needs in an urban teaching medical center.", Heart and Lung, vol. 20, no. 2, pp. 183-188.

Pryzby, B. J. 2005, "Effects of nurse caring behaviours on family stress reposnses in critical care.", Intensive and Critical Care Nursing, vol 21, no. 1, pp 16-23.

Qualitative Solutions and Research. QSR NUD*IST: Qualitative Data Analysis for Research $\begin{array}{llllll}\text { Professionals. } & \text { [6]. 2003. Victoria, Australia, } & \text { QSR Ltd. }\end{array}$ Ref Type: Computer Program

Quinn, S., Redmond, K., \& Begley, C. 1996, "The needs of relatives visiting adult critical care units as percieved by relatives and nurses. Part 2.", Intensive and Critical Care Nursing, vol. 12, pp. 239-245.

Rachman, S. 2001, "Emotional processing, with special reference to post-traumatic stress disorder", International Review of Psychiatry, vol. 13, pp. 164-171.

Reber, A. S. 1995, Dictionary of Psychology, 2nd edn, Penguin Books Ltd, London.

Reiser, S. L. 1993, "The science of Diagnosis: Diagnostic Technology," in Companion Encyclopaedia of the History of Medicine, Vol 2 edn, W. F. Bynum \& R. Porter, eds., Routledge, London.

Ridley, S., Chrispin, H., Scotton, H., Rogers, J., \& Lloyd, D. 1996, "Changes in quality of life after intensive care: comparison with normal data", Anaesthesia, vol. 52, pp. 195-202.

Rosenzweig, M. R., Leiman, A. L., \& Breedlove, S. M. 1996, Biological Psychology Sinauer Associates, Massachusetts.

Royal National Institute for the Blind. 1998, See it right: Clear print guidelines. London, RNIB. Ref Type: Pamphlet

Ruesch, J. \& Bateson, G. 1968, Communication: The social matrix of psychiatry., 2nd edn, WW Norton, New York.

Sarafino, E. P. 1998, Health Psychology: biopsychsocial interactions, 3rd edn, Wiley, New York.

Sarantakos, S. 1998, Social Research, 2nd edn, Palgrave, New York. 
Schwandt, T. A. 1997, Qualitative inquiry. A dictionary of terms. Sage Publications, London.

Sieber, J. E. \& Stanley, B. 1988, "Ethical and professional dimensions of socially sensitive research", American Psychologist, vol. 43, pp. 49-55.

Skirrow, P., Jones, C., Griffiths, R. D., \& Kaney, S. 2001, "Intensive care - easing the trauma", The Psychologist, vol. 14, no. 12, pp. 640-642.

Smith, P. 1992, The Emotional Labour of Nursing: How nurses care, Macmillan Education Ltd, London.

Stanislavski, C. 1965, An Actor Prepares Theatre Arts Books, New York.

Strauss, A. L. \& Corbin, J. 1998, Basics of qualitative research: techniques and procedures for developing grounded theory, 2nd edn, Sage Publication, Inc., Thousand Oak.

Turnbull, G. J. 1998, "A review of post-traumatic stress disorder. Part II: Treatment", Injury, vol. 29, no. 3 , pp. 169-175.

Turnock, C. 1991, "Communicating with patients in ICU", Nursing Standard, vol. 5, no. 15/16, pp. 3840.

Twibell, R. S. 1998, "Family Coping during Critical Illness", Dimensions of Critical Care Nursing, vol. 17, no. 2, pp. 100-112.

Wallace, P. 1971, "Relatives should be told about intensive care - but how much and by whom?", The Canadian Nurse, vol. June 1971, pp. 33-34.

Wild, C. \& Narath, M. 2004, "Evaluating and planning ICUs: methods and approaches to differentiate between need and demand.", Health Policy.

Wiles, R. 1998, "Patients' perceptions of their heart attack and recovery: the influence of epidemiological "evidence" and personal experience.", Social Science and Medicine, vol. 46, no. 11, pp. 1477-1486.

Wilkinson, P. 1995, "A qualitative study to establish the self-perceived needs of family members of patients in a general intensive care unit", Intensive and Critical Care Nursing, vol. 11, pp. 77-86.

Woolley, N. 1990, "Crisis theory: a paradigm of effective intervention with families of critically ill people", Journal of Advanced Nursing, vol. 15, pp. 1402-1408.

Zainal, G. \& Scholes, J. 1997, Adapting to crisis: The relatives' experience of intensive care and the nurses' therapeutic role with the family, University of Brighton, Occasional Paper Series ITU NDU No. 3.

Zapf, D. 2002, "Emotion work and psychological well-being: A r eview of the literature and some conceptual considerations", Human Resource Managment Review, vol. 12, pp. 237-268.

Zigmond, A. S. \& Snaith, R. P. 1983, "The Hospital Anxiety and Depression Scale", Acta Psychiatr, vol. 67, pp. 361-370

Zyzanski, S. J., McWhinney, I. R., Blake, R., \& et al. 1992, "Qualitative research: perspectives on the future," in Doing Qualitative Research, B. F. Crabtree \& W. L. Miller, eds., Sage Publication Ltd., London, pp. 231-248. 
APPENDICES 
Appendix A: LETTERS OF ETHICAL APPROVAL 


\section{NORTH WEST SURREY LOCAL RESEARCH ETHICS COMMITTEE}

The Ridgewood Centre Old Bisley Road

Frimley

Camberley

Surrey

GU16 5QE
Direct Line: 01276605325

Fax: 01276605496

DX: 118800 Frimley 2

Chairs: Dr Day Fernando (Medical Chair)

Mrs Anne Damerell (Lay Chair)

Administrator: Catherine Aikin-Sneath

E-Mail: catherine.aikin-sneath@westsurrey.nhs.uk

10 July 2002

Felicity Hughes

EIHMS student

$5^{\text {th }}$ Floor, Duke of Kent Building

University of Surrey

GUILDFORD

Surrey GU2 7TE

Dear Miss Hughes

$\mathrm{PRO} / 47 / 02$ Please use this reference in all correspondence

The impact of intensive care on relatives

Thank you for submitting the requested amendments, which I reviewed today. I confirm that I consider the amendments to comply with the committee's decision and am therefore able to grant your application full ethical approval via Chair's Actions. Please ensure that approval from the Trust Research \& Development representatives has been obtained prior to commencement of the study.

For your information, the following documentation was reviewed:

- Dual consent form for relative

- Dual consent form for ICU staff

- Information sheets (with amendment to para: "Who has reviewed the study?"

- Information sheet and consent form for patient

Please notify the committee in advance of any significant proposed deviation from the original profocol. Would you also report any unusual or unexpected results, which raise questions about the safety of the research once the study is under way. The committee would be interested in the final results of your study and wish you every success in carrying it out.

The NW Surrey Local Research Ethics Committee operates according to ICH-GCP and applicable laws and regulations.

Yours sincerely

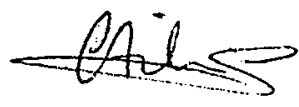

if Dr Dayantha Fernando

JOINT CHAIR 


\section{Unis \\ Advisory Committee ${ }^{\text {on }}$ Ethics}

09 September 2002

Ms F Hughes

EIHMS

Duke of Kent Building

University of Surrey

\section{Dear Ms Hughes}

\section{The impact of intensive care on relatives (ACE/2002/68/EIHMS) - Fast Track}

I am writing to inform you that the Advisory Committee on Ethics has considered the above protocol under its 'Fast Track' procedure and has approved it on the understanding that the Ethical Guidelines for Teaching and Research are observed. For your information, and future reference, these Guidelines can be downloaded from the Committee's website at http://www.surrey.ac.uk/Surrey/ACE/.

This letter of approval relates only to the study specified in your research protocol (ACE/2002/68/EIHMS) - Fast Track. The Committee should be notified of any changes to the proposal, any adverse reactions, and if the study is terminated earlier than expected, with reasons.

Date of approval by the Advisory Committee on Ethics:

09 September 2002

Date of expiry of approval by the Advisory Committee on Ethics: 08 September 2007

Please inform me when the research has been completed.

Yours sincerely

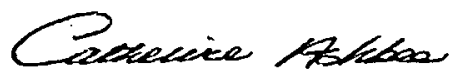

Catherine Ashbee (Mrs)

Secretary, University Advisory Committee on Ethics

Registry

cc: Professor G Ferns, Chairman, ACE 


\section{Brighton and Hove City W/HS}

Primary Care Trust

17 July 2002

Miss F Hughes

EIHMS

$5^{\text {th }}$ Floor

Duke of Kent Building

University of Surrey

GUILDFORD

GU2 7TE

\author{
$6^{\text {th }}$ Floor \\ Vantage Point \\ New England Road \\ BRIGHTON \\ BN1 4GW \\ Direct Line: 01273296437 \\ Direct Fax: 01273295461 \\ kerry.longhurst@bhcpct.nhs.uk
}

Ref: (MS) 02/19

MREC Ref:

From the Mid Sussex LREC

Dear Miss Hughes

\section{Study title: The Impact of Intensive Care on Relatives}

Thank you for your letter and enclosures of 2 July 2002.

I can confirm that the response is satisfactory and I am writing to inform you that Vice-Chairman's

Action has been given to approve this study.

Permission is granted on the understanding that:

i) Any ethical problem arising in the course of the project will be reported to the Committee.

ii) Any change in the protocol or subsequent protocol amendments will be forwarded to the LREC. The principal investigator should see and approve any such changes and this needs to be indicated in the forwarding letter to the Committee.

iii) All serious adverse events must be reported within one week to the Ethics Committee, at the same time indicating that the principal investigator has seen the report and whether or not they feel it poses any new ethical or safety issues.

iv) A brief report will be submitted one year after commencement, thereafter annually, and after completion of the study.

v) Approval is given for research to start within 12 months of the date of application. If the start is delayed beyond this time, applicants are required to consult the Chairman of the Committee.

You are required to obtain separate approval for your project from your host body (e.g. Trust, PCG).

Brighton \& Sussex University Hospitals NHS Trust now operates a research \& development approvals process. If you are an employee of the Trust, or wish to undertake research using either Trust patients or facilities you will need to apply for trust approval before the project can commence. In order to do this you will need to complete and sign a Trust approval project record. To obtain a copy, please contact Scott Harfield, R\&D Manager on 01273696955 ext 7497 or email scott.harfield@bsuh.nhs.uk.

Yours sincerely

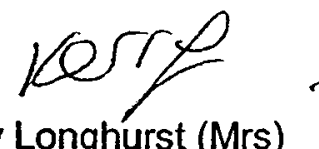

Kerry Longhurst (Mrs)

Research Ethics Committee Administrator 


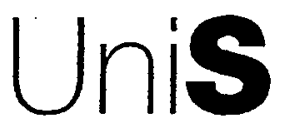

Ethics Committee

24 September 2004

Ms Felicity Hughes

EIHMS

Duke of Kent Building

\section{Dear Ms Hughes}

Relatives experiences of critical care - Phase 2 Information needs of relatives in critical care (EC/2004/90/EIHMS) - FAST TRACK

On behalf of the Ethics Committee, I am pleased to confirm a favourable ethical opinion for the above research on the basis described in the submitted protocol and supporting documentation.

Date of confirmation of ethical opinion: 24 September 2004

The list of documents reviewed and approved by the Committee under its Fast Track procedure is as follows:-

Document Type: Application

Version: 1

Dated: $14 / 09 / 04$

Received: 16/09/04

Document Type: Research Proposal

Version: 1

Dated: 14/09/04

Received: 16/09/04

Document Type: Information Sheet for Relatives

Version: 1

Dated: $14 / 09 / 04$

Received: 16/09/04

Document Type: Information Sheet for Staff

Version: 1

Dated: $14 / 09 / 04$

Received: 16/09/04 
Document Type: Consent Form for Relatives

Version: 1

Dated: 14/09/04

Received: 16/09/04

Document Type: Consent Form for Staff

Version: 1

Dated: 14/09/04

Received: 16/09/04

Document Type: Interview Schedule for Relatives

Version: 1

Dated: 14/09/04

Received: 16/09/04

Document Type: Insurance Proforma

Version: 1

Dated: 14/09/04

Received: $16 / 09 / 04$

Document Type: Letter from Wandsworth LREC

Version: 1

Dated: 14/09/04

Received: 16/09/04

This opinion is given on the understanding that you will comply with the University's Ethical Guidelines for Teaching and Research.

The Committee should be notified of any amendments to the protocol, any adverse reactions suffered by research participants, and if the study is terminated earlier than expected with reasons.

You are asked to note that a further submission to the Ethics Committee will be required in the event that the study is not completed within five years of the above date.

Please inform me when the research has been completed.

Yours sincerely

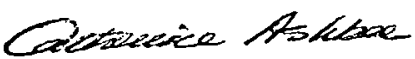

Catherine Ashbee (Mrs)

Secretary, University Ethics Committee

Registry

cc: Professor T Desombre, Chairman, Ethics Committee

Professor K Bryan, Supervisor, EIHMS

Professor I Robbins, Supervisor, EIHMS 
Wandsworth Local Research Ethics Committee

St George's Hospital

Room 27

29 July 2004

1st Floor, Grosvenor Wing

St Georges Healthcare NHS Trust

Blackshaw Road

Tooting, London

SW17 0QT

Ms Felicity Hughes

PhD Student/ Assistant Psychologist

European Institute of Health and Medical

Sciences

5th Floor, Duke of Kent Building

University of Surrey, Guildford

Surrey

GU2 7TE

Dear Ms Hughes,

Full title of study: Relatives' Experiences of Intensive Care

REC reference number: 04/Q0803/25

Protocol number: Protocol Ref N/A

Thank you for your letter of $7^{\text {th }}$ July 2004 , responding to the Committee's request for further information on the above research.

The further information has been considered on behalf of the Committee by the Chairman, Canon lan Ainsworth-Smith and Vice Chair, Dr Christine Heron.

\section{Confirmation of ethical opinion}

On behalf of the Committee, I am pleased to confirm a favourable ethical opinion for the above research on the basis described in the application form, protocol and supporting documentation.

The favourable opinion applies to the following research site:

Site: $\quad$ St. Georges Hospital, London

Principal Investigator: Ms Felicity Hughes

\section{Conditions of approval}

The favourable opinion is given provided that you comply with the conditions set out in the attached document. You are advised to study the conditions carefully.

\section{Approved documents}

The final list of documents reviewed and approved by the Committee is as follows:

Document Type: Application 
Document Type: Investigator CV

Document Type: Covering Letter

Document Type: Interview Schedules/Topic Guides

Document Type: Participant Information Sheet

Document Type: Participant Consent Form

Document Type: Other

\section{Management approval}

The study may not commence until final management approval has been confirmed by the organisation hosting the research.

All researchers and research collaborators who will be participating in the research must obtain management approval from the relevant host organisation before commencing any research procedures. Where a substantive contract is not held with the host organisation, it may be necessary for an honorary contract to be issued before approval for the research can be given.

\section{Notification of other bodies}

We shall notify the research sponsor, St. Georges Hospital, London and the Medicines and Health-Care Products Regulatory Agency that the study has a favourable ethical opinion.

\section{Statement of compliance (from 1 May 2004)}

The Committee is constituted in accordance with the Governance Arrangements for Research Ethics Committees (July 2001) and complies fully with the Standard Operating Procedures for Research Ethics Committees in the UK.

REC reference number: 04/Q0803/25 Please quote this number on all correspondence

Yours sincerely,

$$
\text { Do Ainit. ihe }
$$

\section{Canon lan Ainsworth-Smith Chairman}


Appendix B: EXAMPLE APPLICATION TO ETHICS COMMITTEE 


\section{NHS Research Ethics Committee WHS APPLICATION FORM}

This form should be completed by the chief investigator, after reading the guidance notes. See glossary for clarification of different terms in the application form.

Short title and version number (maximum 70 characters - this will be inserted as header on all forms):

Relatives' Experiences of Intensive Care

Name of NHS research committee to which application for ethical review is being made:

South West London

Project reference number from above REC: $04 / Q 0803 / 25$

Submission Date:

$12 / 05 / 2004$

Keywords: relatives, intensive care, communication, staff perceptions, qualitative, grounded theory

\section{A2. Chief Investigator}

Title: $\quad$ MS

First Name/lnitials: Felicity

Last Name: Hughes

Post:

PhD Student/ Assistant Psychologist

Qualifications: BSc( hons)

Organisation: European Institute of Health and Medical Sciences

Address: $\quad 5$ th Floor, Duke of Kent Building

University of Surrey, Guildford

Surrey

Post Code: GU2 7TE

E-mail: $\quad$ F. Hughesesurrey. ac. uk

Telephone: 01483686737

Fax: $\quad 01483682541$

\section{A3. Proposed Study Dates and Duration}

Start Date: $01 / 07 / 2004$

End Date: $\quad 01 / 01 / 2005$

Duration: Months: $6 \quad$ Years 0 


\section{A4. Primary purpose of the research: (Tick as appropriate)}

Commercial Product development and/or licensing

Publicity funded trail or scientific investigation

घducational Qualification

$\square$ Establishing a Database/Data storage Facility

Other

If other, give details:

\section{A5. Tick the box if you research:}

$\square$ Involves testing a medicinal product

$\square$ Involves testing a medicinal device

$\square$ Involves additional radiation above that for clinical care

$\square$ Involves using stored samples of human biological material (e.g. blood, tissue)

$\square$ Involves taking new samples of human biological material

$\square$ Involves only patient records or data with no other direct patient contact

$\square$ Involves prisoners or others custodial care

$\square$ Involves adults with incapacity

$\square$ Has the primary aim of being educational (e.g. student research a project necessary for a postgraduate degree or diploma, other than MD or PhD)

A6. Do you consider that this research falls within the category where there is no local investigator?
OYes
ONo

If yes please justify: 
A7. What is the principal research question/objective? (Must be in Language comprehensible to a lay person.)

The principal objective is to establish what experience relatives have in the intensive care environment. Specifically, it will look at the nature of communication between relatives and nursing staff, both oral and written. The aim is to ascertain relatives' specific views of the type, amount and nature of information they receive.

\section{A8. What are the secondary research objectives/questions? (If applicable must be in Language} comprehensible to a lay person.)

Secondary objectives are:

1. to develop a protocol of best practice for communication

2. to explore whether the Department of Health guidelines (2000) reflect what staff and relatives feel is needed. .... Cont'd on Additional Pages.

\section{A9. What is the scientific justification for the research? What is the background? Why is this an area of importance? (Must be in Language comprehensible to a lay person.)}

\section{Background}

A review of the literature revealed that the most prominent study in this area to date is still Molter (1979). This is over two decades old. The literature in this area is sparse. Studies that have been carried out have concentrated on relatives needs and most have used the critical care family needs inventory (CCFNI) as an assessment tool. There are few studies using qualitative methods and those that have still focus on relatives' needs rather than overall experience.

The majority of studies about relatives and intensive care have been carried out in the United States. This may bias the findings and affect generalisability, due to. different health care systems between countries. The healthcare system may influence care delivery, so it is important to carry. out similar studies in other countries to distinguish between evidence that is related to intensive care and evidence that is related to the health care system.

Initially I was exploring whether or not the Intensive Care environment was traumatising for relatives. I carried out in depth pilot interviews with relatives and staff to explore relatives' experiences and staff perceptions of those experiences. This did not emerge as the most salient issue. Instead issues emerged surrounding communication processes within intensive care. An analysis of ICU policies concerning relatives and leaflets for relatives was also undertaken. The findings from both phases indicate that the main area of concern is communication, both how and what was communicated. A lot of work has been put into examining communication between staff and patients; little has been done with regard to communication between staff and relatives.

Previous findings from pilot work

Study 1 - Survey of hospital policies concerning relatives

Aim

1. to gauge an overall view of the provisions availabl... Cont'd on Additional Pages. 


\section{A10. Give a brief synopsis/summary of methods and overview of the planned research. This should include a brief description of how prospective research participants and concerned communities (not necessarily geographical) from which they are drawn have been consulted over the design and details of the research.(where appropriate a flow chart or diagram should be submitted separately. It should be clear exactly what should happen to the research participant, how many times and in what order.)}

Study A

Short, structured interviews will be carried out with a sample of up to 20 relatives who currently have a patient in ICU. The sample size will, be determined by theoretical sampling and data saturation. Studies to date have only looked at pre-operative information given to planned admissions (e.g. Derham, 1991, McGaughey and Harrisson, 1994). This study will include planned and unplanned admissions. Relatives will be approached through ICU staff. Interviews should be no longer than 30 minutes (see interview schedule). They will not be tape-recorded. Responses will be recorded on a standardised proforma. The information will be examined in relation to the Department of Health guidelines and be used to help inform the communication protocol.

\section{Study B}

Focus groups will be held with qualified ICU nurses, approx. 3 at a time, to discuss their perceptions of relatives' communication needs and best practice to be. They will be asked to discuss best and worse case admission scenarios; what they should do, what they would do and to what extent they feel they have achieved their aim The information gathered from these focus groups will contribute towards developing a communication protocol for staff to use to deal with relatives. The sample size will be determined by theoretical sampling and data saturation (no new information is being added) (Glaser and Strauss, 1967). It is anticipated that no more than 20 nurses will be recruited. Each focus group will take no longer than 30 minutes. Doctors and other medical staff within the unit will be approached to give their opinion on the protocol. This will ensure that any plan has multidisciplinary input and to establish whether it is workable despite the differences between the role of a doctor and that. of a nurse. Relatives seek different types of information from doctors and nurses, using a multidisciplinary approach aims to produce an outcome that would help both groups.

All participants will be given information sheets and asked to sign a consent form. Participation will be voluntary. Confidentiality, anonymity and the right to withdraw at any time will be assured. Study Abvill be tape recorded and dual consent will be sought. Participants will be asked if they are willing to take part in more focus groups, at a later stage, to give feedback on the leaflet and protocol that have been developed. Using the same participants again will enable them to see tangible results and have feedback from the study.

Continuous revisions will occur throughout the process. Further developments would include an evaluation of the protocol and leaflets using an experimental study within an ICU. 
A11. Would any intervention or procedure, which would normally be considered a part of routine clinical care, be withheld from the research participants?

Give details:

Yes

No

\section{Give details:}

A12. Will the research participants receive any clinical intervention(s) or procedure(s) including taking samples of human biological material over and above that which would normally be considered part of routine clinical care?
Y Yes
ONo

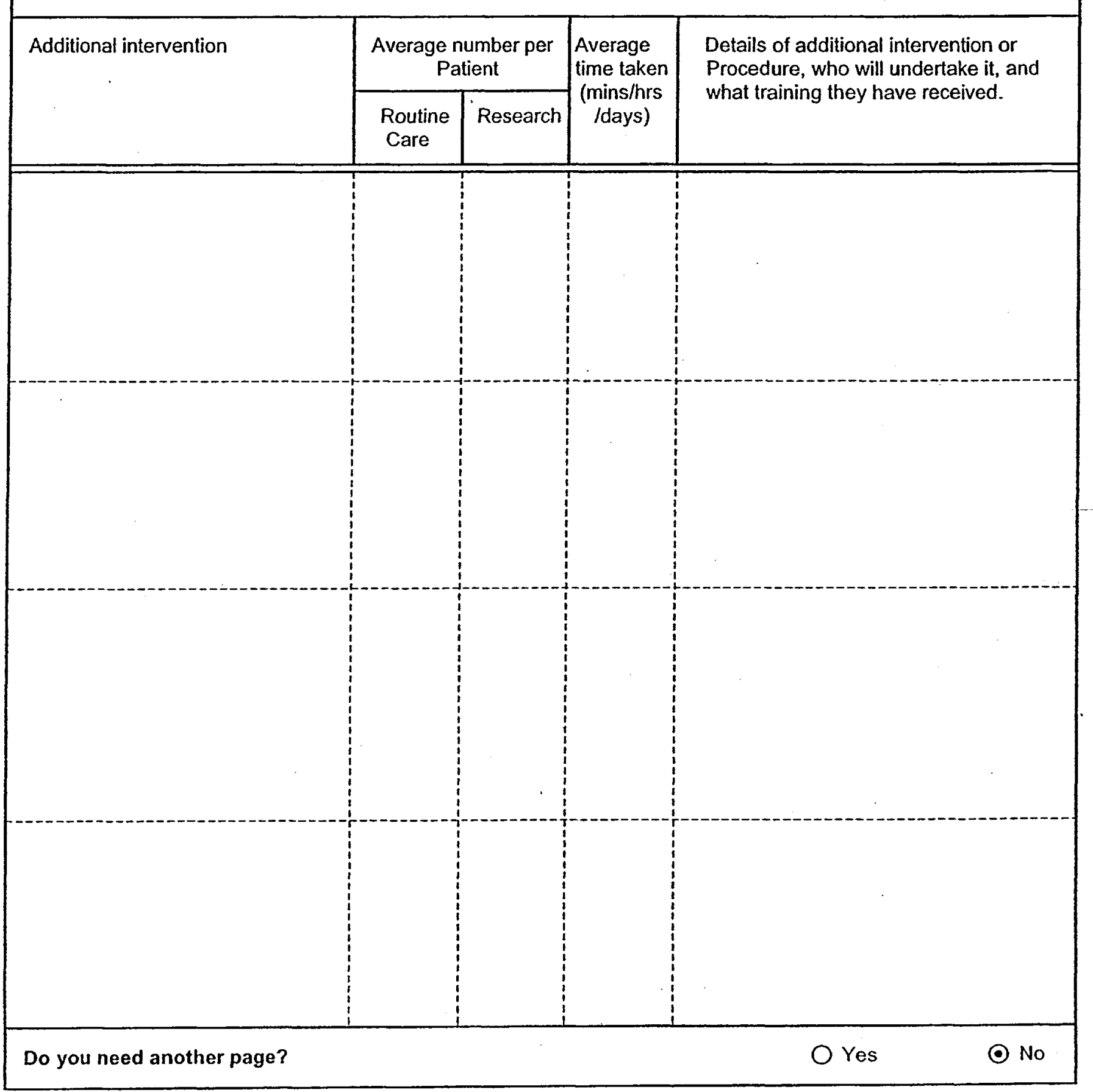


A15. What is the expected total duration of participation in the study for each participant?

Interview for relatives will last approximately 30 mins.

Focus group for staff will last approximately 30 mins.

A16. What are the potential adverse affects, risks or hazards for research participants either from giving or withholding medications, medical devises, jonising radiation, or other inventions(including non clinical)?

$\mathrm{n} / \mathrm{a}$

A17. What is the potential for pain, discomfort, distress, inconvenience or changes to life-style for research participants?

I recognise that it is important to work with the hospital staff, therefore the initial contact with the participants is being made through them. I have been liasing with members of nursing staff about the project. I am not approaching relatives of patients who have died or are dying. The participants always have the option to withdraw at any time, participation is completely voluntary.

If anyone becomes unduly distressed, a member of staff at the Traumatic Stress Service will advise them on best approaches on how to deal with distress or will see them staff at the service are aware of this. Prof Ian Robbins, Head of the Service is also involved in this project.

\section{A18. What is the potential for benefit for research participants?}

Pilot work was carried out in 2 hospitals and feedback from this has been that relatives found it a useful experience to talk to someone unrelated to the situation. Staff said they found it helpful because results may raise issues that they were unaware of. Senior staff were also keen to be able to raise the profile of research within their units.

A19. What is the potential for adverse effects, risks or hazards, pain, discomfort, distress, or inconvenience for researchers themselves? lif any)

I have had training from the Traumatic Stress Service on how to work with distressed people. 
A20. How will potential research participants in the study be(i) identified, (ii) approached and (iii) recruited? Give details for cases and controls separately if appropriate:

Relatives

The participants will be a convenience sample who will be identified by members of staff, who have had a patient on the unit for at least 48 hours. Relatives will be approached initially by members of nursing staff. They will not be approached in the waiting room. They will be informed of the study and if they are willing to participant and give permission I will contact them or they will be given my details and will contact me.

Staff

In conjunction with the unit, I propose to give a series of talks to staff, giving them the opportunity to volunteer.

All participants will be over 18 . All participants will be given information sheets and informed consent will be sought.

A21. Will research participants be recruited via advertisement? OYes - No

Give details: 


\section{A22. What are the principal inclusion criteria? (Please justify)}

Relative is someone who is over 18 years, and considered significant to the patient. The patient must have been in intensive care for at least 48 hours and deemed likely to recover. They will be approached initially by members of nursing staff. This process was negiotiated as part of the pilot work and was very sucessful. Relatives must have a sufficient level of English.

Staff must be qualified ICU staff.

\section{A23. What are the principal exclusion criteria? (Please justify)}

Relatives of patients who have died in Intensive Care or who are so critically ill that the outcome is in doubt.

Relatives who do not speak English.

A24. Will the participants be from any of the following groups? (Tick as appropriate)

$\square$
$\square$
$\square$
$\square$

Children under 16

Adults with learning disabilities

$\square$ Adults who are unconcious or very severly ill

Adults who have a terminal illness

Adults in emergency situations

$\square$ Adults with mental illness(particuarly if detained under Mental Health Legislation)

$\square$ Adults suffering from dementia

$\square$ Prisoners

$\square$ Young Offenders

Adults in scotland who are unable to consent for themselves

Healthy volunteers

Those who couold be considered to have a particuarly dependant relationship with the invetigator, e.g. those in care homes, medical students

[ Other vunerable groups

Justify there inclusion:

Relatives of patients who are in Intensive Care could be distressed by the situation. Although aware of this distress, the pilot study do not find this to be the case. However, this study will bear this in mind and aims to improve how information needs are met. 
A25. Will any research participants be recruited who are involved in existing research or have recently been involved in any research prior to recruitment?

Give details and justify there inclusion:
Yes
No
Not Known

A26. Will informed consent be obtained from the research participants?

OYes $\quad O$ No

Give details of who will take consent and how it will be done. Give details of any particular steps to provide information (in addition to a written information sheet) e.g. videos, interactive material.

If participants are to be recruited from any of the potentially vulnerable groups listed in A24, give details of extra steps taken to assure their protection. Describe the arrangements to be made for obtaining consent from a Jegal representative.

If consent is not to be obtained, please explain why not.

Relatives will initially be approached by members of the nursing staff. Only if they agreed will I then contact them to obtain informed consent. They will not be approached in the waiting room.

A27. Will a signed record of consent be obtained?

Yes

No

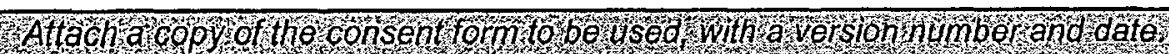

If answer is no, please justify: 
A28. How long will the participant have to decide whether to take part in the research?

$24 / 48$ hours

A29. What arrangements have been made for participants who might not adequately understand verbal explanations or written information given in English? (E.g. translations, use of interpreters etc.)

Although, not automatically excluded, if particpants to not have a sufficient grasp of English they will not be approached.

A30. What arguments are in place to ensure participants receive any information that becomes available during the course of the research that may be relevant to their continued participation? N/a

A31. Does this study have, or require, approval of PIAG (Patient Information Advisory Group) or other bodies with a similar remit? (see Guidance notes)

Give details:
OYes
$\odot$ No

A32. Will the research participant's General Practitioner be informed that they are taking part in the study? OYes

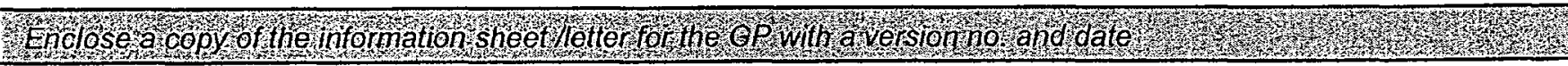

Will permission be sought from the research participants to inform their GP before this is done?

Explain why not:

Y Yes

No

The study involves relatives rather than patients therefore it is not necessary for this study. 
A33. Will individual research participants receive any payments for taking part in this research?

Indicate how much and on what basis this has been decided:

\section{A34. Will individual research participants receive reimbursement of expenses or any other incentives or} benefits for taking part in this research?

Indicate how much and on what basis this has been decided:

OYes $\quad$ No

I will be aiming to see them in conjunction with a normal visit, to minimise disruption on the part of the participant. It is not anticpated that any expenses will occur.

A35. What arrangements have been made to provide indemnity and/or compensation in the event of a claim by, or on behalf of, participants for negligent harm?

Indemnification and/ or compensation will be provided inaccordance with University of Surrey insurance policies.

A36. What arrangements have been made to provide indemnity andlor compensation in the event of a claim by or on behalf of, participants for non-negligent harm?

Indemnification and/ or compensation will be provided inaccordance with University of Surrey insurance policies. 
A37. How is it intended the result of the study will be reported and disseminated? (Tick as appropriate)

x Peer reviewed scientific journals

$x$ Internal report

$x$ Conference presentation

$x$ Other publication

$\square$ Submission to regulatory authorities

$\square$ Access to raw data and right to publish freely by all investigators in study or by Independent Steering Committee on behalf of all investigators

$\square$. Written feedback to research participants

$\square$ Presentation to participants or relative community groups

$\square$ Other/none e.g. Cochrane Review, University Library

If other/none of the above, give details and justify:

Results will also be reported and disseminated in my PhD Thesis.

Based on my pilot work I have had an article accepted for publication and an abstract accepted for an oral presentation at a conference:

Hughes, F., Robbins, I., Bryan, K. (In press) An analy... Cont'd on Additional Pages.

\section{A38. How will the results of research be made available to research participants and communities from} which they are drawn?

The participants will be asked if they would like to be contacted with the results. Reports will be submitted to the units involved. The reports will be subjected to an assessment of readability.

A39. Will the research involve any of the following activities at any stage (including identification of potential research participants)? (Tick as appropriate)

Examination of medical records by those outside the NHS, or within the NHS by those who would not normally have access

Electronic Transfer by magnetic or optical media, email, or computer networks

Sharing of data with other organizations

Export of data outside the European Union

Use of personal addresses, postcodes, faxes, emails or telephone numbers

$\triangle$ Publication of direct quotations from respondents

$\square$ Publication of data that might allow identification of individuals

Use of audio/visual recording devices

Storage of personal data on any of the following:

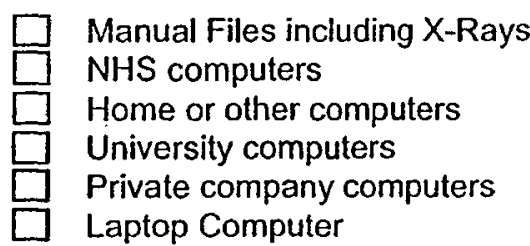

Further details: 
A40. What measures have been put in place to ensure confidentiality of personal data? Give details of weather any encryption or other anonymisation procedures have been used, and at what stage:

No personal data will be recorded or transcribed. All data will be anonymised with a participaint number.

A41. Where will the analysis of the data from the study take place and by whom will it be undertaken? The analysis will be undertaken by myself at the University of Surrey.

A42. Who will have control of, and act as the custodian for, the data generated $b$ the study?

Felicity Hughes - Chief Investigator, under supervision in accordance with the University of Surrey data protection policy.

A43. Who will have access to the data generated by the study?

I will have access to all data. My supervisors will only have access to anonymised data.

A44. For how long will data from the study be stored?

2 Years

0 Months

Give details of where they will be stored who will have access and of the custodial arrangements for the data:

Data will be stored in a locked cabinet that only I will have access to, in accordance with the University of Surrey data protection policy. 


\section{A45. How has the scientific quality of the research been assessed? (Tick as appropriate)}

$x$ Independent external review

$\square$ Review within a company

$\square$ Review within a multi-centre research group

$x$ Internal review (e.g. involving colleagues, academic supervisor)

None external to the investigator

Other, e.g. methodological guidelines

If other, give details:

If you are not in possession of any referees or other scientific critique reports relevant to your proposed study, justify and describe the review process and outcome. If review has been undertaken but not seen by the researcher, Give the details of the body who has undertaken the review:

The study has been subject to external scruntiny as part of the transfer from MPhil to

PhD (October 2003) in accorance with University of surrey requirements.

\section{A46. Has similar research on this topic been done before?}

\section{Why should it be replaced?}

$\odot$ Yes

Exisiting research is dated and has mainly been carried out in the USA. This leads to problems of cross cultural generalisability. There are few qualitative studies, most use quantitative methods such as the Critical Care Family Needs Inventory (CCFNI). There are no studies directly related to the nature of communication process between staff and relatives.

\section{A47. Have all existing sources of evidence, especially systematic reviews, been fully considered?}

Please give details of search strategy used:

A systematic review has been carried out using electronic databases, backward chaining from exisiting articles and extensive discussions with experts in the field. 


\section{A48. What is the primary outcome measure for the study?}

A communication protocol for staff

A49. What are the secondary outcome measures? (If any)

A revised information leaflet for relatives

A50. How many participants will be recruited? How many of these participants will be in a control group? No more than 20 staff and 20 relatives will be recruited. There is no a control group and this is not relevant for the methodology being used (Strauss \& Corbin, 1997). ... Cont'd on Additional Pages.

\section{A51. Has the size of the study been informed by a formal statistical power calculation?}

\section{OYes \\ ONo}

Indicate the basis upon which this was done and giving sufficient information to allow the replication of the calculation:

In consultation with my supervisors, and due to the qualitative nature of the study, it was not considered appropriate to perform a formal statistical power calculation.

A52. Has a statistician given an opinion about the statistical aspects of the research?

Give the name and contact details:

OYes

No

Having sought expert advice on the methodological paradigm being used, it was not deemed appropriate to seek an opinion of a statician.

Give a brief summary of advice offered, and attach a copy of the comments if available: $\mathrm{n} / \mathrm{a}$ 


\section{A53. Describe the statistical methods and/or other relevant methodological approaches (e.g. for qualitative research) to be used in the analysis of the results. Give details of the methods of randomization process to be used if applicable:}

This study with use grounded theory methodology. Grounded theory is particularly suited to the study of local interactions and meanings as related to the social context in which they occur (Gilhooly et al., 2003). It uses the process of constant comparison and theoretical sampling to generate theory. The conceptual categories that are formed from the data generate the theory and this data in turn is used to illustrate the categories. Grounded theory can be applied to any qualitative methods. Most commonly used are interviews.

Constant comparison and theoretical sampling are more than data processing procedures, they build a conceptual and theoretical depth to the analysis. The data are constantly sorted and compared for similarities and differences at all levels e. g. basic data instances, cases, emergent categories and theoretical propositions, throughout the span of the research study to generate conceptual categories.

Theoretical sampling is the active sampling of new data as the analysis proceeds. The analyst decides what data to collect next and where to find it in order to develop the theory. Participants are chosen because they know most about the experiences being explored and will add most to the emerging theory. Additional data are collected to sharpen the definition of the concepts and to define their properties. Negative case analysis may also occur in theoretical sampling, whereby cases are explored that do not appear to fit. This serves to challenge assumptions and strengthen them

Simultaneously collecting (interviews), coding (including writing notes and memos about key concepts and their relationships) and analysing the qualitative data is fundamental to generate the theory. Definite separation of each would hinder the operation (Glaser, Strauss 1967). The traditional distinction disintegrates. Data analysis should commence as soon as there is an adequate amount of material to analyse. The analysis feeds back into sampling new data. Proposing plausible relationships between sets of concepts forms theories.

Collecting, coding and analysing the data was all carried out simultaneously by myself in accordance with grounded theory. As the process of theoretical sampling was carried out the interview guides were revised to help with data interpretation and to identify emerging themes and theoretical saturation.

\section{A54. Where will the research take place? (Tick as appropriate)}

$x$ UK

\section{Other States in the European Union}

Other States in the European Economic Area

Other

\section{Give details:}

St. Georges Hospital, London.

A55. Has this or a similar application been previously rejected by a research ethics committee in the U.K. the European Union or in the European Economic Area?

OYes ONo

Name of research ethics committee or regulatory authority: · South West London REC

Decisions and date taken:

Make revisions \& resubmit 29.03.04

Research ethics committee reference number:

04.0058 
A56. In how many and what type of host organisations (NHS or other) in the UK is it intended the proposed study will take place?

Include the type of organisation by ticking the box and give approximate numbers if known.

Number or organisations

$₫ \quad$ Acule teaching NHS Trusts

1

$\square$ Acule NHS Trusts

$\square$ NHS Community and/or Primary Care Trusts

$\square$ NHS Trusts providing mental healthcare

$\square \quad$ NHS Care trusts

$\square$ Social Care Organisations

$\square$ Prisons

$\square \quad$ Independent hospitals

$\square$ Educational establishment

$\square$ Independent research units

$\square$ Other (Give Details)

\section{A57. What arrangements are in place for monitoring and auditing the conduct of the research?} Regular supervision meetings, regular discussions with unit staff and PhD viva.

\section{Will a data monitoring committee be convened?}

OYes

O No

\section{What are the criteria for electively stopping the trial or other research prematurely?}

Having carried out the pilot study it is considered highly unlikely that this study would need to be stopped prematurely. However, this would occur if it was considered to be posing problems for relatives or staff. 


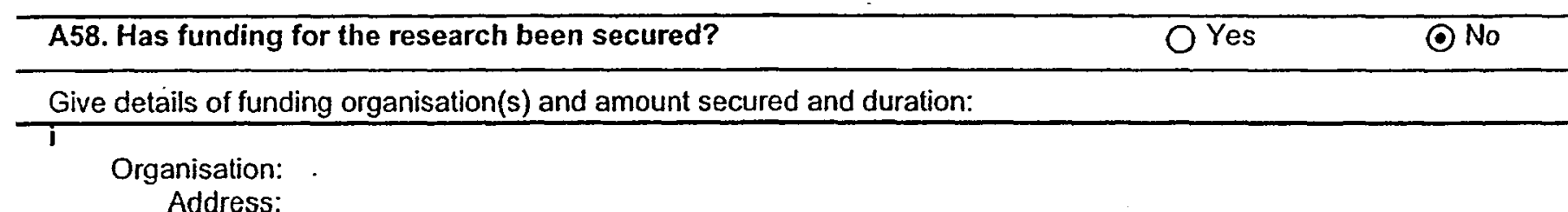

Postcode:

UK Contact:

Telephone:

Email:

Amount: $£$

Duration:

Fax:

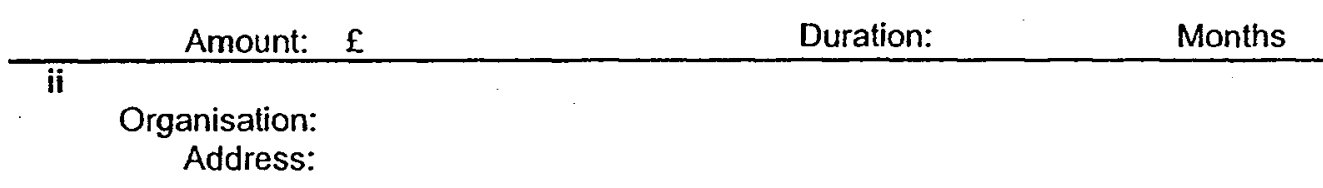

Postcode:

UK Contact:

Telephone:

Fax:

Email:

Amount: $£$ Duration: Months

Postcode:

UK Contact:

Telephone:

Fax:

Email:
Amount: $£$
Duration:
Months

If No, what arrangements are being made to cover any costs of the research? If no external funding is being sought, please say No external funding is being sought.

A59. Has the funder of the research agreed to act as sponsor as set out in the research Governance Framework?

$$
\text { OYes O No }
$$

Not Known

Has the employer of the chief investigator agreed to act as sponsor of the research?
OYes
ONo
Not Known

Give details of the organisation who will act as the sponsor of the research:

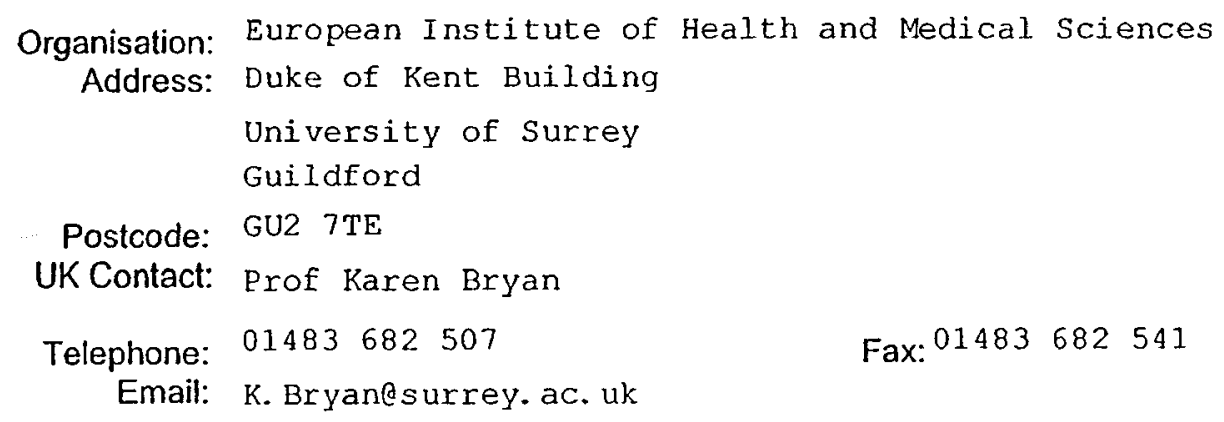


A60. Has any responsibility for the research been delegated to a subcontractor?

Give details including:

OYes $\quad$ No

Name of research contract organisation/site management, and summary of delegated responsibility

A61. Will individual researchers receive any personal payment over and above normal salary for taking part in this research?

Indicate how much and on what basis this has been decided:
OYes
- No

A62. Will individual researchers receive any other benefits or incentives for taking part in this research?

OYes

No

Indicate how much and on what basis this has been decided:

A63. Will the host organiseation or the researchers department(s) or institution(s) receive any payment or benefits in excess of the costs of undertaking the research?

Give details:

OYes $\quad \odot$ No

A64. Does the chief investigator or any other key investigator/collaborator have any direct personal involvement (e.g. financial, share-holding, personal relationship etc.) in the organisation sponsoring or funding the research that may give rise to a possible conflict of interest?

Give details:
OYes
No 
A67. If the research involved a specific intervention (e.g. a drug, medical device, dietary manipulation, lifestyle change etc), what arrangements are being made for continued provision of this for the participant (if appropriate) once the research has finished?

$\mathrm{n} / \mathrm{a}$

\section{PARIA SUMMARY OFETIEALISSUES}

A68. What do you consider to be the main ethical issues or problems may arise with the proposed study, and what steps will be taken to address these?

It is important that the participants are fully informed about the study and that there is no deception. They will be provided with a detailed information sheet and contact details for the researcher should any additional questions arise and adequate time in which to make a decision as to whether they wish to participate. Informed written consent will be sought before participation and a debrief will occur afterwards, together with feedback on the findings if requested.

All participants will be assured of complete confidentiality. Data will be immediately annoymised.

To minimise stress for relatives all initial contact will be made by members of staff. The approach was used for pilot work and proved sucessful. If any participant does become distressed advice can be immediately sought from the Traumatic stress service and if necessary the participant will be seen by a member of their staff.

Every effort will be made to disseminate findings into future practice development. 
A70. Give details of the educational course or degree for which this is being undertaken:

Name and level of course/degree: Research PhD

Name of educational establishment: University of Surrey

Name and contact details of Educational supervisor:
Professor Ian Robbins

Consultant Clinical Psychologist and Head of Traumatic Stress Service, St. George's Hospital, Professor of Mental Health Practice, European Institute of Health and Medical Sciences, University of Surrey. Assistant Director of Psychology for Research and Development, South West London and St. Georges NHS Mental Health Trust.

Contact address:

Traumatic Stress Service... Cont'd on Additional Pages.

\section{A71. Declaration of supervisor}

I have read and approved both the research proposal and this application for ethical review. I undertake to fulfill the responsibilities of a supervisor as set out in the Research Governance Framework for health and social care. I have delegated authority to sign on behalf of my academic institution, that any necessary indemnily or insurance arrangements are in place.

Signature:

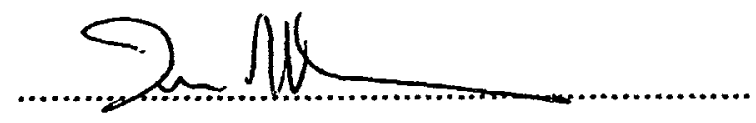

Date: $06 / 05 / 2004$

Print Name: Prof Ian Robbins 


\section{W}

- The information in this form is accurate to the best of my knowledge and belief and I take full responsibility for it.

- I undertake to abide by the ethical principals underlying the Declaration of Helsinki, and good practice Guidelines on the proper conduct of research.

- If the research is approved I undertake to adhere to the study protocol without unagreed deviation and to comply with any conditions set out in the letter sent by the NHS Research Ethics Committee notifying me of this.

- I undertake to inform the NHS Research Ethics committee of any changes in the protocol, and to submit annual reports setting out the progress of the research.

- I am aware of my responsibility to be up to date and comply with the requirements of the law and relevant guidelines relating to security and confidentiality of patent or other personal dala, including the need to register when necessary with the appropriate Data Protection Officer.

- I understand that research records data may be subject to inspection for audit purposes if required in future.

- I understand that personal data about me as a researcher in this application will be held by the Research Ethics Committee and its operational managers, and that this will be managed according to the principals established in the Data Protection Act.

\section{Signature of the Chief Investigator:}

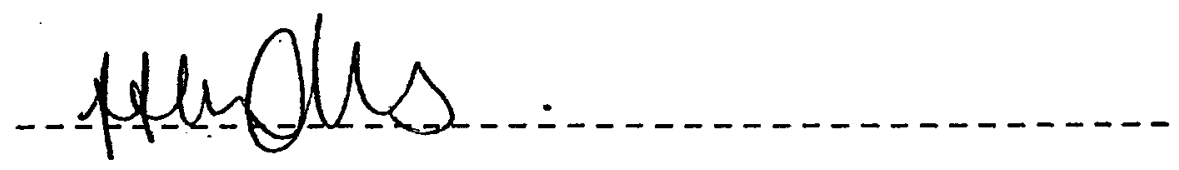

Date:

$06 / 05 / 2004$

Print Name: Felicity Hughes

1.Do you need to add further information about certain questions in part $B$ ?

This question is not applicable for the online version of Corec form. 
C3. Indicate the number of trails/projects within the organisation that the local Principal Investigator has been involved with in the previous 12 months:

How many are still currently (active or recruiting)?

0

Give Details of other members of the local research team responsible to the local Principal Investigator

i

First Name/Initials:

Last Name:

Position:

Qualifications:

Role in team:

ii Title: First Name/Initials:

Last Name:

Position:

Qualifications:

Role in team:

iii Title:

First Name/Initials:

Last Name:

Position:

Qualifications:

Role in team:

C4. Chief Investigator. (Populated from A2)

Title: Ms

First Name/Initials: Felicity

Last Name: Hughes

Post:

PhD Student/ Assistant Psychologist

Qualifications: BSc( hons)

Organisation: European Institute of Health and Medical Sciences

Address:

5 th Floor, Duke of Kent Building

University of Surrey, Guildford

Surrey

Postcode: GU2 $7 \mathrm{TE}$

Email: $\quad$ F. Hughesesurrey. ac. uk

Telephone: 01483686737

Fax: $\quad 01483682541$

C5. Other relevant reference numbers if known (populated from A65):

Applicant's/organisation's own reference number (if available): $\mathrm{n} / \mathrm{a}$

Sponsor's/protocol number: $\quad \mathrm{n} / \mathrm{a}$

Funder's reference number: $\quad \mathrm{n} / \mathrm{a}$

International Standard Randomized Controlled trial Number (ISRCTN): $\mathrm{n} / \mathrm{a}$

European Clinical Trails Database (EUDRACT): $\mathrm{n} / \mathrm{a}$

Project websile: $\mathrm{n} / \mathrm{a}$ 


\section{C6. Give a brief synopsis/summary of methods and overview of the planned research. This should include a brief description of how prospective research participants and concerned communities (not necessarily geographical) from which they are drawn have been consulted over the design and details of the research?(where appropriate a flow chart or diagram should be submitted separately. It should be clear exactly what should happen to the research participant, how many times and in what order.) (Populated from A10)}

Study A

Short, structured interviews will be carried out with a sample of up to 20 relatives who currently have a patient in ICU. The sample size will be determined by theoretical sampling and data saturation. Studies to date have only looked at pre-operative information given to planned admissions (e.g. Derham, 1991, McGaughey and Harrisson, 1994). This study will include planned and unplanned admissions. Relatives will be approached through ICU staff. Interviews should be no longer than 30 minutes (see interview schedule). They will not be tape-recorded. Responses will be recorded on a standardised proforma. The information will be examined in relation to the Department of Health guidelines and be used to help inform the communication protocol.

Study B

Focus groups will be held with qualified ICU nurses, approx. 3 at a time, to discuss their perceptions of relatives' communication needs and best practice to be. They will be asked to discuss best and worse case admission scenarios; what they should do, what they would do and to what extent they feel they have achieved their aim. The information gathered from these focus groups will contribute towards developing a communication protocol for staff to use to deal with relatives. The sample size will be determined by theoretical sampling and data saturation (no new information is being added) (Glaser and Strauss, 1967). It is anticipated that no more than 20 nurses will be recruited. Each focus group will take no longer than 30 minutes. Doctors and other medical staff within the unit will be approached to give their opinion on the protocol. This will ensure that any plan has multidisciplinary input and to establish whether it is workable despite the differences between the role of $a$ doctor and that of a nurse. Relatives seek different types of information from doctors and nurses, using a multidisciplinary approach aims to produce an outcome that would help both groups.

All participants will be given information sheets and asked to sign a consent form Participation will be voluntary. Confidentiality, anonymity and the right to withdraw at any time will be assured. Study A will be tape recorded and dual consent will be sought. Participants will be asked if they are willing to take part in more focus groups, at a later stage, to give feedback on the leaflet and protocol that have been developed. Using the same participants again will enable them to see tangible results and have feedback from the study.

Continuous revisions will occur throughout the process. Further developments would include an evaluation of the protocol and leaflets using an experimental study within an ICU. 
C7. Will the research participants receive any clinical intervention(s) or procedure(s) including taking samples of human biological material over and above that which would normally be considered part of routine clinical care? (populated from A12)

$$
\text { OYes. }
$$

ONo

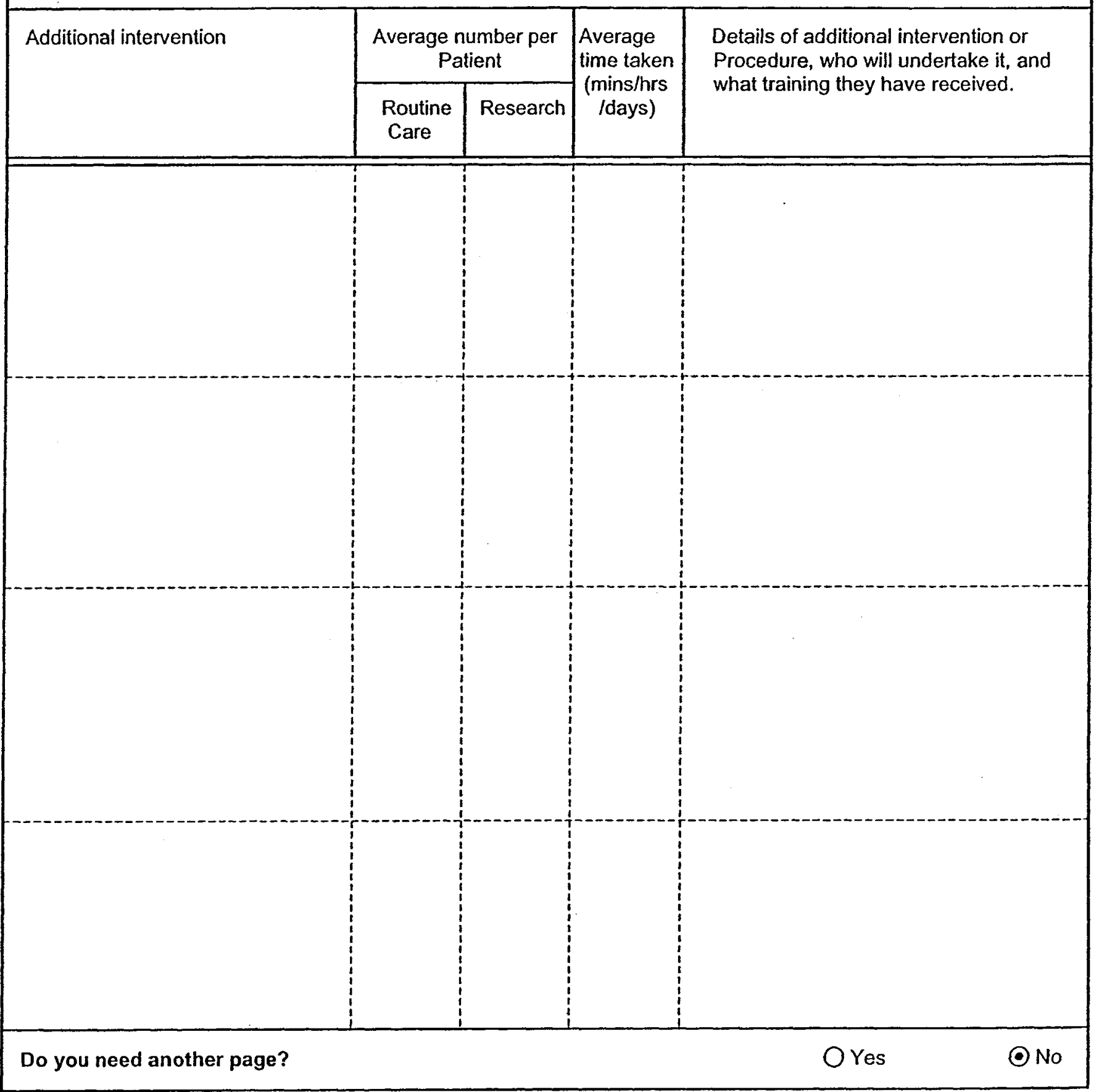


C8. Will the research participant be subject to any non-clinical research-related intervention(s) or procedure(s)? OYes

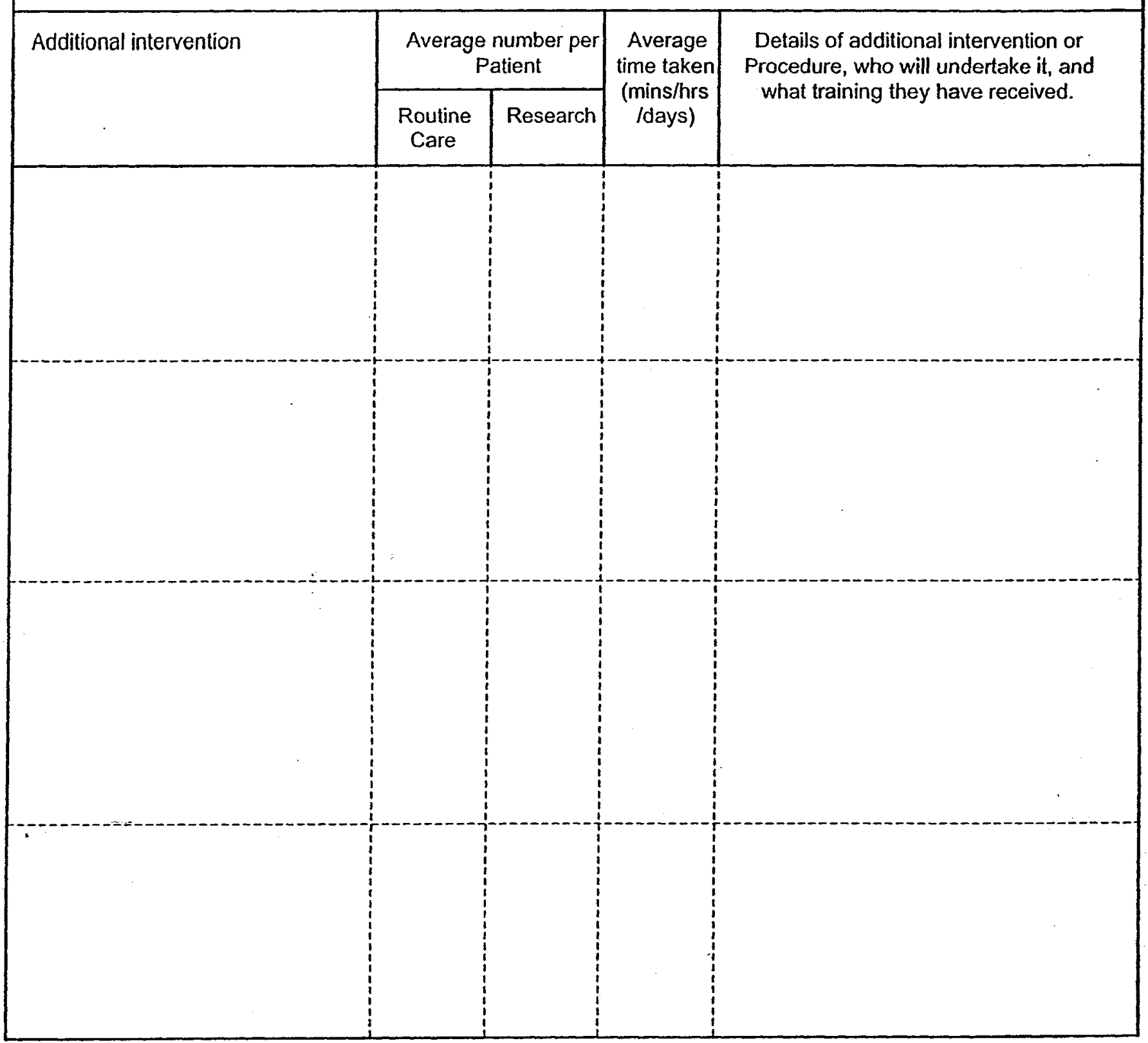

Please give details for other(s): 
C9. Name of NHS or other organisation where the research will take place.

St. Georges Hospital, London

C10. Specify the location(s)/department(s) within the NHS or other organisation where the research will take place.

Intensive Care. Preliminary agreements have been gained from the Cardiothoraic ICU and the General Adult ICU. Contact has been initiated with the Neuro ICU.

C11. how many research participants/samples is it anticipated will be recruited/obtained from this organisation in total?

No more than 20 staff and 20 relatives

C12. Give details of who will be responsible for obtaining informed consent locally, their qualifications and relevant expertise and training in obtaining consent for research purposes:

Following the initial approach by nursing staff, I will be responsible for obtaining informed consent. I have a BSC(hons) in Psyhchology. I have had relevevant training at the University of Surrey and have experience of techniques used through the pilot work already completed.

C13. What local arrangements have been made for participants who might not adequately understand verbal explanations or written information given in English. (E.g. Translation, use of interpreters etc.)

Although, not automatically excluded, if particpants to not have a sufficient grasp of English they will not be approached.

14. What arrangements have been made to inform those responsible for the care of the research participants of their involvement in the research?

Preliminary discussions have taken place on the units and it has been agreed that before the commencement of the study, staff will be made aware and sensitized to the issues through a series of talks. 
C15. Are the facilities and staffing available locally adequate to perform any necessary procedures or interventions required for the study, and to deal with any unforeseen consequences of these? (This should include consideration of procedures and interventions in both control and intervention arms of a study)

Indicate what arrangements are being made to deal with the situation:

○ Yes

No

Immediate advice can be sought from the Traumatic Stress Service, located in the same site. Key links on the units have been involoved in the planning of the study.

C16. Give details of a contact point where participants may obtain further information about the study.

Felicity Hughes

5th Floor, Duke of Kent Building

University of Surrey

Guildford

Please specify the header paper to be used for the patient information sheet.

University of Surrey headed paper

C17. If there is no Principal Investigator at local level, Is there a local individual who is undertaking a task relating to the research?

Give details:
Yes
No
Not Applicable

C18. Do you need to add further information about certain questions in Part C This question is not applicable for the online version of Corec form. 


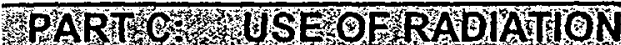

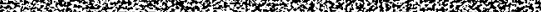

C19. Who is the ARSAC holder taking responsibility for the use of radioanuclide materials?

$\mathrm{n} / \mathrm{a}$

Who will administer the radioanuclide materials?

$\mathrm{n} / \mathrm{a}$

C21. Who is the IRMER practitioner taking local responsibility for the use of diagnostic/therapeutic radiation?

$\mathrm{n} / \mathrm{a}$

C22. Name and signature of the local specialist who has given advice on dose exposure:

Name:

$$
n / a
$$

Role (e.g. RPA or MPE):

Department:

Institution address:

Post Code:

Telephone:

Fax:

I am satisfied that this research proposal complies with the requirements of the IRMER 2000 legislation, and That the radiation doses to research participants will be As Low As Reasonably Practicable (ALARP) According to local best practice.

Signature:

Date: 
- The information in this form is accurate to the best of my knowledge and belief and I take full responsibility for it.

- I undertake to abide by the ethical principals underpinning the declaration of Helsinki, and Good Practice Guidelines on current proper conduct of research?

- If the research is approved I undertake to adhere to the study protocol without agreed deviation and to comply with any conditions set out in the letter sent by the NHS Research Ethics Committee notifying me of this.

- I undertake to inform the NHS Research Ethics Committee of any changes in the protocol, and to submit annual reports setting out the progress of the research.

- I am aware of my responsibility to be up to date and comply with the requirements of the law and relevant guidelines relating to security and confidentiality of patent or other personal data, including the need to register when necessary with the appropriate Data Protection Officer.

- I understand that research records/data may be subject to inspection for audit purposes if required in future.

- I understand that personal data about me as a researcher in this application will be held by the Research Ethics Committee and its operational managers, and that this will be managed according to the principles established in the Data Protection Act.

Signature of local principal Investigator * Signature:

Date:

$06 / 05 / 2004$

Print Name: Felicity Hughes

*The Chief Investigator should sign where there is no local Principal Investigator for the research locality 7f

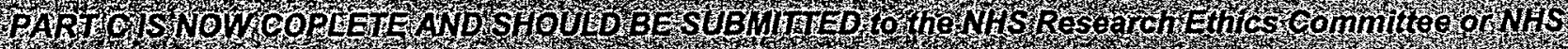
Hog 


\section{Additional Information}

to Part's A, B \& C 
Answer from Question A8 Cont'd...

Reference: Department of Health (2000) Comprehensive Critical Care: A review of adult critical care services. The Stationary office, London.

\author{
Answer from Question A9 Cont'd... \\ e across general ICUs in England for relatives at the current time by conducting an \\ analysis of hospital policies concerning relatives and information available to \\ relatives.
}

2. to see the extent to which the recommendations outlined in the Comprehensive Critical Care review have been implemented nationally, 3 years since the review was published.

The sample analysed consisted of information from 118 hospitals; a 56\% response rate. Eighty four percent (99) of the units had a leaflet of some description for relatives. However these varied greatly in standard and amount of information provided. Only $9 \%$ (10) of the units had some form of official policy concerning relatives. of those who responded 15\% (18) of the units had neither a policy nor leaflet for relatives and $7 \%$ (8) of the units had both a policy and leaflet for relatives.

The information in the leaflets was divided into categories. These categories were determined by the government guidelines and by the information that was received. The frequency of information that fell into these categories was recorded. Categories included, information about equipment, staff, visiting hours, religious support and non-religious support among other things.

These figures can only give general ideas of the type of information relatives are provided with. They do not indicate how well the issues are dealt with. A clear example is the information on equipment, some leaflets go into great depth about all the different machines and their uses other merely comment that

"...During your relatives stay in the Critical Care Department they may be attached to a variety of machines/monitors..." (visitors information booklet for the critical care department)

Each type would have been classed under the category of equipment. Similarly under the staff category, some leaflets named the staff on the ward and their role, other merely commented that you might find nurses, doctors and physiotherapists.

Sixteen per cent of the units that do not have any form of leaflet are not complying with the DoH recommendations. There is huge variation nationally, over the amount and quality of information relatives have access to and receive. There is no consistent nation-wide policy of how staff should deal with relatives when they have a patient in ICU. This implies that the care a relative receives when in an intensive care environment is 'pot luck' depending on which hospital their patient is admitted to. obviously, information that is or is not available to relatives has no reflection on the care that a patient receives while in ICU.

Study 2 - Relatives' experiences of intensive care

Aim

1. To identify themes and concepts in order to try to understand relatives overall experiences of the intensive care environment

2. To identify themes and concepts in order to try to understand how staff perceived these experiences.

Qualitative semi-structured interviews were carried out with ICU nurses and 2 samples of relatives; relatives of brain injured patients in ICU and relatives of patients in a general ICU. Staff generally perceive quite accurately what relatives experience when 
they have family member in an intensive care unit. The traumatic element for the relative seems to be what put the patient in intensive care as oppose to intensive care itself.

There is general dissatisfaction across both samples with the process of receiving information. The amount of information that relatives require is very individual. As the nurses are aware, some people want to know everything and others do not. Being able to assess this is an ongoing nursing challenge. Staff are aware of the importance of giving information, yet some still acknowledge that they do not volunteer information. Relatives seemed to gain the information they require in a very haphazard fashion. One relative had to ask if her husband was in a coma.

The inconsistent nature in which relatives receive information is reflected in the national inconsistency concerning policies and leaflets relating to them It could be said that while staff do not always know specific needs for a particular relative, they have a good perception of the big picture. Molter (1979) identified that the 2 most important needs of relatives were 'to feel there was hope' and 'to feel that hospital personnel cared about the patient.' In the above study it also seemed important for relatives to feel that the hospital personnel care about them. Much of what staff said outlined what was best practice, it was apparent that this did not always occur, predominantly due to stretched resources and inexperienced staff. The latter of which would improve with time. It is important to try and develop consistency in care of relatives so that the experience they have is not a chance occurrence.

\section{References}

Department of Health 2000, Comprehensive Critical Care: A review of adult critical care services. The Stationary office, London.

Glaser, B. G. \& Strauss, A. I. 1967, The Discovery of Grounded Theory: strategies for qualitative research Aldine de Gruyter, New York.

Molter, N. C. 1979, "The needs of relatives of crtically ill patients: A descriptive study.", Heart and Lung, vol. 8, no. 2, pp. 332-339.

Answer from Question $A 37$ Cont' d...

sis of information available to relatives in intensive care. Intensive and critical Care Nursing.

British Psychological Society, Division of Health Psychology Annual Conference. Sept 2004. Oral Presentation: Relatives Experiences of Intensive Care.

Answer from Question A50 Cont'd...

Strauss, A. I. \& Corbin, J. (1997) Grounded theory in practice. London: Sage.

Answer from Question A70 Cont'd...

Clare House

St. George's Hospital

Blackshaw Road

Tooting

SWI 7 OQT

Telephone: $020 \quad 87250355$

Fax: $020 \quad 87250354$

E-Mail: Ian. Robbinseswlstg-tr. nhs. uk

Professor Karen Bryan 
Professor of Clinical Practice, European Institute of Health and Medical Sciences, University of Surrey. Consultant Speech and Language Therapist, Broadmoor Hospital

Contact Address:

European Institute of Health and Medical Sciences

Duke of Kent Building

University of Surrey

Guildford

Surrey

GU2 7 TE

Telephone: 01483682507

Eax: 01483682541

E-Mail: K. Bryanesurrey. ac. uk

Answer from Question C16 Cont'd...

GU2 $7 \mathrm{TE}$

direct line 01483686737

email F. Hughesesurrey. ac. uk 


\section{isurance Guidelines}

he University holds linsurance policies which cover claims arising from its involvement in clinical trials. The policies are arranged on an nnual basis, and it is only in the special circumstances listed in (b) below that it is necessary to seek prior approval from insurers.

he policies are of two types: liability and no-fault. The liability policies cover the University against legal liability claims (ie where the Iniversity is at fault). The no-fault policy provides compensation to subjects, regardless of liability, in the event of their suffering a significant nd enduring injury (including illness or disease) which, on the balance of probabilities, is attributable to their involvement in the trial.

he following should, however, be noled:

\#) The University's policy does not cover medical and dental practitioners while working in a professional capacity. It is the responsibility of the individuals concerned to obtain medical negligence insurance in their own name through an appropriate medical defence organisation. Any claims alleging negligence against the practitioner should be defended by the appropriate defence organisation but must, also, be brought to the attention of the University's insurers. (Nurses are covered under the University's policies, provided that they are assisting in a trial being undertaken at the University itself, and provided that they only undertake activities which fall within the scope of duties normally expected of nurses. It is assumed that they will have RCN membership).

b) Trials involving the following require special consideration and the insurers' prior approval must be sought:

i. Participants who are pregnant

ii. Participants under the age of 5 years

iii. Conception or contraception

iv. Genetic Engineering Studies not for treatment of disease.

c) The University's insurers expect clinical trials involving drugs to be conducted in accordance with the. Association of the British Pharmaceutical Industry Guidelines. In accordance with these Guidelines, where the trial is sponsored by a pharmaceutical company:

i. The company should issue the ABPI standard form of indemnity. This will indemnify the University against all claims and proceedings brought by subjects arising out of their participation in the trial except where the claim arises from the negligence or wrongful act of the University, or its failure to conduct the study in accordance with the protocol. Such claims would be covered by the University's liability policies.

ii. Responsibility for paying compensation should be clarified and reflected in the contractual documentation. This should include an undertaking that compensation will be offered by the company on a no-fault basis (ie. regardless of liability) and paid in the event of a subject suffering a significant and enduring injury (including illness or disease) which, on the balance of probabilities, is attributable directly to their involvement in the trial. The amount of any compensation should be appropriate to the nature, severity and persistence of the injury. The offer of compensation must not prevent the subject from alternatively pursuing a claim on the basis of either negligence or strict liability.

'd) It has been agreed with the University's insurers that the subject's GP will be contacted regarding their suitability for inclusion in a drug trial, particularly where there is no sponsoring pharmaceutical company and for any other clinical trials where the subject's health and medical record is relevant.

(e) For insurance purposes, it is essential that students acting as investigators are supervised by an employee of the University.

March 2004 
Appendix C: PHASE I INFORMATION SHEET AND CONSENT FORM FOR RELATIVES 


\section{University of Surrey}

Guildford

Surrey GU2 $7 \times \mathrm{H}$, UK

Telephone

+44 (0) 1483300800

Facsimile

$+44(0) 1483300803$

uww.surrey.ac.uk

\section{$\underline{\text { Information sheet }}$}

\section{Relatives' Experiences of Intensive care}

\section{European Institute of Health and Medical Sciences}

University Campus

Duke of Kent Building

Stag Hill

Guildford

Surrey GU2 7TE

Telephone

+44 (0) 1483686700

Facsimile

+44 (0)1483686701

You are being invited to take part in a research study. Before you decide it is important for you to understand why the research is being done and what it will involve. Please take time to read the following information carefully and discuss it with others if you wish. Please ask if there is anything that is not clear or if you would like more information. Take time to decide whether or not you wish to take part. I am an independent researcher and should you choose to take part your responses will have no bearing on the care your family member receives.

Thank you for reading this.

\section{Introduction}

This is an exploratory pilot study, with the aim of investigating what experiences people go through when they have a family member in an intensive care unit. In addition it will look at the perception of the nurses and medical staff as to what they think relatives experiences are.

The aim of this study is to establish whether further research is required into the impact of intensive care on relatives and to feed the findings back into the system so as to try to improve the experiences of others that face a similar situation to you.

\section{What will I have to do?}

All you have to do is answer some questions about your experiences of intensive care. Your experiences are important. There are no right or wrong answers. The interview will last approximately 30 minutes at a time and place convenient to you. The interview will be tape-recorded.

After the interview, you will also be asked to fill out a short standardised questionnaire, used to measure the effect of a specific life event on individuals. It will only take approximately 10 minutes to complete. 


\section{Do I have to take part?}

It is up to you to decide whether or not to take part. It is completely voluntary. If you do decide to take part you will be given this information sheet to keep and be asked to sign a consent form. If you decide to take part you are still free to withdraw at any time and without giving a reason. A decision to withdraw at any time, or a decision not to take part, will not affect the standard of care your family member receives.

\section{Will my taking part in the study be kept confidential?}

All information that is collected about you during the course of the research will be kept strictly confidential. The tapes will be transcribed by myself only and any information by which you could be identified will be removed from the transcripts. Once transcribed the tapes will be destroyed.

\section{What do I do now?}

If you are interested in taking part please fill in the bottom section of this information sheet and put it in the collection box in the relatives' room or give it to a member of staff. I will then be in contact with you. Alternatively, you can contact me directly on the details below.

\section{Who has reviewed the study?}

This research project has been considered by our local Research Ethics Committee.

\section{Contact for further information.}

If you require any further information on the above research project, or have any questions please do not hesitate to contact me.

Felicity Hughes

European Institute of Health and Medical Sciences

$5^{\text {th }}$ Floor

Duke of Kent Building

University of Surrey

Guildford GU2 7TE

Direct Line Telephone: $01483686737 \quad$ E-Mail: F.Hughes@surrey.ac.uk

I am interested in taking part in the study on the impact of intensive care on relatives.

Name

Contact telephone number.

Date. 


\section{University of Surrey}

Guildford

Surrey GU2 7XH, UK

Telephone

+44 (0) 1483300800

Facsimile

+44 (0) 1483300803

Centre Number::

www.surtey.ac.uk
Study Number:

Patient Identification Number for this trial:

\section{CONSENT FORM}

Title of Project: Impact of intensive care on relatives
European Institute of Health and Medical Sciences

University Campus

Duke of Kent Building

Stag Hill

Guildford

Surrey GU2 7TE

Telephone

$+44(0) 1483686700$

Facsimile

+44.(0)1483686701

Name of Researcher: Felicity Hughes

Please initial box

1. I confirm that I have read and understand the information sheet dated

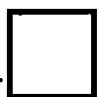
for the above study and have had the opportunity to ask questions.

2. I understand that my participation is voluntary and that I am free to withdraw at any time, without giving any reason, without the medical care of my relative or legal rights being affected.

3. I agree to take part in the above study.

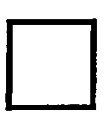

Signature

4. Having completed the interview, I confirm that I agree for the information given to be used in the above study.

Name

Date

Signature

Researcher

Date

Signature 
Appendix D: PHASE I INFORMATION SHEET AND CONSENT FORM FOR STAFF 


\section{University of Surrey}

Guildford

Surrey GU2 7XH, UK

Telephone

$+44(0) 1483300800$

Facsimile

+44 (0) 1483300803

www.surrey.ac.uk

\section{Information sheet}

Relatives' Experiences of Intensive care
European Institute of Health and Medical Sciences

University Campus

Duke of Kent Building

Stag Hill

Guildford

Surrey GU2 7TE

Telephone

+44 (0) 1483686700

Facsimile

+44 (0)1483686701

You are being invited to take part in a research study. Before you decide it is important for you to understand why the research is being done and what it will involve. Please take time to read the following information carefully and discuss it with others if you wish. Please ask if there is anything that is not clear or if you would like more information. Take time to decide whether or not you wish to take part. I am an independent researcher and should you choose to take part your responses will not be disclosed to the hospital.

Thank you for reading this.

\section{Introduction}

This is an exploratory pilot study, with the aim of investigating what experiences people go through when they have a family member in an intensive care unit. In addition it will look at the perception of the nurses and medical staff as to what they think relatives experiences are.

The aim of this study is to establish whether further research is required into the impact of intensive care on relatives and to feed the findings back into the system so as to try to improve the experiences of relatives.

\section{What will I have to do?}

All you have to do is answer some questions about your opinions of what relatives' experience when they are faced with an intensive care environment. The interview aims to find out your opinions. There are no right or wrong answers. The interview will last approximately 30 minutes at a time and place convenient to you. The interview will be tape-recorded. 


\section{Do I have to take part?}

It is up to you to decide whether or not to take part. It is completely voluntary. If you do decide to take part you will be given this information sheet to keep and be asked to sign a consent form. If you decide to take part you are still free to withdraw at any time and without giving a reason.

\section{Will my taking part in the study be kept confidential?}

All information that is collected about you during the course of the research will be kept strictly confidential. The tapes will be transcribed by myself only and any information by which you could be identified will be removed from the transcripts. Once transcribed the tapes will be destroyed.

\section{What do I do now?}

If you are interested in taking part please sign point number 3 on the attached consent form, fill out your contact details and place it in the collection box in the staff room. I will then contact you to arrange a convenient time to conduct the interview. Alternatively you can contact me directly on the details below.

\section{Who has reviewed the study?}

This research project has been considered by our local Research Ethics Committee.

\section{Contact for further information.}

If you require any further information on the above research project, or have any questions please do not hesitate to contact me.

Felicity Hughes

European Institute of Health and Medical Sciences

$5^{\text {th }}$ Floor

Duke of Kent Building

University of Surrey

Guildford

GU2 7TE

Direct Line Telephone: 01483686737

E-mail: F.Hughes@surrey.ac.uk 
University of Surrey

Guildford

Surrey GU2 $7 \times \mathrm{H}$, UK

Telephone

+44 (0)1483300800

Facsimile

$+44(0) 1483300803$

Centre Number::

Study Number:

\section{CONSENT FORM}

Title of Project: Impact of intensive care on relatives

Name of Researcher: Felicity Hughes www.surrey.ac.uk
European

Institute of

Health and

Medical

Sciences

University Campus

Duke of Kent Building

Stag Hill

Guildford

Surrey GU2 7TE

Telephone

$+44(0) 1483686700$

Facsimile

+44 (0)1483686701

\section{Please initial box}

1. I confirm that I have read and understand the information sheet dated

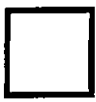
for the above study and have had the opportunity to ask questions.

2. I understand that my participation is voluntary and that I am free to withdraw at any time, without giving any reason, without my legal rights being affected.

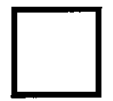

3. I agree to take part in the above study.

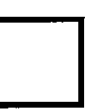

$\overline{\text { Name }} \overline{\text { Date }} \overline{\text { Signature }}$

4. Having completed the interview, I confirm that I agree for the information given to be used in the above study.

Name

Date

Signature

Contact details

Researcher

Date

Signature 
Appendix E: PHASE I INITIAL INTERVIEW SCHEDULES 


\title{
Impact of intensive care on relatives
}

\author{
Relatives interview schedule
}

\section{Introduction}

In my information sheet I said that I am interested in relatives experiences of intensive care. As part of my pilot study, I would like to ask you a few questions about your experiences of the intensive care environment. I am interested in your opinions there are no right or wrong answers. The aim is to feed my findings back into the system so as to try to improve the experiences of others that face a similar situation to you.

I have my questions written down here so I don't forget them. With your permission I will record the interview, so that I can not misinterpret what you say by taking incomplete notes. I will transcribe the interview myself and everything you say will be strictly confidential. Any information that could identify you will be removed from the transcripts.

Is there anything you would like to ask?

\section{Demographic information}

What is your relationship with the patient?

Why is your family member in intensive care?

Have you had any previous hospital experience?

If so, what?

\section{Key questions}

Can you tell me about your experiences of having a relative in intensive care?

Please can you describe the aspects that you found hardest to cope with?

How did you deal with them?

Please can you describe the aspects that you found made the experience easier to cope with?

How were you kept informed about the progress of your relative's condition?

Where/ who/ what was your main avenue for support?

How did that help you?

Are there any other factors away from the hospital that have made things easier or more difficult to deal with? If so, what are they and what effect did they have? 
And finally can I ask:

How old are you?

Are you employed in a health-related profession?

If no, what is your occupation?

Is there anything else that you would like to say that I haven't asked you?

Is there anything you want to ask me?

Explain what going to do with the data analysis and what is going to happen to the results etc

Thank you very much for your time. 


\section{Impact of intensive care on relatives}

\section{ICU staff interview schedule}

\section{Introduction}

In my information sheet I said that I am interested in relatives experiences of intensive care. As part of my pilot study, I would like to ask you a few questions about what you think a relative's experiences are when a patient is in intensive care. I am interested in your opinions, there are no right or wrong answers. The aim of this study is to establish whether further research is required into the impact of intensive care on relatives.

I have my questions written down here so I don't forget them. With your permission I will record the interview, so that I can not misinterpret what you say by taking incomplete notes. I will transcribe the interview myself and everything you say will be strictly confidential. Any information that could identify you will be removed from the transcripts.

Is there anything you would like to ask?

\section{Key questions}

Can you tell me about what you think a person's experiences are when they have a relative in intensive care?

What do you think affects how relatives cope?

Examples?

Please can you describe the things that you think makes the experience easier for relatives to deal with?

Please can you describe the things that you think makes the experience harder for relatives to deal with?

What sort of things are the relatives told?

Please can you describe what you are able to do to support the relatives?

To what extent do you feel satisfied with what you can offer relatives?

If you feel the need for improvement, how do you feel this could be achieved?

Is there anything else that you would like to say that I haven't asked you?

Is there anything you want to ask me?

Explain what going to do with the data analysis and what is going to happen to the results etc

Thank you very much for your time. 
Appendix F: SAMPLE PRESENTATION MADE TO STAFF 


\section{UniS}

European Institute of Health and Medical Sciences

The Impact of Intensive Care on Relatives

Felicity Hughes

PhD Studentship

University of Surrey

\section{UniS}

\section{Supervisors}

Karen Bryan

Professor of Clinical Practice, Consultant Speech and Language Therapist, Broadmoor Hospital

Ian Robbins

Professor of Mental Health Practice, Head of Traumatic Stress Service, St. George's Hospital. 


\section{UniS}

\section{Today}

- Approval

- Aims

- Reasons why

- Methods

- Ethical Issues

- Recruitment

- What to do with the findings

\section{UniS}

\section{Approval}

- Mid Sussex Local Research Ethics Committee

- North West Surrey Local Research Ethics Committee

- Brighton and Sussex University Hospitals NHS Trust Research and Development Committee

- St. Peters and Ashford Hospitals NHS Trust Research and Development Committee

- University Ethics Committee 


\section{UniS}

\section{Aims}

- to find out how relatives experience the intensive-care environment

- to gain the view of staff on what relatives experience

\section{UniS}

\section{Why?}

- There is very little research in this area.

- Most studies are USA based where ICU admission policies differ.

- ICU's can cause a crisis situation for the family.

- Memory function influences psychological trauma. 


\section{UniS}

\section{Why else?}

- Most studies based on the Critical Care Family Needs Inventory (CCFNI) (Molter, 1979).

- The main UK study by Dyer (1991) looks at whether staff could assess if relatives needs were being met, not what these needs were.

- Needs do not necessarily equate to experience.

\section{UniS}

\section{What I want to do}

Relatives

- Relatives approached once they have been told that the family member will be discharged.

- Tear off slip on information sheet.

- Individual, tape recorded, semi structured interviews.

- Impact of Events scale (Weiss \& Marmar, 1997). 


\section{Unis}

\section{What if a relative gets upset?}

If a relative gets distressed during the interview I will:

Refer them to a named member of staff on the unit.

Refer them to charities such as Headway.

Refer them to my supervisor - Professor Ian Robbins, Consultant Clinical Psychologist, Traumatic Stress Service, St. George's hospital.

\section{UniS}

\section{Whatelse I want to do}

Staff

- Information sheets in staff room.

- Consent form attached.

- Return to an allocated box.

- Individual, tape recorded, semi structured interviews. 


\section{How many people?}

A sample size of 10 relatives and 10 staff, split between Hurstwood Park Neurological Centre, Haywards Heath and St Peters Hospital, Chertsey.

\section{UniS}

\section{Ethics}

- All the interviews are confidential.

- I will transcribe all the tapes.

- I will not transcribe any information that will identify people. 


\section{UniS}

\section{Recruitment}

\section{Relatives}

- Over 18 years.

- Considered significant to the patient.

- Patient must have been in Intensive Care for 48 hours or more.

- The patient must have been told that they are being discharged.

\section{Staff}

- Anyone who wants to take part.

\section{UniS}

\section{Findings}

When the pilot study is completed I can:

- write a report for the unit?

- present results? 


\section{Summary}

- Pilot study looking at relatives experience of Intensive care

- Area with very little research

- Interviewing staff and relatives

- Use findings to inform main study

\section{UniS}

\section{References}

Molter N.C. (1979) The needs of relatives of crtically ill patients: A descriptive study. Heart and Lung 8, 332-339.

Weiss, D, S. \& Marmar, C. R. (1997). The Impact of Events Scale - Revised. In J. Wilson \& T. Keane (eds). Assessing Psychological Trauma and PTSD. New York: Guildford.

Glaser, B. \& Strauss, A. L. (1967). The Discovery of Grounded Theory: Strategies for qualitative Research. Aldine de Gruyter: New York.

Dyer LD. (1991) Meeting the needs of visitors - a practical approach. Intensive Care Nursing 7, 135-147. 
Appendix G: PHASE II LETTER SENT TO CRITICAL CARE UNITS 
«Hospital»

«Unit»

«Address 1»

«Address_2》

«Address_3»

«Postcode»

$13^{\text {th }}$ March 2003

\section{University \\ of Surrey}

Guildford

Surrey GU2 7XH, UK

Telephone

$+44(0) 1483300800$

Facsimile

+44 (0)1483300803

www.surrey.ac.uk

Dear Sir/ Madam

I am currently studying for a $\mathrm{PhD}$ at the University of Surrey.

My study is examining relatives' experiences of intensive care. I have carried out a series of pilot interviews with staff and relatives to try and gauge what relatives' experience when they are in an intensive care environment and how staff perceive this experience.

For the next stage, I am proposing to carry out an analysis of hospital policies relating to relatives. I would greatly appreciate it if you could send me copies of:

- Any leaflets available to relatives about your ITU service

- Any other policy documents concerning relatives in your ITU service

A self-addressed envelope is enclosed for your convenience. If you do not have any leaflets or policies concerning relatives, please could you sign and return the attached sheet.

Any information provided is entirely confidential and anonymous. It will be used to inform the final stage of my project. I would be happy to inform you of any results if requested.

Approval from the University Ethics Committee has been gained for the study. Please do not hesitate to contact me if you have any questions. Thank you for your co-operation.

Yours faithfully

Felicity Hughes (Miss)

F.Hughes@surrey.ac.uk

Direct Line - 01483686737 
University

of Surrey

Guildford

Surrey GU2 7 XH, UK

Telephone

$+44(0) 1483300800$

Facsimile

+44 (0) 1483300803

uww.surrey.ac.uk
European Institute of Health and

Medical Sciences

University Campus

Duke of Kent Building

Stag Hill

Guildford

Surrey GU2 7TE

Telephone

+44 (0) 1483686700

Facsimile

+44 (0)1483686701

$<<$ Name of unit $>>>$

(Please tick where appropriate)

We have no leaflets available to relatives about our ITU service

We have no policy documents concerning relatives in our ITU service.

Signed

Name (please print)

Job Title 
Appendix H: PHASE III INFORMATION SHEE'T AND CONSENT FORM FOR RELATIVES 


\section{South West London and St. George's WHS Mental Health NHS Trust}

\section{Information sheet}

\section{Relatives Experiences of Critical Care}

\section{Phase 2- Information needs of relatives within Critical Care}

You are being invited to take part in a research study. Before you decide it is important for you to understand why the research is being done and what it will involve. Please take time to read the following information carefully and discuss it with others if you wish. Please ask if there is anything that is not clear or if you would like more information. Take time to decide whether or not you wish to take part. I am an independent researcher and should you choose to take part your responses will have no bearing on the care your family member receives.

Thank you for reading this.

\section{Introduction}

This study is investigating what experiences people have of the information they receive when they have a family member in a critical care unit.

I realise that this is a difficult time for you. The aim of this study is to feed the findings back into the system so as to try to improve the experiences of others that face a similar situation to you.

\section{What will I have to do?}

All you have to do is answer some questions about your experiences of communication within a critical care unit. Your experiences are important. There are no right or wrong answers. The interview will last approximately 30 minutes at a time convenient to you.

\section{Do I have to take part?}

It is up to you to decide whether or not to take part. It is completely voluntary. If you do decide to take part you will be given this information sheet to keep and be asked to sign a consent form. If you decide to take part you are still free to withdraw at any time and without giving a reason. A decision to withdraw at any time, or a decision not to take part, will not affect the standard of care your family member receives.

\section{Will my taking part in the study be kept confidential?}

All information that is collected about you during the course of the research will be kept strictly confidential and anonymous. 


\section{What do I do now?}

If you are interested in taking part please fill in the bottom section of this information sheet give it to a member of staff. I will then be in contact with you. Alternatively, you can contact me directly on the details below.

\section{Who has reviewed the study?}

This research project has been considered by the Wandsworth Local Research Ethics Committee.

\section{Contact for further information.}

If you require any further information on the above research project, or have any questions please do not hesitate to contact me.

Felicity Hughes

European Institute of Health and Medical

Sciences

$5^{\text {th }}$ Floor

Duke of Kent Building

University of Surrey

Guildford GU2 7TE

Direct Line Telephone: 0148368673

E-Mail: F.Hughes@surrey.ac.uk

\section{Professor Ian Robbins}

Head of Traumatic Stress Service

Clare House

St. George's Hospital

Blackshaw Road

SW17 0QT

Email: Ian.Robbins@swlstg-tr.nhs.uk

I am interested in taking part in the study on the information needs of relatives within critical care.

Name

Contact telephone number

Date.

Signed 
unit:

\section{CONSENT FORM}

Title of Project: Relatives Experiences of Intensive Care

Name of Researcher: Felicity Hughes

Please initial box

1. I confirm that I have read and understand the information sheet

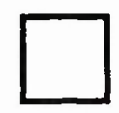
for the above study and have had the opportunity to ask questions.

2. I understand that my participation is voluntary and that I am free to withdraw at any time, without giving any reason, without the medical care of my relative or legal rights being affected.

3. I agree to take part in the above study.
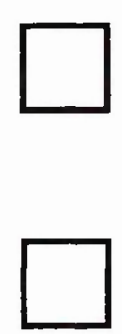

$\begin{array}{ll}\overline{\text { Name }} & \overline{\text { Sate }} \\ \overline{\text { Researcher }} \overline{\text { Date }} & \overline{\text { Signature }}\end{array}$


Appendix I: PHASE III INFORMATION SHEET AND CONSENT FORM FOR STAFF 


\section{South West London and St. George's WHS Mental Health NHS Trust}

\section{Information sheet}

\section{Relatives' experiences of Critical Care}

\section{Phase 2 - Information needs of relatives within Critical Care}

You are being invited to take part in a research study. Before you decide it is important for you to understand why the research is being done and what it will involve. Please take time to read the following information carefully and discuss it with others if you wish. Please ask if there is anything that is not clear or if you would like more information. Take time to decide whether or not you wish to take part. I am an independent researcher and should you choose to take part your responses will not be disclosed to the hospital.

Thank you for reading this.

\section{Introduction}

This study is investigating relatives' experiences and communication practices within critical care settings.

The aim of this study is to establish whether further research is required into the impact of critical care on relatives and to feed the findings back into the system so as to try to improve the experiences of relatives.

\section{What will I have to do?}

All you have to do take part in a focus group with other qualified critical care staff. You will be asked to discuss issues raised by relatives and the concept of best practice. The focus group aims to find out your opinions. There are no right or wrong answers. The focus group will last approximately 30 minutes at a time convenient to you. The focus group will be tape-recorded.

\section{Do I have to take part?}

It is up to you to decide whether or not to take part. It is completely voluntary. If you do decide to take part you will be given this information sheet to keep and be asked to sign a consent form. If you decide to take part you are still free to withdraw at any time and without giving a reason. If you do not wish take part or withdraw this will not affect your employment in any way.

\section{Will my taking part in the study be kept confidential?}

All information that is collected about you during the course of the research will be kept strictly confidential. The tapes will be transcribed by myself only and any 
information by which you could be identified will be removed from the transcripts. Once transcribed the tapes will be destroyed.

\section{What do I do now?}

If you are interested in taking part please sign the first section on the attached consent form, fill out your contact details and place it in the collection box in the staff room. I will then contact you to arrange a convenient time to talk to you. Alternatively you can contact me directly on the details below.

\section{Who has reviewed the study?}

This research project has been considered by Wandsworth Local Research Ethics Committee.

\section{Contact for further information.}

If you require any further information on the above research project, or have any questions please do not hesitate to contact me.

\section{Felicity Hughes}

European Institute of Health and Medical Sciences

$5^{\text {th }}$ Floor

Duke of Kent Building

University of Surrey

Guildford

GU2 7TE

Direct Line Telephone: 01483686737

E-mail: F.Hughes@surrey.ac.uk

Professor Ian Robbins

Head of Traumatic Stress Service

Clare House

St. George's Hospital

Blackshaw Road

SW17 0QT

Email: Ian.Robbins@swlstg-tr.nhs.uk 


\section{South West London and St. George's W/PS Mental Health NHS Trust}

Group no: unit:

\section{CONSENT FORM}

Title of Project: Relatives' Experiences of Intensive Care

Name of Researcher. Felicity Hughes

Please initial box

1. I confirm that I have read and understand the information sheet

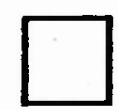
for the above study and have had the opportunity to ask questions.

2. I understand that my participation is voluntary and that I am free to withdraw at any time without giving any reason, without my legal rights being affected.

3. I agree to take part in the above study.

Signature

Name

Date

4. Having completed the focus group, I confirm that $I$ agree for the information given to be used in the above study.

Name

Date

Signature

Researcher

Date

Signature 